
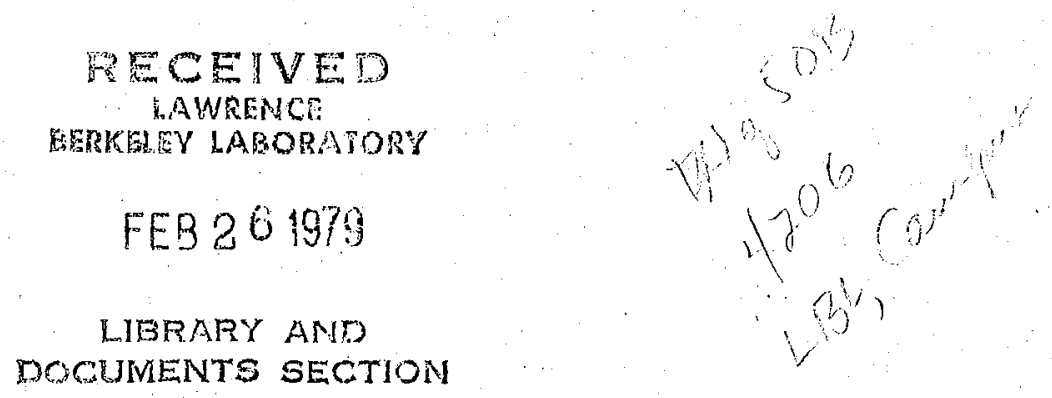

\title{
MASS TRANSFER CONTROLLED REACTIONS IN PACKED BEDS \\ AT LOW REYNOLDS NUMBERS
}

\author{
Peter S. Fedkiw \\ (Ph. D. thesis)
}

December 1978

Prepared for the U. S. Department of Energy under Contract W-7405-ENG-48

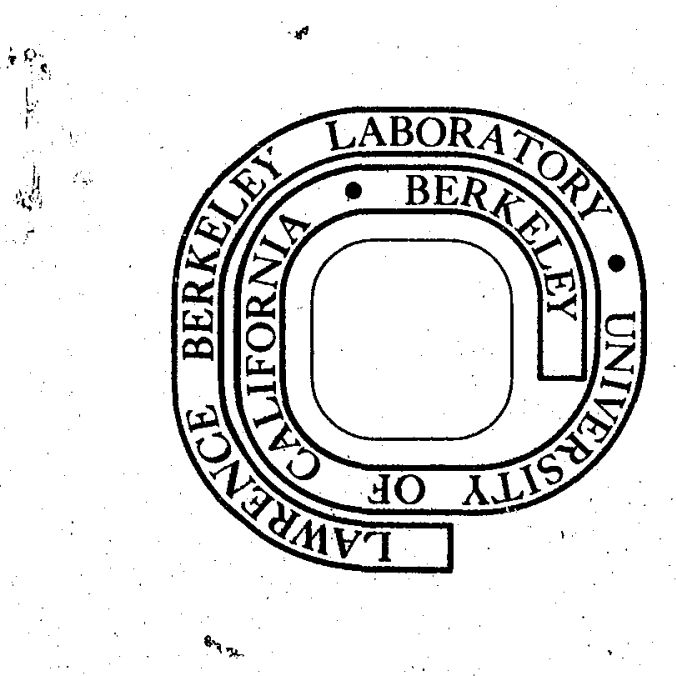

\section{TWO-WEEK LOAN COPY}

This is a Library Círculating Copy which may be borrowed for two weeks. For a personal retention copy, call Tech. Info. Dívision, Ext. 6782 


\section{LEGAL NOTICE}

This repo" vas prepared as an account of " United Sta.

ment of $\mathrm{Er}$.

tractors, st

express or

the accura

ratus, prod

not infring

$y$ the partconranty, ty for ppa'ould 
LBL -8509

Mass Transfer Controlled Reactions in Packed Beds

at Low Reynolds Numbers

Peter S. Fedkiw

Materia1s and Molecular Research Division, Lawrence Berkeley Laboratory and Department of Chemic,al Engineering, University of California, Berkeley, California 94720

December, 1978

Abstract

This dissertation examines the a priori prediction and correlation of mass-transfer rates in transport 1imited, packed-bed reactors at low Reynolds numbers.

The solutions to the governing equations for a flow-through porous electrode reactor indicate that these devices must operate at a low space velocity to suppress a large ohmic potential drop. Packed-bed data for the mass-transfer rate at such low Reynolds numbers have been examined and found to be sparse, especially in liquid systems.

Models which have appeared in the 1iterature to simulate the solidvoid structure in a bed are reviewed. Only within the framework of these geometric models can the fundamental transport equations be solved. In this work the bed was envisioned as an array of sinusoida1 periodically constricted tubes (PCT). No other work exploiting this model for mass-transfer calculations has appeared in the 1iterature. The velocity field in such a tube should be a good approximation to the converging-diverging character of the velocity field in an actual bed. The creeping flow velocity profiles were found by a numerical solution for this geometry. These results were used in the 
convective-diffusion equation to find mass transfer rates at high Péclet number for both deep (Graetz-like) and shallow (Lévêque-1ike) beds. The convective-diffusion equation was also solved for low Péclet numbers in a deep bed. A11 calculations assumed a transport limited condition, wherein the reactant concentration at the tube surface is zero. These calculations were expressed in terms of a mass-transfer coefficient.

Mass-transfer data were experimentally taken in a transport controlled, flow-through porous electrode to test the theoretical calculations and to provide data presently unavailable for deeper beds.

It was found that the sinusoidal PCT model could not fit the data of this work or that available in the 1iterature. However, all data could be adequately described by a model which incorporates a channeling effect. The bed was successfully modeled as an array of dual sized straight tubes. 
for

Sharon

Catherine and Peter 


\section{Acknowledgements}

I'd like to thank Linda McGuire for typing this dissertation, especially for her patience in correcting the revisions.

Special thanks is due to Cecilia Mak for carrying out some of the analytical chemistry. Her "energetic" manner in approaching this task shortened the time required to finish this dissertation.

I find it very difficult to express in a few sentences the gratitude I owe Professor John Newman. It has been a pleasurable and rewarding experience working with Professor Newman. His clear, concise, simplistic approach to defining and resolving a research problem will always be a model for me.

This work was supported by the Divison of Solar, Geothermal, Electric and Storage Systems, Office of the Assistant Secretary for Energy Technology, U.S. Department of Energy under contract No. W-7405-Eng-48. 
Mass Transfer Controlled Reactions in

Packed Beds at Low Reynolds Numbers

\section{Contents}

Abstract

Dedication

Acknowledgements $\quad$ ii

Contents

iii

Chapters

1. Introductory Remarks

2. Limiting-Current Porous Electrodes and MassTransfer Coefficients

3. Mass Transfer at High Péclet Numbers for Creeping Flow in a Packed-Bed Reactor

4. Low Péclet Number Behavior of the Transfer Rate in Packed Beds

5. Numerical Calculations for the Asymptotic, Diffusion Dominated Mass-Transfer Coefficient in Packed-Bed Reactors

6. Entrance Region (Lévêque-1ike) Mass Transfer Coefficients in Packed-Bed Reactors

7. The Measurement of Mass-Transfer Controlled Reaction Rates in an Electrochemical PackedBed Reactor

8. Interpretation of Results 143

Nomenclature

Appendicies

A. $\mathrm{E}^{4}$ Operator in $(n, z)$ Coordinate System 174

B. Listing of Computer Programs 175

C. Solutions of Coupled, Ordinary Differential Equations with Associated Constant Unknowns

D. Data Compilation 
References 


\section{Chapter 1 \\ Introductory Remarks}

This thesis is concerned with the low Reynolds number $(<1)$ behavior of the mass-transfer rate in fixed, packed-bed reactors. Attention is focused on reactions which are controlled by the rate of mass transfer of a reactant from the fluid phase to the packing material. Figure 1.1 is a schematic illustration of the physics in question. One single packing particle is shown in an isolated view from all of its surrounding neighbors. A reaction takes place at the particle surface, or if this is a porous catalyst, within the particle. The transfer of the reactant from the fluid to the particle is the controlling factor in the rate of reaction. It would be advantageous to predict a priori the rate of reaction at the particle. This would involve solving the governing differential equations for fluid flow and mass transfer subject to appropriate boundary conditions. However, this fundamental approach cannot be applied due to the random nature of the particle arrangement. One must resort to models of the geometry of the packing structure in order to solve the governing equations. A major portion of this thesis is concerned with solving the transport equations within the framework of the periodicaliy constricted tube model. This model has recently been proposed in the literature, and no work prior to this effort has exploited this mode1 for packed bed mass-transfer calculations. Of course, no model can be proved successful unless it is compared with experimental data. 

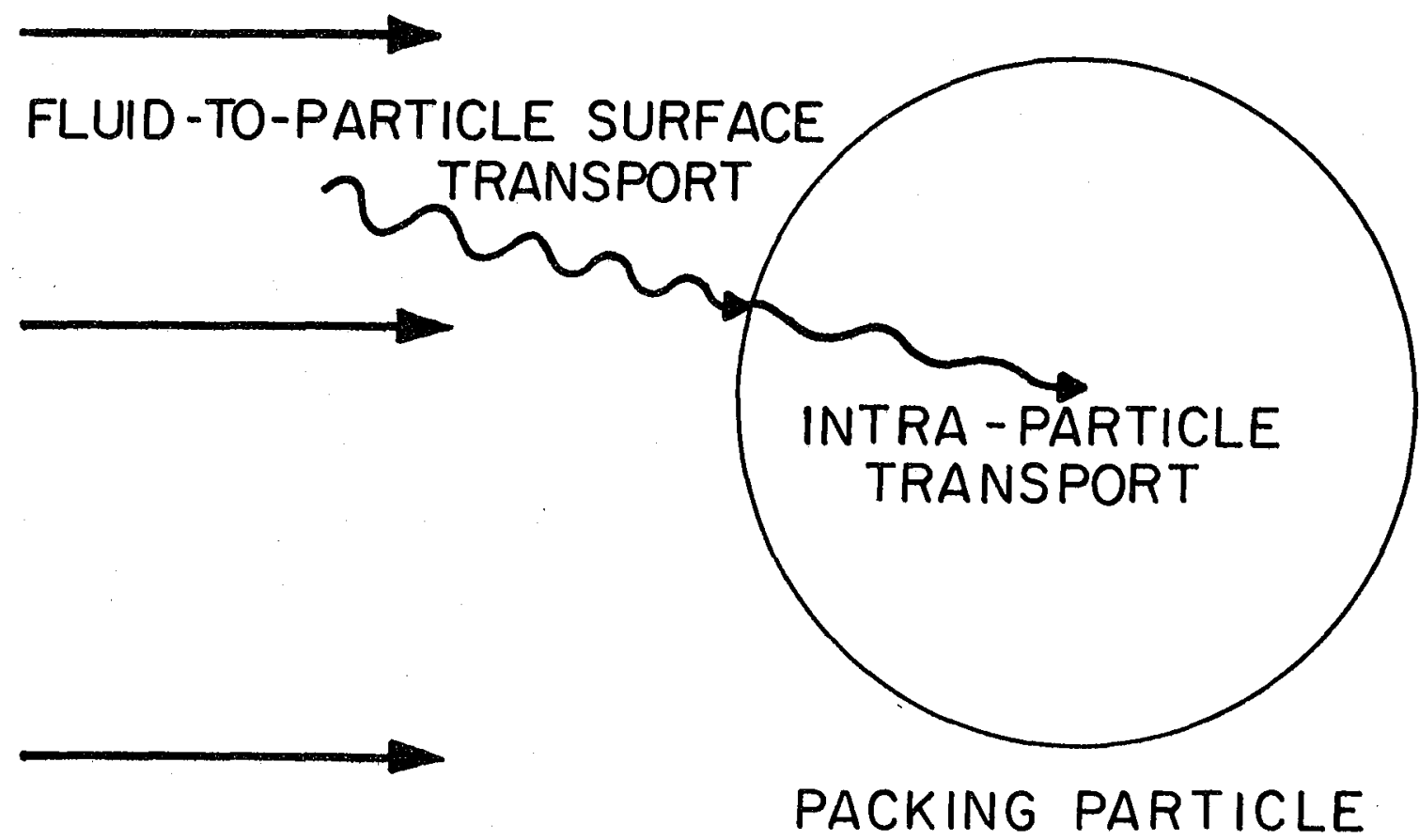

XBL $7811-12879$

Figure 1.1 Isolated packing particle from a two-phase, packedbed reactor. 
As a part of this research effort, an experimental program to measure mass-transfer rates in a transport-controlled porous electrode reactor was carried out. The comparison between data and theory allows one to refine the model when the agreement between the two is not acceptable. This was the situation with the periodically constricted tube model. The data are better fit by a straight tube model which incorporates a channeling effect.

The remainder of this chapter is divided into two sections. The first section introduces and outlines the remaining chapters. The second presents a literature survey of the geometrical models for the void-solid arrangements in a packed bed and discusses the periodically constricted tube model which is used in this work.

\section{Chapter Outlines}

The chemical engineering literature can be gleaned to find reams of work on mass-transfer rates in two-phase packed bed reactors. The results are correlated by a mass-transfer coefficient. The majority of this work, however, is for large Reynolds numbers. There is very little work done at low Reynolds numbers because most unit operations involving packed beds proceed at a high space velocity. Chapter 2 introduces the design equations for a mass-transfer controlled porous electrode in which it is sometimes necessary to operate at a low space velocity. The equations which describe a porous electrode operating at a limiting current (mass-transfer controlled rate of reaction) are solved. These solutions point out the need for more reliable mass-transfer coefficient data in the low Péclet number region 
(i.e., low Reynolds number, high Schmidt number). The need for data taken in deeper beds than are currently available in the Ifterature also becomes apparent.

Chapter 2 is of an introductory nature, but the next four chapters are of a theoretical nature. At the time this thesis was written, all four of these chapters have either appeared in published form (Chapters 3 and 4), or are at the printer's (Chapter 5), or are currently being reviewed for publication (Chapter 6). The titles of the chapters are the same as the published form. These chapters are written in essentially the same format as when they were submitted to the journal for publication. Only minor indexing type changes were made to conform to this thesis. Consequently, there is some unavoidable overlap and duplication.

Chapter 3 (AIChE J., 23, 255, 1977) continues the discussion of the periodically constricted tube (PCT) model. The calculations for the velocity profiles in sinusoidal PCT are presented. With these velocity profiles available, the mass-transfer equations can be solved. The Graetz-1ike eigenvalue problem for the developed mass-transfer rate in a sinusoidal PCT is developed and solved in this chapter and the results applied to the packed bed mass-transfer coefficient.

In the course of examining the literature for mass-transfer coefficient data at low Pêclet numbers, it soon became apparent that there was confusion as to the behavior of the coefficient in this 1imit. Some workers reported that the coefficient approached a constant nonzero value, while others reported it to decrease continually with the Péclet number. 
Chapter 4 (Chem. Eng. Sci., 33, 1043, 1978) explains this discrepancy. The behavior of the mass-transfer coefficient in this limit depends upon its definition. A singular perturbation approach is used to demonstrate this conclusively. The results are generally valid in that no model of the geometry is necessary to draw the conclusions.

Chapter 5 (to appear in Chem. Eng. Sci., 1979) uses the sinusoidal PCT model to add a predictive capability to the analysis of Chapter 4. This chapter presents calculated values for the low Péclet number mass-transfer coefficient for deep beds.

Chapter 6 (submitted to. AIChE J., October, 1978) completes the calculations of asymptotic mass-transfer coefficients in sinusoidal PCT by presenting the Lévêque-1ike values. These mass-transfer coefficients are valid in the entrance region to the mass transfer section at high Péclet numbers. These results are applied to the packed bed.

Chapter 7 discusses the experimental program of this thesis. Transport limited mass-transfer coefficients were measured in a porous flow through electrode constructed of $3.18 \mathrm{~mm}$ spheres.

Chapter 8 suggests empirical formulae to merge the asymptotic mass-transfer coefficients of Chapters 3,5, and 6 to cover the nonasymptotic regions, and attempt to parameter fit the data of Chapter 7 to the PCT model. Careful examination of the data reveals that the PCT model cannot fit these data nor the available literature data satisfactorily. The data suggest that a nonuniform flow distribution is present in the bed and needs to be taken into account. A channeling model consisting of an array of dual sized straight tubes is found to fit the data of this work and 1iterature values. 
Models of the Packing-Void Geometry in a Packed Bed

It would be useful to predict the reaction rate in a packed bed by solving the fundamental three dimensional transport equations subject to appropriate boundary conditions. The effect of flowrate, particle size and shape, and depth of packing on the overall reaction rate in the bed could then be predicted. But even in the simplest case of no kinetic 1imitations with constant concentration along the particle surface, this fundamental approach cannot be used due to the randomness of the particle packing. Alternative routes have been used to approach an understanding of the mass-transfer rates in packed beds. Some of these alternative approaches will now be discussed.

Much experimental work has been done in this field. Most workers have used test systems in which the concentration of the transferring species is constant at the particle surface. This is a we11 characterized system. The results are correlated by a masstransfer coefficient. Some of this work will be discussed in Chapters 2, 4, and 8. This empirical approach is quite useful in correlating the results of this complex physical situation. However, it does have its limitations. Each set of experiments is confined within a certain flowrate and packing depth. The effort of many workers is required to establish a parameter space large enough to formulate a wide ranging correlation. The effect of experimental uncertainty must be considered when examining such data. 
The random nature of the particle pack is the roadblock in applying the fundamental approach. The application of a statistical theory for fluid-particle systems could be used to overcome this difficulty. However, as pointed out by Brenner ( 1 ), "The present status of the subject may be likened to that of nonequilibrium molecular statistical mechanics prior to the advent of the work of Kirkwood." To utilize such an approach for a packing of uniformly sized spheres, the angular distribution of contact points on a central reference sphere is required. With this information, a cell representative of the statistical features of the entire bed can be constructed, and the transport equations solved within this framework. A theory to generate such information does not exist yet. Nayak and Tien ( 2 ) have made a contribution to this effort by developing a statistical theory to predict the local coordination number (total number of contact points, irregardless of orientation) on a particle in a randomly packed bed by maximizing the "entropy" of the configuration. Haughey and Beveridge ( 3 ) have reviewed the statistical structural properties of a packed bed and in another work ( 4) have critically examined the statistical models used to account for the porosity variation about a reference sphere. Many references which pertain to the structural aspect of particle arrangements may be found in these works.

The averaging of the transport equations over a suitable reference volume is another approach used to solve the governing equations. This is the most profitable route to follow in a utilitarian sense. The differential equations thus generated can be solved and the solutions 
are used routinely in design, scaleup, and control. The averaged equations involve phenomenological coefficients such as the dispersion coefficient and the film mass-transfer coefficient. Newman in an appendix of Dunning's thesis ( 5 ) has presented an averaging of the masstransfer equations. These will be discussed in more detail in the next chapter. Slattery $(6,7)$ has discussed the averaging of the fluid motion equations, while Whitaker ( 8 ) and Gray ( 9 ) have discussed the averaging of the convective-diffusion equation. Brenner ( 1 ) has developed a methodology for averaging of the momentum equation taking into account the forces and couples acting on the packing particles.

The final approach examined here is the use of geometrical models for the solid-void arrangement. A major portion of this thesis is devoted to exploiting one of these models, consequently, the following discussion will be in some detail.

Figure 1.2 lists models which are found in the literature. For each of these (except the last) both the fluid motion and mass-transfer equations have been solved.

The bed is envisioned as a spatially periodic replication of the structures shown on this figure. The geometrical parameters of the mode1 geometry are chosen such that the macroscopic parameters of the bed are reproduced (e.g., porosity, average particle size, specific interfacial area, ...)

With these models available, the solutions to the fluid motion and convective diffusion equation become tractable. These solutions 
Figure 1.2 GEOMETRIC MODELS FOR THE SOLID-VOID STRUCTURE IN A PACKED BED

FREE SURFACE-CELL MODEL

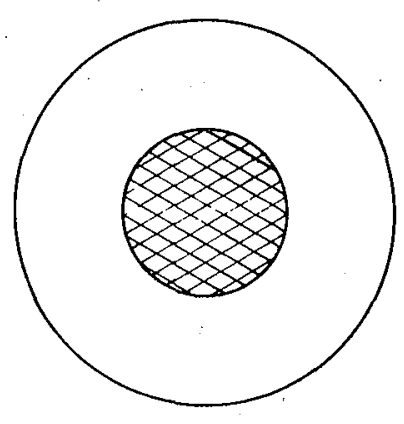

HAPPEL (1958)
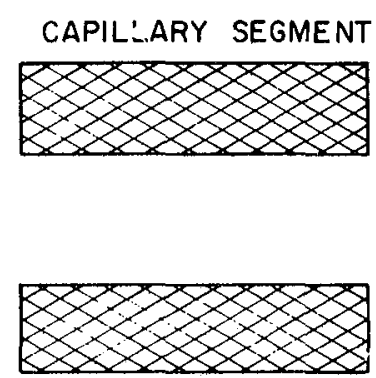

CELL EMBEDDED IN A CONTINUUM

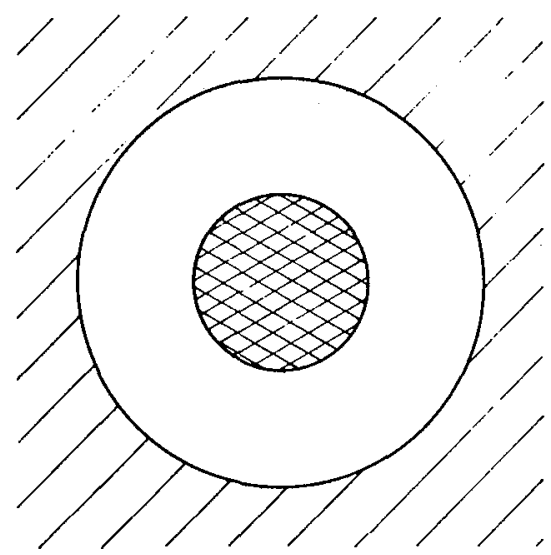

BRINKMAN (1947)

NEALE AND NADER (1974)

SIMPLE CUBIC PACKED SPHERES

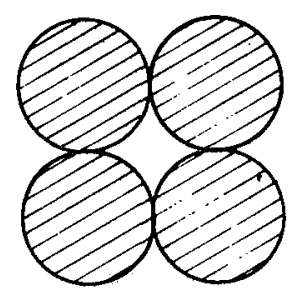

SøRENSEN AND STEWART (1974)

$\theta$

PERIODICALLY CONSTRUCTED TUBE
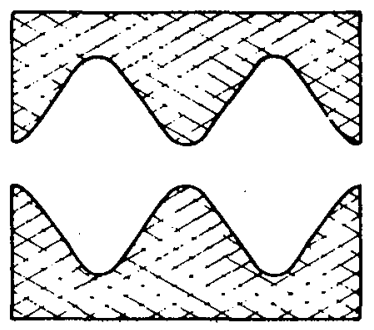

PETERSEN (1958)

PAYATAKES, TIEN, TURIAN (1973)

$X B L 78 \mid-4469$ 
can then be used to predict the phenomenological coefficients of the averaged detailed equations. In this thesis we will be interested in the predictions for the mass-transfer coefficients.

These models may be divided into three classes: those which envision the fluid flow in the bed as equivalent to that past a particle; those which envision the bed flow as equivalent to flow through a conduit (or a network thereof); and, in a class by itself, the simple cubic packed bed of uniform sized spheres considered by Sфrensen and Stewart $(11,12)$.

The calculations of Sørensen and Stewart are a significant piece of work. These authors did not specifically intend their calculations to be a model for a randomly packed bed of spheres, but they do guide one's thinking in approaching this problem. They have numerically solved the convective diffusion equation and fluid motion equations for this geometry and presented results for the mass-transfer coefficient over a large variation of Péclet number and packing depth. Their results will be cited many times in the remainder of this thesis. It has been pointed out $(13,14)$ that the conduit mode1s become a better approximation to the flow paths as the porosity approaches one, whereas the flow past a particle is a better approximation as the porosity approaches zero. In the intermediate range of porosities found in most beds $(0.3<\varepsilon<0.7)$, both models can be applied.

The free surface-cell model was developed by Happel (32). A packing particle is imagined to be isolated from all of its neighbors by a surrounding sphere of fluid. The fluid streams past the outer 
she11 with the superficial bed velocity. The shear at the outer surface is set equal to zero, thus the disturbance caused by the particle is confined to this shell of fluid. Pfeffer and Happe1 (15) have solved the convective-diffusion equation at low Péclet numbers using the creeping flow velocity profile given by Happel (32). However, they used a constant concentration boundary condition at both the particle surface and the outer free surface, which corresponds to a fictitious source of material. This criticism has been raised by Appel (16), Nelson and Galloway (17), and Sфrensen and Stewart (10). Nelson and Galloway (17) attempted to overcome this problem by postulating a surface renewal type boundary condition for the free surface concentration. Criticism of this approach will be postponed until Chapter 5. Pfeffer (18) has solved the high Péclet number convectivediffusion equation for this model with the constant-surface-concentration boundary condition. His results indicate that the mass-transfer coefficient is proportional to the cube root of the Péclet number. E1-Kaissy and Homsy (19) have used a regular perturbation solution to consider inertial effects in this model.

The cell embedded in a continuum model is geometrically similar to Happel's free surface cell. This model was first proposed by Brinkman (20) to predict the permeability of a bed and again by Neal and Nader (13) to predict the effective diffusivity in a packed bed. This model in a sense recognizes that two length scales characterize a packed bed. One is a macroscopic length scale over which significant changes take place in measurable quantities, and the other is a smaller 
length scale of the order of the packing diameter. Two general solutions governing the physical process under consideration are generated, one for the continuum and the other for the ce11. These two solutions are then matched at the she11 boundary. Tardos et al. (21) have used the creeping flow velocity profile given by Neal and Nader (22) to solve the high Péclet number mass-transfer problem for a constant-surface-concentration boundary condition. Their results indicate a cube-root dependence of the mass-transfer rate on the Péclet number. No one has attempted to solve the low Péclet number masstransfer problem with this model. The same criticism which applies to Happel's model also applies here.

The capillary models have been routinely applied in the calculation of permeabilities. Extensive reviews of this subject have been given by Scheidigger (23), Bear (24), and Dullien (14). Surprisingly, there has not been much published work which uses this model for masstransfer rate calculations in a packed bed. The calculations of Sфrensen and Stewart (10) are applicable here. Kataoka et al. (25) have used this conduit model to correlate their mass-transfer data.

In the simplest application, the bed is envisioned as an array of these tubes parallel to the main flow direction and completely passing from one face of the bed to the other. Various amplifications on this theme are possible; e.g., the conduits may be arranged skew to the main flow direction. Consideration of this effect gives rise to the tortuosity coefficient. The bed has also been modeled as a network of channels in which tubes meet at an intersection and branch out in 
a new direction $(26,27)$. These models have not yet been exploited for mass-transfer calculations.

A new conduit model has recently been proposed in the literature. The periodically constricted tube model was suggested by Petersen (28) to explain abnormally high effective diffusivities in catalyst particles. This model was further expanded on by Payatakes et al. $(29,30)$ as a means to predict the permeability of a nonconsolidated bed. These authors have outlined a procedure to determine the model parameters from the macroscopically measured variables of the bed. As originally envisioned by Payatakes et al., the pore space in the bed is generated by the intersection of two parabolic wall channels, thus forming a converging conduit and a diverging conduit each one half period length long and which meet at a cusp. The orientation of this flow channel was parallel to the main flow direction. In a later publication (31), this model was refined to include an angular distribution of these segments about the main flow direction. In this same publication's discussion section it was postulated that some finite number of these segments feed into a central mixing point where the fluid is totally mixed and redistributed to the same number of segments downstream. This is a further refinement of the model leading to lateral mixing via a network.

The converging-diverging character of the flow through these periodically constricted tubes is thought to give a good approximation to the actual velocity profile in the intersticies of the bed. With this approximation to the velocity profile, a more refined 
solution to the convective diffusion equation should be possible. A major portion of this thesis is concerned with solving the transport equations in a sinusoidal periodically constricted tube and applying these results as a model for a packed bed. No other work has been published exploiting this model for mass-transfer calculations. The bed is envisioned as an array of sinusoidal periodically constricted tubes aligned with the main flow direction. The creepingflow motion equation is solved within this geometry, and the solution to the convective-diffusion equation with a constant wall concentration under various limiting conditions of the dimensionless parameters is presented. 
Chapter 2

Limiting Current Porous Electrodes and Mass-Transfer Coefficients

Summary

This chapter presents design equations for a porous electrode operating at the limiting current. The equations involve a dispersion coefficient and a film mass-transfer coefficient, both of which are discussed in some detail. Some available data for effective masstransfer coefficients are presented. The distinction between these two coefficients is pointed out. The solution to the porous electrode equations indicate that additional mass-transfer data are needed for low Péclet numbers and deeper beds than are currently available in the literature.

\section{Porous Electrodes}

The utility of porous electrodes as a unit operations-electrochemical reactor has been discussed by Newman and Tiedemann $(33,34)$. We shall not delve into this area but rather present the differential equations which describe a porous electrode.

The fundamental three dimensional transport equations cannot be solved directly, and hence an alternative approach is used. The equations are averaged to make them more tractable. In the appendix of Dunning's dissertation (5), a discussion of average quantities and a derivation of certain transport equations is discussed. The governing equations for a porous electrode have been given by Newman and Tiedemann (33) in a detailed form in their review article. These equations 
can be reduced to the following form for a steady-state porous electrode with multiple electrode reactions.

Electrode reaction

$$
\sum_{i} s_{i j} M_{i}^{i} \rightarrow n_{j} e^{-}
$$

Mass balance

$$
\varepsilon E \frac{d^{2} c_{i}}{d z^{2}}-v \frac{d c_{i}}{d z}=a k_{f i}\left(c_{i}-c_{i w}\right)
$$

Ohm's Law

$$
i_{2}=-K \nabla \Phi_{2}
$$

Faraday's Law

$$
k_{f i}\left(c_{i}-c_{i w}\right)=\sum_{j} \frac{s_{i j}}{n_{j} F} f_{j}
$$

Kinetic rate expression

$$
\nabla \cdot i_{2}=a \sum_{j} f_{j}\left(\eta, c_{i w}\right)
$$

Equation (1) is an abstract representation for the electrode reaction; e.g., in $\mathrm{Cu}^{++}$deposition, $\mathrm{Cu}^{++}+2 \mathrm{e}^{-}+\mathrm{Cu}$. Equation (2) is a mass balance on component $i$ and includes a dispersive flux where $E$ is the dispersion coefficient. The concentrations are an average over the volume of the solution in the pores, and $z$ is the streamwise coordinate in the bed. The term $a k_{f i}\left(c_{i}-c_{i w}\right)$ represents the rate of consumption of reactant $i$ per unit volume of the bed, where $k_{f i}$ is the film mass-transfer coefficient for reactant $i$, and $c_{i w}$ is 
the concentration at the packing surface. Ohm's Law follows from the assumption of a we11-supported electrolyte. The effective conductivity $\kappa$ is taken as $\varepsilon^{1.5} \kappa_{0}$ (33) where $\kappa_{0}$ is the free stream value. The current density $i_{2}$ in the solution is referred to the entire cross section of the electrode, and $\Phi_{2}$ is the volume-averaged potential of the solution phase. Faraday's Law relates the wall flux of reactant $i$ to the appropriately summed rates of its consumption in electrochemical reaction $j$. The kinetic expression $f_{j}$ relates the divergence of $i_{2}$ to the appropriate driving forces for the $j^{\text {th }}$ reaction which are the local overpotential and the local wall concentrations. A Butler-Volmer type of kinetic equation is an appropriate form for this function.

This set of equations has been numerically solved subject to boundary conditions by Trainham and Newman (35) for the special case of dilute metal ion recovery. The evolution of $\mathrm{H}_{2}$ was incorporated as a secondary reaction only in the kinetic expression. Alkire and Gould (36) have also solved these equations for multiple metal ion recovery. Alkire and Gracon (37) simulated a single electrode reaction by solving these equations. Further reviews may be found in Newman and Tiedemann (34).

It is useful to examine the solution to these equations when the mass transfer of the reactant from the solution to the packing controls the rate of reaction. Such a situation becomes physically realizable if the exchange current density for the reaction is very high. In the recovery of electropositive heavy metal ions this is 
usually a good approximation. In this case, the kinetic expression is no longer necessary, and the system of equations one needs to examine is given by 2,3 , and 4 with $c_{i w} \ll c_{i}$.

Newman and Tiedemann (34) have solved these equations for a single electrode reaction. The solution depends upon the boundary conditions imposed. The following solution satisfies the Wehner-Wilhelm (38) boundary conditions for the concentration and assumes that the packing matrix is at a uniform potential. The counterelectrode is placed upstream of the bed. This is the configuration shown at the top of Figure 2.1. (A discussion of the various configurations and the effect on electrode performance has been given by Trainham and Newman, 39.)

$$
\begin{gathered}
\frac{c_{L}}{c_{F}}=\theta_{L}=\frac{e^{-y / B}+\frac{D^{\prime}}{B^{2}} e^{B y / D^{\prime}} \exp \left[-\alpha L\left(\frac{1}{B}+\frac{B}{D^{\prime}}\right)\right]}{B+\frac{D^{\prime}}{B^{2}}(1-B) \exp \left[-\alpha L\left(\frac{1}{B}+\frac{B}{D^{\prime}}\right)\right]} \\
\Delta \Phi_{2}=\frac{n F V c_{F}}{s K} \frac{v}{a k_{f}}\left[B^{2} \theta_{L} e^{\alpha L / B}-\frac{D^{\prime}}{B}-\left(\alpha L+1+D^{\prime}\right) \theta_{L}\right]
\end{gathered}
$$

where

$$
y=\frac{a k_{f}}{v} z, D^{\prime}=\frac{\varepsilon a k_{f}}{v^{2}} E, B=\frac{1+\sqrt{1+4 D^{\prime}}}{2}
$$

Equation 6 gives the concentration of the reactant leaving the bed, and equation 7 expresses the solution ohmic potential drop across the electrode. When the axial dispersive flux is unimportant, $D^{\prime}$ becomes sma11, and equations 6 and 7 reduce to the expressions first given by Bennion and Newman (40). 

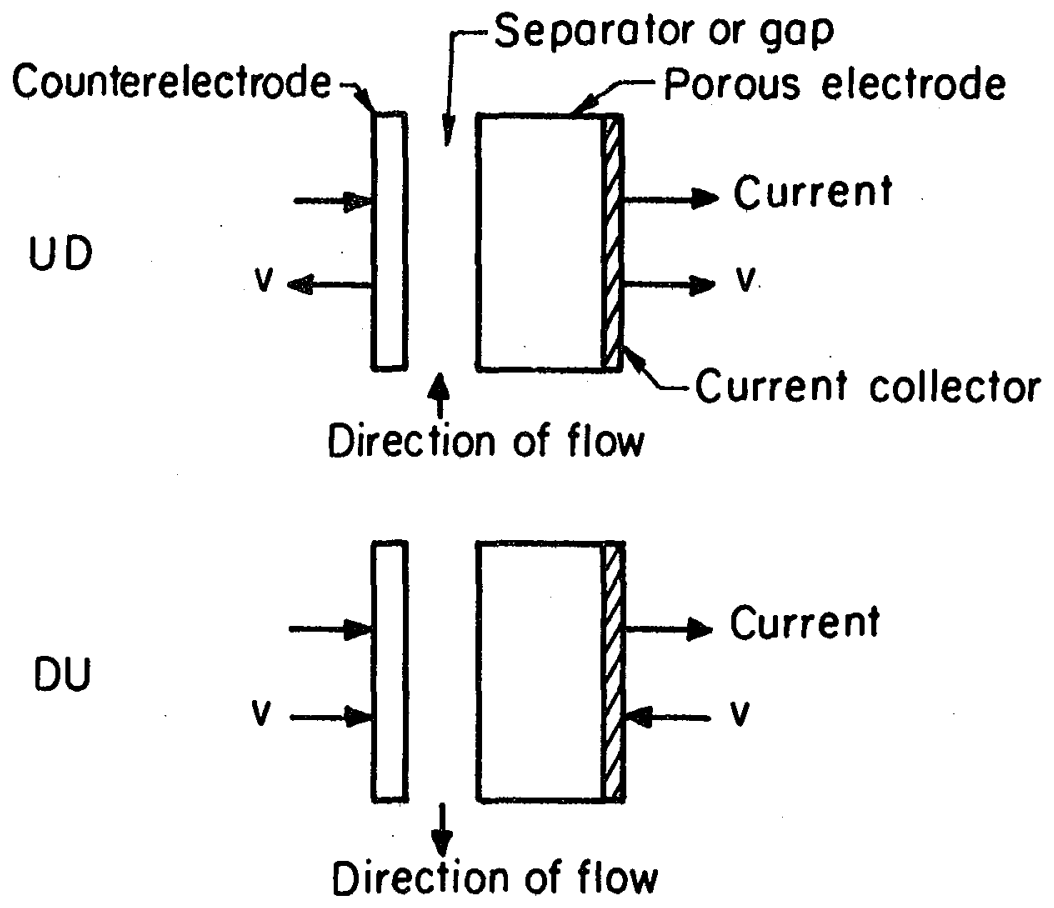

UU

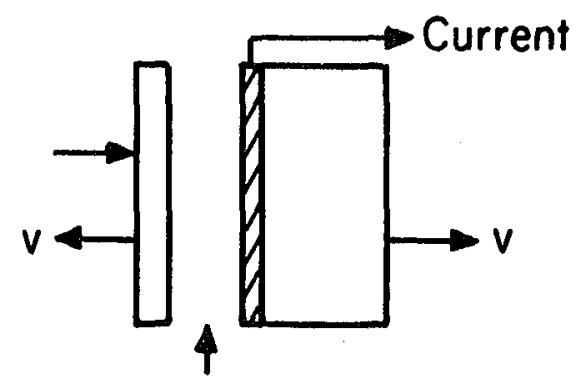

\section{Direction of flow}

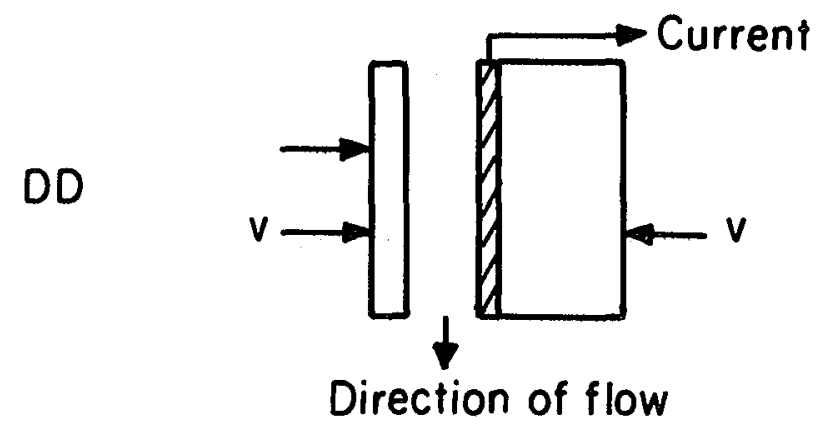

XBL774-5249

Figure 2.1 Various configurations of counterelectrode placement and current collector placement relative to the direction of the fluid flow. (Taken from reference 39.) 
The behavior of these solutions is dependent upon the phenomenological coefficients $k_{f}$ and $E$. Before we discuss these solutions, a discussion on these two coefficients is in order.

\section{Dispersion Coefficient}

The necessity of a dispersion coefficient is a direct consequence of our ignorance of the detailed velocity and concentration fields in the intersticies of the packing. It is not a fundamental quantity but rather a derived quantity which is generated by averaging the detailed three dimensional transport equations. It consequently also depends upon the boundary conditions at the particle surfaces for the fields in question. This fact has not always been recognized in the literature.

Sankarasubramanian and Gill (41) have solved the convectivediffusion equation in a pipe with first-order kinetics at the reactive wa11. They have demonstrated that the dispersion coefficient generated by this solution when the kinetic rate constant is large is an order of magnitude smaller than the dispersion coefficient calculated by Taylor (42) in the absence of a reactive wall.

All of the dispersion coefficient data and correlations known to this author are taken from beds with nonreactive particles. This should be kept in mind, because one is forced to utilize these results for want of something more appropriate.

Sherwood et a1. (43) have reviewed dispersion coefficient data in their text. They present in graphical form a compilation of a large number of workers' results for the dispersion coefficient as a function of Reynolds and Schmidt number. 
Some authors have assumed that the dispersion coefficient is simply the sum of a molecular diffusion term and a turbulent eddy term. One may then write

$$
\frac{E}{v d_{p}}=\frac{D_{o}}{\tau v d_{p}}+\frac{1}{2 \varepsilon} .
$$

Other, more sophisticated models for the dispersion coefficient have been presented in the literature. The model developed by Gunn (44) and by Miyauchi and Kirkuchi (45) is of most interest here. These workers have realized that the dispersion coefficient in creeping flow cannot reach the turbulent eddy value given by the second term of equation 9. The details of their theory are unimportant. It is impressive that Miyauchi and Kirkuchi were able to fit dispersion coefficients in creeping flow over 10 orders of magnitude of the Péclet number with their calculations. The correlation is in a convenient equation form given below.

$$
\frac{E}{D_{0}}=\frac{1}{\tau}+\frac{v}{a D_{0}} \frac{4}{0.17 x}\left[1-\frac{1}{2 x}\left(1-e^{-2 x}\right)\right]
$$

where

$$
\mathrm{x}= \begin{cases}10.666 /\left(\mathrm{v} / \mathrm{a} D_{\mathrm{o}}\right)^{6 / 7} & \left(\mathrm{v} / \mathrm{a} D_{\mathrm{o}}\right)<15 \\ 8.731 /\left(\mathrm{v} / \mathrm{a} D_{\mathrm{o}}\right)^{2 / 3} & \left(\mathrm{v} / \mathrm{a} D_{\mathrm{o}}\right)>15\end{cases}
$$

Figure 2.2 is a plot of the dispersive Péclet number ( $/ \mathrm{aE})$ as a function of the molecular Péclet number according to equation 9 


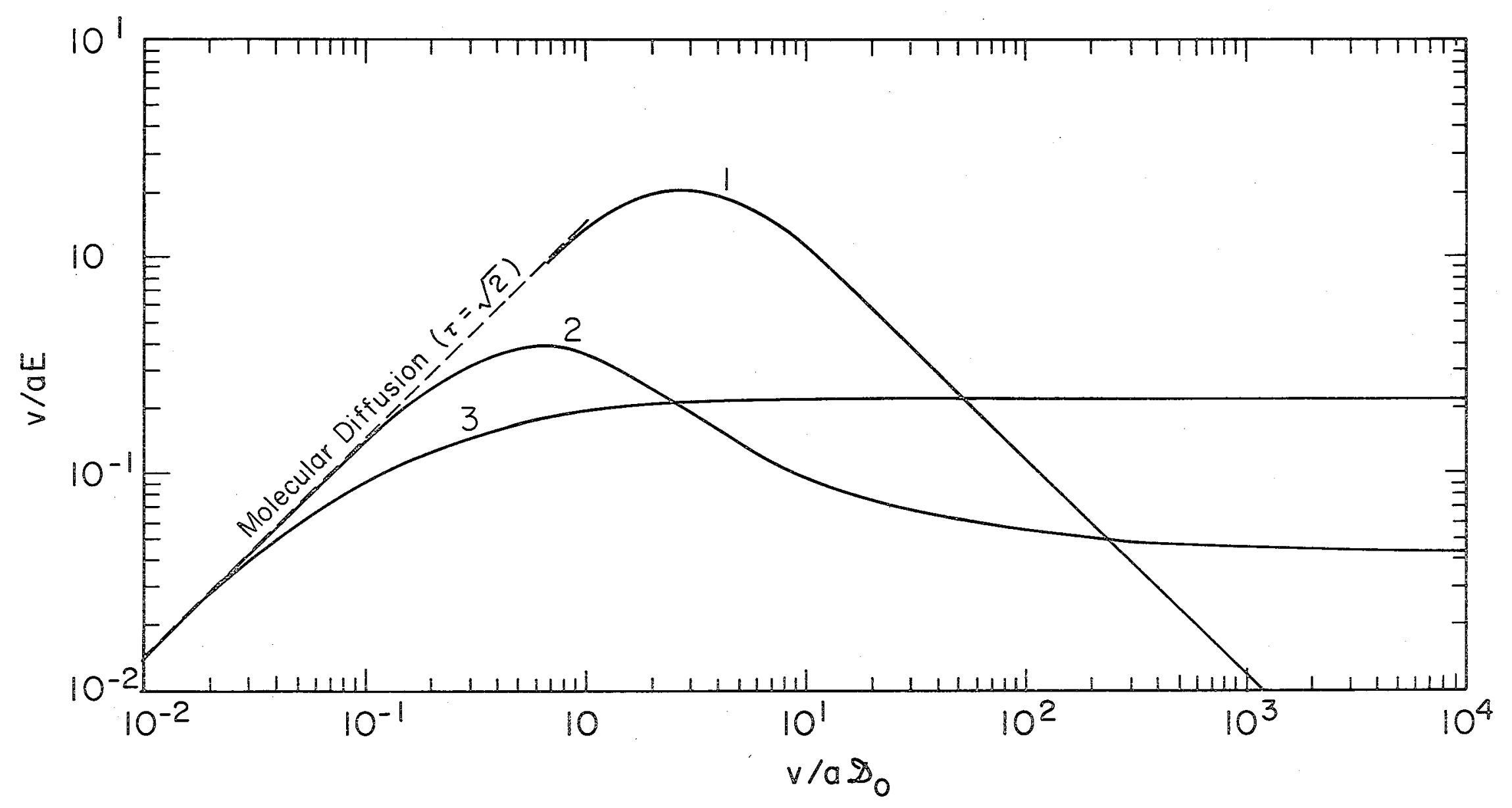

XBL $7811-12811$

Figure 2.2 Dispersion coefficient as a function of the Péclet number: Curve 1) Taylor dispersion in a tube; 2) Gunn (1969) and Miyauchi and Kirckuchi (1975) correlation for creeping flow in a packed bed; 3 ) combination of molecular diffusion and turbulent eddies in a packed bed with $\varepsilon=0.4$. 
and equation 10. The Taylor dispersion coefficient through a tube is included for comparison's sake. Equation 10 will be used in the course of this work for the dispersion coefficient.

\section{Mass-Transfer Coefficients}

The film mass-transfer coefficient is a measure of the local reaction rate in the bed. It is a derived quantity which is not very convenient to measure. The concentration of a reactant far upstream and far downstream of a reactor is more readily accessible to experimental determination. These measurements are correlated by the effective mass-transfer coefficient $k_{m}$. In the mass-transfer controlled reactor under discussion, the definttion of $k_{m}$ is

$$
k_{m}=\frac{v}{a L} \ln \left(c_{F} / c_{L}\right)
$$

These two mass-transfer coefficients are related as has been pointed out by Newman and Tiedemann (34). This relationship can be derived by equating $c_{L} / c_{F}$ from equation 11 to $c_{L} / c_{F}$ given in equation 6 . This manipulation results in

$$
k_{m}=\frac{k_{f}}{B}+\frac{v}{a L} \ln \left\{\frac{B+\frac{D^{\prime}}{B^{2}}(1-B) \exp \left[-\alpha L\left(\frac{1}{B}+\frac{B}{D^{\prime}}\right)\right]}{1+D^{\prime} / B^{2}}\right\}
$$

The experimental $k_{\mathrm{m}}$ measurements can then be corrected by equation 12 to give $k_{f}$. A value for the dispersion coefficient is also needed. Chapter 4 will examine equation 12 in the limit of zero Péclet number. 
Figure 2.3 presents $k_{\mathrm{m}}$ data available in the 1iterature. Each of these data points was collected in a mass-transfer controlled bed with the Reynolds number (v/av) less than one. Both gas and liquid phase data are included. There are two important points to note about this data co11ection.

The lines sketched on this figure are drawn to indicate the asymptotic trends of $k_{\mathrm{m}}$ with the Péclet number $\left(\mathrm{v} / \mathrm{a} D_{\mathrm{o}}\right)$. Clearly, there are different trends. In the lower Pêclet number range, $\mathrm{k}_{\mathrm{m}}$ becomes linearly proportional to $\mathrm{v}$, whereas in the higher Péclet number range $k_{m}$ becomes proportional to the cube root of $v$. This second line is a plot of Wilson and Geankopolis' (52) correlation of their data:

$$
\frac{\mathrm{k}_{\mathrm{m}} \mathrm{d}_{\mathrm{p}}}{\bar{D}_{\mathrm{o}}}=\frac{1.09}{\varepsilon}\left(\frac{\mathrm{vd}_{\mathrm{p}}}{\bar{D}_{\mathrm{o}}}\right)^{1 / 3}
$$

The tabular listing on this figure shows the al product of the bed from which the data were taken. It is seen that most data were taken in relatively shallow beds (recall that aL is $\left.6(1-\varepsilon) L / d_{p}\right)$. The aL values range from 3 (one particle layer) to 29.

These data points can be corrected individually with equation 12 to give the $k_{f}$ values required in the porous electrode equations. This is awkward. It would be more convenient if these results were expressed in an equation form. The Wilson-Geankopolis correlation is 
Figure 2.3 Low Reynolds number mass-transfer coefficients in packed beds. The origina1 publications for the above tabular listing may be found in references (46, 47 , $48,49,50,51,52,53)$ respectively.

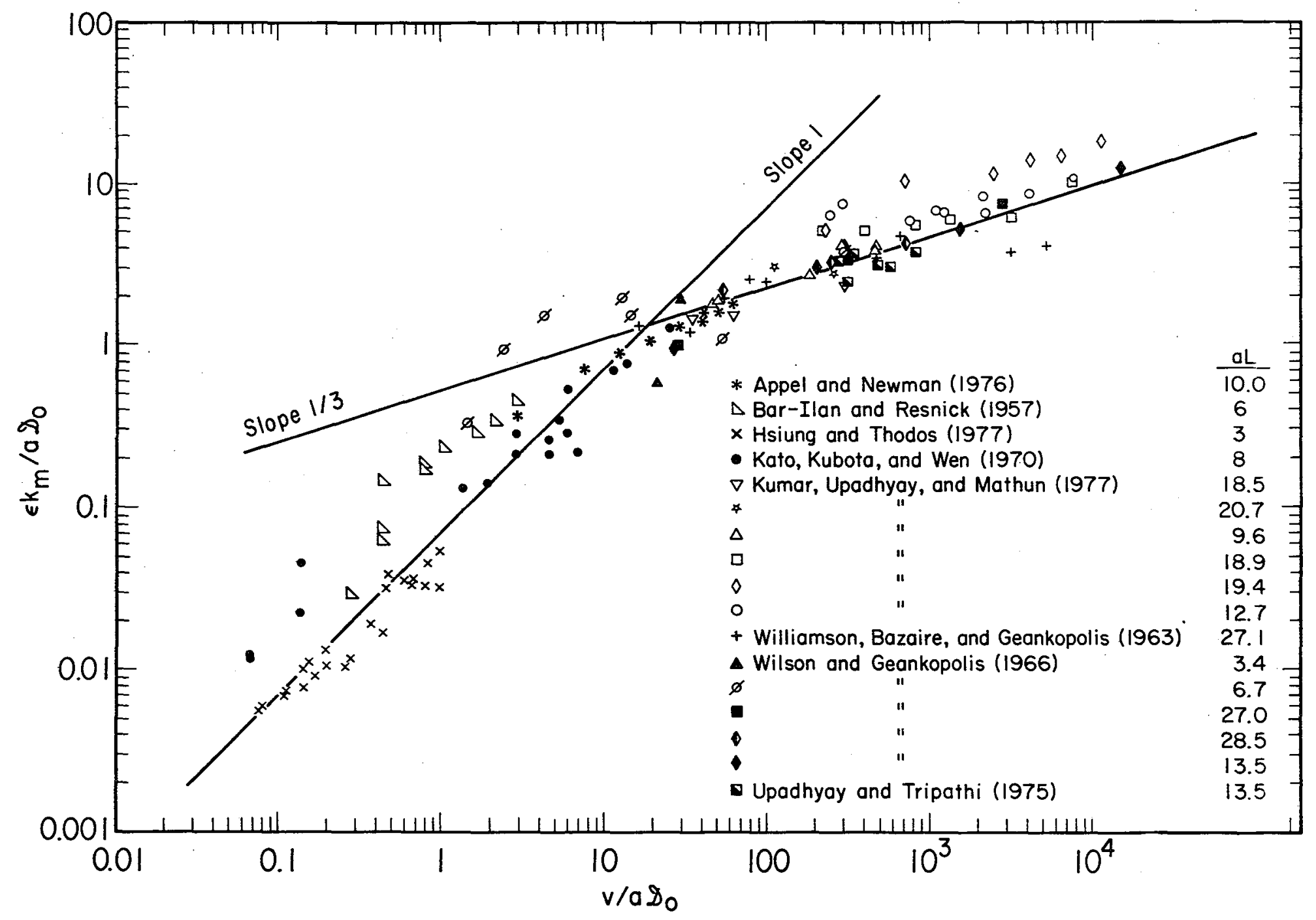

XBL 7811-12876 
valid in the higher Pêclet number region, but it overestimates $k_{m}$ as the Péclet number decreases. Furthermore, it shows the wrong functional dependence. One goal of this thesis is to provide a correlation for the low Reynolds number mass-transfer coefficient. For illustrative purposes at this point, let the effective Sherwood number (the dimensionless $k_{m}$ ) be given by

$$
\frac{1}{\mathrm{Sh}_{\mathrm{B}}}=\frac{1}{1.20 \mathrm{Pe}_{\mathrm{B}}}+\frac{1}{0.914+1.017\left(\frac{\varepsilon}{\mathrm{aL}} \mathrm{Pe}_{B}\right)^{1 / 3}}
$$

The arguments and reasoning which substantiate this expression will be developed in the remaining chapters.

Figure 2.4 illustrates a plot of this equation. The high Péclet number data are satisfactorily fit, but the low Pêclet number data are overestimated. The correct asymptotic trend is recovered, however. Chapter 8 discusses why this correlation as written overestimates $k_{m}$ in this region, and a better fitting equation is suggested there after the data of this work have been introduced. It should be reemphasized that equation 14 is only offered pedagogically at this point so that the behavior of the porous electrode equations can be discussed.

Ohmic Considerations as a Design Constraint

The porous electrode equations 6 and 7 can be studied as a function of flowrate and packing depth with the aid of equation 10 for the dispersion coefficient and equations 12 and 14 for the Sherwood number. 
Figure 2.4 Comparison of experimental effective Sherwood numbers with those predicted using a combination of the straight tube asymptotes with $\varepsilon=0.4$ and aL $=15$.

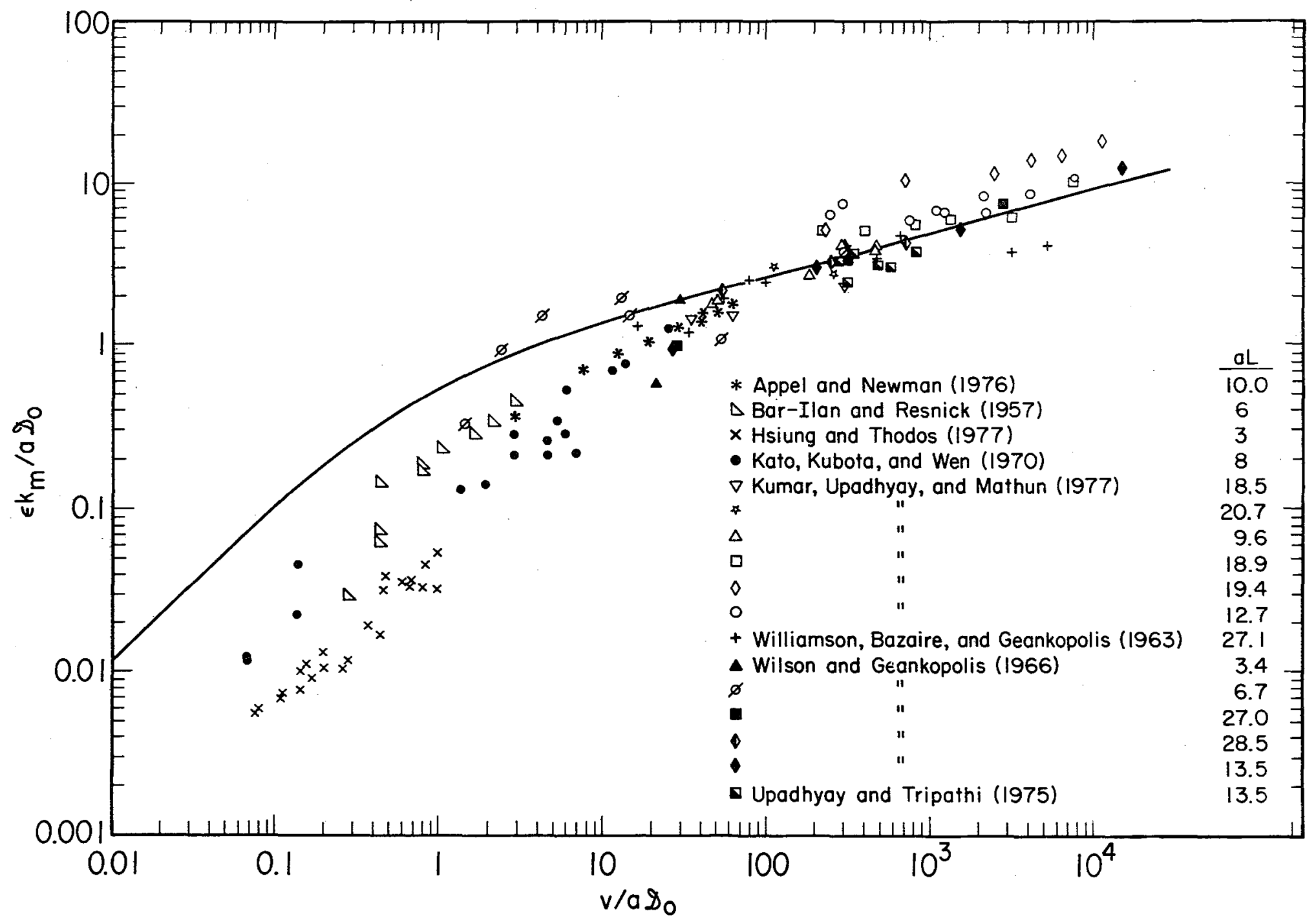

XBL $7812-13629$ 
Newman and Tiedemann (34) have discussed the design constraints that might be imposed on an electrode. (This reference should be consulted for a more detailed presentation.) The conversion of the reactant is a constraint which sets the $c_{L} / c_{F}$ value that must be achieved. The ohmic potential drop across the solution is a second design constraint.

The potential variation in the solution across the electrode is sketched schematically in figure 2.5 for a cathodic bed. There is a larger electrical driving force at the inlet to the reactor $(x=0$, nearest to the counterelectrode) than at the outlet. Consider the reactor which is to be designed to carry out a specific electrochemical reaction. If the potential variation in the solution becomes large enough, undesired secondary reactions may become significant.

As an example, consider a waste stream containing $660 \mathrm{mg} \mathrm{Cu} / \mathrm{l}$ with a $\mathrm{pH}$ of two. Suppose this $\mathrm{Cu}$ is to be removed in a porous electrode. Hydrogen evolution is the undesired secondary reaction. Figure 2.6 is a Pouxbaix diagram for this reaction. From it one can see there is approximately $0.33 \mathrm{~V}$ available to drive this reaction until the evolution of $\mathrm{H}_{2}$ is thermodynamically possible. One can choose the operating conditions of the reactor such that the electrical driving force at the inlet of the reactor does not exceed this value. In the absence of specific kinetic information, this front-face potential is arbitrarily halved, and the resulting $0.16 \mathrm{~V}$ is set equal to the electrical driving force at the exit of the electrode. This establishes a value for the maximum allowable solution ohmic potential drop. 


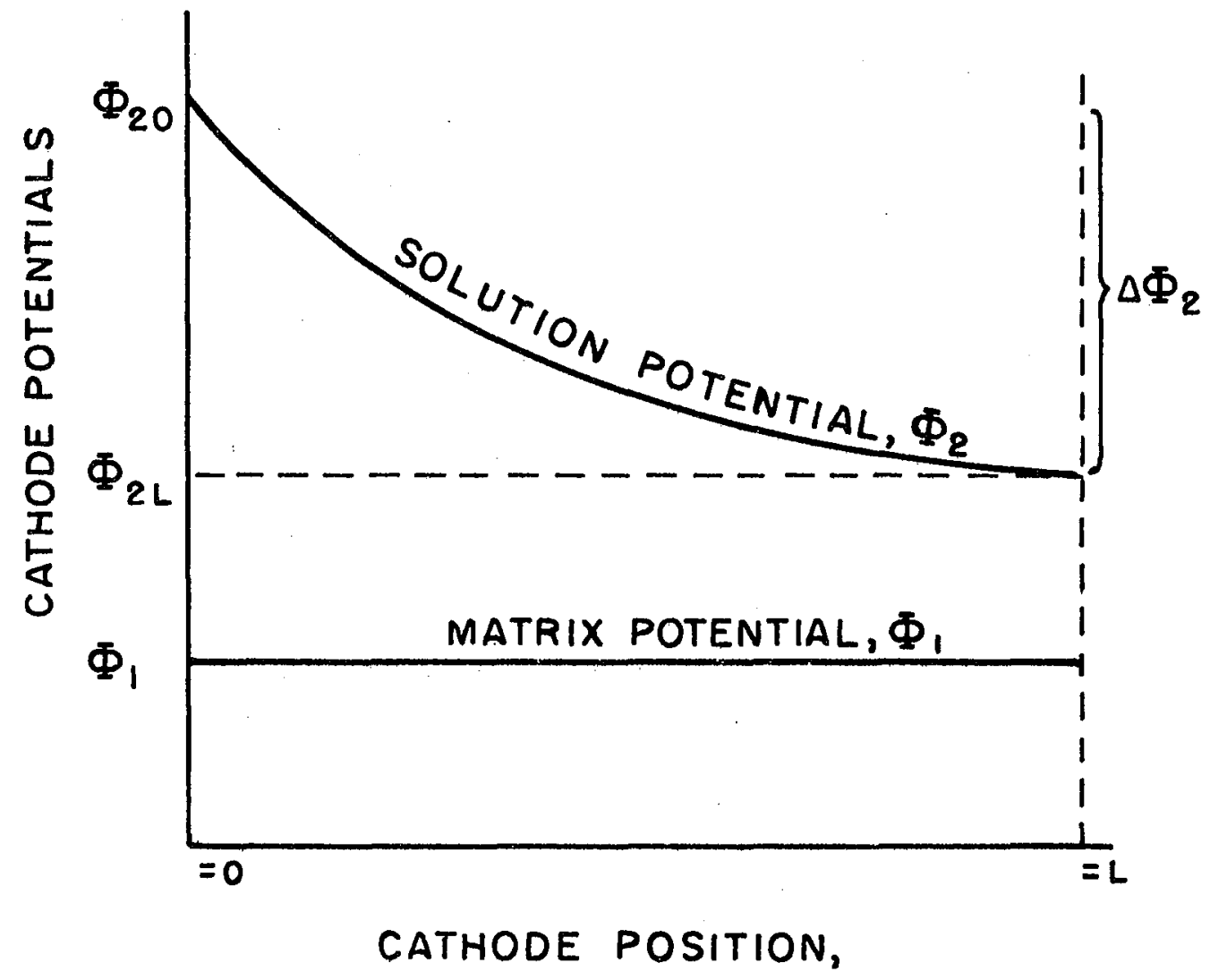

$X B L 736-6256$

Figure 2.5 Solution-phase and solid-matrix-phase potentials, as functions of cathode position. 


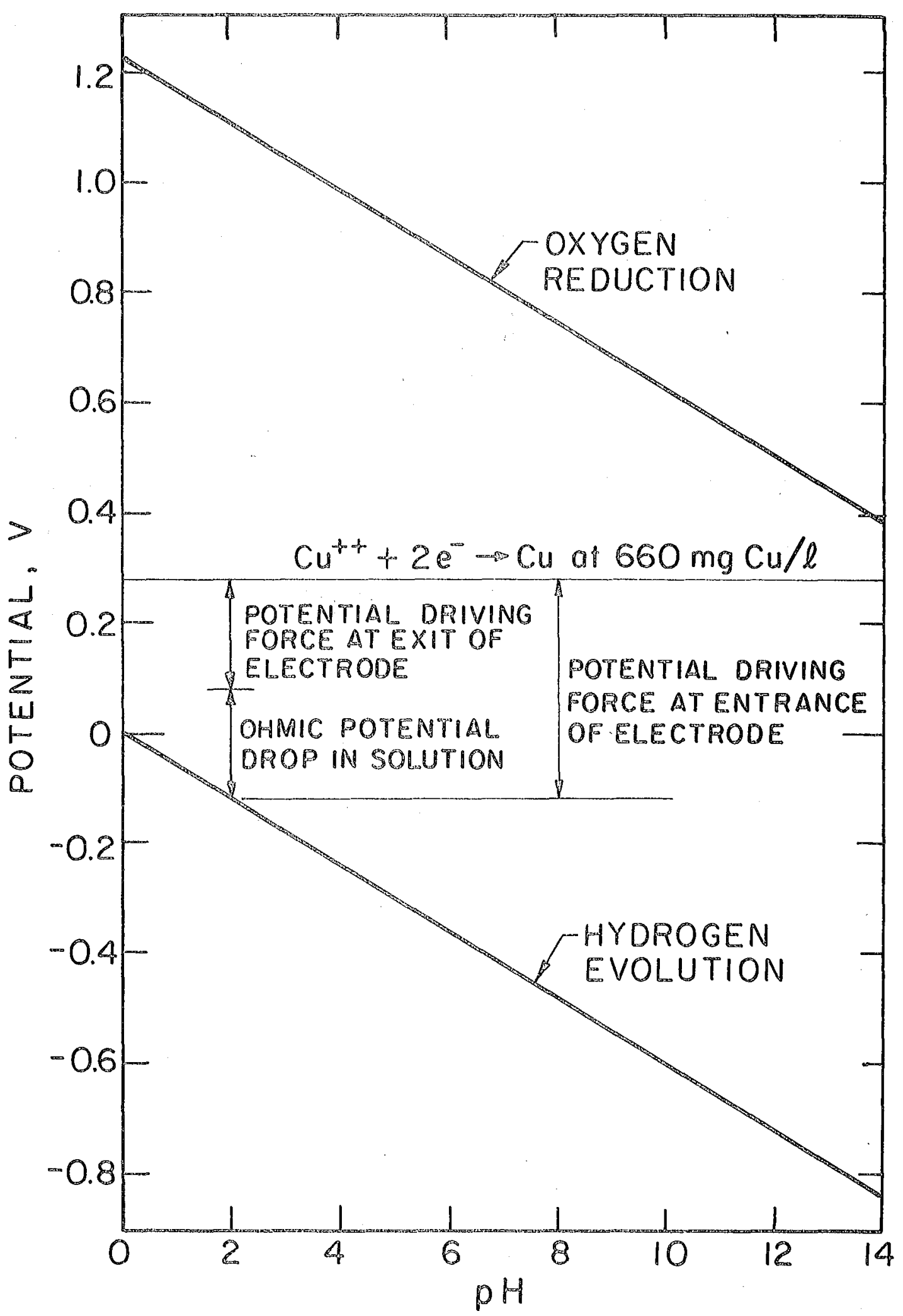

XBL 7811-12880

Figure 2.6 Pourbaix diagram to illustrate ohmic considerations in a porous electrode. 
Care must be taken in establishing the electrical driving force at the rear of the reactor. If it is set too low, a limiting-current condition may not be sustained by the available driving force. A kinetic expression for the reaction would be helpful in establishing the minimum allowable driving force at the reactor exit. The Trainham and Newman (35) analysis incorporates the kinetics.

Newman and Tiedemann (34) have presented an ingenious graphical technique to calculate the packing depth and flowrate required to satisfy the conversion and ohmic drop criteria. They assumed that dispersion was unimportant $\left(D^{\prime} \approx 0\right)$ and utilized the $k_{m}$ data in a graphical form. Their results will not be duplicated here, but rather an alternative approach is presented which includes the dispersive flux and assumes a $k_{m}$ correlation equation is available.

Figure 2.7 is a plot of the dimensionless solution potential drop as a function of the Péclet number. The curves are parameterized in ${ }_{\mathrm{L}}$ corresponding to the design conversion for the reactor. The flowrate required to meet the design specifications can be found from the abscissa since the ordinate and $\theta_{L}$ are set. Equations 11 and 14 are then used to calculate the bed depth.

The computer program written to generate figure 2.7 is presented in Appendix B.

For typical values of $n, \varepsilon, D_{0}, \kappa_{0}, c_{F}$, and $\Delta \Phi_{2}$, the ordinate may vary from 10 to $10^{6}$. Figure 2.7 indicates that the corresponding Péclet numbers will roughly vary from 1 to $10^{3}$. The bed depth will 
Figure 2.7 Design plot for a limiting-current porous electrode given the maximum allowable solution potential drop and the required conversion.

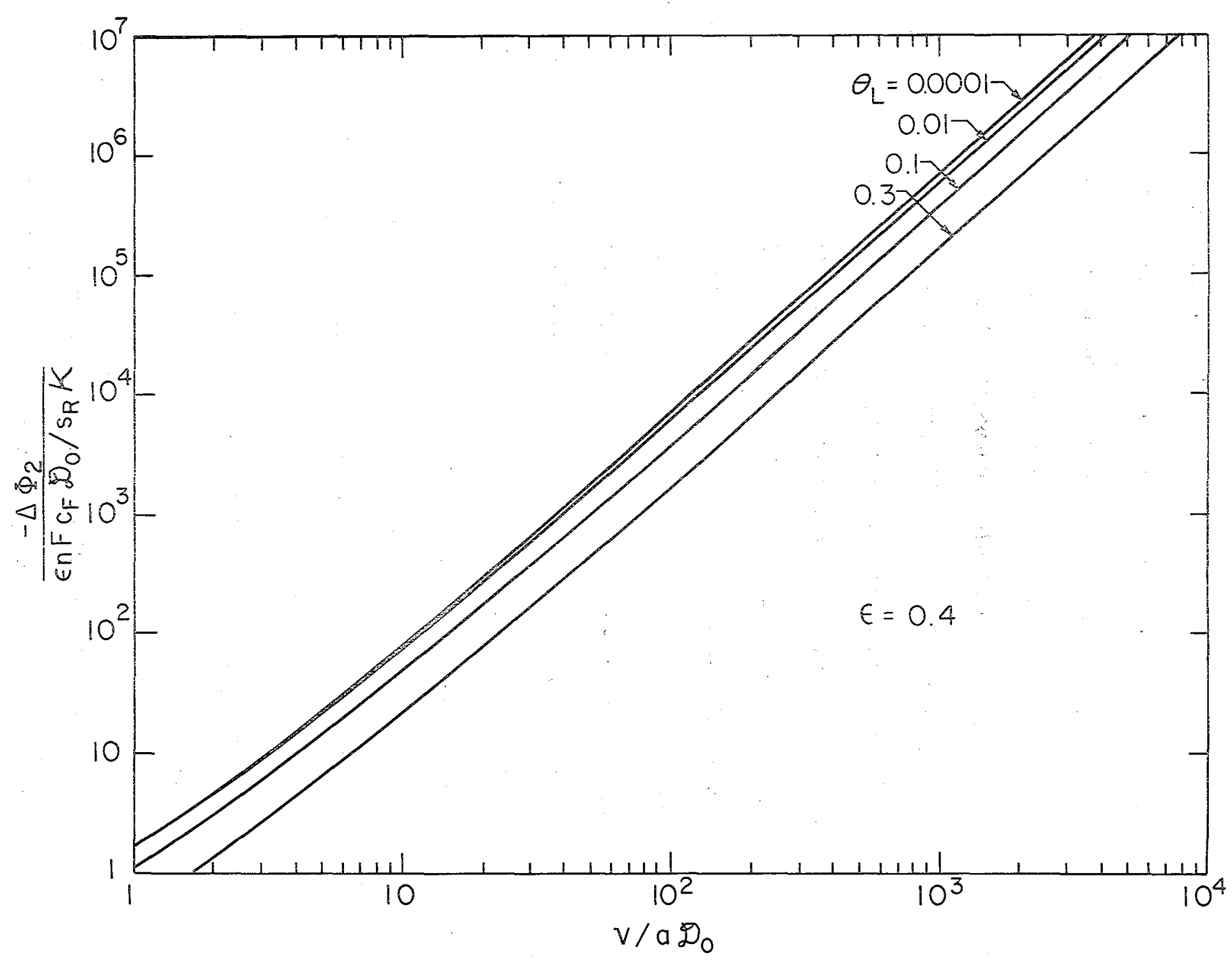

$X B L 7811-6181$ 
depend upon the value set for the conversion. It is clear that the packing depth required to achieve a certain conversion increases with the Péclet number. These two observations show that more experimental $k_{m}$ data in the lower Péclet number range are required. It is precisely in this region of Péclet numbers that $k_{m}$ is changing from a cube root dependence to a linear dependence on $v$. It is a1so clear that data are needed for deeper beds than those currently reported in the 11terature.

The purpose of this thesis is to understand better the $\mathrm{k}_{\mathrm{m}}$ behavior in this low Reynolds number region. 
Chapter 3

Mass Transfer at High Péclet Numbers for Creeping Flow in a Packed-Bed Reactor

\section{Abstract}

An isotropic homogeneous packed bed reactor is modeled as an array of sinusoidal periodically constricted tubes (PCT). The effective asymptotic-bed Sherwood number has been calculated for mass transfer at large Péclet number with a constant wall concentration and creeping-flow hydrodynamics. The bed friction factor has also been calculated. The results for these macroscopic bed quantities depend upon two ratios of the microscopic PCT period length, average radius, and sinusoida1 amp1itude. 
Scope

The mass-transfer rate occurring across a packed bed reactor can be predicted a priori if the exact geometry of the flow channels is known. This is usually impossible except for a uniformly structured bed. It then becomes necessary to introduce microscopic channel models for the bed. The simplest model considers the bed to be an array of straight cylinders. A higher order approximation accounts for the fact that the straight conduit model cannot reproduce the contortions the fluid must pass through in the bed. A periodically constricted tube (PCT) model of a bed, however, is a step in this realization. The converging, diverging character of the flow in these tubes is a better approximation to the true nature of the flow in the actual bed.

Using the PCT model for the flow channels in a bed, the appropriate governing equations can then be solved for the Sherwood number of the bed. Specifically, the Navier-Stokes equations must first be solved for the velocity field which is then used in the convective diffusion equation to solve for the reactant concentration profile. This paper presents results for the friction factor and the Sherwood number of a deep bed modeled as an array of sinusoidal PCT. Creeping flow has been assumed, and the wall concentration of the reactant is constant through the depth of the bed. Since the Schmidt number for liquid reactants is high, a large reactant Péclet number is assumed in the analysis. 


\section{Conclusions and Significance}

A homogeneous, isotropic packed bed reactor can be modeled as an array of periodically constricted tubes. By neglect of entrance region effects, the governing equations for fluid flow and mass transport need only be solved in a single period due to the assumed homogeneicy of the bed.

Interior collocation on a finite-difference gxid was used to reduce the creeping flow stokes stream function equation in a sinusoidal PCT to a set of coupled, fourth order, ordinary differential equations. This approach is much more economical than solving the fullelliptic partia1 differential equation by overrelaxation.

At a high reactant Péclet number in the fully developed masstransfer region, the convective diffusion equation for the reactant in a PCT can be reduced to a Graetz-1ike eigenvalue problem. This technique is valid for laminax flow in any PCT.

Figure 3.9 shows the friction factor, Reynolds number product in creeping flow for a packed bed modeled as an array of sinusoidal PCT. The results depend upon the two dimensionless geometric variables $\mathrm{r}_{\mathrm{A}}$ and $\mathrm{A} / \mathrm{r}_{\mathrm{A}}$ (figure 3.2 ). As the average wall radius decreases or as the amptitude increases, the product increases.

Results are presented in figure 3.11 for the asymptotic Sherwood number of a deep bed reactor with a large reactant Pêclet number in creeping flow. Again the results depend upon the two dimensionless geometric variables. The bed Sherwood number exhibits different behavior in the amplitude-radius ratio $\left(A / r_{A}\right)$ for small and large 
values of $r_{A}$. In beds of long skinny tubes $\left(\operatorname{small} r_{A}\right)$, the Sherwood number increases with $A / r_{A}$, whereas for larger ${ }^{r_{A}}$ this trend reverses itself.

\section{Introduction}

The flow channels in a randomly packed bed defy an analytic expression. To predict a priori the transfer rates in a bed, it then becomes necessary to resort to empirical correlations or, alternatively, to a microscopic model for the flow channels. The appropriate rate equations can be solved within the framework of the mode1 to predict the performance of a bed. Of course, the structured formulation of a microscopic channel model is a framework to understand better the empirical correlations.

The simplest model of a bed considers the flow channels to be an array of straight tube capillaries embedded in an impermeable matrix. Sheidegger ( 23 ) and more recently Dullien ( 14 ) have provided a review of this approach. Such a first order approach cannot, however, without Introducing another parameter, satisfactorily correlate experimental data. The straight streamlines which result from applying the capillary model seem to be an inappropriate approximation to the twisting, converging, diverging character of the flow in an actual bed. This undulating character of the flow can have tremendous consequences on the bed pressure drop and the fluid-to-particle (or vice-versa) mass transfer rates.

Petersen's ( 28 ) work suggested that the flow channels in a bed can be modeled as an array of periodically constricted tubes (PCT). 
Michaels ( 54 ), Houpeurt ( 55 ), Batra et al。 (56), Dullien and Azzam ( 57 ), Payatakes et a1. $(29,30)$ and Sheffield and Metzner ( 58 ) have contxibuted to this line of thought. The converging, diverging nature of the flow in these nodel tubes is a better approximation to the true character of the flow in the bed. Payatakes et a1. have argued by statistical and heuristic means that the problem of modeling the flow behaviox in an array of randomly sized PCT reduces to considering a single dimensionjess PCT. They've presented a technique to calculate the PCI model parameters.

Having a flow channel model in hand, one can then proceed to calculate the pressure $d r o p$ and the reactant concentration profile across a packed bed reactor. Specifically, the Navier-Stokes equation must be solved first for the velocity field (neglecting free convection); and then the convectivemiffusion equation must be solved for the concentration profile of each reactant. Payatakes et al. have outlined a technique for solving the ful1 Navier--Stokes equations in a PCT. No work to the authors' knowledge has been done on the mass transfer problem in a packed bed reactor modeled as an array of these PCT. In this work we have calculated the asymptotic, creeping-flow Sherwood number (based on a logrithmic mean concentration driving force) for a single limiting reactant with a high Péclet number. Physically, these restrictions correspond to a liquid reactant flowing through a deep bed at a low Reynolds number. The reactant wall concentration is assumed constant throughout the length of the reactor, corresponding to a limiting current condition. 
The behavior of the effective mass-transfer coefficient through a packed bed depends upon the flow regime. For a deep bed, the effective mass-transfer coefficient in creeping flow will become independent of the velocity. This is in contrast to the entry region where the transfer rate is proportional to the velocity to the $1 / 3$ power. The entry region has an effective transfer coefficient larger than that for deeper beds. Calculating the deep-bed asymptotic Sherwood number thus gives a lower limit to the expected behavior. The horizontal line of figure 3.1 shows the nature of this Sherwood number. The dashed lines indicate entry-region coefficients for two different sized beds. The line marked $\mathrm{aL}=10$ is the Wilson-Geankopolis ( 52 ) correlation. The left and right hand sides of this figure indicate schematically regions where axial diffusion and turbulent convection, respectively, become important. The turbulent region line is a plot of the Bird et a1. ( 59 ) correlation while the low Péclet number region is a plot of S $\phi$ rensen and Stewart's ( 12 ) calculations for a simple cubic packed bed of spheres.

\section{Mathematical Modeling}

Creeping Flow in a PCT

The PCT considered is generated by the surface of revolution of a cosine function about the axis of symmetry as shown in figure 3.2. A11 lengths are made dimensionless with the period of oscillation $\ell$. The creeping-flow equations are to be solved in this geometry. Because no inertial effects are present and the tube wall is axially symmetric 


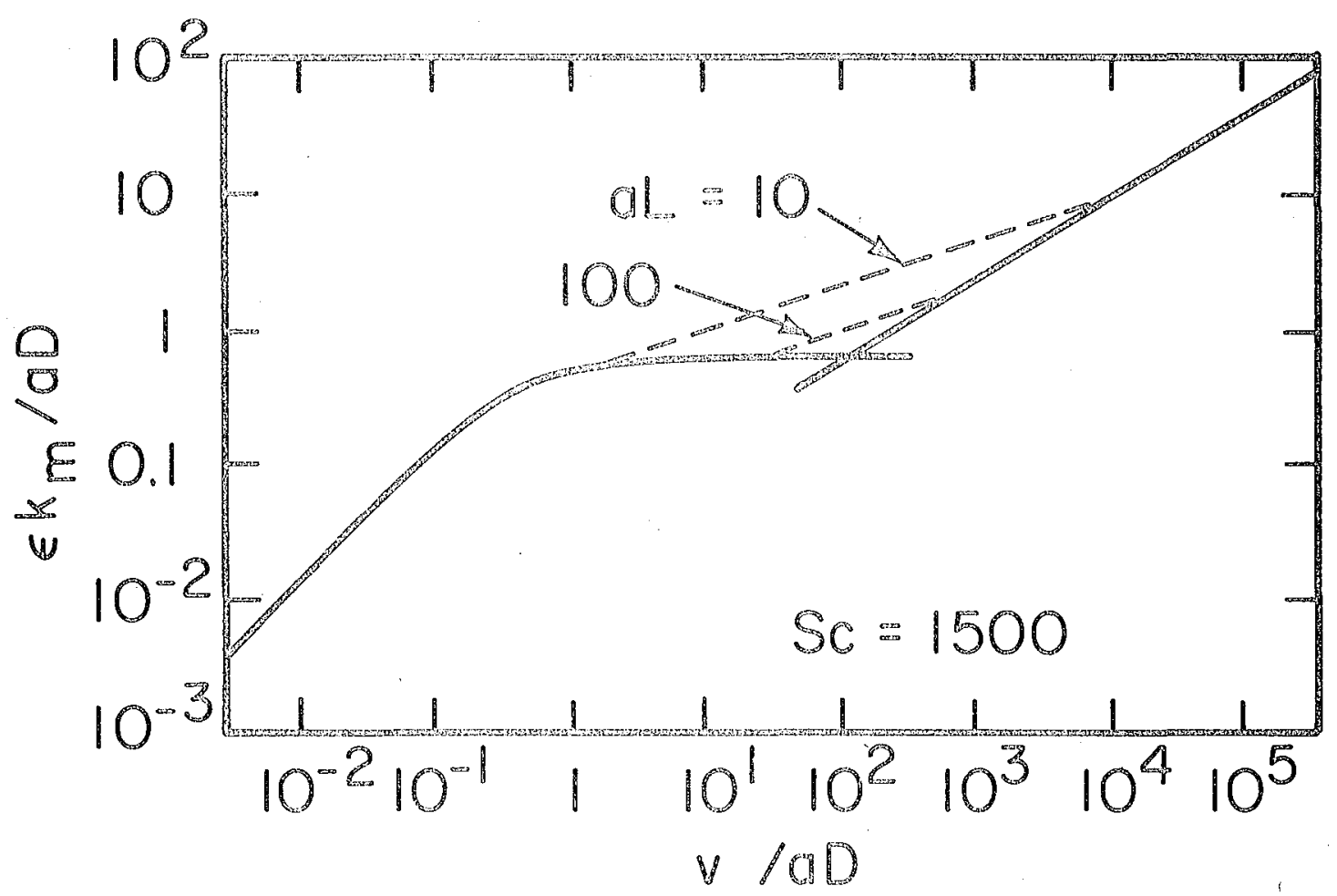

XBL. 7811 12883

Figure 3.1 Expected behavior of bed Sherwood numbers. 


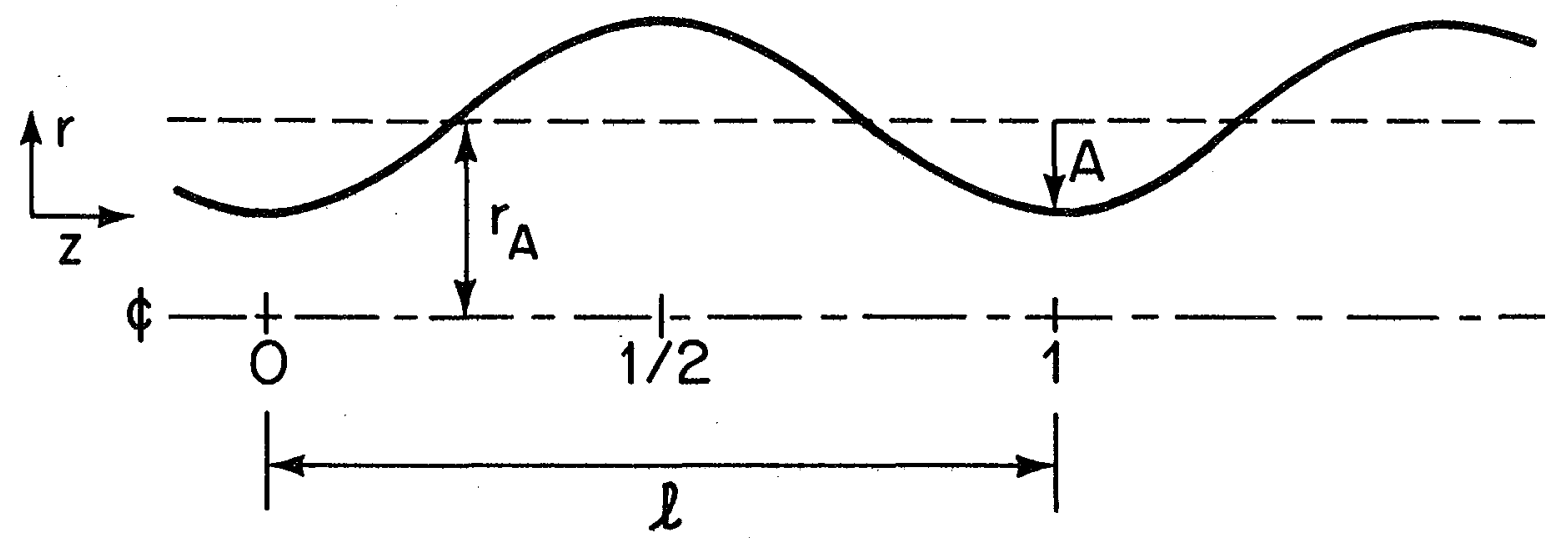

XBL 7811-12884

Figure 3.2 The wall of a PCT generated by $r_{w}(z)=r_{A}-A \cos (2 \pi z)$. All lengths are made dimensionless with respect to the period length $\ell$. 
at $z=0,0.5$, and 1 , the radial velocity $v_{x}$ will be zero at these same positions, It then follows the streamwise velocity $v_{\xi}$ will be an even periodic function of $z$ with the same frequency as the wall oscillation. These considerations make it clear that the governing equations need be only solved in $0 \leq z \leq 0.5$ for this particular geometry.

A packed bed is modeled as an array of these PCT. The fluid approaches the bed at a superficial approach velocity $v$. The average dimensional velocity $\left\langle\mathrm{v}_{\mathrm{Ad}}\right\rangle$ through each tube is defined such that the flow rate in each tube is equal to ${ }^{<\mathrm{V}_{\mathrm{Ad}}}>\pi \mathrm{x}_{\mathrm{Ad}}^{2}$ where $\mathrm{r}_{\mathrm{Ad}}$ is the length averaged dimensional radius. Geometrical considerations show that $\left\langle\mathrm{v}_{\mathrm{Ad}}\right\rangle$ can be written in terms of the approach velocity as

$$
\left\langle\mathrm{v}_{\Delta d}\right\rangle=\frac{\mathrm{v}}{\varepsilon}\left[1+\frac{1}{2}\left(\mathrm{~A} / \mathrm{r}_{\mathrm{A}}\right)^{2}\right]
$$

where $A$ is the dimenstonless wall oscillation amplitude. The governing equations need be solved in a single PCT. These results can then be applied to the entire bed due to the assumed homogeneity and periodicity of the structure.

The dimensionless, incompresstble NaviermStokes equations for creeping flow with axial symetry can be reduced to a single, linear, fourth order partial diffexential equation by introducing the normalized stream function $\psi$ as

$$
E^{4} \psi=0
$$


where

$$
\begin{aligned}
E^{2}=\frac{\partial^{2}}{\partial r^{2}} & -\frac{1}{r} \frac{\partial}{\partial r}+\frac{\partial^{2}}{\partial z^{2}} \\
v_{z} & =\frac{r_{A}^{2}}{2 r} \frac{\partial \psi}{\partial r} \\
v_{r} & =-\frac{r_{A}^{2}}{2 r} \frac{\partial \psi}{\partial z} .
\end{aligned}
$$

The stream function equation is to be solved subject to the boundary conditions

$$
\begin{aligned}
& \left.\begin{array}{c}
\psi=0 \\
\frac{\partial}{\partial r}\left(\frac{1}{r} \frac{\partial \psi}{\partial r}\right)=0
\end{array}\right\} \quad r=0 \\
& \left.\begin{array}{rl}
\frac{1}{r} \frac{\partial \psi}{\partial r} & =0 \\
\psi & =1
\end{array}\right\} \quad r=r_{w}(z)
\end{aligned}
$$

and a periodicity condition

$$
\frac{\partial^{(n)}}{\partial z^{(n)}} \psi(r, z)=\frac{\partial^{(n)}}{\partial z^{(n)}} \psi(r, z \pm m) n, m=0,1,2, \ldots
$$

The boundary conditions of equation 4 state that at the centerline i) the radial velocity is zero, ii) the axial velocity is symmetric, and at the wall iii) there is no slip on the axial velocity, and iv) the flow rate at each cross section is a constant, here referred to a straight cylinder of radius $r_{A}$. 
No analytic solution for equations 1,4 , and 5 could be found. Interior collocation on a finte odifference grid was used to generate an approximate solution. The collocation approximation technique is exanined by Finlayson ( 60 ), Villadsen ( 61), and Villadsen and Stewart ( 62 ).

A transformed xadial coordinate $\eta$ is introduced by

$$
n=r / r_{w}(z)
$$

The boundary conditions of equation 4 along the wall are then transferred to the coordinate curve $\eta=1$. In this new coordinate, the interior collocation technique on a finite-difference grid can be used to approximate the hydrodynamics. Assume a solution for the normalized stream function of the form

$$
\psi(n, z)=2 \eta^{2} \ldots \eta^{4}+\sum_{k=1}^{N C P} \eta^{2}\left(1-n^{2}\right)^{2} A_{k}(z) \phi_{k-1}\left(n^{2}\right)
$$

The first two terms on the right side represent the Hagen-Poiseuille solution. The summation of terms can then be considexed as a correction function to the basic parabolic flow. The functions $\phi_{k-1}\left(\eta^{2}\right)$ in the summation term can be any complete set of functions. The weighting factor $\eta^{2}\left(1-\eta^{2}\right)^{2}$ assuxes the correct behaviox of the solution at the boundary points $\eta=0$ and $\eta=1$. The coefficients $A_{k}(z)$ are unknown functions of $z$ to be determined subject to the boundary conditions 


$$
\begin{aligned}
& A_{k}^{\prime}(0)=A_{k}^{\prime \prime \prime}(0)=0 \\
& A_{k}^{\prime}(0.5)=A_{k}^{\prime \prime \prime}(0.5)=0 .
\end{aligned}
$$

These conditions result from the periodic, symmetric tube wall. In non-creeping flow, these coefficients would not 1dentically equal zero but some constant which must be determined as part of the solution.

A friction factor for a packed bed may be defined as

$$
f_{B}=\frac{36 \varepsilon^{3}}{a}\left(\frac{-\Delta P_{B}}{L}\right) \frac{1}{\rho v^{2}} .
$$

A porosity dependence has been explicitly incorporated into this definition. For creeping flow, the product of the Reynolds number and the bed friction factor is a constant given by

$$
\begin{gathered}
f_{B} \operatorname{Re}_{B}=72\left(\frac{2 \varepsilon}{a_{A d}}\right)^{2}\left[1+\frac{1}{2}\left(A / r_{A}\right)^{2}\right] \int_{0}^{1}\left(\frac{r_{A}}{r_{w}}\right)^{4}\left\{1+\sum_{k=1}^{N C P} A_{k}(z)\right. \\
\left.\left[2 \phi_{k-1}(0)-\phi_{k-1}^{\prime \prime}(0) / 2\right]\right\} d z .
\end{gathered}
$$

This equation was derived by integrating the pressure gradient in the Navier-Stokes equations over a period at the centerline. The left side of equation 10 depends upon the macroscopic bed quantities while the right side depends upon the microscopic model parameters ${ }_{\mathrm{A}}$ and $\mathrm{A} / \mathrm{r}_{\mathrm{A}}$ only. 
Convective Diffusion Equation at High Péclet Numbers

The dimensionless, steady-state, convective-diffusion equation for a single limiting reactant can be written in generalized vector notation as

$$
\underline{\mathrm{v}} \cdot \nabla \mathrm{C}=\frac{2 \mathrm{r} A}{\mathrm{Pe}} \nabla^{2} \mathrm{C}
$$

This equation with a creeping flow velocity profile is to be solved in the far downstream region of a PCT for the asymptotic solution as $\mathrm{Pe} \rightarrow \infty$. Solving this equation in a straight tube after neglecting diffusion in the axial direction results in the we11-known Graetz solution. At high $\mathrm{Pe}$ it is also valid to neglect diffusion parallel to the streamwise velocity in a PCT.

It is convenient to solve equation 11 in a transformed coordinate system $(\psi, \xi, \theta)$ (figure 3.3 ). The $\psi$ coordinate is constant along streamlines and is found directly from the stream function. The $\xi$ direction is parallel to the streamwise velocity at all positions and is scaled such that $\xi=0$ at the beginning of a period and $\xi=1$ at the end. It is defined implicitly by $(\nabla \psi) \cdot(\nabla \xi)=0$. The angular coordinate $\theta$ has its usual meaning. In this coordinate system, diffusion will be important in the $\psi$ direction and negligible in the $\xi$ direction, at high Péclet numbers.

With neglect of diffusion in the $\xi$ direction, equation 11 can be written as 


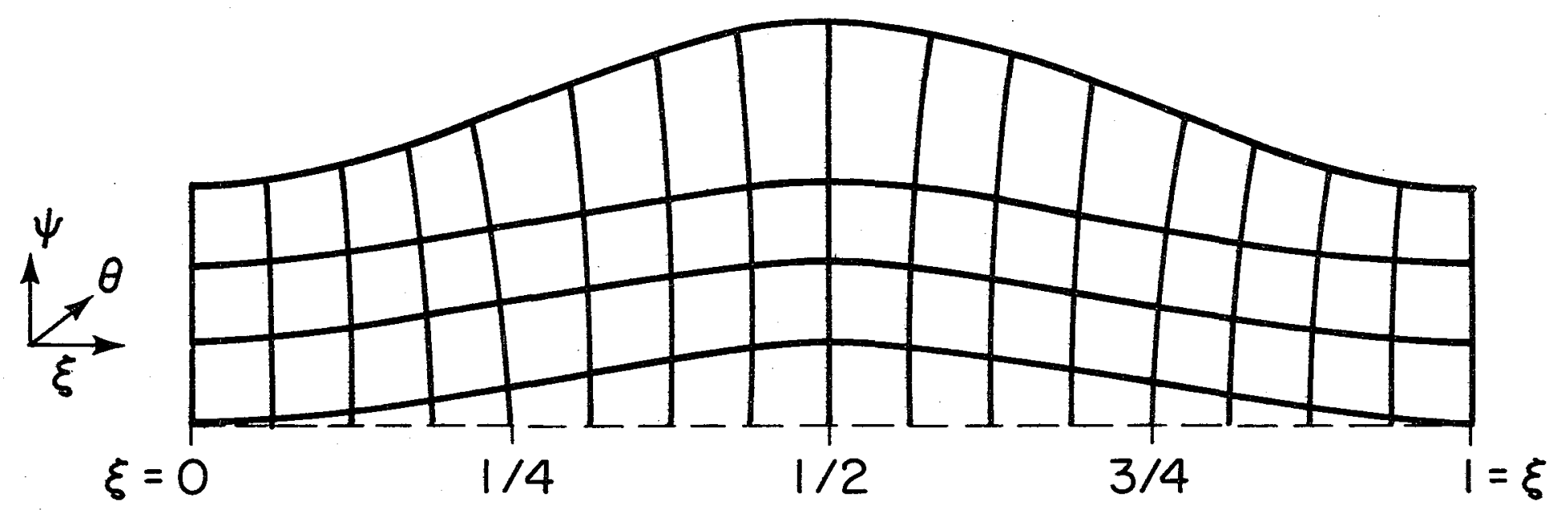

XBL $7811-12885$

Figure 3.3 The $(\psi, \xi, \theta)$ coordinate system. 


$$
\frac{v_{\xi}}{h_{\xi}} \frac{\partial C}{\partial \xi}=\frac{2 r}{P e} \frac{1}{h_{\theta} h_{\psi} h_{\xi}} \frac{\partial}{\partial \psi}\left(\frac{h_{\theta} h_{\xi}}{h_{\psi}} \frac{\partial C}{\partial \psi}\right) .
$$

Explicit forms for two of the metric factors can be determined. By inspection $h_{\theta}=r$. Since the stream function represents the amount of fluid flowing in a stream tube between a point and the axis,

$$
\psi=\frac{2}{r_{A}^{2}} \int_{0}^{\psi} v_{\xi} r h_{\psi} d \psi
$$

after appropriate normalization. It follows that the metric factor $h_{\psi}$ is related to the streamwise velocity $v_{\xi}$ :

$$
\mathrm{h}_{\psi}=\frac{\mathrm{r}_{\mathrm{A}}^{2}}{2 \mathrm{r}} \frac{1}{\mathrm{v}_{\xi}}
$$

Equation 12 now becomes

$$
\frac{\partial C}{\partial \xi}=\frac{8}{r_{A} \operatorname{Pe}} \frac{\partial}{\partial \psi}\left(\left(r / r_{A}\right)^{2} v_{\xi} h_{\xi} \frac{\partial C}{\partial \psi}\right)
$$

which applies to any PCT.

Unfortunately, equation 15 cannot be solved by a separation of variables technique. One can, however, formulate a perturbation solution to equation 15 in the deep region of the bed where the entrance effects have been damped. Equation 15 suggests as a first approximation that

$$
\frac{\partial \mathrm{C}}{\partial \xi}=0
$$


at large $\mathrm{Pe}$. This would imply that the concentration is a function of $\psi$ only and is constant along a streamline. Any function of $\psi$ will suffice. The first order term in the perturbation solution should then be a function only of $\psi$. The second order term will then be a diffusive correction function to take into account that the concentration must also be changing in the $\xi$ coordinate. Assume a solution of the form

$$
\mathrm{C}(\psi, \xi)=\mathrm{C}_{1}(\psi)+\mathrm{C}_{2}(\psi, \xi)
$$

Substitution of equation 17 into equation 15 yields

$$
\frac{\partial \mathrm{C}_{2}}{\partial \xi}=\frac{8}{r_{A}^{\mathrm{Pe}}} \frac{\partial}{\partial \psi}\left(\left(\mathrm{r} / \mathrm{r}_{\mathrm{A}}\right)^{2} \mathrm{v}_{\xi} \mathrm{h}_{\xi} \frac{\partial \mathrm{C}_{1}}{\partial \psi}\right)
$$

after neglect of the diffusive term in $\mathrm{C}_{2}$.

In the far downstream region of a PCT, the fractional decrease of concentration through each period must be the same, that is

$$
C(\psi, \xi+1)=C(\psi, \xi) e^{-\beta \ell}
$$

where $\beta$ is independent of position. If we set $C_{2}(\psi, 0)=0$, this means that $\mathrm{C}_{2}$ and $\mathrm{C}_{1}$ are related:

$$
C_{2}(\psi, 1)=C_{1}(\psi)\left(e^{-\beta l}-1\right)
$$

Equation 18 can now be integrated from $\xi=0$ to $\xi=1$, to obtain a Sturm-Liouville eigenvalue problem for the function $C_{1}(\psi)$. 


$$
\begin{gathered}
\frac{d}{d \psi}\left(G(\psi) \frac{d}{d \psi}\right)+\lambda C_{1}=0 \\
G(\psi)=\int_{0}^{1}\left(r / r_{A}\right)^{2} v_{\xi^{h}} \xi^{d \xi} \\
\lambda=\left(1-e^{-\beta l}\right) \frac{r_{A} P e}{8} \cong \beta l \frac{r_{A} P e}{8} .
\end{gathered}
$$

The integral in equation 22 is carried out over the arc length for a constant value of $\psi$ in the integrand. The second identification of $\lambda$ to $\beta l$ in equation 23 is possible since $\mathrm{Pe} \rightarrow \infty$.

Equation 21 is to be solved subject to the conditions

$$
\begin{aligned}
& C_{1}(0)=1 \\
& C_{1}(1)=0 \\
& C_{1}^{\prime}(0)=-\lambda / G^{\prime}(0) .
\end{aligned}
$$

Condition ( $i$ ) is a normalization for the first order solution. Condition (ii) satisfies the limiting reactant constraint of a zero wall concentration. Condition (iii) results from the fact that the concentration must be finite on the centerline, a singular point of equation 21.

The first eigenvalue of equation 21 can be related to the effective Sherwood number for a deep porous bed which is modeled as an array of PCT. A macroscopic mass balance on the reactant over the length of the period can be written in terms of an effective mass-transfer coefficient $k_{m}$ (Newman and Tiedemann, 34 Bennion and Newman, 40 ). The $\beta$ in equation 20 can then be related to this coefficient as 


$$
\beta=k_{m} a / v
$$

With equation 25 and 23, the Sherwood number for a limiting reactant in a deep bed with creeping flow and high Pêclet number can be written as

$$
\mathrm{Sh}_{\mathrm{B}}=\frac{\varepsilon}{\mathrm{a}} \frac{\mathrm{k}_{\mathrm{m}}}{\mathrm{D}}=\lambda\left[\frac{2 \varepsilon}{\mathrm{ar}_{\mathrm{Ad}} \sqrt{1+(1 / 2)\left(\mathrm{A} / \mathrm{r}_{\mathrm{A}}\right)^{2}}}\right]^{2}
$$

Equations 26 and 21 are the main results of this analysis. By means of the perturbation approach, we have demonstrated how the twodimensional convective-diffusion equation in a PCT can be reduced to a Graetz-1ike eigenvalue problem at high Péclet numbers. The first eigenvalue of this problem is simply related to the bed Sherwood number as given in equation 26.

The eigenfunction $C_{1}(\psi)$ generated by the perturbation analysis is a first order approximation to the concentration distribution. It identically satisfies equation 16 and gives the correct integral properties to the correction function $\mathrm{C}_{2}(\psi, \xi)$. The local transfer rate to the wall can be found by differentiation of this profile with respect to the normal distance from the wall. After a change in coordinate system (see next section), the analysis yields

$$
\left.\frac{\partial C}{\partial n}\right|_{W}=-\left.\frac{\sqrt{B(z) r_{W}(z)}}{2 r_{A}} \frac{d C_{1}}{d \rho}\right|_{\rho=1}
$$

where 


$$
B(z)=\left.\frac{\partial v_{\xi}}{\partial n}\right|_{w}
$$

The local wall flux is thus proportional to the square root of the local shear rate. The integral of equation 27 over the surface area of a period is related to the eigenvalue.

The left side of equation 26 depends upon the macroscopic bed quantities a and $\varepsilon$. The right side is a function of PCT geometry and flow regime through the dependence on $\lambda$. The eigenvalues of equation 21 are independent of the Péclet number in creeping flow. Thus, irrespective of curvature effects, the asymptotic Sherwood number is a constant independent of the Péclet number for a deep bed.

\section{Method of Solution}

The unknown coefficients $A_{k}(z)$ in the interior-collocation approximation for the stream function can be determined as follows. Equation 1 in the $(n, z)$ coordinate system is applied to equation 7. (The $\mathrm{E}^{4}$ operator in the $(n, z)$ coordinate system is given in Appendix A). Interior collocation is then used at NCP points in the $\eta$ coordinate. Since the $\eta$ function dependence is a priori postulated through the $\phi_{k-1}\left(\eta^{2}\right)$, this step reduces the partial differential equation to a set of coupled, fourth order, ordinary differential equations for the unknown $A_{k}$. This set of equations is solved on a finite-difference grid in the $z$ coordinate by the method of Newman $(63)$. Legendre polynomials were used for the $\phi_{k-1}\left(\eta^{2}\right)$. The $\eta$ collocation points were chosen to be the zeros of the shifted Legendre polynomials of order $\mathrm{NCP}-1$ 


$$
n_{i}=\sqrt{\frac{x_{i}+1}{2}}
$$

where $x_{i}$ is the zero of the ordinary Legendre polynomial. The wa11, $\eta=1$, was also used as a collocation point.

The eigenvalue problem as posed in equations 21 thru 24 is 111 suited numerically to the $\psi$ coordinate. Equation 21 has two singular points, one at $\psi=0$, the other at $\psi=1$. The singularity at $\psi=0$ presents no problems; however that at $\psi=1$ does. An analysis of equation 21 near the point $\psi=1$ indicates that the first derivative of $C_{1}$ approaches infinity. A change in coordinate will eliminate this singularity. Define a length-like transformation variable $\rho$ as

$$
\psi=2 \rho^{2}-\rho^{4}
$$

Equation 21 and its boundary conditions then transform as

$$
\begin{gathered}
\frac{d}{d \rho}\left(\frac{G(\psi(\rho))}{4 \rho\left(1-\rho^{2}\right)} \frac{d C_{1}}{d \rho}\right)+4 \lambda \rho\left(1-\rho^{2}\right) C_{1}=0 \\
C_{1}(\rho=0)=1 \\
C_{1}(\rho=1)=0 \\
\frac{d}{d \rho} C_{1}(\rho=0)=0 .
\end{gathered}
$$

Equations 29 and 30 were solved by the method suggested by Newman (64) for eigenvalue problems.

The computer programs are given in Appendix $B$. 
Results and Discussion

The hydrodynamic results will be discussed first, followed by the mass transfer problem.

The interior collocation solution technique for stream function required a maximum of nine $(\mathrm{NCP}=9) \quad \eta$ collocation points to insure sufficient accuracy of the solution. It was found that more collocation points were required as the dimensionless wall radius was increased, nine being the maximum for the most extreme case considered $\left(r_{A}=0.5\right.$, $\left.\mathrm{A} / \mathrm{r}_{\mathrm{A}}=0.5\right)$. Since this approximation solution is solved in a generalized $(n, z)$ coordinate system, it facilitates a straightforward calculation for the velocity field in any tube in the shape of a periodic body of revolution. The reduction of the eliptic partial differential equation to a set of coupled ordinary equations is more economical to solve in terms of computer time usage.

A boundary collocation solution technique was also attempted but was discarded. The general solutions (by separation of variables) to equation 1 involve modified Bessel functions of the first kind. Unfortunately these functions do not form a complete set, and the correction function expansion technique similar to equation 7 did not converge.

Figure 3.4 shows a comparison between the creeping flow axial velocity profile calculated here and that reported by Payatakes et a1. for a tube Reynolds number equal to one. The profiles are compared at the minimum and maximum $(z=0.5)$ constriction diameters. The tube wall for these profiles is generated by two parabolas intersecting at $z=0.5$ with their respective minima at $z=0$ and $z=1$. 


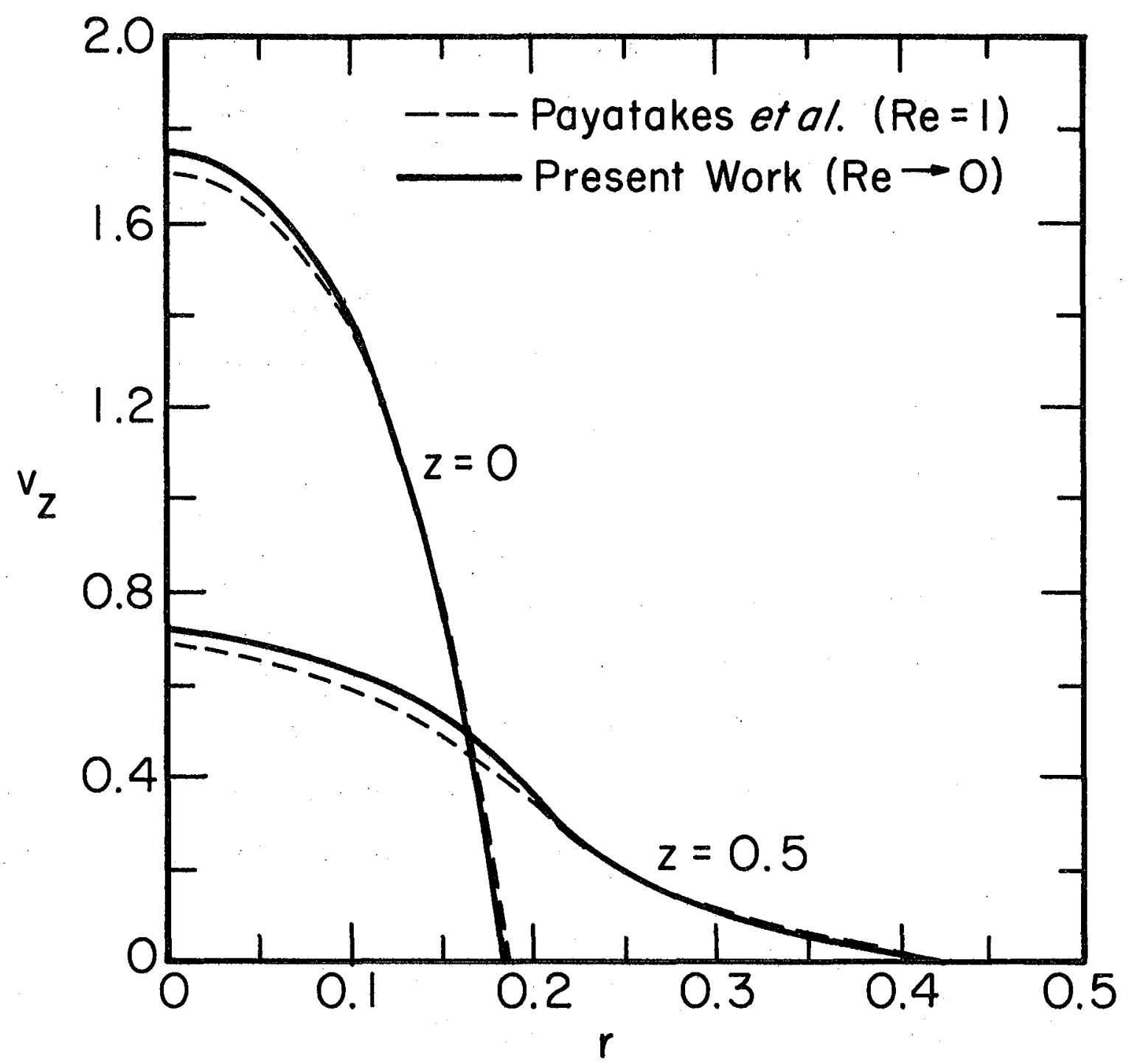

XBL 7811-12882

Figure 3.4 Comparison of calculated axial velocity profiles with those of Payatakes et al. for a parabolic PCT. 
(See figure 1 of Payatakes et al.). The boundary conditions for the $A_{k}{ }^{\prime} s$ in equation 8 were at $z=0$ and 1.0 for this situation. The velocity here is scaled with respect to the average velocity in a tube of constant radius equal to the constriction radius. At the centerline, the viscous flow profile is slightly larger than that of Payatakes et al. calculations. However, near the wall this trend is reversed. The integral of a11 the profiles is equal to a constant defined by the flowrate.

Figures 3.5 thru 3.8 show some typical creeping flow profiles in a sinusoida1 PCT. The two dimensionless geometry groups ${ }^{A}$ and $A / r_{A}$ completely determine the solution behavior. These four figures illustrate the effect on the velocity profiles of manipulating one of these variables with the other held constant. The velocity profiles have been normalized with the average velocity at the average radius.

The effect on the axial and radial velocity profiles of varying the wa11 amplitude at a constant average radius is shown in figures $3 . \dot{5}$ and 3.6. The radial velocity profile is plotted at $z=0.25$. At this position $v_{r}$ attains its maximum value. These figures indicate that at a constant radius the variation in the velocity profiles across a half period becomes more dramatic as the oscillation amplitude increases.

Figure 3.7 and 3.8 illustrate the velocity profiles for a varying wa11 radius at a constant $A / r_{A}$. The effect of the tube geometry is again seen. The radial velocity increases with $r_{A}$ since the velocity of the fluid in the radial direction is proportional to the slope of the wa1l. However, the variations in the axial velocity profiles across 


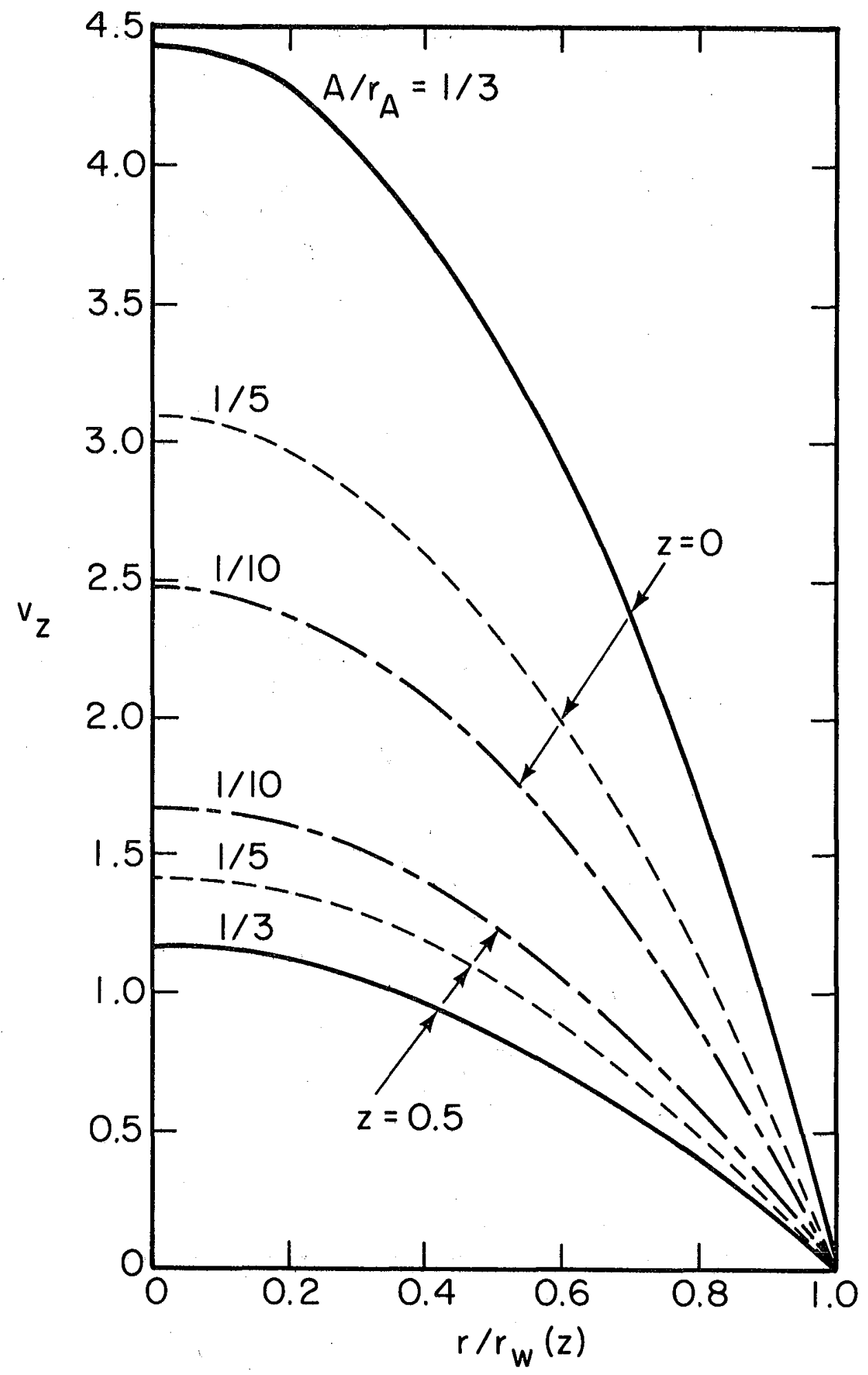

XBL 7811-12886

Figure 3.5 Effect of amplitude/radius ratio on axial velocity profiles for a sinusoidal PCT with $\mathrm{r}_{\mathrm{A}}=0.1$. 


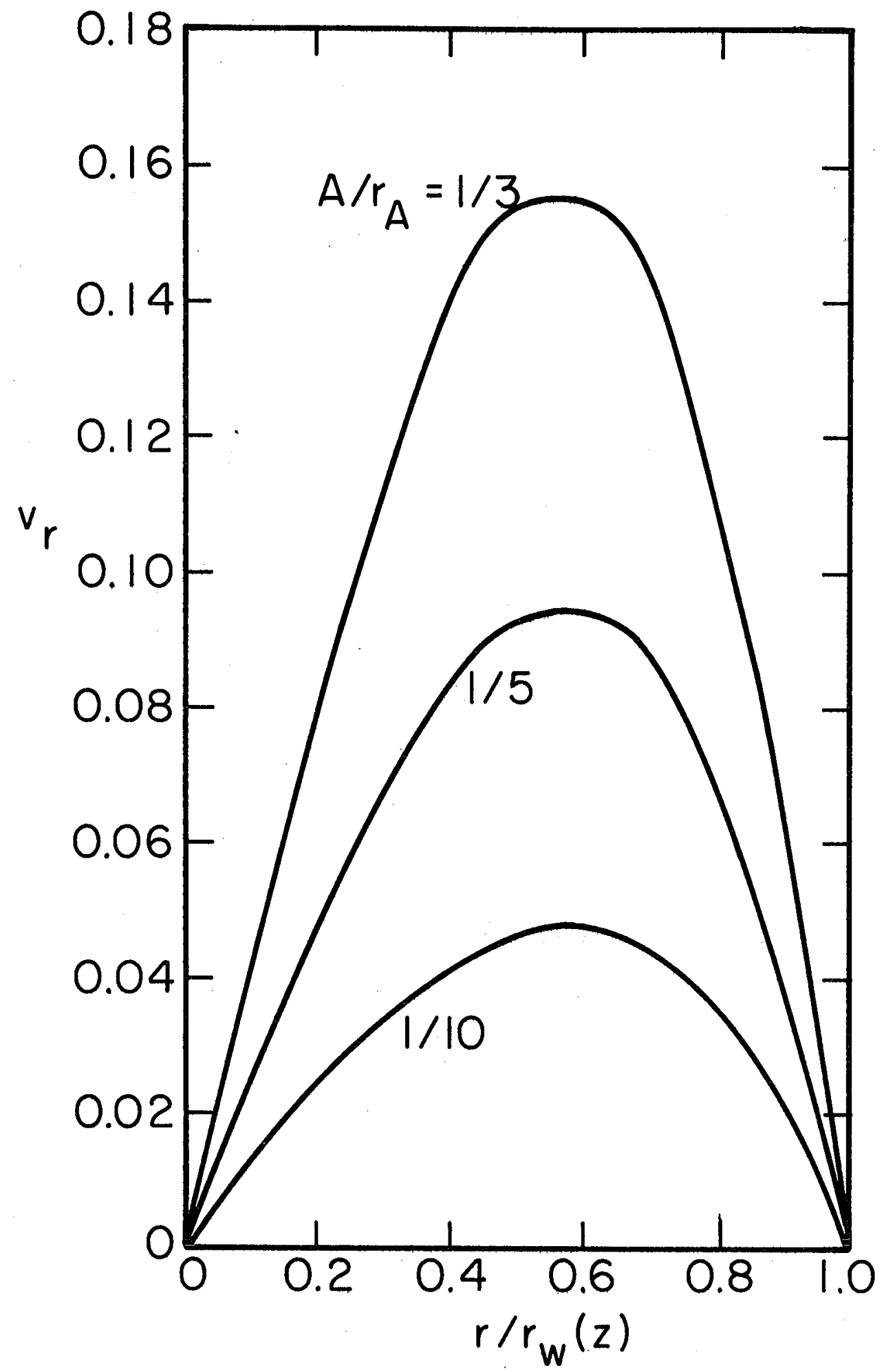

XBL 7811-12881

Figure 3.6 Effect of amplitude/radius ratio on radial velocity profiles in a sinusoidal PCT for $r_{A}=0.1$ at $z=0.25$. 


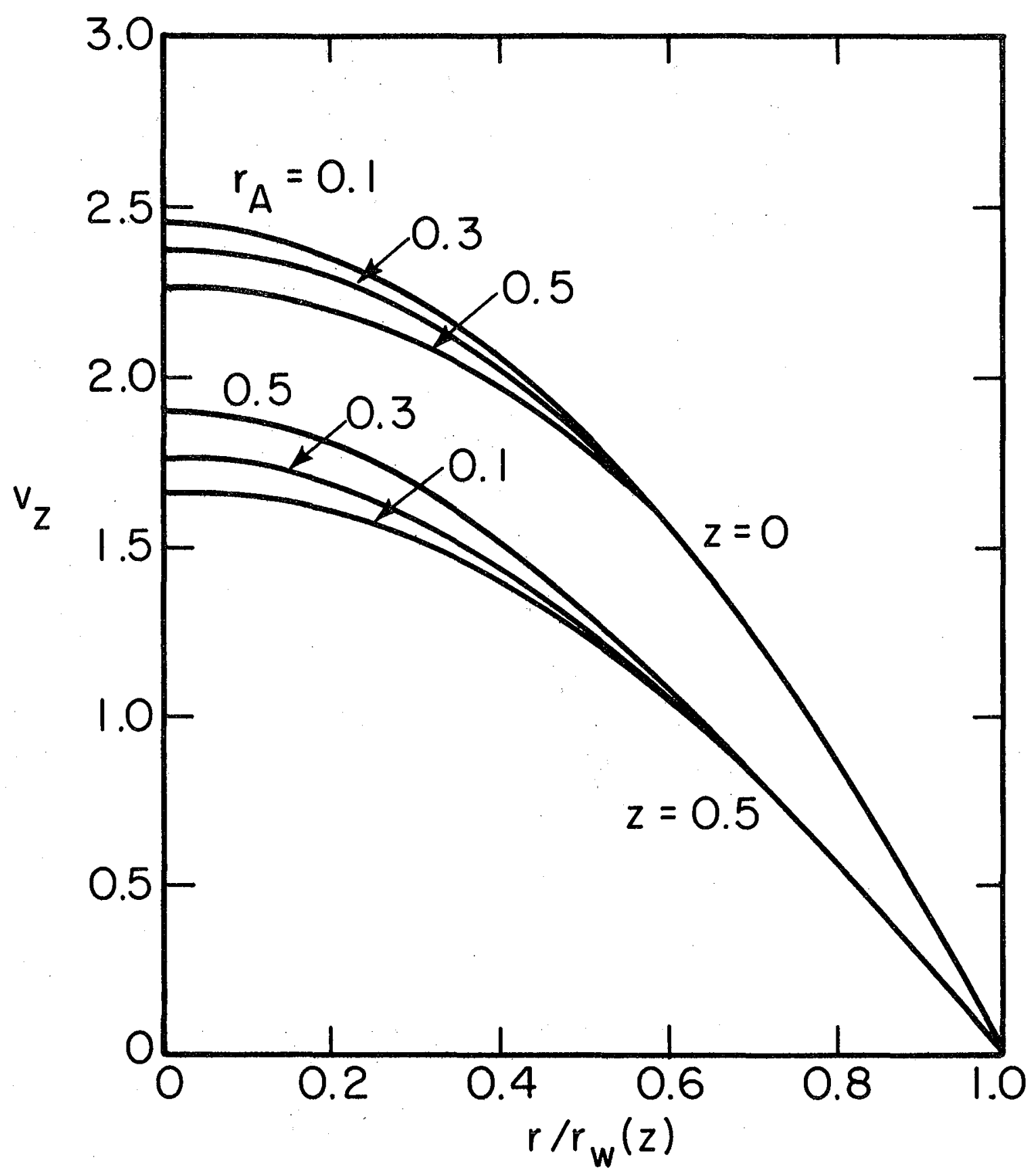

XBL 7811-12887

Figure 3.7 Effect of average tube radius on axial velocity profiles in a sinusoidal PCT for $A / r_{A}=0.1$. 


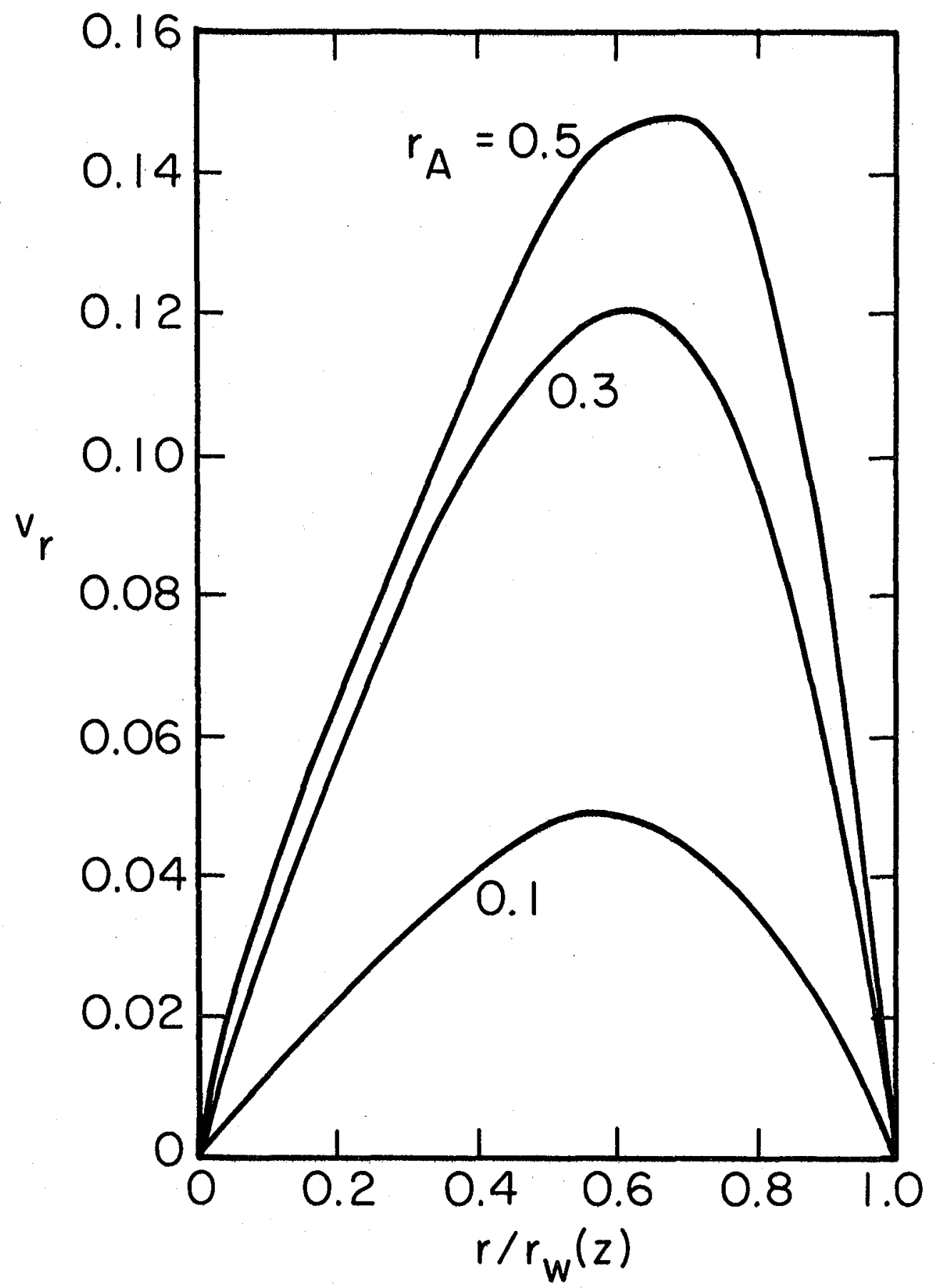

XBL $7811-12888$

Figure 3.8 Effect of average tube radius on radial velocity profiles in a sinusoidal PCT for $\mathrm{A} / \mathrm{r}_{\mathrm{A}}=0.1$ at $\mathrm{z}=0.25$. 
the half period become less pronounced with increasing $\mathrm{r}_{\mathrm{A}}$. This effect is due to the drag induced by the wall. As $r_{A}$ increases the effect of the wall fluctuations become less important to the fluid in the central core of the tube.

The profiles of figures 3.5 thru 3.8 have been nondimensionalized with respect to the average axial velocity at the average tube radius. This normalization procedure illustrates the variation of the profiles from that at the average tube radius. If these profiles are multiplied by $\left(\frac{\mathrm{r}_{\mathrm{W}}(z)}{\mathrm{r}_{\mathrm{A}}}\right)^{2}$, the resulting profiles are then normalized by the average axial velocity at position $z$. Such a calculation shows that the parabolic axial veloctty profile is approached as $\mathrm{r}_{\mathrm{A}}$ becomes smaller. The radial velocity profile is then given by continuity. In the limit of $r_{A} \rightarrow 0$, the Hagen-Poiseuille case is recovered.

Figure 3.9 illustrates the bed friction factor, Reynolds number product of equation 10 as a function of $r_{A}$ and $A / r_{A}$. The product $\mathrm{f}_{\mathrm{B}} \operatorname{Re}_{\mathrm{B}}$ involves the macroscopic bed parameters $\mathrm{L}, \varepsilon$, and $a$. The microscopic PCT parameters $\mathrm{r}_{\mathrm{A}}$ and $\mathrm{A} / \mathrm{r}_{\mathrm{A}}$ can be varied while holding these bed parameters constant. As $\mathrm{A} / \mathrm{r}_{\mathrm{A}}$ increases, the tubes become more narrow at their constrictions. Because of the increased resistance this reduced flow area offers, the bed pressure drop increases with $\mathrm{A} / \mathrm{r}_{\mathrm{A}}$. This effect decreases with larger $\mathrm{r}_{\mathrm{A}}$ since the constriction size at any $\mathrm{A} / \mathrm{r}_{\mathrm{A}}$ increases with $\mathrm{r}_{\mathrm{A}}$.

The relative insensitivity of $f_{B} R e_{B}$ with $r_{A}$ seen in figure 3.9 supports the approximation of assuming the Hagen-Poiseuille pressure gradient, flowrate relationship holds locally for sinusoidal PCT. This 


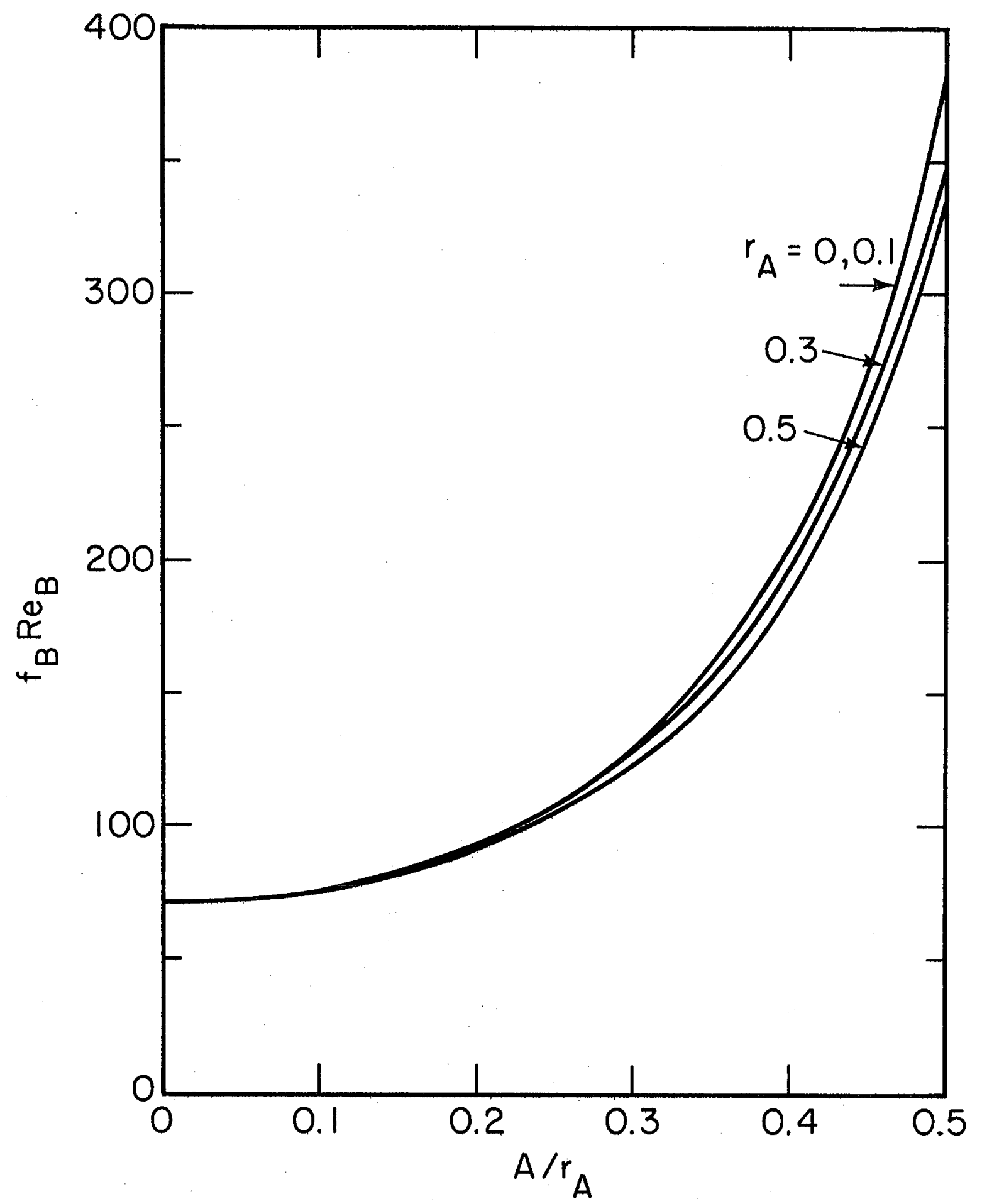

XBL 7811-12889

Figure 3.9 Friction factor, Reynolds number product for a packed bed modeled as an array of sinusoidal PCT. 
approximation was used by Sheffield and Metzner ( 58 ).

The Blake-Kozeny equation as given in Bird et al. ( 59 )

empirically recommends a value of 150 for the product $f_{B} \operatorname{Re}_{B}$.

S $\phi$ rensen and Stewart ( 11 ) have calculated the velocity profiles across a simple cubic packing of uniformly sized spheres. Their pressure-drop results yield a theoretical value of 158 . Figure 3.9 shows that a range of parameters $\left(r_{A}, A / r_{A}\right)$ will give a $f_{B} \operatorname{Re}_{B}$ near these two values. The $A / r_{A}$ ratio which give $f_{B} R_{B}$ a value near 150 seem to be concentrated near 0.33 .

The straight tube capillary model gives the intercept value of 72 on figure 3.9 The usual argument given in explaining the discrepancy between this value and the empirically best fit value of 150 is a tortuosity and shape factor. The PCT model of a packed bed does not resort to these factors. However, another geometrical parameter $\left(\mathrm{A} / \mathrm{r}_{\mathrm{A}}\right)$ has been added.

The mass-transfer analysis presented in this work can be used to calculate the high Péclet number asymptotic Sherwood number for any periodic tube. Only the stream function need be known. Calculated results are presented for the sinusoidal PCT of figure 3.2 in creeping flow. The results are a function of the two dimensionless geometric parameters $r_{A}$ and $A / r_{A}$.

Figure 3.10 presents the first eigenvalue of equation 21 normalized with respect to the first eigenvalue of the straight-tube Graetz problem $\left(\lambda_{G}=0.91419\right)$. This plot can also be interpreted as the ratio of the asymptotic Sherwood number (based on the average radius $r_{A d}$ ) 


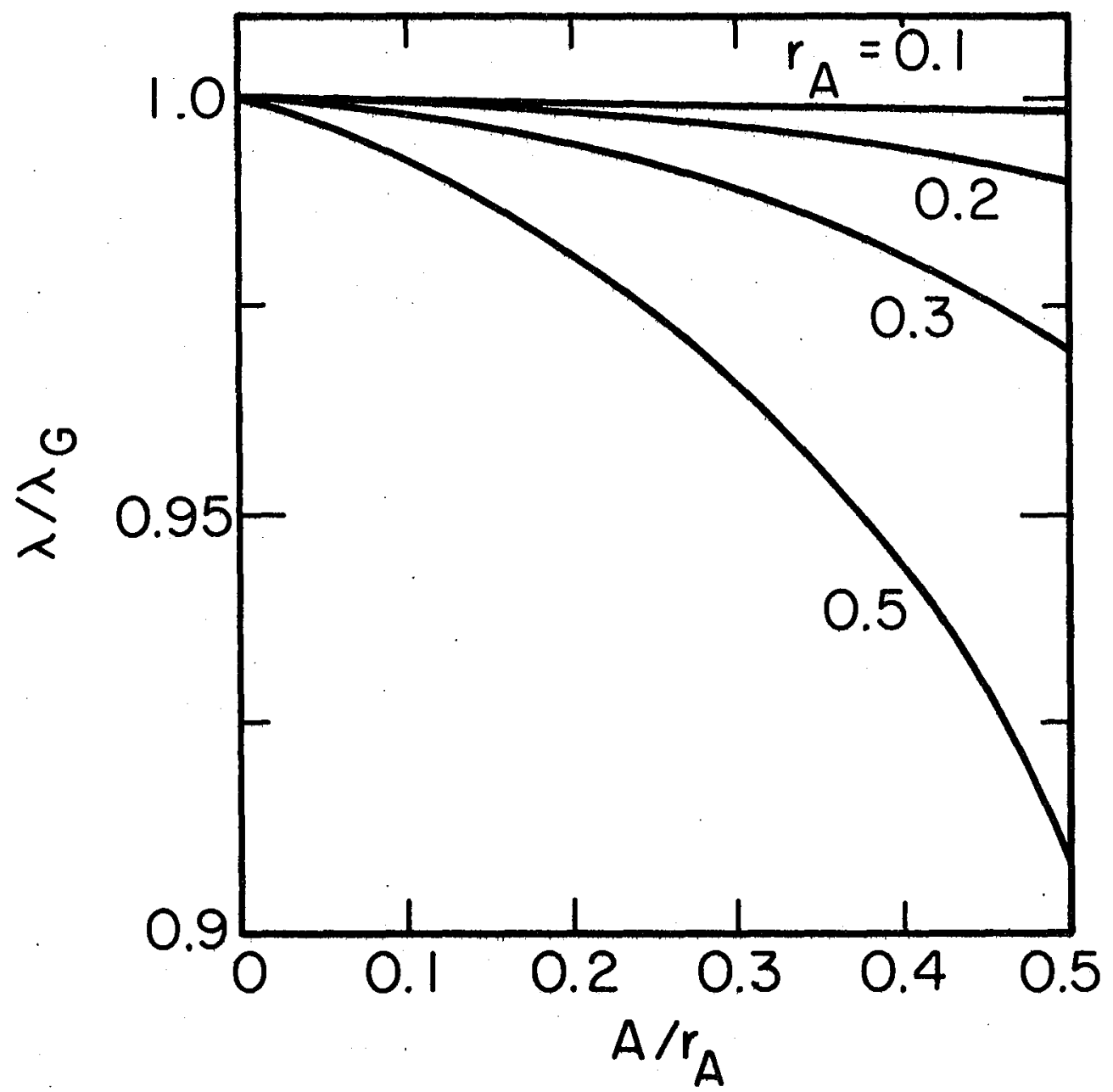

XBL $7811-12890$

Figure 3.10 Eigenvalues for the mass transfer problem in a sinusoidal PCT normalized with respect to the Graetz problem. 
of a sinusoidal PCT to that in a straight tube of radius $r_{\mathrm{Ad}}$.

Figure 3.11 presents the Sherwood number for a packed bed modeled as an array of sinusoidal PCT. The concentration drop across the bed can be written as

$$
\ln c_{F} / c_{L}=S h_{B} \frac{a L}{\varepsilon} / P e_{B}
$$

Figure 3.10 shows a monotonic behavior of the eigenvalues with ${ }_{A}$ and $A / r_{A}$. However, the bed sherwood number shows different trends for small and large $r_{A}$. For small $r_{A}, S h_{B}$ increases with $A / r_{A}$, whereas for larger $r_{A}$ this trend reverses itself. This effect is caused by the geometrical term in equation 26 .

The quantity $2 \varepsilon / a$ in equation 26 is the standard definition for the equivalent radius of the bed. This defines the bed in terms of a straight cylinder network of radius $r_{e q, d}$ having the same surface area to empty volume ratio. The quantity $\mathrm{r}_{\mathrm{Ad}}^{2}\left[1+(1 / 2)\left(\mathrm{A} / \mathrm{r}_{\mathrm{A}}\right)^{2}\right]$ in the denominator of equation 26 defines another equivalent radius $\mathrm{r}_{\mathrm{Vd}}^{2}$. This is the volumetric average radius for a sinusoidal PCT. For long skinny PCT (small $r_{A}$ ), the ratio $\left(r_{e q} / r_{v}\right)^{2}$ is greater than one and increases with $\mathrm{A} / \mathrm{r}_{\mathrm{A}}$. Thus for a bed composed of these tubes, the Sherwood number increases as $\mathrm{A} / \mathrm{r}_{\mathrm{A}}$ is increased. However, as $\mathrm{r}_{\mathrm{A}}$ becomes larger, the ratio $\left(\mathrm{r}_{\mathrm{eq}} / \mathrm{r}_{\mathrm{v}}\right)^{2}$ becomes less than one and $\mathrm{Sh}_{\mathrm{B}}$ decreases with $\mathrm{A} / \mathrm{r}_{\mathrm{A}}$.

For most beds, ${ }^{r_{A}}$ will be bounded approximately by $0.3<r_{A}<0.5$ while the $A / r_{A}$ ratio will be in the range $0.2<A / r_{A}<0.5$, perhaps 


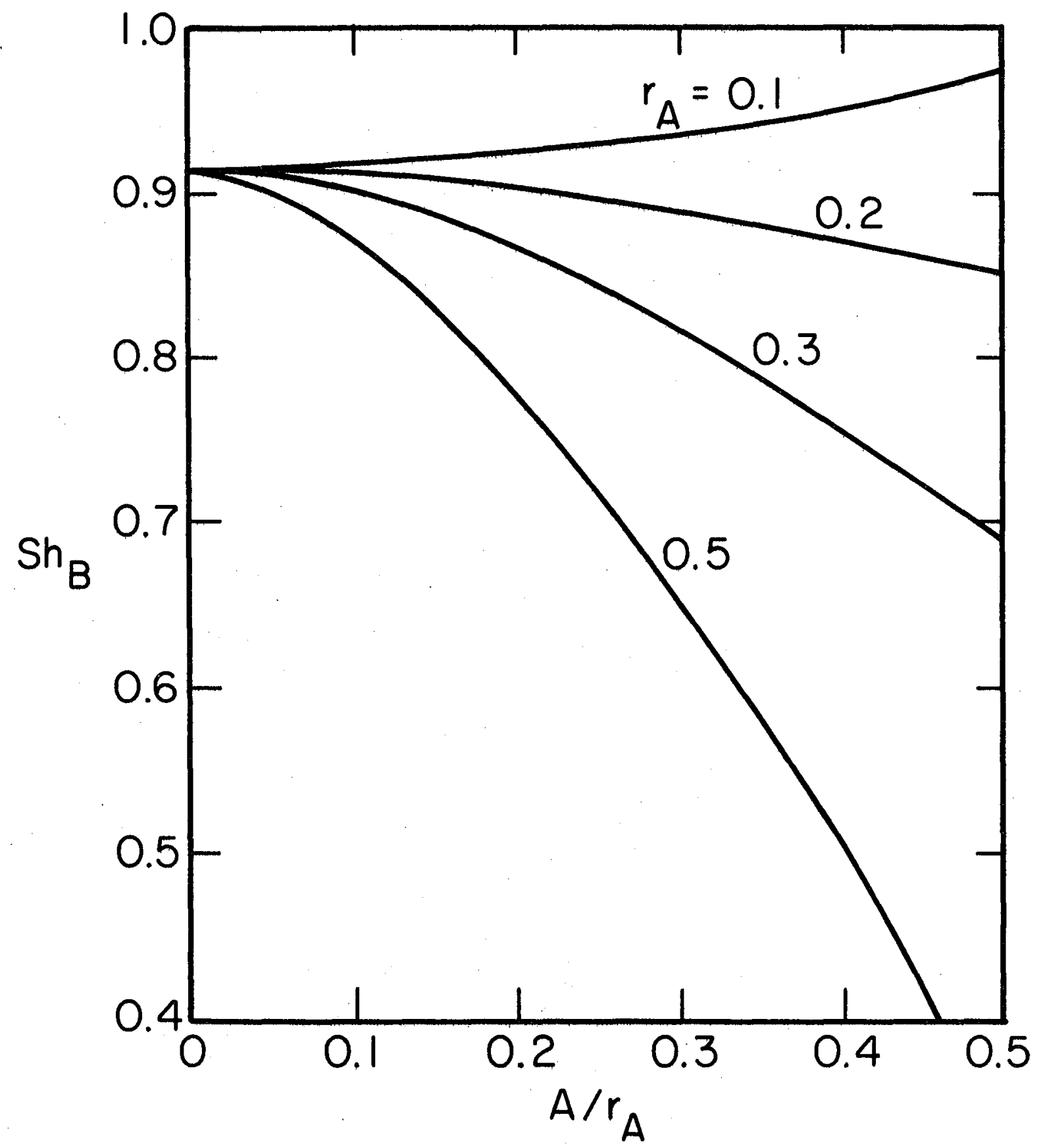

XBL 7811-12891

Figure 3.11 Asymptotic Sherwood number for a packed bed modeled as an array of sinusoidal PCT. 
close to 0.33 . Payatakes et al. report these parameters for a randomly packed bed of glass spheres as $\mathrm{r}_{\mathrm{A}}=0.3, \mathrm{~A} / \mathrm{r}_{\mathrm{A}}=0.36$, and for $a$ bed of sand as $r_{A}=0.31, A / r_{A}=0.41$.

Sфrensen and Stewart ( 12 ) have calculated the asymptotic value of the Sherwood number in a simple cubic packed bed of uniformly sized spheres. Their results yield $\mathrm{Sh}_{\mathrm{B}}=0.619$. This information can be used in conjunction with the friction factor, Reynolds number product calculated by these same authors. This suggests that the PCT parameters for a simple cubic packing of spheres are $r_{A} \approx 0.5$ and ${ }^{A} / r_{A} \approx 0.33$. We expect this ${ }{ }_{A}$ value to be an upper limit for uniform spheres since the simple cubic packing has the highest porosity of all sphere packing configurations.

No experimental packed bed heat or mass-transfer correlations are known to the authors which demonstrate a transfer rate independent of velocity. Three factors can mask this asymptote. 1) At very low velocities axial dispersion may become important. 2) At high flowrates turbulence becomes important. 3) At the intermediate flowrates the entire bed may be in the entry region (smal1 aL). However, the asymptotic Sherwood number gives a conservative estimate useful for design purposes.

The solution to the creeping flow equations exhibited separation flow for some values of the geometry parameters. (See chapter 6 for the range of these parameters.) These separation zones were neglected in this analysis for simplicity sake. Consequent1y, the Sherwood number is underestimated in this parameter range. 


\author{
Chapter 4 \\ Low Péclet Number Behavior of the Transfer Rate \\ in Packed Beds
}

\title{
$\underline{\text { Abstract }}$
}

The asymptotic behavior of the mass-transfer coefficient in a packed bed reactor at low Péclet numbers is dependent upon how the coefficient is defined. A singular perturbation approach coupled with heuristic arguments is used to demonstrate that the film masstransfer coefficient in deep beds approaches a constant value as the Péclet number decreases. The film coefficient is utilized in the one-dimensional model of a bed as a sink term in the governing equation.

The volumetric, or effective, mass-transfer coefficient, which relates the overall reactant conversion to a logarithmic mean concentration driving force, decreases linearly with the Péclet number as the Péclet number approaches zero. The distinction between the two coefficients is important in the low Péclet number region. Analogous results apply to heat transfer. Reported experimental data support these predicted trends. 


\section{Introduction}

The behavior of the particle-to-fluid (or vice-versa) heat or mass transfer rate in packed beds at low Péclet numbers has been a source of confusion in the chemical engineering literature. The question is, "Does the transfer rate approach a steady value as the Péclet number decreases, or does it continually decrease with the Péclet number?" This can be rephrased by asking whether the Sherwood number reaches a constant value or decreases as the Péclet number is lowered. We sha11 demonstrate in this paper that both trends are possible depending upon how the Sherwood number is defined.

The experimental determination of transfer coefficients at low Péclet numbers is vexing. The fluid leaving the bed is very near its saturation value in the transferred quantity. This creates a large uncertainty in the driving force at the exit of the bed which is used in defining the effective transfer coefficient. Free convection may also become an important effect. To overcome these difficulties, various workers have used diluted beds, transient, and frequency response methods to determine more accurately the lowPéclet-number behavior.

Since the Schmidt and Prandt1 numbers for 1iquids are quite large $\left[0\left(10^{3}\right)\right]$, most low Péclet number data are found in gaseous systems. Furthermore, most workers have varied the Reynolds number only. The free convection effects should be minimized in the gaseous systems.

The quantity of data for low Péclet numbers is understandably small. Table 4.1 is a compilation (with no claim to completeness) of 
Table 4.1 Compilation of works which have reported heat or mass transfer data in packed beds for a particle Péclet number less than $30 .^{\dagger}$.

\begin{tabular}{|c|c|c|c|c|}
\hline Investigators & $\begin{array}{l}\text { Lowest Reynolds } \\
\text { number reported }\end{array}$ & $\begin{array}{l}\text { Lowest Schmidt or } \\
\text { Prandt1 number } \\
\text { reported }\end{array}$ & $\begin{array}{l}\text { Lowest Péclet } \\
\text { number }\end{array}$ & $\begin{array}{l}\text { Heat }(\mathrm{H}) \\
\text { or } \ldots \\
\text { Mass }(\mathrm{M})\end{array}$ \\
\hline $\begin{array}{l}\text { Resnick and White } \\
\text { (1949) }\end{array}$ & 0.62 & 4.0 & 2.5 & M \\
\hline $\begin{array}{l}\text { Eichorn and White } \\
\text { (1952) }\end{array}$ & 1 & $0.7 *$ & 0.7 & M \\
\hline$\underset{(1953)}{\operatorname{Dryden}}$ et & 0.0125 & 814 & 10.2 & M \\
\hline $\begin{array}{l}\text { Bar-Ilan and Resnick } \\
\text { (1957) }\end{array}$ & 0.2 & 2.6 & 0.5 & M \\
\hline$\underset{(1968)}{\text { Littman et }}$ al. & 1.9 & $0.7 *$ & 1.3 & H \\
\hline $\begin{array}{l}\text { Petrovic and Thodos } \\
\text { (1968) }\end{array}$ & 3 & 0.6 & 1.8 & M \\
\hline $\begin{array}{l}\text { Kato, }, \frac{\text { et }}{\text { a1 }} . \\
\quad(1970)\end{array}$ & 0.1 & 2.6 & 0.3 & M \\
\hline $\begin{array}{l}\text { Gliddon and Cranefield } \\
\text { (1970) }\end{array}$ & 23.8 & 0.7 & 16.4 & H \\
\hline $\begin{array}{l}\text { Karabelas et al. } \\
(1971)\end{array}$ & 0.01 & 1490 & 15 & M \\
\hline $\begin{array}{l}\text { Gunn and De Souza } \\
\text { (1974) }\end{array}$ & 1.5 & $0.7 *$ & 1.1 & $\mathrm{H}$ \\
\hline$\underset{(1975)}{\text { Miyaucht et }}$ al. & 0.02 & 510 & 10 & $M=$ \\
\hline $\begin{array}{l}\text { Ne1son and Galloway } \\
\text { (1975) }\end{array}$ & 1 & $0.7 *$ & 0.7 & $\mathrm{H}^{-}$ \\
\hline$\underset{(1976 a)}{\text { Miyauchi }}$ et al. & $?$ & $?$ & 2 & $M$ \\
\hline $\begin{array}{l}\text { Appe1 and Newman } \\
\text { (1976) }\end{array}$ & 0.00806 & 1440 & 11.6 & M \\
\hline
\end{tabular}


those publications which list packed-bed transfer data for Péclet numbers less than 30 . Both heat and mass transfer results are included. The data scatter as the Péclet number decreases, and there is no absolute agreement between different authors. (This is true, to an extent, no matter what the Péclet number range.) There is, however, a definite trend in the Sherwood number (or equivalently, the Colburn j factor) with the Péclet number reported by the authors of Table 4.1. The Sherwood number based on an averaged inlet and outlet concentration (or temperature) driving force across the bed $(47,67,66,70,49,17$, 69,65 ) seems to decrease with the Péclet number. However, the film Sherwood number $(72,68,73,74,75$ ) seems to reach a constant as the Péclet number decreases. The film Sherwood number is calculated by parameter fitting the experimental data to the solution of the governing one-dimensional convective diffusion equation.

A concise definition of these two different Sherwood numbers follows. We shall demonstrate that the distinction between these two numbers is small for large Péclet numbers but becomes important as the Pêclet number approaches zero.

\section{Definitions of the Mass-Transfer Coefficient}

To be specific in our discussion, we shall speak in terms of the mass-transfer problem in a nonconsolidated packed bed. The results are applicable to the heat-transfer problem by the usual analogies. We sha1l also limit our discussion to a single reaction at the particle surfaces. The rate of reaction is controlled by the reactant mass transfer from the fluid to the particle surface. 
A design engineer is interested in the performance capabilities of a packed bed reactor. Given a certain feed concentration of reactant, he is interested in the overall reactant conversion. This can be correlated in terms of the bed's effective mass-transfer coefficient $k_{m}$.

$$
\mathrm{v}\left[\mathrm{c}_{\mathrm{F}}-\mathrm{c}_{\mathrm{L}}\right]=\mathrm{ak} \mathrm{c}_{\mathrm{m}} \mathrm{L} \Delta \mathrm{c}_{\text {In }}
$$

The concentrations $c_{F}$ and $c_{L}$ are the far upstream and downstream reactant concentrations. Equation 1 relates the conversion to a logarithmic mean driving force. (Some workers have chosen different driving forces. Bird et al. (59) give a lucid discussion of the possibilities.) For the limiting reactant condition considered here, equation 1 reduces to

$$
\frac{c_{L}}{c_{F}}=\exp \left(-\frac{S h_{B}}{P e_{B}} \frac{a L}{\varepsilon}\right)
$$

where the bed Sherwood and Péclet numbers have been introduced. It is also possible to define a film coefficient $k_{f}$. This coefficient relates the local concentration driving force in the bed to the local reaction rate. It is assumed for a given flowrate that $k_{f}$ does not vary throughout the bed. The term $\left(k_{f} c\right)$ appears as a sink term in the one-dimensional equation governing the concentration profile in the bed: 


$$
E \frac{d^{2} c}{d z^{2}}-v \frac{d c}{d z}=a k_{f} c
$$

The dispersion coefficient $E$ is a function of the flow regime. In the limit of low Péclet numbers, $E$ is dominated by molecular diffusion and reduces to

$$
E=\varepsilon \frac{D_{0}}{\tau}
$$

where $\tau$ is a tortuosity factor (43).

It might seem that $k_{f}$ is a more fundamental quantity than $k_{m}$ (or vice-versa, depending upon one's point of view). This is not so, however, since both $k_{m}$ and $k_{f}$ are essentially defined quantities. These two coefficients are related. This can be seen by solving equation 3 with appropriate boundary conditions to find the concentration field across the bed. After solving this expression for $c_{L} / c_{F}$ and setting this result equal to equation 2 , one obtains ( 34 )

$$
k_{m}=\frac{k_{f}}{B}+\frac{v}{a L} \ln \left\{\frac{B+\frac{D^{\prime}}{B^{2}}(1-B) \exp \left[-\alpha L\left(\frac{1}{B}+\frac{B}{D^{\prime}}\right)\right]}{1+D^{\prime} / B^{2}}\right\}
$$

where

$$
\begin{aligned}
\alpha & =a k_{f} / v \\
D^{\prime} & =a k_{f} E / v^{2} \\
B & =\frac{1+\sqrt{1+4 D^{\prime}}}{2} .
\end{aligned}
$$


The boundary conditions used to solve equation 3 were those given by Wehner and Wilhelm's analysis (38).

$$
\begin{array}{lll}
\mathrm{vc}_{F}=-\mathrm{E} \frac{\mathrm{dc}}{\mathrm{d} z}+\mathrm{vc} & \text { at } & z=0 \\
\frac{\mathrm{dc}}{\mathrm{d} z}=0 & \text { at } & z=L
\end{array}
$$

Equation 4 shows how the experimentally accessible, and design useful, $k_{m}$ can be corrected to give the film coefficient $k_{f}$. For purely pedagogical purposes, assume $k_{f}$ is given by the WilsonGeankopolis correlation (52). Figure 4.1 (34) illustrates how $\mathrm{k}_{\mathrm{m}}$ would then vary with the Péclet number. At large Péclet numbers, the distinction between the two coefficients vanishes. However, as $\mathrm{Pe}_{\mathrm{B}} \rightarrow 0$, the difference between the two becomes important. It should perhaps be emphasized that the calculation of $k_{f}$ from $k_{m}$ requires a value of $\mathrm{E}$, with which there must be associated some uncertainty.

\section{Calculation of $\mathrm{k}_{\mathrm{m}}$}

It would not be necessary to use the one-dimensional model for a bed and its associated film coefficient if we could describe the void volume in the bed analytically. For it would then be possible (in principle) to solve the hydrodynamics and the convectivediffusion within the voids to calculate directly the overall conversion. This is an overwhelming task. The voids in a bed defy 


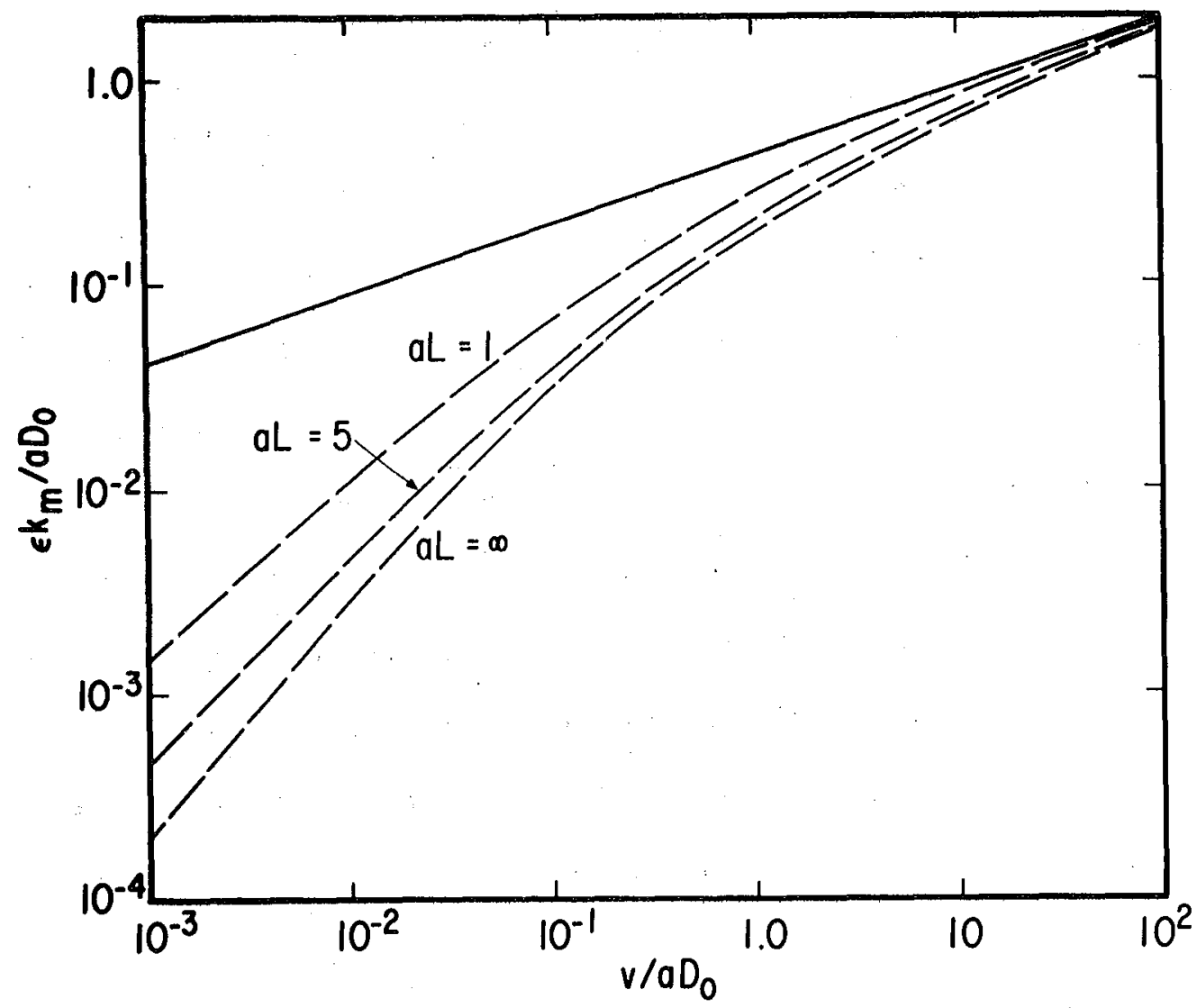

XBL 7811-12298

Figure 4.1 Influence of axial dispersion on the effective masstransfer coefficient $\mathrm{k}_{\mathrm{m}}$. The film mass-transfer coefficient $k_{f}$ is assumed to be given by the WilsonGeankopolis correlation as shown in the upper curve. Because of axial dispersion, $k_{m}$ lies below $k_{f}$, and the effect becomes large at low Péclet numbers. In preparing the graph, the porosity was taken to be $\varepsilon=0.3$ and the tortuosity factor $\tau$ was assumed to be 1 . The convective contribution to the dispersion coefficient was given by equation 3.9 . 
an analytic expression except in a simple instance $(11,12)$. This approach, however, can be used to indicate the asymptotic behavior of the fluid-to-particle transfer rate at low Peclet numbers. We shall see that a detailed knowledge of the void volume function is not necessary to establish the behavior in this limit.

Assuming a known bed geometry, we sha11 demonstrate how $k_{m}$ (not $\mathrm{k}_{\mathrm{f}}$ ) behaves asymptotically as $\mathrm{Pe}_{\mathrm{B}} \rightarrow 0$. Equation 4 with the proper form of the dispersion coefficient can then be used to indicate the behavior of $k_{f}$.

Wehner and Wilhelm (38) showed how to analyze correctly the behavior of a one-dimensional model for a packed bed reactor. Their analysis can be extended to the actual three-dimensional structure of the bed. As shown in figure 4.2, the bed consists of three regions. Region II of length $L$ is the reactive section of the bed. Regions I and III extend in the dimensionless streamwise coordinate $x_{1}$ to $-\infty$ and $+\infty$, respectively. They are filled with an inert packing. These are the "calming sections" used in experimental apparatus. A cross section normal to the streamwise direction is finite in extent.

The position of the particles' surface is assumed to be known as a function of the streamwise coordinate. Designate this function as $W_{a}\left(x_{1}\right)$ for the reactive particles and $W_{1}\left(x_{1}\right)$ for all other inactive surfaces.

Neglecting free convection effects, the concentration field in the voids will satisfy the dimensionless convective-diffusion equation. 


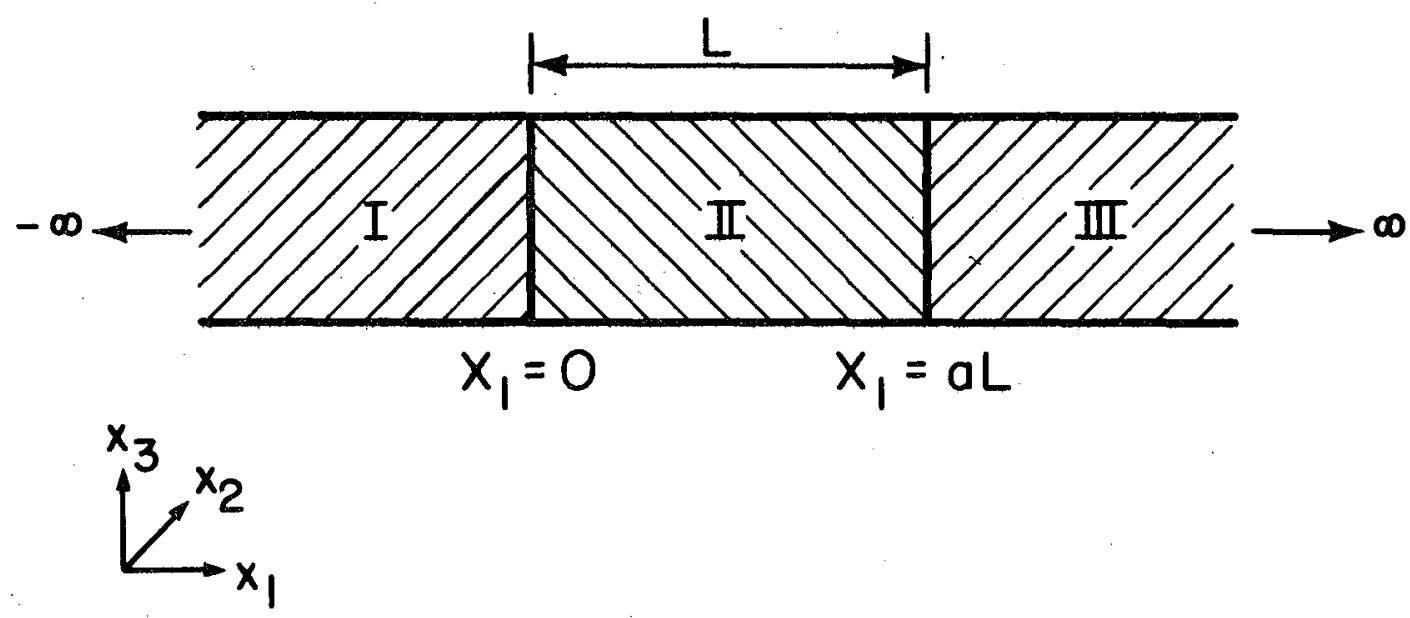

XBL 7811-12299

Figure 4.2 Schematic of a packed bed with fore and aft "calming" sections. 


$$
\overrightarrow{\mathrm{v}} \cdot \nabla \mathrm{c} / \mathrm{c}_{\mathrm{F}}=\frac{1}{\mathrm{Pe}} \nabla_{\mathrm{B}}^{2} \mathrm{c} / \mathrm{c}_{\mathrm{F}}
$$

Equation 6 is subject to the following boundary conditions.

$$
\begin{aligned}
& \text { i) } x_{1} \rightarrow-\infty \quad \frac{c}{c_{F}}=1 \\
& \text { ii) }-\infty<x_{1}<\infty \quad \frac{\partial\left(\frac{c}{c_{F}}\right)}{\partial n}=0 \text { on } w_{i}\left(x_{1}\right) \\
& \text { iii) } 0 \leq x_{1} \leq a L \quad \frac{c}{c_{F}}=0 \text { on } W_{a}\left(x_{1}\right) \\
& \text { iv) } x_{1} \rightarrow \infty
\end{aligned}
$$

These boundary conditions yield a wel1-posed problem when applied to equation 6 in the void volume.

The detailed solution to the convective diffusion equation directly yields the concentration exiting the reactor and thus, through equation 2, the bed Sherwood number. Dimensional analysis indicates that the Sherwood number will be, in general, a function of the velocity field, the parameters $\mathrm{Pe}_{\mathrm{B}}$, $\mathrm{aL}$, and the geometric functions $W_{i}\left(x_{1}\right)$ and $W_{a}\left(x_{1}\right)$. In the following, we sha1l develop the explicit functional dependence on $\mathrm{Pe}_{\mathrm{B}}$ and $\mathrm{aL}$ at low Péclet numbers and for deep beds. 
We want to consider the solution to equations 6 and 7 in the limit of zero Péclet number. This limiting process is singular in nature. There are regions in the bed where diffusion does not dominate the reactant transport and convection becomes important. These regions will be located in the upstream and downstream "calming" sections. There are precedents for this expected behavior in the literature. Acrivos and Taylor (76) analyzed a single reactive sphere in Stokes flow as the particle Péclet number approaches zero. They have shown that, near the sphere, diffusion controls the mass-transfer rate to the surface, but convection also becomes important far from the surface. Lea1 (77) has extended this analysis to a sphere in a simple shear field. Such work suggests that applying the asymptotic limit of $\mathrm{Pe}_{\mathrm{B}} \rightarrow 0$ will require a singular perturbation approach. To formulate properly a singular perturbation problem for the concentration field within the voids of the bed, it is necessary to delineate the regions where diffusive and/or convective transport are controlling. Appropriate transformation variables must also be defined for each region. The equations governing the concentration 
and their boundary conditions should then be independent of the Péclet number in the regions.

In the reactor section (II) and a region on the "calming" side of the boundaries I-II and II-III, the inner solution applies. In the inner expansion, diffusion dominates the reactant transport. Far away from the reactor section, in the upstream (I) and downstream (III) regions, the diffusive and convective flux of reactant become equally important. Thus, there is an upstream and downstream outer region to the expansion.

Consideration of the Wehner-Wilhelm solution for a one-dimensional bed leads to the appropriate transformed concentration and coordinates in each region.

$$
\begin{aligned}
& \tilde{\theta}^{I}=c / c_{F} \\
& \tilde{x}_{j}=x_{j} \frac{P e_{B}}{\varepsilon}(j=1,2,3) \\
& \text { upstream } \\
& \text { outer } \\
& \text { variables } \\
& \bar{\theta}=\frac{c \varepsilon}{c_{F} e_{B}} \\
& \bar{x}_{j}=x_{j} \quad(j=1,2,3) \\
& \text { inner } \\
& \text { variables } \\
& \tilde{\theta}^{\mathrm{III}}=\frac{c \varepsilon}{c_{F} \mathrm{Pe}_{\mathrm{B}}}
\end{aligned}
$$

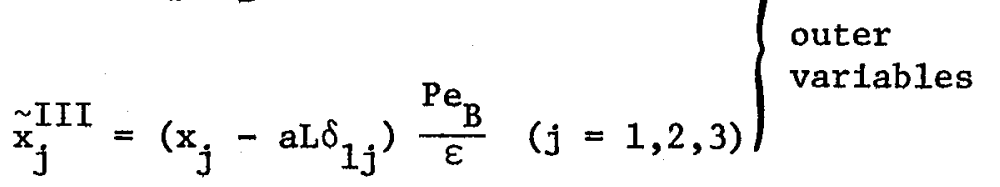


The coordinates $\left(x_{1}, x_{2}, x_{3}\right)$ form an orthogonal system with its origin at the geometric center of the entrance to section II. Coordinate $x_{1}$ is in the streamwise direction. The tilde $(\sim)$ refers to the outer region variables, and the bar $(-)$ refers to inner region variables. The transformed concentrations have been scaled to be of $0(1)$ in each region.

In the upstream and downstream outer region expansion, further simplifications are possible. Far from the perturbing effect of the reactor section, the concentration field will be approaching a constant value at each cross section of the bed, that is, the variation in the axial direction is small over a distance comparable to the size of a particle. Thus the one-dimensional (streamwise) description will suffice as a first-order approximation. The geometry is a second-order effect there. It is accounted for by the tortuosity factor in the effective diffusion coefficient. In the inner region of the expansion, however, the exact placement of the particles is important, and no geometrical simplifications can be made here.

By the above reasoning, the first-order solution in each region is governed by the following equations.

$$
\begin{gathered}
\frac{d^{2} \tilde{\theta}_{o}^{I}}{d\left(\tilde{x}_{1}^{I}\right)^{2}}-\frac{d \tilde{\theta}_{o}^{I}}{d\left(\tilde{x}_{1}^{I}\right)}=0 \\
\nabla^{2} \bar{\theta}_{o}=0
\end{gathered}
$$




$$
\frac{d^{2} \tilde{\theta}_{0}^{I I I}}{d\left(\tilde{x}_{1}^{I I I}\right)^{2}}-\frac{d \tilde{\theta}_{0}^{I I I}}{d\left(\tilde{x}_{1}^{I I I}\right)}=0
$$

Equation 8 is subject to the boundary condition $7(1)$ and equation 10 to 7 (iv). Equation 9 is subject to equations 7 (ii), 7(iij.), and the matching condition imposed by merging the outer limit of the inner solution to the inner limit of the outer solution. This is carried out in the upstream and downstream regions. This matching results in the following additional boundary conditions for equation 9.

$$
\begin{aligned}
& \frac{\partial \bar{\theta}_{0}}{\partial \bar{x}_{1}}=-1 \text { as } \bar{x}_{1} \rightarrow-\infty \\
& \frac{\partial \bar{\theta}_{0}}{\partial \bar{x}_{1}}=0 \text { as } \bar{x}_{1} \rightarrow \infty .
\end{aligned}
$$

The first order governing equations and their necessary boundary and matching conditions have been outlined above. The solution to this system of equations then generates the first-order approximation to the concentration of the reactant leaving the bed. This result can be utillzed in equation 2 to write,

$$
\frac{\varepsilon \mathrm{k}_{\mathrm{m}}}{\mathrm{aD}_{\mathrm{o}}}=\frac{\varepsilon}{\mathrm{aL}} \frac{\mathrm{v}}{\mathrm{aD_{ \textrm {o } }}}\left[\ln 1 / \theta_{\mathrm{L}}-\ln \frac{\mathrm{v}}{\varepsilon \mathrm{aD}}\right]
$$


where $\theta_{L}$ is ${\frac{1}{x_{1} \rightarrow \infty}}_{0} \bar{\theta}_{0}$. Equation 12 has been rigorous1y derived through a singular perturbation approach. The bed Sherwood number for low Péclet numbers can be calculated once $\theta_{L}$ is known. This quantity will depend upon the detailed geometry functions $W_{1}$ and $\mathrm{W}_{\mathrm{a}}$, and the parameter $\mathrm{aL}$, as can be seen by examining equations 8 through 10 and their associated boundary conditions.

Equation 12 may appear to be a rearrangement of equation 1 , and indeed it can be generated from equation 1 through algebraic manipulations. However, equation 12 yields a priori predictions of the Sherwood number after $\theta_{L}$ is determined from the perturbation problem as outlined. In particular, $\theta_{L}$ is shown to be independent of the Péclet number at low Péclet numbers, and the dependence on aL can be elucidated to some extent as discussed below.

Deep bed behavior of $\mathrm{k}_{\mathrm{m}}$

For straight tubes with an insulated upstream wal1 $(z<0)$ and an active downstream wall $(z \geq 0)$, the local mass-transfer rate depends upon the axial position, but the local mass-transfer coefficient approaches an asymptotic value in the downstream region. The length scale in which this asymptotic value is approached depends upon the Péclet number. This region is usually designated the masstransfer entry region. Levich (78) has shown for high Péclet numbers that the entry region is $O(P e R)$, where $R$ is the tube radius. In the low Péclet number regime, Michelsen and Villadsen (79) have shown that the entry region is $O(R)$, a result which is substantiated by 
the calculations of S $\phi$ rensen and Stewart (10) and Michelsen and Villadsen (79).

From these results one concludes that the entry region for a packed bed should be in the order of a particle diameter for low Péclet numbers, and a deep bed thus will be one for which $L>d_{p}$. In this limit, most of the bed will be in the we11-developed masstransfer region, where the fractional decrease of reactant for any incremental streamwise length should be independent of the position of the incremental length. Thus, for deep beds, the dependence of $\theta_{\mathrm{L}}$ on aL can be expressed as

$$
\theta_{\mathrm{L}}=\alpha_{2} \quad \mathrm{e}^{-\alpha_{1} \mathrm{aL} / \varepsilon},
$$

where $\alpha_{1}$ and $\alpha_{2}$ are independent of aL (as well as Pe) and are dependent upon the detailed geometric functions $W_{1}$ and $W_{a}$. The deep bed, low $\mathrm{Pe}_{\mathrm{B}}$ Sherwood number from equation 12 can then be written as

$$
\frac{\varepsilon k_{m}}{a_{0}}=\frac{v}{a_{o}}\left[\alpha_{1},-\frac{\varepsilon}{a L} \ln \frac{\alpha_{2} v}{\varepsilon a D_{o}}\right] \text {. }
$$

Since the entry region is small at low Péclet numbers, this result should be applicable to many beds of practical importance. Equation 14 was first stated by Sørensen and Stewart (10), but they did not demonstrate how they derived this result. 
Equation 14 shows that for large values of aL, where the second term in the brackets is negligible compared to the first, $k_{m}$ becomes linearly proportional to the velocity. (Note that in figure 4.1 the curve for $\mathrm{aL}=5$ is fairly close to that for $\mathrm{aL}=\infty_{\text {.) }}$ After applying the large aL limit and the low Péclet number 1imit; one can conclude from equation 4 that the film coefficient of the one-dimensional model for the same bed must approach a constant as

$$
\frac{\varepsilon k_{f}}{a_{o}}=\alpha_{1}^{2} / \tau
$$

Equations 14 and 15 are the main result of this chapter. It is worth noting that equation 14 could be derived from equation 4 in a simpler manner by a priori postulating that $\mathrm{k}_{\mathrm{f}}$ becomes independent of $v$ for low Péclet numbers. However, this of course would not shed any conclusive light on the real behavior of $k_{f}$.

The void volume approach outlined above cannot be carried further for the general case without specifying the geometry. A packed bed can be considered on the microscopic scale as a statistically perlodic structure (29). In order to introduce a predictive capability into the present method, one can solve equation 9 in the well-developed mass-transfer region as an eigenvalue problem. This yields the fractiona1 decrease of reactant for each period and hence the value of $\alpha_{1}$ in equation 13. For significant values of aL, this also yields the dominant part of $k_{m}$ according to equation 14 and $k_{f}$ according to equation 15. 
Discussion

The two types of mass-transfer coefficients described in this work have been reported in the literature. Miyauchi and his coauthors $(80,73,74,75,83)$ conclude from the analysis of their data that the film coefficient reaches a constant as the Pêclet number decreases. Gunn and Souza (72) and Littman et al. (68) also reach the same conclusion about their data. However, their results exhibit more scatter than those of Miyauchi. On the other hand, from considering those workers' results $(47,67,66,70,49,17,69,65$ ) who calculated an effective transfer coefficient as in equation 1 (or its possible equivalent forms), one could conclude that the effective transfer coefficient decreases with decreasing Pêclet number.

There are clearly different experimental trends in these two coefficients. Our analysis suggest that the effective Sherwood number becomes linearly proportional to the Péclet number as $\mathrm{Pe} \rightarrow 0$. This is true for any geometric arrangement of the voids in the bed. This implies through equation 4 that the film coefficient approaches a constant in the same limit. These prediced trends agree with the available experimenta1 data.

We have not been concerned in this paper with presenting numerical predictions for the film or effective mass-transfer coefficients at low Péclet numbers. However, several comments on some previous theoretical work along this line are in order. To predict the transfer coefficients in a bed, it is necessary to introduce a microscopic model for the structure of the bed. The 
free-surface cell model developed by Happel (32) or an analog of this as introduced by Neal and Nader (13) has been used by various authors. One can calculate a film mass-transfer coefficient by solving the hydrodynamics and the convective-diffusion equation within the cell, both subject to appropriate boundary conditions. This film coefficient will depend upon the flow conditions in the cell and the Schmidt number. As our analysis suggests, only in the high Péclet number region is this film coefficient equal to the effective coefficient $\mathrm{k}_{\mathrm{m}}$. Pfeffer (18), Pfeffer and Happe1 (15), E1-Kaissy and Homsy (19) and Tardos et al. (21. ) have performed such calculations for high Péclet numbers within the free-surface cell model framework.

At low Péclet numbers, the uniform concentration boundary condition imposed on the outer free surface of the cell has been criticized $(81,17,10)$. Nelson and Galloway (17) attempted to remedy this ficticious sink boundary condition. They imposed a zero-radial gradient condition on the concentration at the outer free surface. They combined surface-renewal and boundary-layer arguments to arrive at a film coefficient linearly proportional to the Reynolds number and to the Schmidt number raised to the two-thirds power. At low Reynolds numbers the applicability of surface-renewal theory and boundary-layer theory is questionable. Also, the distinction between the film and effective mass-transfer coefficients was not recognized.

Kunii and Suzuki (82) have realized the difference between the two coefficients at low Péclet numbers. They have presented 
a channeling model to calculate $\mathrm{k}_{\mathrm{m}}$. They predict a linear dependence on the Péclet number. Their model involves an empirical factor which varies over two orders of magnitude to fit their data analysis.

Sфrensen and Stewart $(11,12)$ have numerically calculated the creeping flow velocity profiles and solved the convective-diffusion equation for a limiting reactant in a simple cubic packed bed of uniform sized spheres. These calculations are a great aid in the understanding of processes in an actual bed. Their results indicate that the effective coefficient $k_{m}$ varies linearly with the Péclet number as $\mathrm{Pe}_{\mathrm{B}} \rightarrow 0$ in accord with equation 14. Their results can be used to calculated $\alpha_{1}$ in equation 14, and hence, through equation 15, $k_{f}$. The dimensionless film coefficient for a deep bed of simplecubic packed spheres $(\varepsilon \sim 0.48)$ is found to be

$$
\frac{d_{p} k_{f}}{D_{o}}=7.1
$$

where a tortuosity factor of $\tau=\sqrt{2}$ has been assumed. This should be compared to the experimental values of 12.5 determined by Miyauchi et al. (83) for a gas-film coefficient $(\varepsilon \sim 0.5)$, of 16.7 determined by Miyauchi et al. (80) for a liquid-film coefficient $(\varepsilon \sim 0.4)$, and of 10.0 determined by Gunn and Souza (72 ) for a gas film coefficient $(\varepsilon \sim 0.4)$.

It should be noted that Sфrensen and Stewart's results have been previously misunderstood. Their calculations do not imply a dimensionless film coefficient of 3.9 as has been claimed. Their Nusselt 
number $\mathrm{Nu}$ is based on the $\log$ mean average of the convective energy flux entering and leaving the bed. This number is not the low Péclet number, one-dimensional film coefficient.

Summary

It has been demonstrated that the low Péclet number behavior of the Sherwood number in a packed bed reactor is dependent upon its defining equation. A rigorous singular perturbation approach coupled with heuristic arguments indicates that for a deep bed the effective mass-transfer coefficient (defined by equation 1 ) is directly proportional to the Péclet number. The film coefficient (defined by equation 3) approaches a constant in the same limit. These conclusions are independent of the detailed geometric void structure in the bed. 


\section{Chapter 5}

Numerical Calculations for the Asymptotic, Diffusion Dominated Mass-Transfer Coefficient in Packed Bed Reactors

Abstract

For deep beds, the effective Sherwood number becomes linearily proportional to the Péclet number as the Péclet number tends to zero. A sinusoidal periodically constricted tube model for the voids in the bed has been used to predict the constant of proportionality. This constant depends upon the dimensionless ratios of three lengths: the average tube radius, the oscillation amplitude, and wavelength.

\section{Introduction}

In Chapter 4 a formal method for calculating the Sherwood number in the low Péclet number reglme for a mass-transfer limited, packed bed reactor was presented. Emphasis was placed on the important distinction at 1ow Péclet numbers between the effective Sherwood number, which relates the inlet and outlet concentrations, and the film Sherwood number, which is a sink term in the one dimensional model of the bed. A singular perturbation solution for the concentration field was used to demonstrate that to first order in deep beds, the effective Sherwood number can be written

$$
\frac{\varepsilon \mathrm{k}_{\mathrm{m}}}{\mathrm{a} \bar{D}_{\mathrm{o}}}=\frac{\mathrm{v}}{\mathrm{a} \bar{D}_{\mathrm{o}}}\left(\alpha_{1}-\frac{\varepsilon}{\mathrm{aL}} \ln \frac{\mathrm{v}}{\alpha_{2} \mathrm{a}_{\mathrm{o}}}\right)
$$


where $\alpha_{1}$ and $\alpha_{2}$ are constants which depend only upon the bed structure and are independent of the Pêclet number. Equation 1 applies for any detailed void volume arrangement of the bed.

In order to introduce a predictive capability to the formalism presented in that chapter, a microscopic model for the solid-void structure of the bed must be introduced. In this chapter, values of $\alpha_{1}$ are calculated using the periodically constricted tube (PCT) model of the bed.

Periodica11y Constricted Tube Mode1

The voids in a bed of nonconsolidated porous media can be modeled as an array of periodically constricted tubes. This concept has been developed by Payatakes et al. (29) and the references therein. In this work, the bed is modeled as an array of sinusoidal PCT (figure 3.2).

The first order solution for the concentration variable in the reactive section of the bed has been shown to be governed by Laplace's equation. The concentration variable within the model microscopic void volume will thus satisfy Laplace's equation.

$$
\frac{\partial^{2} \bar{\theta}}{\partial z^{2}}+\frac{\partial^{2} \bar{\theta}}{\partial r^{2}}+\frac{1}{r} \frac{\partial \bar{\theta}}{\partial r}=0
$$

The limiting reactant condition on the surface of the solid particles immediately specifies one boundary condition

$$
\bar{\theta}\left(r=r_{w}\right)=0
$$


In the we11-developed mass-transfer regions of the bed the fractional decrease of the reactant concentration and flux per period will be independent of position. This supplies the following boundary conditions

$$
\begin{gathered}
\bar{\theta}(r, 1)=\bar{\theta}(r, 0) e^{-\lambda} \\
\frac{\partial \bar{\theta}(r, 1)}{\partial z}=\frac{\partial \bar{\theta}(r, 0)}{\partial z} e^{-\lambda}
\end{gathered}
$$

where $\lambda$ is the smallest eigenvalue of the solution to equations $2,3,4$ and 5. The solution to this problem depends on the geometric parameters of the microscopic model $r_{A}$ and $A / r_{A}$.

For a deep bed, the eigenvalue $\lambda$ can be related to the leading term of equation 1 . The concentration variable at the exit of the reactor can be written as

$$
\theta_{L}=\alpha_{2} e^{-\lambda \frac{L}{l}}
$$

The PCT model yields the following geometrical relationship

$$
\frac{\mathrm{L}}{\ell}=\frac{{ }_{\mathrm{A}}}{2} \frac{\mathrm{aL}}{\varepsilon} \frac{2 \varepsilon}{\mathrm{ar}_{\mathrm{Ad}}} \text {. }
$$

Equations 6 and 7 can be substituted into the defining equation of the effective mass-transfer coefficient (eq. 4.1) to yield the analog of equation 1 .

$$
\frac{\varepsilon \mathrm{k}_{\mathrm{m}}}{\mathrm{a} D_{\mathrm{o}}}=\frac{\mathrm{v}}{\mathrm{a} D_{\mathrm{o}}}\left[\frac{\lambda \mathrm{r}_{\mathrm{A}}}{2} \frac{2 \varepsilon}{\mathrm{ar} \mathrm{Ad}_{\mathrm{Ad}}}-\frac{\varepsilon}{\mathrm{aL}} \ln \frac{\mathrm{v}}{\varepsilon \mathrm{a} \overline{\mathrm{o}}_{\mathrm{o}} \alpha_{2}}\right]
$$


Thus the leading term $\alpha_{1}$ of equation 1 can be calculated by finding the eigenvalue $\lambda$.

Method of Solution

An analytic solution for the eigenvalue problem determined by equations $2,3,4$, and 5 could not be found. An approximate numerical scheme was used. The technique is similar to that utilized in chapter 3 . Laplace's equation and the boundary conditions are transformed into a new coordinate system $(\eta, z)$ where $\eta=r / r_{w}(z)$. The wall boundary condition is then shifted to the coordinate curve $\eta=1$. A transformed concentration variable $\mathcal{C}$ was defined as

$$
C=\bar{\theta} e^{\lambda z}
$$

This transformation was introduced in order to remove the eigenvalue from the boundary conditions and transfer it to the differential equation. Laplace's equation and its boundary conditions are then written as

$$
\begin{gathered}
\mathcal{C}(\eta, 0)=\mathcal{C}(\eta, 1) \\
\frac{\partial \mathcal{C}(\eta, 0)}{\partial z}=\frac{\partial \mathcal{C}(\eta, 1)}{\partial z} \\
\mathcal{C}(1, z)=0 \\
\frac{\partial^{2} C}{\partial z^{2}}+\left[\frac{1}{r_{w}^{2}}+\eta^{2}\left(\frac{r_{w}^{\prime}}{r_{w}}\right)^{2}\right] \frac{\partial^{2} c}{\partial \eta^{2}}+\left[\frac{1}{\eta r_{w}^{2}}+\eta\left(2\left(\frac{r_{w}^{\prime}}{r_{w}}\right)^{2}-\frac{r_{w}^{\prime \prime}}{r_{w}}+\right.\right. \\
\left.\left.2 \frac{r_{w}^{\prime}}{r_{w}} \lambda\right)\right] \frac{\partial C}{\partial \eta}-\left[2 \eta \frac{r_{w}^{\prime}}{r_{w}}\right] \frac{\partial^{2} C}{\partial \eta \partial z}-2 \lambda \frac{\partial C}{\partial z}+\lambda^{2} c=0 .
\end{gathered}
$$


Two independent collocation procedures were used to solve equations 10 thru 13. These two techniques permitted a cross verification of the calculated results.

In the first method, an expansion for $C$ was assumed in the form

$$
C(n, z)=\sum_{k=1}^{N C P} A_{k}(z) J_{o}\left(\gamma_{k} \eta\right)
$$

Since the $\gamma_{k}$ are the roots of the Bessel function $J_{0}$, this expansion identically satisfies the wall boundary condition. Equation 14 is substituted into equation 13 , and the residual is made equal to zero at NCP $\eta$ collocation points. This generates a system of ordinary differential equations with the following boundary conditions

$$
\begin{aligned}
& A_{k}(0)=A_{k}(1) \\
& A_{k}^{\prime}(0)=A_{k}^{\prime}(1) .
\end{aligned}
$$

Along with the normalization

$$
A_{1}(0)=1
$$

this specifies enough information to calculate the unknowns. Equation 13 was linearized and then solved by iteration on a $z$ finite-difference grid using the method of Newman (63) slightly modified to exploit the storage space savings made possible by the periodic boundary conditions (Appendix C).

The second method uses a double series expansion to transform the original partial differential equation into a system of algebraic equations. 
Since the $A_{k}$ functions are periodic, they can be expanded in a Fourier series

$$
A_{k}(z)=\sum_{m=1}^{N F C}\left(a_{k m} \cos 2 \pi(m-1) z+b_{k m} \sin 2 \pi m z\right) .
$$

The periodicity conditions are then identically satisfied. The unknown Fourier coefficients are determined by collocating the residual of a linearized equation 13 on a grid of NCP $\eta$ points and 2NFC $z$ points. These collocation equations and the normalization condition specify a determinate system.

In both techniques the $\eta$ collocation points were chosen to be evenly spaced in $\eta^{2}$ in the open interval $(0,1)$. The $z$ points used in the double collocation method were chosen to be evenly spaced in the semi-open interval $[0,1)$. The collocation/finite difference method was computationally advantageous for the larger values of the parameters reported here.

The computer programs are given in Appendix $B$. Results and Discussion

As seen from equation 8, the important quantity for calculating the Sherwood number is $\lambda \mathrm{r}_{\mathrm{A}}$. Figure 5.1 presents the calculated values of $\lambda \mathrm{r}_{\mathrm{A}}$ in a sinusoidal PCT normalized by the value of $\lambda \mathrm{r}_{\mathrm{A}}(=2.40482)$ for a straight wall cylinder.

Figure 5.2 presents results for the leading term of the Stanton number $\left(\mathrm{Sh}_{\mathrm{B}} / \mathrm{Pe}_{\mathrm{B}}\right)$ in a mass-transfer controlled, deep-bed packed reactor modeled as an array of sinusoidal PCT. As the sinusoidal tube 


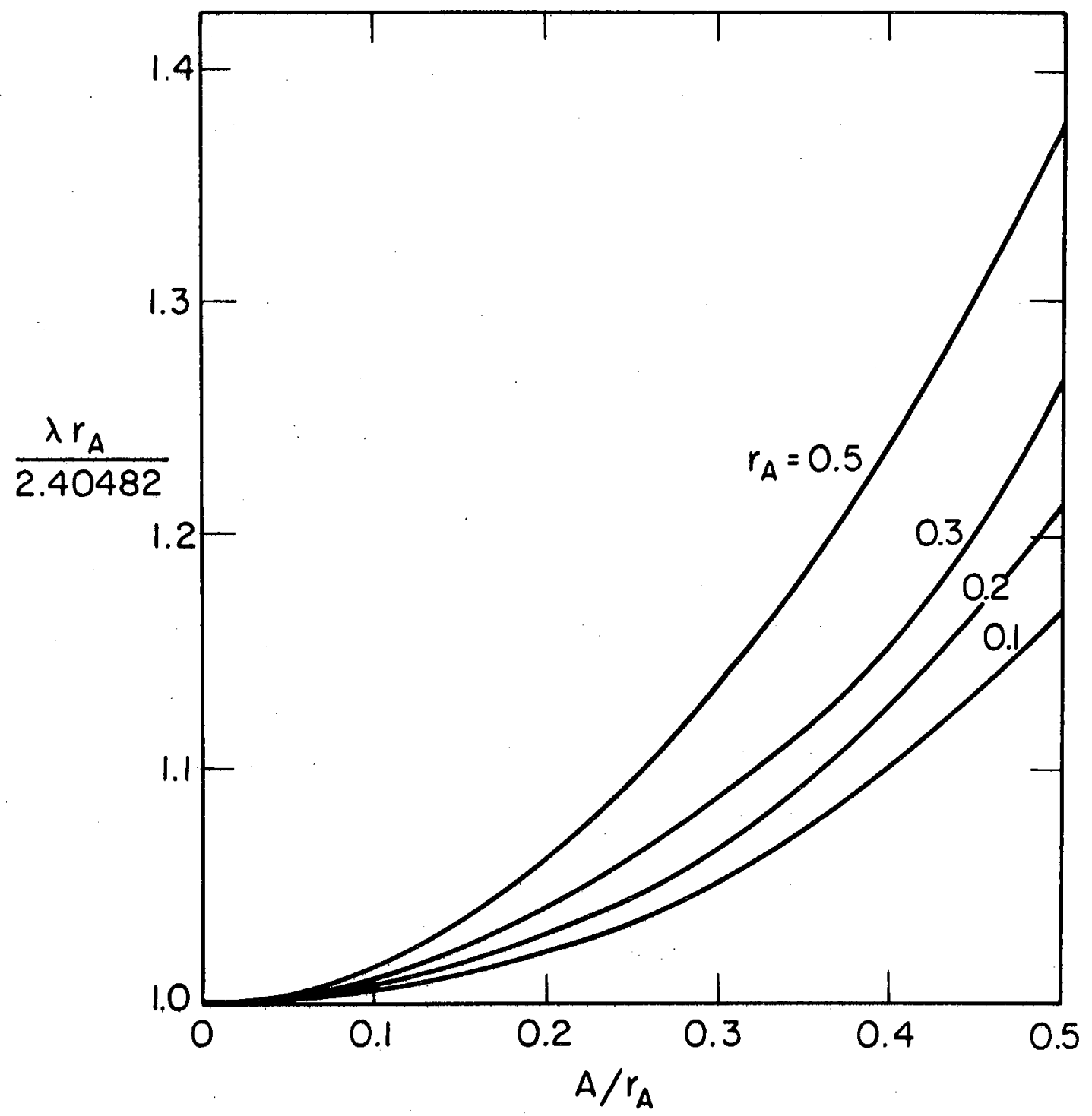

XBL $7711-6485$

Figure 5.1 The first eigenvalue of Laplace's equation in a sinusoidal PCT. 


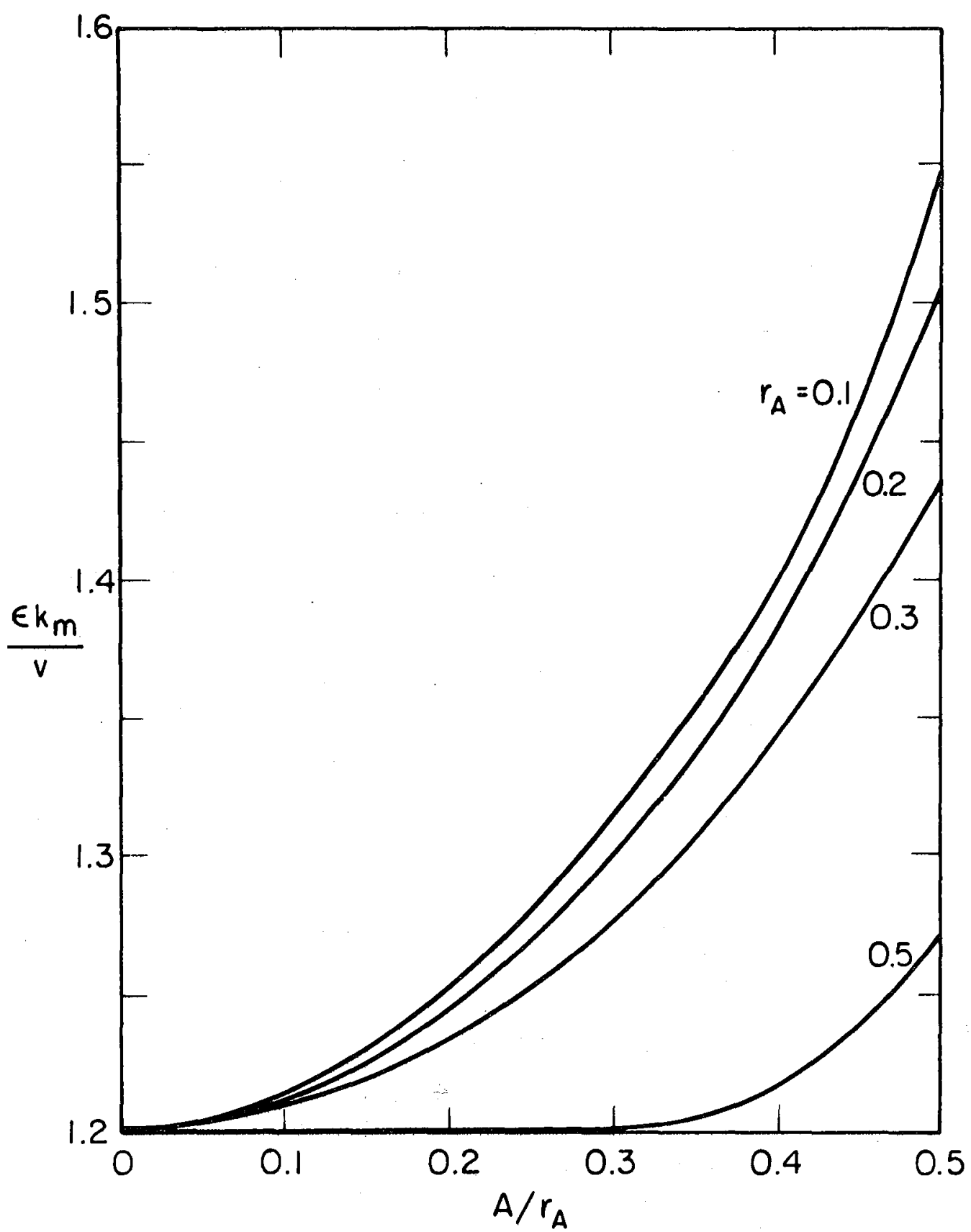

XBL $7711-6486$

Figure 5.2 Calculated values of the Stanton number for a mass-transfer controlled packed bed reactor in the low Péclet number region. 
amplitude approaches zero, for all values of $r_{A}$ the Stanton number approaches 1.202 , the value for a straight wall tube.

S $\phi$ rensen and Stewart (12) have calculated the asymptotic Stanton number for a bed of uniform sized spheres in simple cubic packing. In the terminology of this work, that number is found to be 1.233. As was found in the high Péclet number, asymptotic Sherwood number calculations and the friction factor calculations, a value of $r_{A} \sim 0.5$ and $\mathrm{A} / \mathrm{r}_{\mathrm{A}} \sim 0.3$ to 0.4 for a sinusoidal $\mathrm{PCT}$ reproduce satisfactorily Sфrensen and Stewart's results.

As has been emphasized, the above solution only generates the leading term of the deep-bed Stanton number. It is necessary to ask under what conditions can the second term of equation 1 be neglected. In order to calculate $\alpha_{2}$, the concentration variable in the entrance region of the bed must be calculated. This is an order of magnitude more difficult problem and is not attempted here. S $\phi$ rensen and Stewart, however, have solved this problem for simple cubic packing of uniform spheres. We can use their results to estimate the effect of neglecting the second term in the expansion. Table 5.1 shows that for most bed depths with a Péclet number greater than 0.001 , the error is acceptable. The error is seemingly further diminished in scale when a $\log -10 g$ plot of $\mathrm{Sh}_{\mathrm{B}}$ vs $\mathrm{Pe}_{\mathrm{B}}$ is examined.

The Stanton numbers presented above are for a non-diluted reactive bed. The reactive section of the bed must not contain an excess of inert particles. It should be emphasized that only under these conditions does Laplace's equation describe the concentration variable 
Table 5.1

Sørensen and Stewart's results for the deep-bed, low Péclet number Stanton number of a simple cubic packed lattice of uniform size spheres

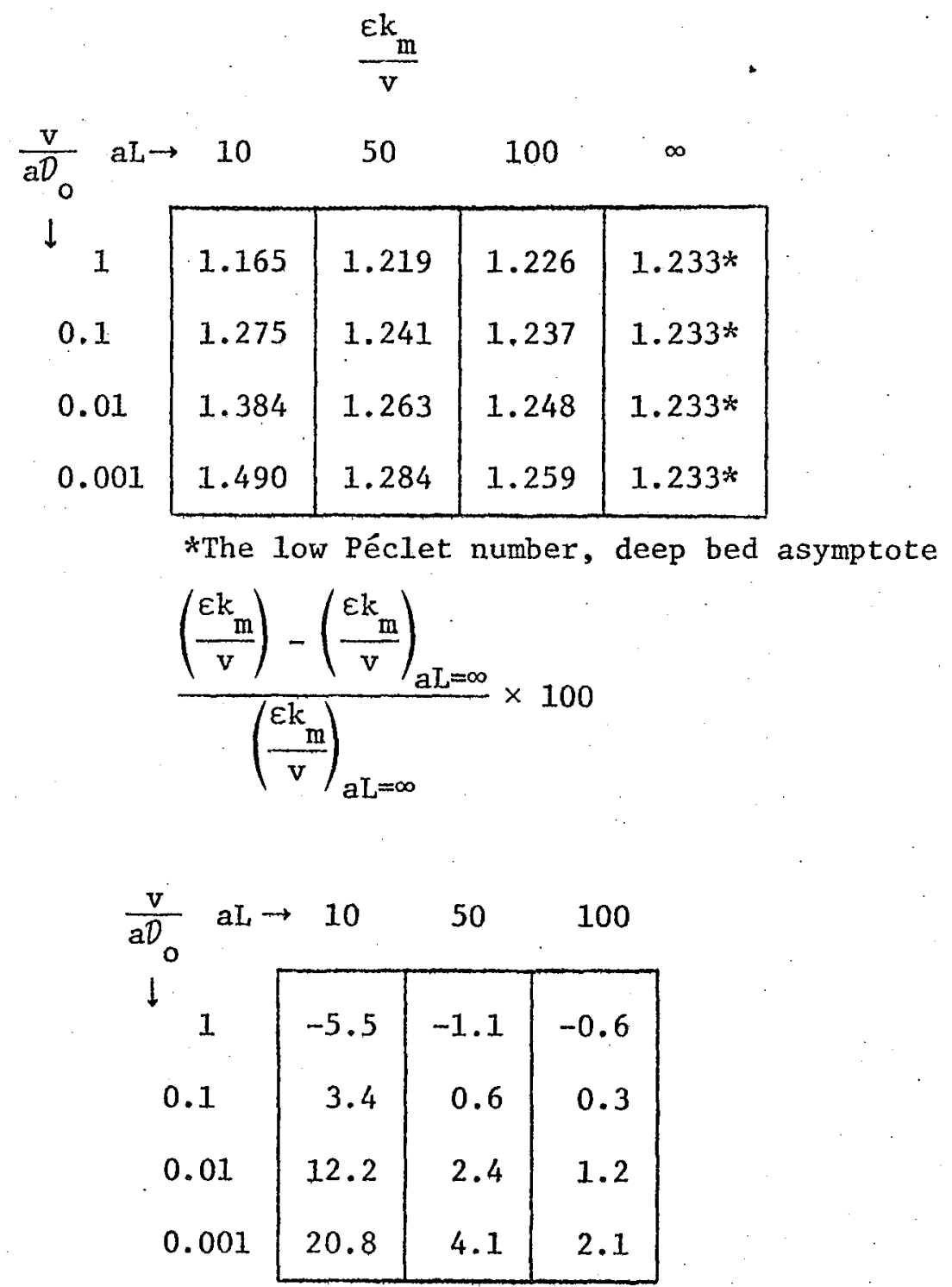


in the reactive section of the bed. For two beds with identical $\varepsilon$ and aL values and with the same feed flowrate and concentration, one may argue, qualitatively, that the effective mass-transfer coefficient in the non-diluted bed must be greater or less than that in the diluted bed. Care must be taken in extrapolating low Péclet number mass-transfer coefficient experiments in diluted beds to non-diluted beds. 


\section{Chapter 6 \\ Entrance Region (Lévêque-1ike) Mass Transfer Coefficients in Packed Bed Reactors}

\section{Abstract}

Calculations for the high Péclet number, entrance region (Lévêque-1ike) packed bed, mass-transfer coefficient using a sinusoidal periodically constricted tube model for the void structure of the bed are presented. An inverse cube root dependence of the mass-transfer coefficient on the bed depth is predicted. This length dependence is anticipated only at very low Reynolds numbers. Calculations which assume a mixing region between successive periods are also presented. No bed length dependence is anticipated in these coefficients.

\section{Introduction}

The periodically constricted tube is a useful model of void structure, in calculation of mass transfer in packed bed reactors. This model was developed by Payatakes and co-workers (Payatakes et a1., 29, 30,31 ) to predict the permeability of a nonconsolidated packed bed. They envisioned the bed as cell structures made of segments of parabolic periodically constricted tubes. A sinusoidal periodically constricted tube (PCT) is used in this work to model the void structure in a bed in order to predict the mass-transfer coefficient. The fluid is assumed to be in the viscous flow regime, and the reactant conversion is assumed to be controlled by mass transfer from the fluid to the particle surface. 
In earlier chapters calculations were presented using this model for the mass-transfer coefficient in the high and low Péclet number, deep-bed asymptotic limit. These limiting values can be used in their appropriately defined ranges (as suggested by Karabelas et a1., 71 ), or they can be empirically combined to cover the intermediate Péclet number range. This approach is similar to that taken by Sorensen and Stewart (11,12) who in their pioneering work solved the convectivediffusion equation in a simple cubic packed bed of uniform sized spheres. In this chapter, the high Péclet number, entrance region (Lévêque-1ike) mass-transfer coefficient calculations are presented. The term "entrance region" is used here to designate that region where a concentration boundary layer has started to grow along the wall of the packing or particles but has not yet completely filled the flow passage.

A note similar to this was presented by Tardos et al. (21). They presented calculations for the mass-transfer coefficient under the stated restrictions using various sphere-in-a-cell models for the void structure in the bed. As will be seen, in the creeping flow regime these models are inherently different from the conduit model, which predicts a length dependent coefficient.

\section{Mathematical Formulation}

Figure 3.2 represents a segment of a sinusoidal PCT. The bed is imagined to be a matrix of these tubes. The well-known straight conduit model results when the amplitude of the tube wall oscillation 
equals zero. The tube parameters may be determined by the procedure suggested by Payatakes et $\underline{\text { al }}$. (29).

The effective mass-transfer coefficient $k_{m}$ is defined as follows

$$
\frac{c_{L}}{c_{F}}=\exp \left(-a k_{m} L / v\right)
$$

where $c_{F}$ and $c_{L}$ are the reactant concentration far upstream and downstream of the reactor, respectively. Since the axial dispersive flux becomes negligible compared to the convective flux at high Péclet numbers, equation 1 can be generated by integrating the one-dimensional model for the bed wherein $k_{m}$ is analogous to a first-order rate constant. The distinction between this coefficient and the film coefficient $k_{f}$ which appears as the first-order rate constant in the one-dimenstonal model of the bed which includes a dispersive flux should be pointed out. Only in the high Péclet number limit do these two coefficients become indistinguishable.

Consider a single PCT of length L . A mass balance for the reactant across the tube (inlet-to-outlet) can be written as

$$
\mathrm{q}_{\mathrm{T}}\left[\mathrm{c}_{F}-\mathrm{c}_{\mathrm{L}}\right]=\begin{aligned}
& \text { rate of moles reacted } \\
& \text { at tube wall }
\end{aligned}
$$

where $q_{T}$ is the flowrate per tube. The bed is assumed to be homogeneous, thus the reactant concentrations of equation 2 are equal to those of equation 1 . The right side of equation 2 can be calculated by solving the appropriate form of the convective-diffusion equation. We shall demonstrate shortly how this calculation is carried out, but 
let us express the result of this step in terms of an average Sherwood number for the tube,

$$
<\mathrm{Sh}>=\left\langle\mathrm{N}_{\mathrm{w}}>\frac{2 \mathrm{r} A \mathrm{Ad}}{D_{\mathrm{o}} \mathrm{c}_{F}}\right.
$$

where $\left\langle\mathrm{N}_{\mathrm{w}}\right\rangle$ is the average flux over the surface area contained in length $\mathrm{L}$. In the high Péclet number limit, combining equations 1 , 2 , and 3 yields

$$
\frac{\mathrm{k}_{\mathrm{m}} \mathrm{aL}}{\mathrm{v}} \cong \frac{<\mathrm{Sh}>}{2 \mathrm{r}_{\mathrm{Ad}}<\mathrm{v}_{\mathrm{Ad}}>/ 0_{\mathrm{o}}} \frac{\mathrm{SA}_{\mathrm{L}}}{\pi \mathrm{r}_{\mathrm{Ad}}^{2}}
$$

where $\mathrm{SA}_{\mathrm{L}}$ is the surface area contained in length $\mathrm{L}$. The bed Sherwood number (a dimensionless mass-transfer coefficient) can be written by relating the superficial velocity $v$ to the average velocity in the tube $\left\langle\mathrm{v}_{\mathrm{Ad}}\right\rangle$

$$
{ }_{\mathrm{Ad}}>=\frac{\mathrm{v}}{\varepsilon}\left[1+\frac{1}{2}\left(\mathrm{~A} / \mathrm{r}_{\mathrm{A}}\right)^{2}\right]
$$

We then find

$$
\mathrm{Sh}_{\mathrm{B}}=\frac{\varepsilon \mathrm{k}_{\mathrm{m}}}{\mathrm{a} D_{\mathrm{O}}}=\frac{\langle\mathrm{Sh}>}{4}\left(\frac{2 \varepsilon}{\mathrm{ar}_{\mathrm{Ad}}}\right)^{2}\left\{\frac{\mathrm{SA}_{\mathrm{L}}}{2 \pi \mathrm{r}_{\mathrm{Ad}} \mathrm{L}\left[1+\frac{1}{2}\left(\mathrm{~A} / \mathrm{r}_{\mathrm{A}}\right)^{2}\right]}\right\}
$$

In order to calculate the mass-transfer coefficient from equation 6 , a value for the average sherwood number $(<\mathrm{Sh}>)$ in a tube must be determined. This may be found by applying a Lighthill transformation (84) to the convective-diffusion equation. The 
axial diffusive flux is assumed to be negligible, and the velocity profile is taken to be linear near the wall. The governing equation may be found in Newman's text (85) and is reproduced below.

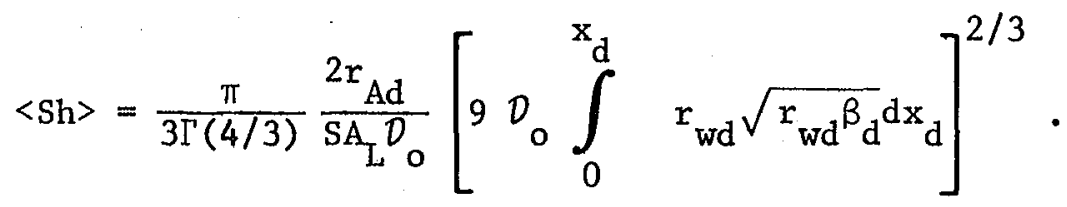

The subscript $d$ indicates a dimensional quantity. The integral is carried out along the boundary-layer coordinate $x$, measured along the surface of the tube. The radius of the tube is $r_{w d}$, and $\beta_{d}$ is the normal derivative of the velocity at the wall. This may be found by appropriate differentiation of the stream function solutions found in Chapter 3. In a dimensionless form this derivative is expressed as

$$
r_{A} \frac{\partial v_{t}}{\partial n}=4\left(\frac{{ }^{r} A}{r_{w}}\right)^{3}\left(1+r_{w}^{\prime} 2\right)\left[1-\sum_{k=1}^{N C P} A_{k}(z) \phi_{k}(1)\right] \text {. }
$$

Equation 7 may be inserted into equation 6 and, after some rearranging, this results in an expression for the macroscopic quantity $\mathrm{k}_{\mathrm{m}}$ in terms of the measurable parameters $\mathrm{aL}, \varepsilon, \mathrm{Pe}_{\mathrm{B}}$, and the microscopic model parameters $\mathrm{r}_{\mathrm{A}}$ and $\mathrm{A} / \mathrm{r}_{\mathrm{A}}$

$$
\frac{\varepsilon k_{m}}{a D_{o}}=\frac{9^{2 / 3}}{3 \Gamma(4 / 3) 4^{1 / 3}} I^{2 / 3}\left[\frac{2 \varepsilon}{\operatorname{ar} \operatorname{Ad} \sqrt{1+\frac{1}{2}\left(A / r_{A}\right)^{2}}}\right]^{4 / 3}\left(\frac{\varepsilon v}{\mathrm{aLa}_{\mathrm{o}}}\right)^{1 / 3}
$$

where 


$$
I=\int_{0}^{\frac{1}{2}} \frac{r_{w}}{r_{A}} \sqrt{1+r_{w}^{\prime 2} \frac{r_{w}}{r_{A}} r_{A} \frac{\partial v_{t}}{\partial n}} d z .
$$

It has been assumed in deriving equation 9 that $L / \ell$ is an integer.

\section{Results and Discussion}

The bed Sherwood number may be calculated by evaluating the integra1 $I$ of equation 10 by the use of equation 8 . In the course of the calculations, it was found that $\partial v_{t} / \partial n$ became less than zero for certain ranges of the tube parameters. This implies separation flow. Separation in viscous flow has been reported in the 1iterature by Davis and $0^{\prime}$ Neil (86), Moffat (87), and Ganatos et al. (88), among others. The Lighthill transformation is not valid when the shear rate becomes negative.

In the worse case for which calculations are presented $\left(r_{A}=1 / 2, A / r_{A}=1 / 2\right)$, the surface area of the tube occluded by the separation zone is 44 percent of the total surface area. Figure 6.1 presents the streamlines for this case. In the spirit of numerical simplicity, the shear rate was set identically equal to zero from the separation point to $z=1 / 2$ in evaluating the integral $I$, thus neglecting the complications caused by the flow pattern. This will result in an underestimate in $k_{m}$. Those values of the tube parameters for which separation was found are indicated by the dashed line of figure 6.2 and 6.3 .

Figure 6.2 is a plot of the integral I . Figure 6.3 is a plot of the high-Péclet-number, entrance-region Sherwood number, Both figures use $r_{A}$ and $A / r_{A}$ as parameters. 


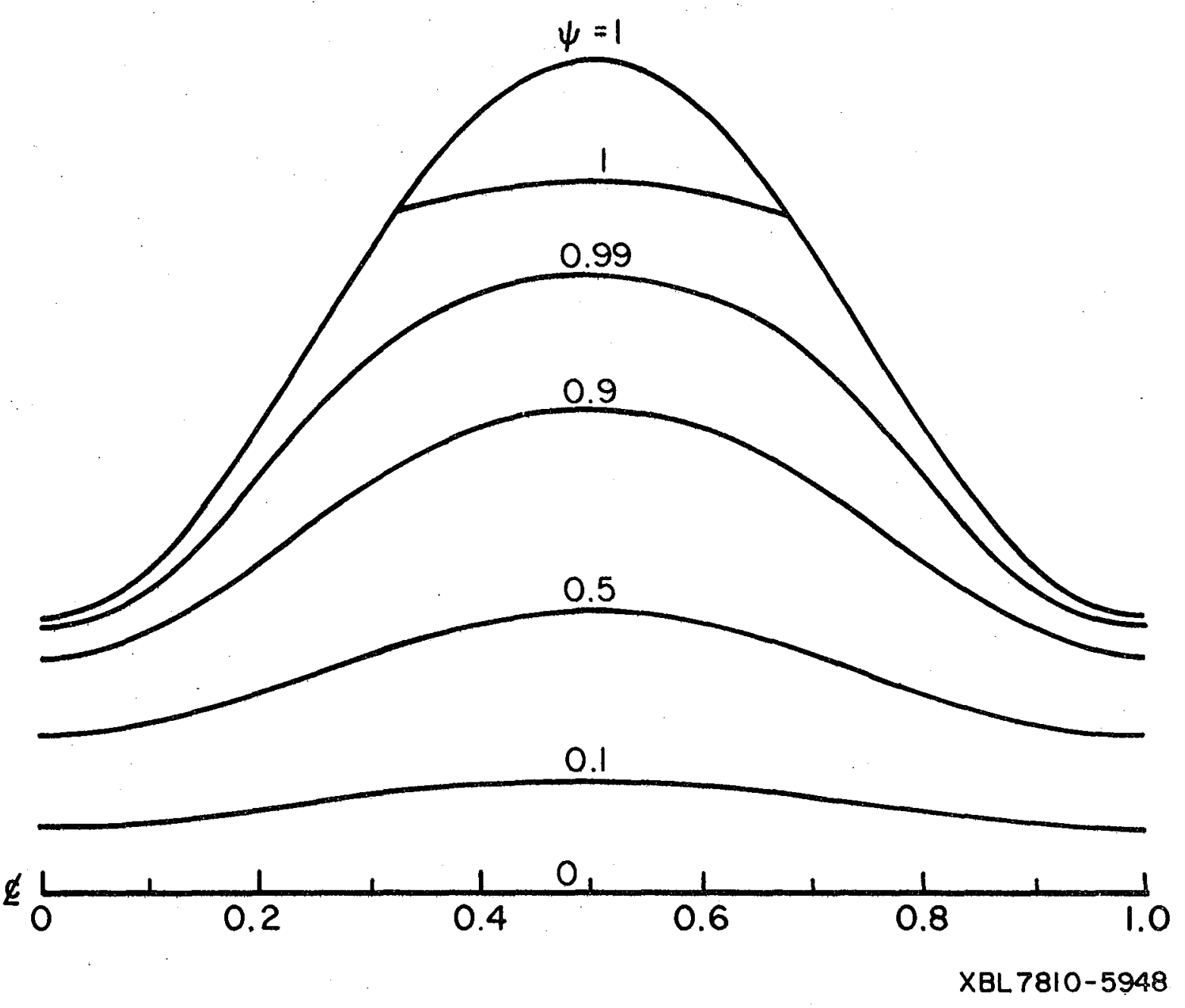

Figure 6.1 Streamlines in a sinusoida1 PCT with $\mathrm{r}_{\mathrm{A}}=0.5, \mathrm{~A} / \mathrm{r}_{\mathrm{A}}=0.5$. 


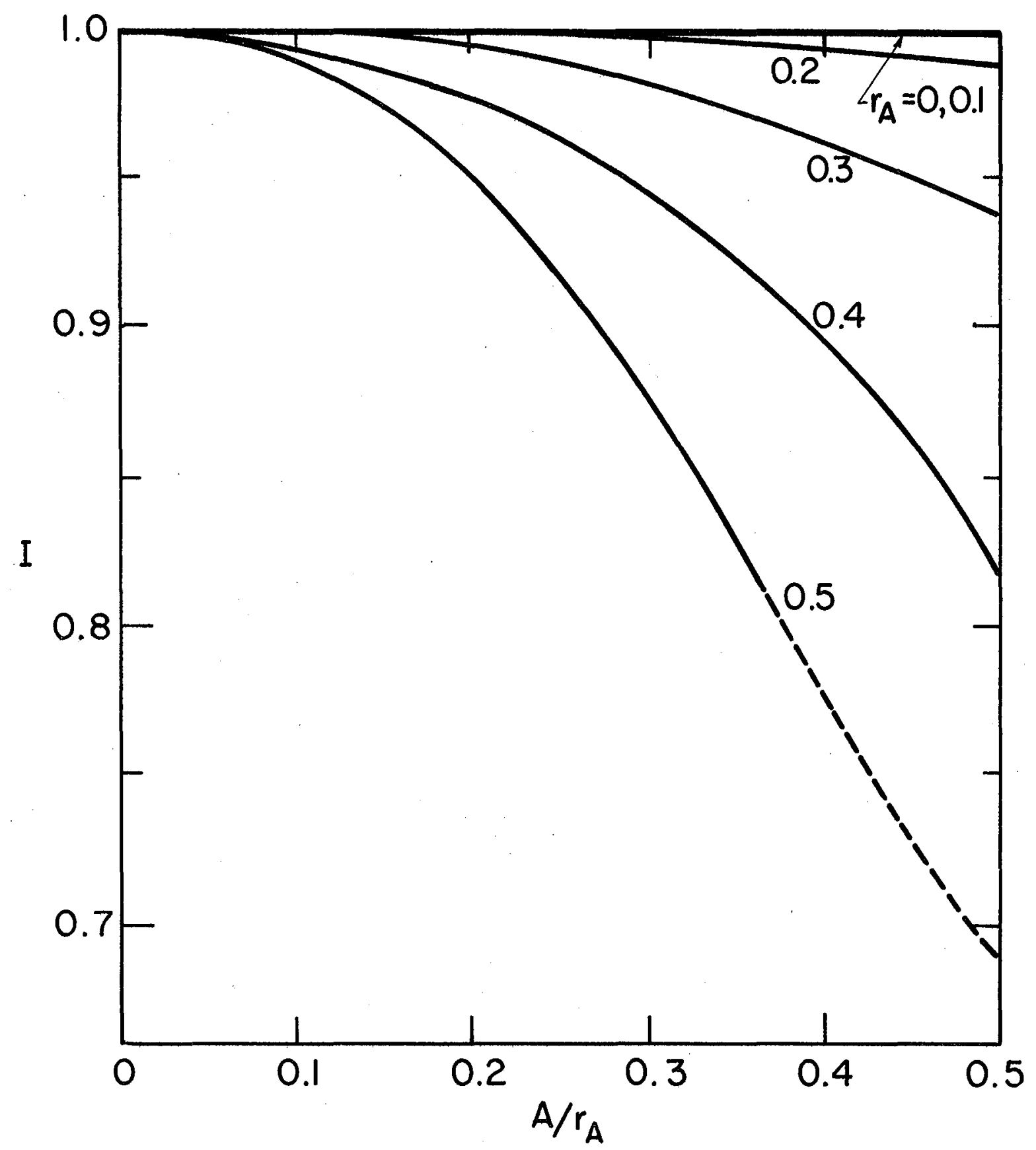

XBL7810-5947

Figure 6.2 The integral I plotted as a function of the sinusoidal PCT parameters. 


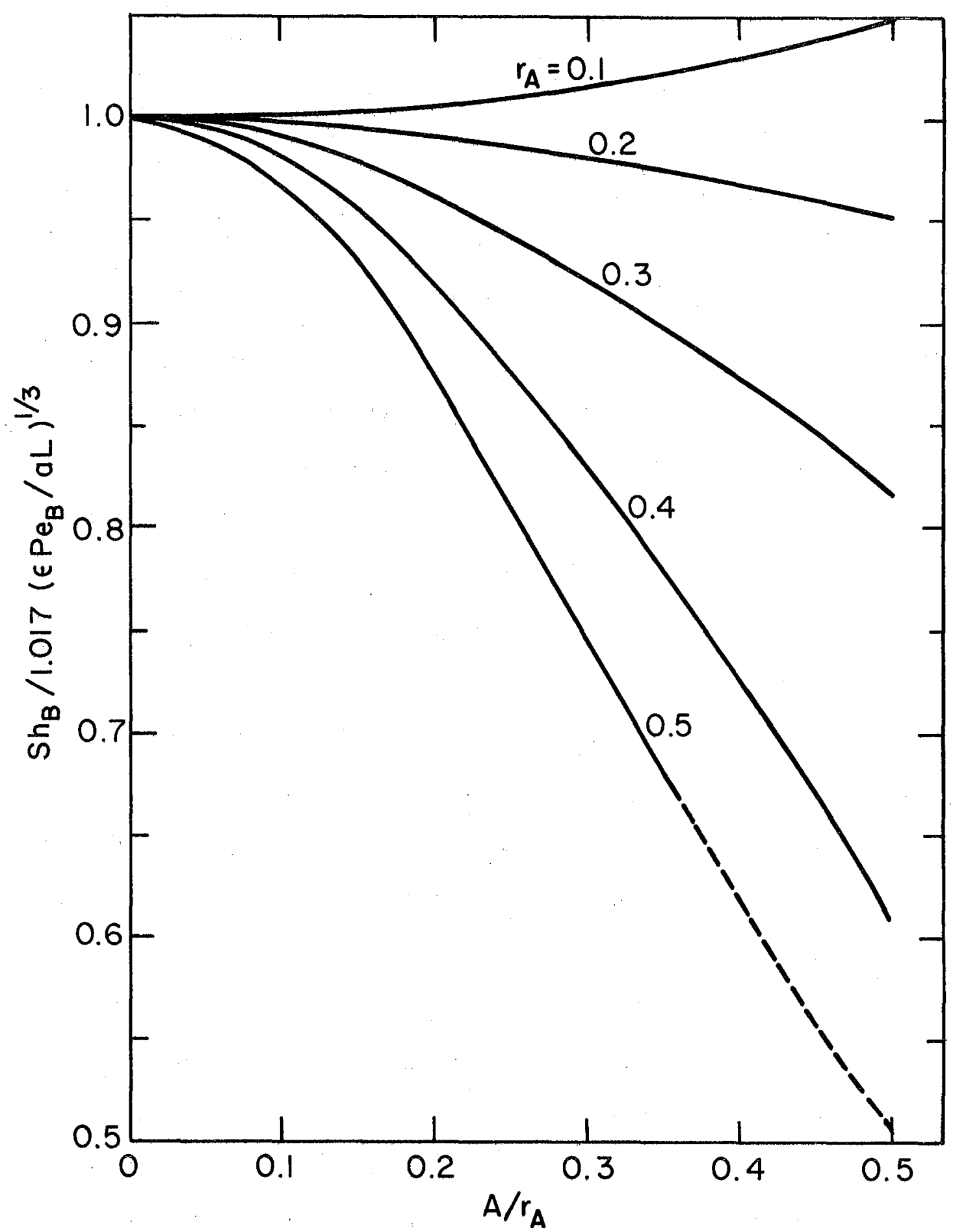

XBL7810-5946

Figure 6.3 Packed bed, entrance region mass transfer coefficient as a function of the sinusoidal PCT parameters. 
As was found by Chow and Soda (89), who solved a regular perturbation problem for small values of $\mathrm{r}_{\mathrm{A}}$, the Sherwood number increases with amplitude. However, for larger values of $r_{A}$ this trend reverses itself. This identical trend was found for the high Péclet number, deep-bed asymptote.

Equation 8 predicts that the bed Sherwood number decreases as $L^{-1 / 3}$ for a mass-transfer controlled reaction. S $\phi$ rensen and Stewart (12) have also reached the same conclusion. One cannot find conclusive evidence in the literature to substantiate this prediction. Kato et al. (49) have correlated their data with a packing depth factor. There are also indications of a length dependent $k_{m}$ in the data of Wilson and Geankopolis (52) and of Alkire and Gracon (37). For a given flowrate, increasing the packing depth by a factor of 10 will result in a decrease of $k_{m}$ by 54 percent. Considering the nature of experimental measurements of $k_{m}$, this diffference can easily be obscured by experimental error. Thus an experiment to ascertain if there is a packing depth effect must be carefully designed.

Tardos et al. (21) compared their calculations for $k_{m}$ with the experimental correlation of Wilson and Geankopolis. This latter correlation was developed from data taken from beds with aL values ranging from 3.4 to 27 with $\varepsilon$ approximately 0.4 . If an average aL of 15 is assumed, the PCT model predicts the ratio $\mathrm{Sh}_{B} / \mathrm{Pe}_{B}^{1 / 3}$ to be in the range 0.214 to 0.304 while Tardos et al. report a range of this ratio from 0.536 to 0.584 depending upon the cell model used. The Wilson and Geankopolis correlation results in a value of 0.464 . 
It should be emphasized that the packing-depth effect is anticipated only at low Reynolds numbers. In creeping flow, the Péclet number is the only physico-chemical parameter controlling the masstransfer rate. As the flowrate increases, the Reynolds number also becomes a factor to consider. In the non-viscous flow region, mixing eddies will become a dominant flow structure in the intersticies of the packing. Thus any boundary layer that might form on the surface of the particles is destroyed by the eddies. In this case, the models of a sphere in a cell become physically more appropriate. The conduit model may also be applied here by redefining the length scale over which the average tube Sherwood number is calculated. Kataoka et al. (25) have carried out such an analysis using the straight tube model. With the aid of figure 6.2 , it is possible to carry out such an analysis for the sinusoidal PCT.

The fluid is now imagined to be well mixed before it enters a period and remixed after it leaves a period. The Lévêque solution can be applied in each period to calculate the mass-transfer coefficient. The length scale over which the Lighthill transformation is applied is $\ell$ In this case rather than $L$. Equation 9 still applies after the appropriate substitution. The period length $\ell$ may be related to the particle diameter by

$$
\ell=\left[\frac{\pi}{6(1-\varepsilon)}\right]^{1 / 3} \mathrm{~d}_{p}
$$

as suggested by Payatakes et a1. Substituting into equation 10 and introducing the specific interfacial area for $d_{p}$, one obtains 


$$
\frac{\varepsilon \mathrm{k}_{\mathrm{m}}}{\mathrm{a} \bar{D}_{\mathrm{o}}}=\frac{9^{2 / 3}}{3 \Gamma(4 / 3) 4^{1 / 3}}\left\{\frac{\varepsilon}{[6(1-\varepsilon)]^{2 / 3}}\right\}^{1 / 3}\left[\frac{2 \varepsilon}{\operatorname{ar} \mathrm{Ad} \sqrt{1+\frac{1}{2}\left(\mathrm{~A} / \mathrm{r}_{\mathrm{A}}\right)^{2}}}\right]^{4 / 3} I^{2 / 3}\left(\frac{\mathrm{v}}{\mathrm{a}_{\mathrm{o}}}\right)^{1 / 3}
$$




\section{Chapter 7}

The Measurement of Mass-Transfer Controlled Reaction Rates in an Electrochemical Packed Bed Reactor

\section{Purpose}

In this chapter an experimental program to measure transport controlled mass-transfer coefficients is discussed.

\section{Summary of Procedure}

A randomly packed bed of uniform size, copper plated, stainless steel bearings was used as the cathode in an electrochemical, flowthrough reactor. Copper was plated on the surface of these particles from an acidified ( $1 \mathrm{M} \mathrm{H}_{2} \mathrm{SO}_{4}$ ) copper sulfate solution. Copper deposition was chosen as the test reaction because atomic adsorption can be used to measure accurately the ion concentration at $0.1 \mathrm{ppm}$ with an uncertainty of \pm 1 percent. The evolution of $\mathrm{O}_{2}$ in a separate compartment was the anode reaction. A sufficient cathodic polarization was applied to the bed to ensure that the deposition reaction was controlled by the transport of the $\mathrm{Cu}^{++}$ions to the particle surface. This transport controlled reaction manifests itself as a limiting current plateau on a current versus applied potential plot. The overall reaction rate for the copper deposition can be measured by two independent techniques: $i$ ) the inlet and outlet $\mathrm{Cu}^{++}$concentration is determined and ii) the cell current is measured. The latter is, according to Faraday's Law (assuming negligible side reactions), proportional to the amount of copper consumed. These two independent measurements permit a cross verification of the mass-transfer coefficients calculated from the data. Only those data which give mass-transfer coefficients which deviate 
\pm 5 percent from the average are accepted. All other data were rejected. The experimental variables which were manipulated were the flowrate of the feed, the Schmidt number of the feed (by addition of glycerol), and the packing depth. The Reynolds ( $/$ av) number range varied from 0.198 to 0.00271 ; two values of Schmidt number were studied, 2000 and 9000 ; and two values of packing depth were studied, characterized by aL $=30$ and $\quad$ aL $=100$.

\section{Introduction}

As was seen in Chapter 2, the limiting current analysis of Bennion and Newman ( 40) indicates that the porous electrode design constraints of a maximum allowable ohmic drop $\left(\Delta \Phi_{2}\right)$ along with the required conversion set an upper limit on the flowrate through the bed. This flowrate for typical values of $\Delta \Phi_{2}$ corresponds to Péclet $\left(\mathrm{v} / \mathrm{aD}_{0}\right)$ numbers in the range 10 to 500 . For typical values of the Schmidt number (1000), this Péclet number range corresponds to Reynolds numbers in the range 0.01 to 0.5 .

The availability of mass-transfer data in this low flowrate region is very sparse. A review of the low Pêclet number data has already been presented in Chapters 2 and 4 . Most of these data were taken for gaseous systems.

There exists a need for reliable mass-transfer data in the low Péclet number (1ow Reynolds, high Schmidt number) region. The purpose of this experimental program is to provide data in this range. 
There are a few publications which have studied this problem. Williamson et a1. (51) and Wilson and Geankopolis (52) have studied the low Reynolds number mass-transfer behavior in a bed packed with benzoic acid spheres. Most of their data are above the 0.5 Reynolds number, and those data below this show considerable scatter. Alkire and co-workers [Alkire and Gracon (37), A1kire and $\mathrm{Ng}$ (90), Alkire and Gould (91)] have studied porous flow-through electrodes at very low Reynolds numbers. In the course of their work, they have generated limiting current curves which can be used to calculate mass-transfer coefficients. Unfortunately, they used screen material as the active packing in their beds. The interfacial area available for mass-transfer is not well defined in this geometry. Alkire et a1. have used an area adjusting factor to bring their experimental results in line with their calculations. However, even if the screen surface area were known with confidence, their results should not be used to calculate mass-transfer coefficients. This is because they measured and reported only limiting currents, and, as will be seen shortly, in the low Reynolds number region where the reactant is consumed with nearly $100 \%$ efficiency, a small uncertainty in the limiting current will cause a large error in the mass-transfer coefficient. Coeuret (92) has studied the low Reynolds number behavior in a porous flow-through electrode. He used the reduction of ferricyanide on a bed of spherical, gold plated, graphite particles. He varied the Schmidt number and packing depth. The majority of his data are above the Reynolds number region of interest. He has reported the ferricyanide conversion as well as 
the limiting current. Appel's dissertation (16) was concerned with the measurement of mass-transfer coefficients in low Reynolds number flow. In a shallow $(a L=10)$, hexagonally packed bed he studied the reduction of ferricyanide on precision stainless steel bearings. The mass-transfer coefficlent was calculated by three independent measurements. The combination of reproducible packing and the ability to cross verify the three calculated mass-transfer coefficients make his results highly reliable.

\section{The Limiting Current Technique}

The electrochemical reaction of a test species from a fluid at an electrode surface is an excellent probe for the mass transfer behavior at an interface. If a sufficient polarization is applied to the electrode, the rate of reaction is controlled by the transfer of the reactant from the bulk to the interface. This mass-transfer controlled reaction is immediately recognized by a limiting current plateau on a current versus electrode polarization curve. An excellent review of the limiting current technique is given by Selman (93).

The 1imiting current technique has been applied by many workers to the study of mass-transfer in packed beds. Besides the work of Alkire and co-workers, Appe1, and Coeuret mentioned above, others have used this tool to study packed bed mass-transfer behavior. Jolls and Hanratty (94) placed a single active sphere in a bed of inert spheres and measured the current to segmented electrodes flush on the surface. This technique gives an indication of the spatial behavior of the 
reaction rate. Karabelas et a1. (71) used a single sphere whose entire surface was active in a bed of inert particles. Mandelbaum and Böhm (95) placed six active Raching rings in a bed of inert rings and reported the average mass-transfer coefficient of all six particles. Appel has pointed out that the results of these three works should not be applied to a bed filled with active particles.

The implementation of the limiting current technique to measure mass-transfer coefficients in packed beds is decidedly more complicated than the dissolution of benzoic actd or napthalene spheres which has been used by other workers in the field, However, the added complexity of this technique does have advantages. Consider the reduction of ferricyanide in a bed of spherical particles. One can measure the inlet and outlet ferro and ferricyanide species concentrations and hence calculate two values of the mass-transfer coefficient. One can also measure the total current flowing to the cell and from this calculate another value for $k_{m}$. These three values can be used to cross verify one another, and therefore any bad data can be rejected.

Most of the experimental work reported above has used the reduction of ferricyanide as the test reaction. This reaction is popular because it does not change the surface area of the particle in the course of the reaction and it has a large exchange current density. The analytical technique (titration) used to measure the ferro/ferricyanide species is accurate to only approximately 1 part in 10 for a $10^{-4}$ molar solution. Thus, if one wants to measure accurately the inlet and outlet concentrations, the conversion in the bed must remain within certain bounds. 
This problem has been discussed by Appel (16) and by Yip (96). This bound limits the range of variables that can be varied in an experiment. For a given packing depth it sets the lowest permissible flowrate, and for a given flowrate, it determines the deepest permissible packing depth. One could forgo the concentration measurements and record only the cell current and relate this to the mass-transfer coefficient, but this will result in a loss of accuracy. This is demonstrated below.

The effective mass-transfer coefficient in the transport controlled reactor is defined as

$$
k_{m}=-\frac{v}{a L} \ln \frac{c_{L}}{c_{F}} .
$$

This can also be written with the use of Faraday's law in terms of the measured cell current $\mathrm{I}_{\mathrm{c}}$

$$
\mathrm{k}_{\mathrm{m}}=-\frac{\mathrm{v}}{\mathrm{aL}} \ln \left[1-\frac{\mathrm{I}_{\mathrm{c}}}{\mathrm{nFQc_{F }}}\right] \text {. }
$$

Any experimental measurement has uncertainty associated with it. Let the error in the measured concentrations be designated by $\varepsilon_{1}$, and that in the current by $\varepsilon_{2}$, i.e.

$$
\begin{aligned}
& c=c^{A V}\left(1 \pm \varepsilon_{1}\right) \\
& I=I^{A V}\left(1 \pm \varepsilon_{2}\right) .
\end{aligned}
$$


For illustrative purposes, we shall assume that the error in the flowrate measurement is unimportant. It is straightforward to show that the uncertainty propogated to the mass-transfer coefficient from these two measurements is given by

$$
\begin{gathered}
\Delta^{c} \equiv \frac{k_{m}^{H}-k_{m}^{L}}{k_{m}^{A V}}=-\frac{\ln \left[\frac{1+\varepsilon_{1}}{1-\varepsilon_{1}}\right]^{2}}{\ln \left(c_{L} / c_{F}\right)^{A V}} \\
\Delta^{I} \equiv \frac{k_{m}^{H}-k_{m}^{L}}{k_{m}^{A V}}=\frac{\ln \left[\frac{1-\left(I_{c} / I_{M}\right)^{A V} \frac{1-\varepsilon_{2}}{1+\varepsilon_{1}}}{1-\left(I_{c} / I_{M}\right)^{A V} \frac{1+\varepsilon_{2}}{1-\varepsilon_{1}}}\right]}{\ln \left[1-\left(I_{c} / I_{M}\right)^{A V}\right]} .
\end{gathered}
$$

The numerator in 4 and 5 gives the difference between the highest and lowest $k_{m}$ value when the uncertainty $\varepsilon_{i}$ of the measurement is considered. The denominator normalizes the expression with respect to the average $k_{m}$. Typical values for the errors are $\varepsilon_{1} \approx 0.01$ and $\varepsilon_{2} \approx 0.005$.

Figure 7.1 is a plot of these two equations. At $\theta_{L}^{A V}=0.0149$, $\Delta^{I}$ is infinite. This graph succintly illustrates that at high conversions (low $\theta_{L}$ ) one should calculate $k_{m}$ from the concentration measurement whereas at low conversions $\left(\theta_{L} \rightarrow 1\right)$ one should calculate $k_{m}$ from the current measurement. There is a region between these two extremes where both measurements can be used but one must first arbitrarily set a value for the ordinate. This region will of course be a function of the $\varepsilon_{i}$ in each measured variable. 


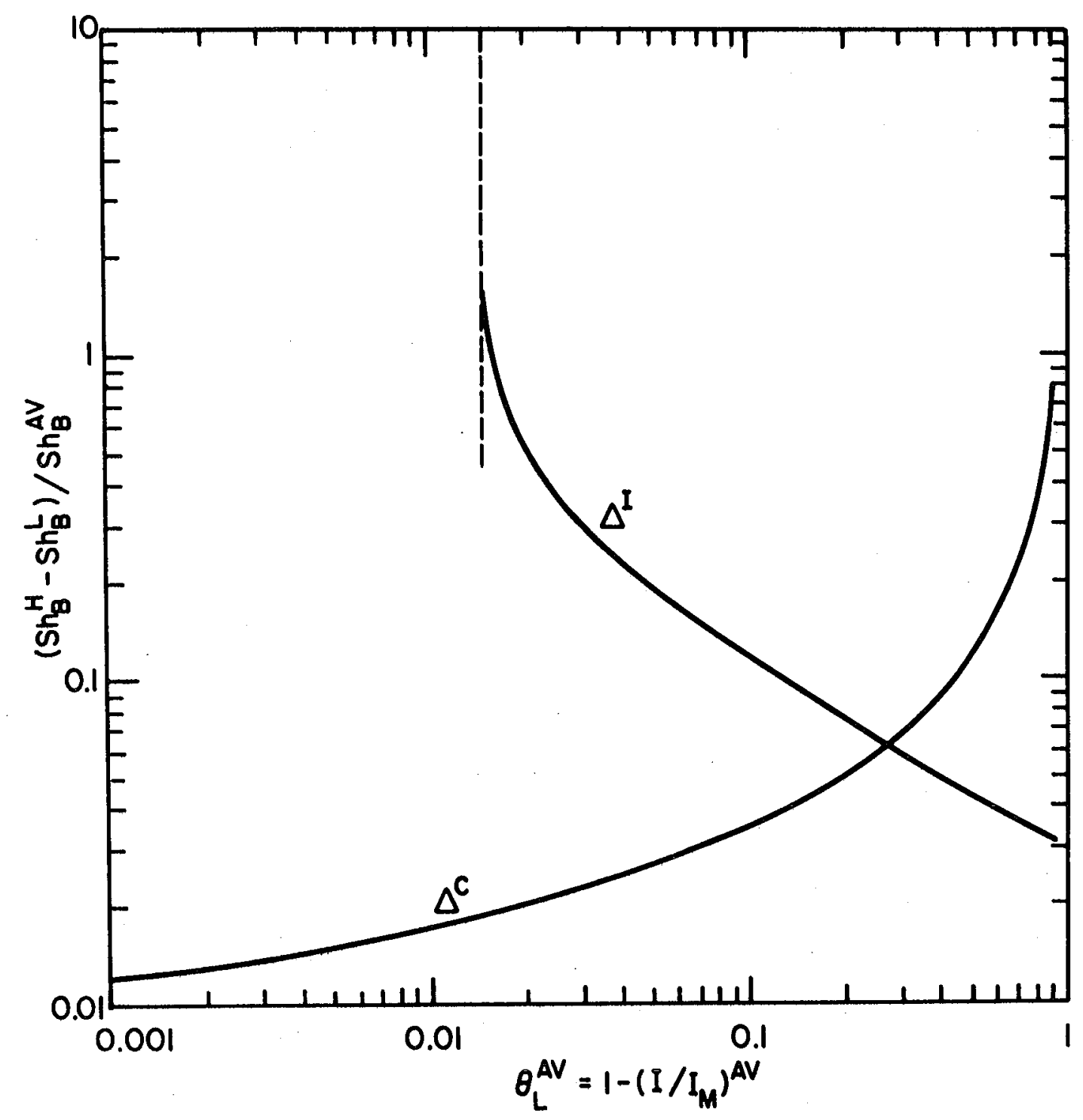

XBL 7810- 5949

Figure 7.1 Propagation of error in packed-bed mass transfer coefficient calculated from the current $\left(\Delta^{\mathrm{I}}\right)$ and the exiting concentration $\left(\Delta^{c}\right)$. The concentration is assumed to be known within $\pm 1 \%$ and the current within $\pm 0.5 \%$. 
The electrochemical technique has limitations associated with it. Because of the highly nonuniform current distribution near the limiting current, secondary (side) reactions may take place in one section of the bed while not in another. As the electrode is made more cathodic, these undesired side reactions can become significant. These reactions can eliminate the current plateau. The effect becomes pronounced as the flowrate is increased or as the concentration of the electroactive species is either made too high or too low. An elegant quantitative treatment of this problem has been given by Trainham and Newman (35, 39 ). Alkire and Gould (36) have performed similar calculations.

\section{Experimenta1 Procedures}

In the following sections, the detailed description of the cell design and procedure followed is given. The reader not interested in these details should reread the summary section in the beginning of this chapter and skip to the Results section.

\section{Choice of Test Reaction}

Selman has 1isted many of the reactions used for measuring limiting currents. The most popular seems to be the ferro-ferricyanide redox couple. This couple could not be used in these experiments because the low flowrates and the deep bed studied shift it extremely to the reduced or oxidized species. (Reduction of 3 orders of magnitude is possible.) The concentration measurement in this extreme has a 
large amount of uncertainty $\left(\varepsilon_{1}\right)$ associated with $i t$. The calculated mass-transfer coefficients would then be questionable.

The copper deposition reaction from an acidified copper sulfate solution was chosen for this study. Atomic absorption can measure the Cu concentration at $0.1 \mathrm{ppm}$ with an accuracy of \pm 1 percent. G1ycerol was added in some of the runs to increase the Schmidt number. Table 7.1 lists some advantages and disadvantages of this particular reaction. The physical properties of the solutions without glycerol were calculated from the equation of Hsueh (97). The data for $\mu, \rho$, and $D_{0}$ for the acidifled glycerol solutions were calculated from the results of Arvia et al. (98).

\section{Preparation of Packing Material for Copper Deposition}

The bed was packed with precision $1 / 8 \mathrm{in} .(3.18 \mathrm{~mm}) 316$ stainless steel bearings (Hartford Ball Co., Conn.). By the use of precision bearings, an accurate estimate for the area available for transport can be made. This should be contrasted to the dissolution of spherical benzoic acid particles where the diameter may decrease as much as $5 \%$ during the run. Also contrast this to a graphite or screen packed bed where the area must be estimated.

Preliminary runs were required to find the correct surface activation procedure so that a limiting current plateau was observed. The procedures suggested by Appel, and by Alkire and Gracon, and that found in Modern Electroplating (99) were tried. None of these was successful. It was found by trial and error that if the bearings were 
Table 7.1

Comments on the Deposition of Acidified $\mathrm{CuSO}_{4}$ Solutions as a Test Reaction for

Packed Bed Mass-Transfer Coefficients

\section{Advantages}

- well tested system; there exists a large compilation on the solution physical properties

- high exchange current insures a limiting current

- atomic absorption spectroscopy can measure $\mathrm{Cu}$ concentration to $0.100 \mathrm{ppm}$ with $\varepsilon_{1} \cong 0.01$

\section{$\underline{\text { Disadvantages }}$}

- approximately only 0.3 volt available for the $\mathrm{Cu}^{+}$deposition from a $0.01 \mathrm{M}$ solution before $\mathrm{H}_{2}$ evolution is thermodynamically possible $(\mathrm{pH} \sim 0)$

- for high $\mathrm{Cu}^{+}$concentrations, density differences during deposition can induce natural convection

- prolonged deposition times can cause surface area changes (function of current passed) 
precoated with a $\mathrm{Cu}$ plate, kinetic limitations were minimized, and the plateau could be observed. The coating procedure was as follows. The balls were acid washed, rinsed, electrolytically cleansed by anodic polarization in an acid bath, rinsed, nickel striked, $\mathrm{Cu}$ plated from a CuCN bath, rinsed, and air dried. The procedure used is that given in Metal Finishing (100). In no case was the coating greater than 1 mil thick. The bearings were in a barrel plater for a11 of the above steps. The work was done by the plating shop of the Lawrence Berkeley Laboratory.

After the plated bearings had been exposed to the atmosphere for some time, the initial bright copper was discolored due to the oxide formation. This oxide could be removed before the bearings were used by soaking them in a $1 \underline{\mathrm{M} \mathrm{H}}_{2} \mathrm{SO}_{4}$ solution.

\section{Cell Design}

The final cell design emerged as an evolutionary process. A change in the cell design was accepted or rejected on the basis of preliminary runs. The final design along with motivation for various features is presented below.

Figure 7.2 is a diagram of the cell. The cell proper consists of 4 major pieces; the cathode, anode, and head compartment all made of glass and a lucite feed ring. The anode section is attached by a spring clamp to the head compartment which is in turn bolted to the feed ring by 6 bolts attached to a collar. The cathode section is bolted to the feed ring in the same manner. 


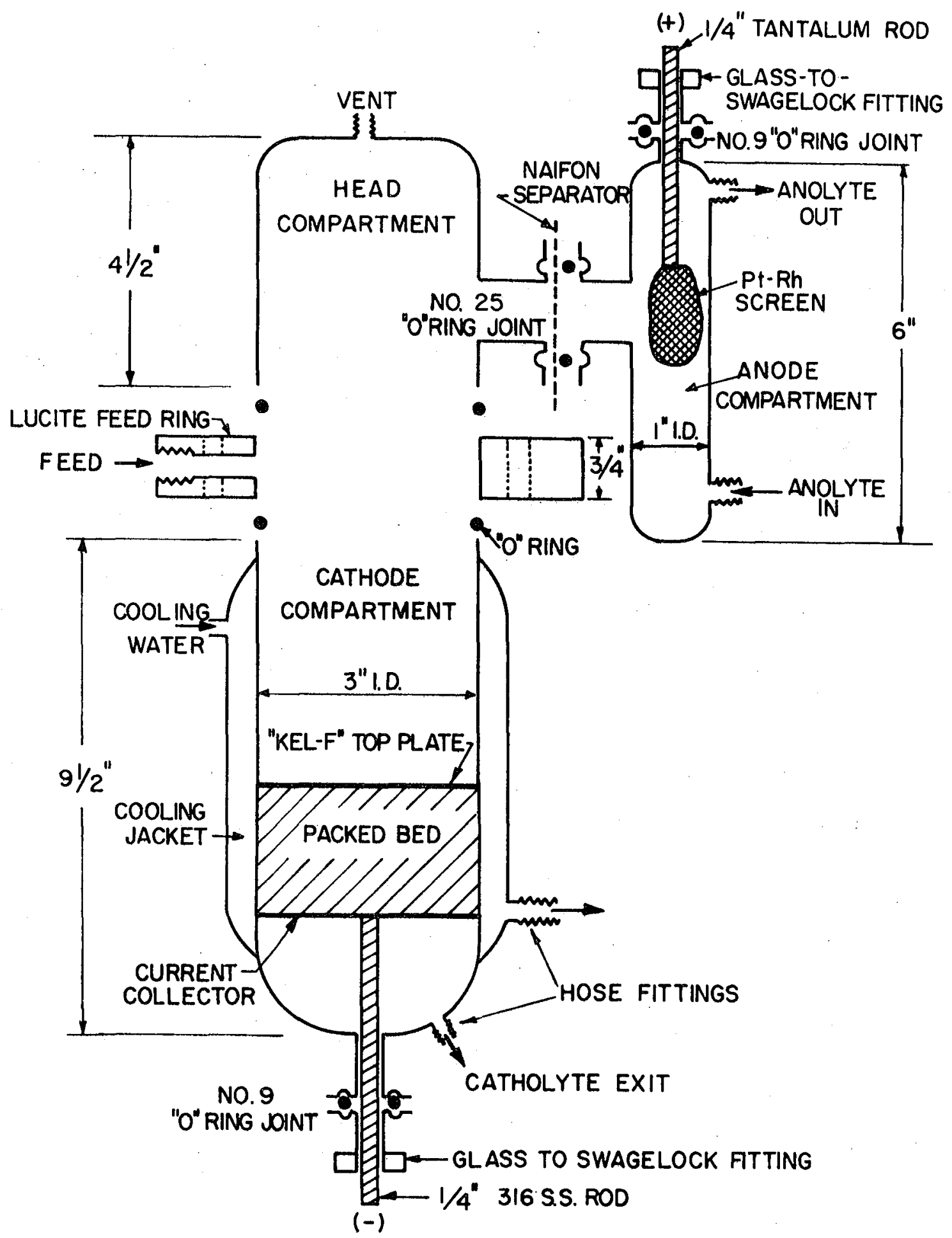

XBL7810-5951

Figure 7.2 Porous flow-through electrode. 
The reaction at the anode is $\mathrm{O}_{2}$ evolution. Because this $\mathrm{O}_{2}$ could be reduced at the cathode, it was necessary to isolate the anode from the cathode by the Naifon ${ }^{R}$ membrane (DuPont Co.), a perfluorosulfonic acid exchange membrane.

The anode consists of a Pt - Rh screen spot welded onto a $1 / 4$ in. $(6.35 \mathrm{~mm})$ tatalum rod. The screen was hung vertically so that the evolving $\mathrm{O}_{2}$ would not "stick" to the mesh and occlude the surface area. At the higher currents, the blocking effect was a problem unless this step was taken.

The head and cathode compartments were joined at the lucite feed ring. This ring had 12 equally spaced holes drilled through it. A collar with 6 bolt holes fit around each compartment. These collars were fastened by bolts at the feed ring. A seal was achieved by using "0" rings.

The bearings were supported by a 316 stainless steel current collector shown in figure 7.2 and in an isolated view in figure 7.3. The current collector was drilled with a $\#_{59} \mathrm{dril1}$ (dia $=0.0410 \mathrm{in}$. = $1.04 \mathrm{~mm}$ ) to give a matrix of holes with a surface porosity of 0.29 . The drilling was done on a microprocessor controlled press; thus the matrix was spatially homogeneous. The surface area of the current collector was $2 \%$ of the particle surface area for the shallowest pack $(a L=30)$. The back of the current collector was Kynar coated to insulate it from the solution. The plate was welded to a $1 / 4 \mathrm{in}$. diameter, 316-stainless steel rod. This rod was also Kynar coated and wrapped in Teflon tape to insure electrical insulation. Filter paper and a nylon back plate were also used as shown in Figure 7.3 . 


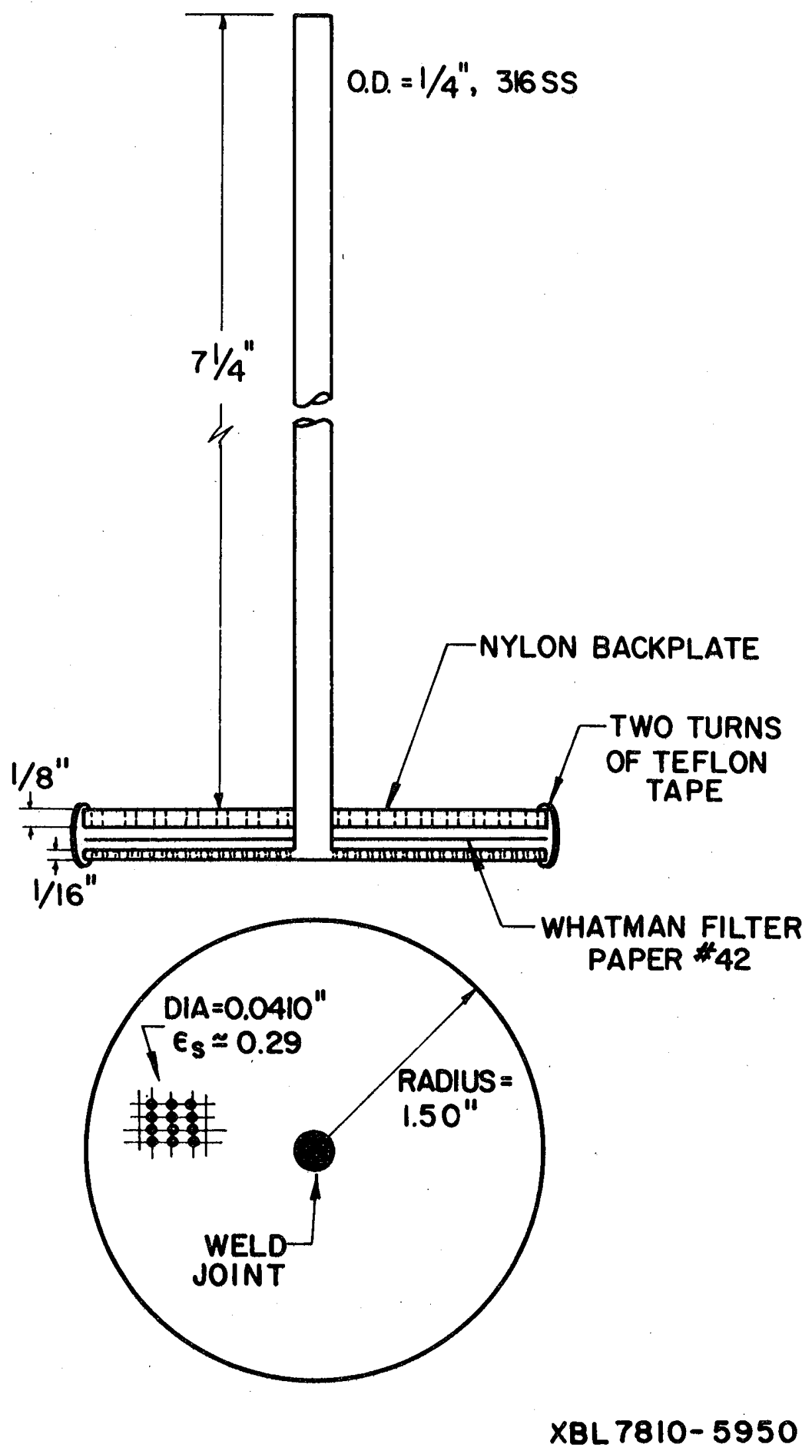

Figure 7.3 Cathode current collector. 
Fluid Flow Systems

The fluid flow system is shown in Figure 7.4. A11 connections are made by leached $1 / 4$ in. i.d. Tygon tubing.

The feed solution was pumped from its reservoir to the reactor. Before entering the reactor through a port in the feed ring, the solution passed through a glass heat exchange coil situated in the cooling water tank and then through a rotameter. The entire feed passed through the cathode. Downstream of the reactor a glass thermometer was inserted through a glass "T" joint so that the catholyte temperature could be measured. The thermometer was approximately $10 \mathrm{~cm}$ from the exit port. Downstream of the thermometer a saturated calomel reference electrode was inserted in a PVC "T" joint. The electrode was approximately $41 \mathrm{~cm}$ from the bed exit port. This distance was required because the reference electrode must be placed above the fluid level in the top compartment in order that the KC1 solution can flow out through the fiber junction.

The anolyte $\left(1 \mathrm{M} \mathrm{H}_{2} \mathrm{SO}_{4}\right)$ was continuously recirculated from a 4 1iter flask.

Fluid Metering Inc. piston metering pumps were used in the feed and anolyte lines. The piston was ceramic, and the cylinder lining was made of carbon. All parts of the pump that were in contact with the solution were electrically isolated from ground potential. This is necessary to insure that current flows only between the anode and cathode of the bed. For this same reason, no metal probes, e,g. a thermistor, should be inserted anywhere in the reaction flow system. 

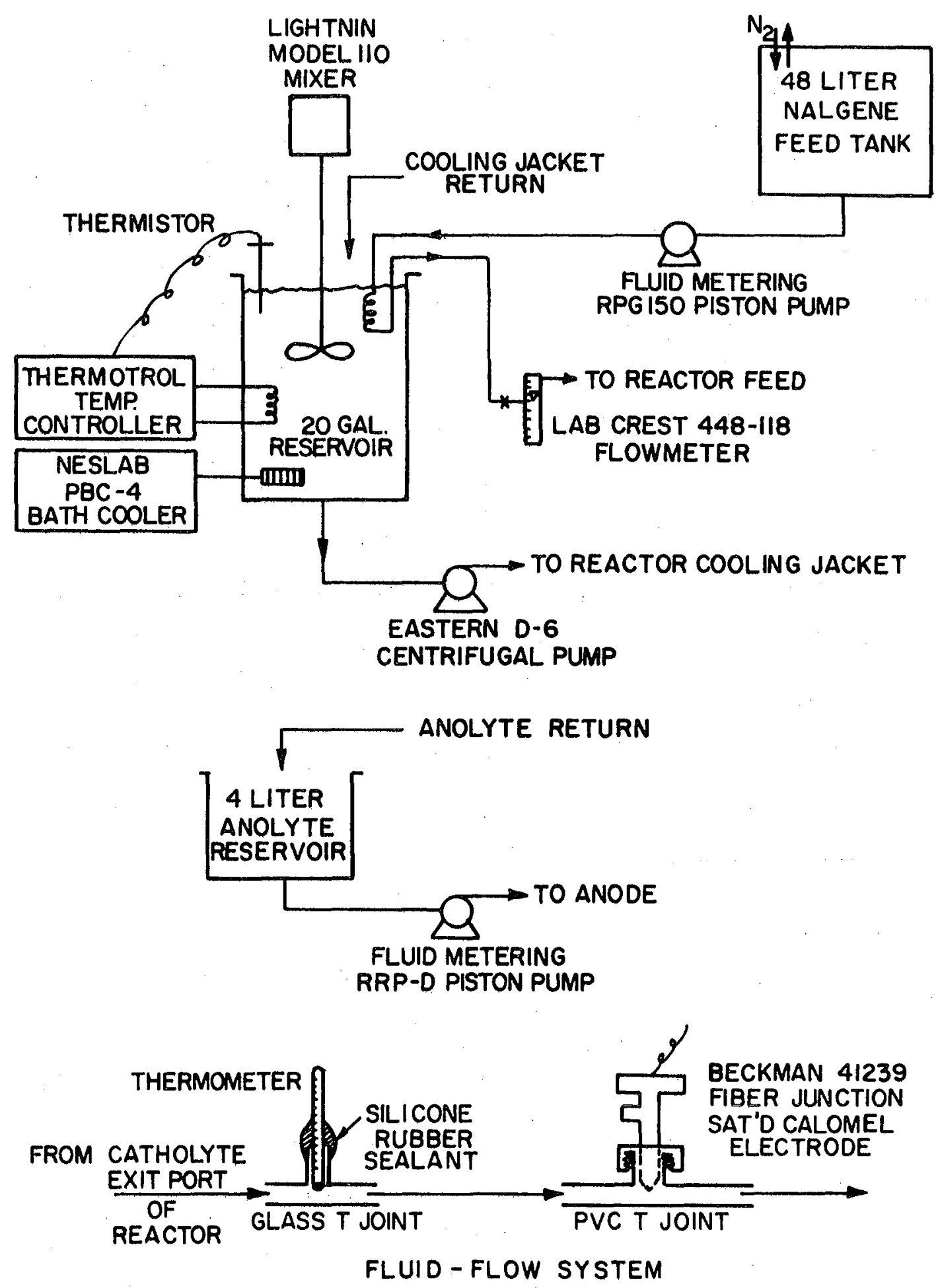

XBL7810-5954

Figure 7.4 Fluid flow system in 3 isolated views for porous electrode reactor. 
The temperature of the catholyte leaving the bed was controlled by adjusting manually the set point of the Thermotrol controller. In this manner temperature regulation of $25.0^{\circ} \mathrm{C} \pm 0.2^{\circ} \mathrm{C}$ (with some excursions as high as $\pm 0.5^{\circ} \mathrm{C}$ ) could be maintained.

\section{Electrical Circuit}

Two methods were used to apply a potential difference across the cell. One technique, the power supply mode, is shown in Figure 7.5. This is the method used by Bennion and Newman to power their cell. In this circuit the overall cell potential $V_{A}-V_{C}$ is set. The cell current and the polarization of each electrode relative to the calomel reference electrode will adjust themselves accordingly. The other mode used was potentiostatic control of the cathode with respect to the saturated calomel reference electrode in the catholyte. The circuitry for this method is diagrammed in Figure 7.6. This was the control mode used by Appe1. The majority of the runs were carried out in the potentiostatic control mode.

Preliminary runs were carried out with potentiostatic control without the $2 \mu \mathrm{F}$ capacitor inserted between the anode and reference electrode. In the course of these runs it was discovered that the cell current was rapidly, periodically fluctuating as much as $10 \%$ about a mean. This was clearly seen when the potential drop across the $1 \Omega$ resistor was examined on an oscilloscope. The reason for this behavior is clear upon reflection. The potentiostat is a high gain proportional controller. If there exists a large enough 


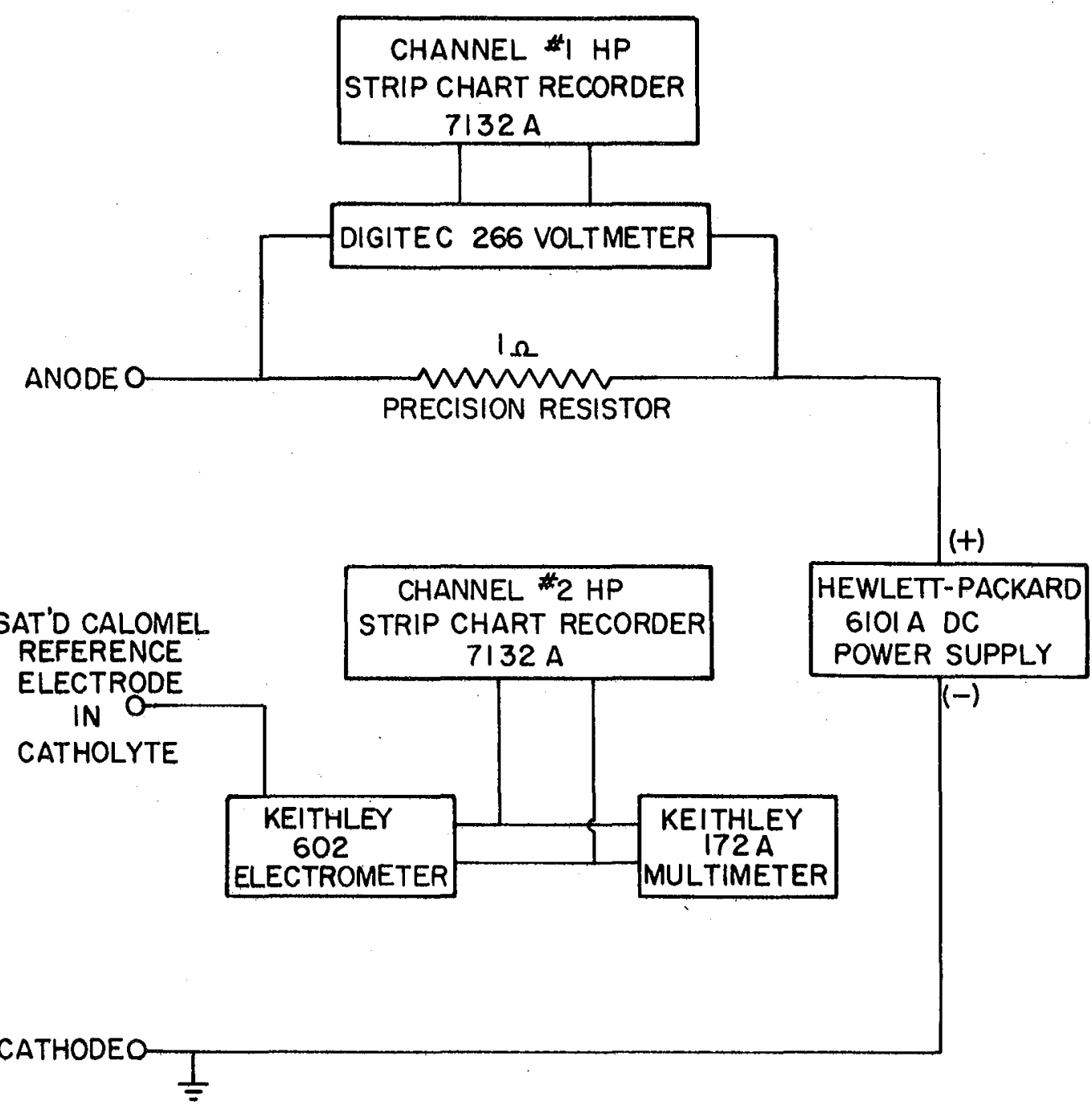

Figure 7.5 ELECTRICAL CIRCUIT - POWER SUPPLY MODE

XBL 7810-5953 


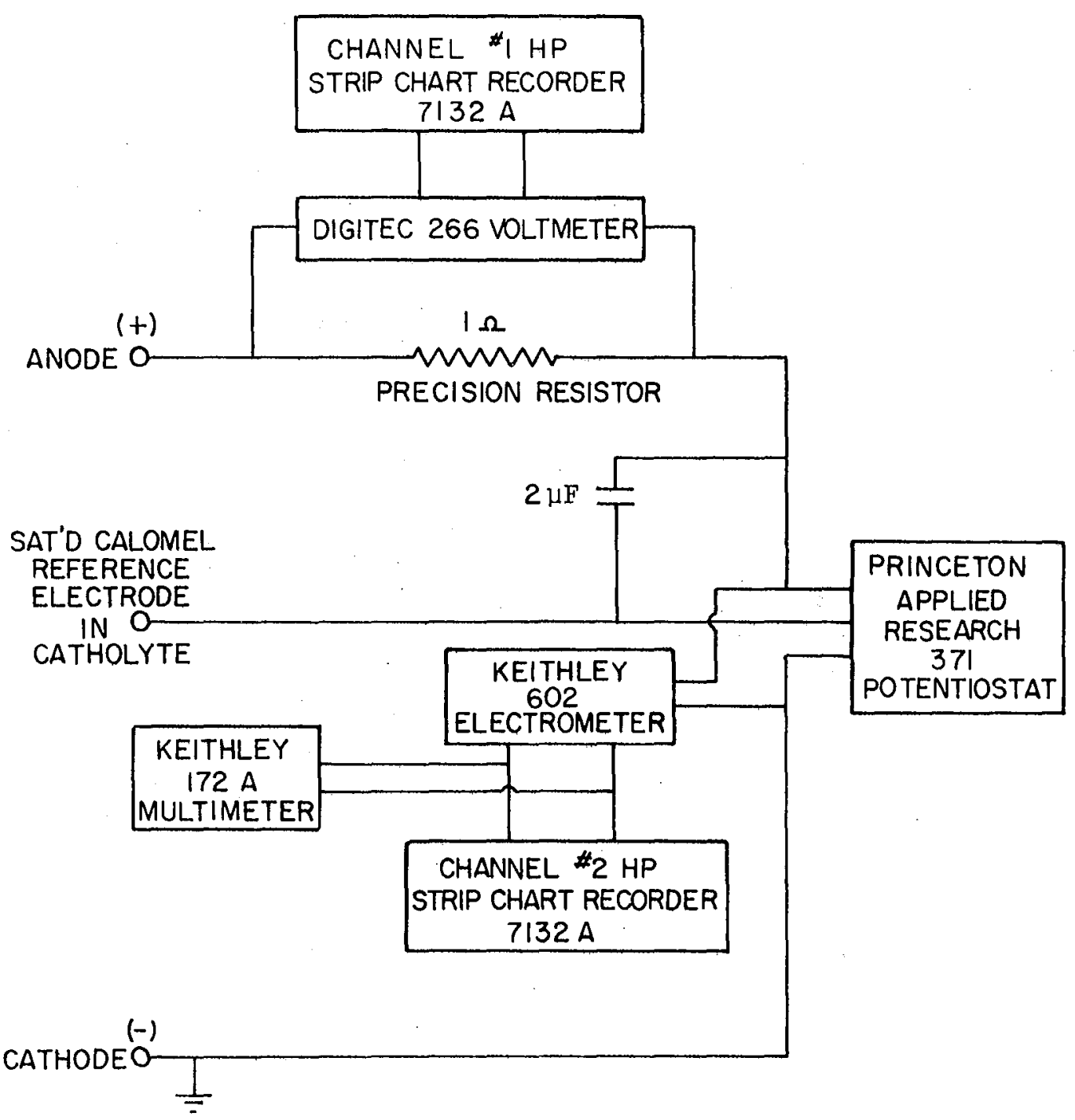

Figure 7.6 ELECTRICAL CIRCUIT - POTENTIOSTATIC CONTROL MODE 
capacitance between the cathode and the anode, the potentiostat can be forced into oscillations. The capacitor inserted between the anode and reference electrode damps out these oscillations. With the capacitor inserted, no trace of the oscillations could be seen on an oscilloscope. For further discussion on electrochemical cell control the reader is referred to Harrar and Pomernacki (101) and Schroeder and Shain (102).

[The Information contained in the oscillations could be constructively used. If the frequency and amplitude of the current oscillations can be measured, one may calculate the effective resistance and capacitance for the porous electrode. The transfer function of the potentiostat and reference electrode need only be known.]

\section{Preparation of Electrolytic Solutions}

Al1 chemicals used were AR grade. The acidified copper sulfate solutions were made in $48 \mathrm{l}$ batches. The water used was first distilled and then run through a Culligan SR cartridge water system. The specific resistivity of this water was 10-15 meg ohm $-\mathrm{cm}$. The acid was added to the water, mixed, and then the $\mathrm{CuSO}_{4}$ was added in predissolved form. After a mixing period, $\mathrm{N}_{2}$ gas was bubbled through the solution. In al1 cases at least 10 hours of this de-oxygenation procedure were allowed before the solution was used.

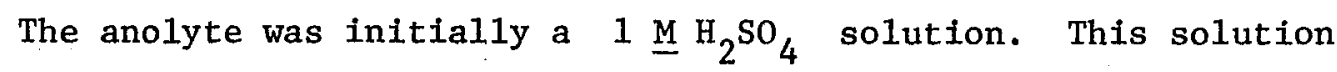
becomes more acidic in the course of the cell lifetime because of the $\mathrm{O}_{2}$ evolution reaction. It was changed every 10 runs. 
The solutions with glycerol were made up identically as those without. The glycerol was added after the $\mathrm{CuSO}_{4}$. These solutions were contained in a 26 \& feed tank.

A blanket of $\mathrm{N}_{2}$ gas was kept over the feed solution in the course of the run.

The concentration of the species were $\mathrm{CuSO}_{4}: 0.001$ to $0.01 \mathrm{M}$; $\mathrm{H}_{2} \mathrm{SO}_{4}: 1$ and $1.5 \underline{\mathrm{M}} ; \mathrm{C}_{3} \mathrm{H}_{5} \mathrm{O}_{3}: 0$ and $3 \underline{\mathrm{M}}$. The $\mathrm{Cu}$ concentration was measured by atomic absorption spectrophotometry. The acid was determined by titration with standard $\mathrm{NaOH}$ with methyl red as the indicator. The glycerol content was determined by ceric oxidation following the procedure of Smith and Duke (103).

\section{Procedure for a Run}

The cathode compartment was placed in a specially designed holder for mechanical support. The cell was cleansed with copious amounts of distilled water. A $1 / 4 \mathrm{In}$. rod was placed through the Swagelock fitting on the bottom of the cathode compartment. The catholyte exit port was closed off by a hose clamp, and the cell was partly filled with distilled water. Glass spheres $1 / 8$ in. in diameter were then dropped into the cathode compartment. These spheres serve to decrease the lag time between the fluid exiting the back of the current collector and passing the reference electrode. The current collector was assembled and then inserted into the cathode compartment with simultaneous withdrawal of the $1 / 4 \mathrm{in}$, rod which was initially in the port. At the end of this procedure, the current collector rests submersed 
upon the top layer of the glass spheres in the position depicted in Figure 7.2. The bed is now prepared to pack with the bearings.

The correct amount of $\mathrm{Cu}$ plated bearings to obtain a certain packing depth was determined by weight. The average weight per bearing was determined over a large sample size. The total number of bearings required to pack the bed to a specified al could then be calculated by assuming a value for the porosity. In this manner, with an $\varepsilon$ of 0.38 , approximately 4300 balls are needed for an $\mathrm{aL}=30$, and 14,400 are needed for an $a L=100$.

The packing of the bed proceeds as follows. Enough distilled water was added to the cell so that the particles are always submerged. In this manner air pockets could not be formed in the intersticies of the packing. The bearings were poured from a small beaker into the bed. When the incremental height of these bearings was $1 / 2 \mathrm{~cm}$, a tamper, 3 in. in diameter, connected to a 1/4 in. rod was centered over the bearings and was slowly rotated, and the bed was simultaneously compressed. In this manner the layer was fairly uniform and tightly packed. This packing-tamping procedure was carried out until all of the preweighed bearings were used. As a final step, a 3 in. diameter, 1/16 in. thick piece of $\mathrm{Kel}-\mathrm{F}$, which was drilled with the same hole matrix as the current collector, was tightly pressed on top of the spheres. A piece of Whatman $\$ 42$ filter paper was held on the top side of this plate by four turns of Teflon tape. This filter paper/plate combination helped minimize contact resistance between the bearings, and it also acted as a flow distributor. It also removed suspended matter from the 
electrolyte. Early runs showed that the same results were obtained when glass spheres were added on top of the plate as when they were not. For the majority of runs, no top layer of spheres was used. The height of the packing was measured with a cathetometer at four equally spaced angular positions. If the standard deviation about the average packing depth exceeded 3 pexcent of the average, the bed was tamped down, and another set of measurements was taken.

After the height measurements were completed, the bed was drained to $1 \mathrm{in}$. above the Kel-F plate. Feed solution was then added up to the feed ring. The cell was then assembled. Electrical and flow connections were then made.

The following discussion is specifically directed to the potentiostatic control mode.

A low cathodic polarization was applied to the bed while the feed solution flushed out the remaining distilled water. After 1 liter of solution had passed through the ce11, a low polarization $(-50 \mathrm{mV})$ was applied to the cathode, and the cell was allowed to operate overnight at a very low flowrate $(<1 \mathrm{ml} / \mathrm{min})$.

After this treatment data collection could proceed. A control potential was selected on the potentiostat, a flowrate was set, and, after 3 residence times had passed, effluent samples were collected. At the same time the flowrate was determined by measuring the flow to a calibrated cylinder. The potential was then made more cathodic by slowly $(\sim 1 \mathrm{mV} / \mathrm{s})$ increasing the potentiostat control setting. By following this procedure an entire polarization curve could be mapped 
out. The first polarization applied was usually -90 to $-100 \mathrm{mV}$. The current at this potential was nearly the limiting value at the lower flowrates studied. It was empirically determined in early runs that $\mathrm{H}_{2}$ evolution would begin in the range of -270 to $-320 \mathrm{mV}$. If care was taken to avoid $\mathrm{H}_{2}$ evolution, the same pack could be used at a different flowrate. When there was evidence of $\mathrm{H}_{2}$ evolution, the run was terminated. The $\mathrm{H}_{2}$ could be visually observed as void spaces near the top of the bed. A more sensitive probe was the current. At high cathodic potentials the current would start to fluctuate. It is speculated that the $\mathrm{H}_{2}$ gas formation causes this fluctuation.

The data measured were the applied polarization $\left(V_{C}-\phi_{R}\right)$, the overa11 cell voltage $\left(V_{A}-V_{C}\right)$, the cell current $\left(I_{C}\right)$, the flowrate (Q) , and the catholyte temperature $\left(T_{E}\right)$.

\section{$\underline{\text { Results }}$}

A total of 83 runs were made. A run is defined as the measurement of a polarization curve at a specified flowrate. Of these 83 runs, 59 mass transfer data points were retained. The remainder of the runs were rejected because the two coefficients calculated from the current and effluent concentrations deviated by more than 10 percent. of these 59 points, 33 were taken in a bed with aL $\approx 30$, and 26 were from a bed with aL $\approx 100$; furthermore, 9 of the aL $\approx 30$ points were taken with glycerol added to the feed.

The data and some preliminary calculations for the 59 runs are listed in tabular form in appendix D. 
Table 7.2 lists the calculated results. The average deviation for the Sherwood number is at the most \pm 5 percent. These results are plotted as $S h_{B}$ vs $\mathrm{Pe}_{\mathrm{B}}$ in Figure 7.7. The results of Appel with $a L \simeq 10$ are also included

Figure 7.8 illustrates where the data collected in this present study are situated with respect to other published works. 
Table 7.2

Calculated Results

\begin{tabular}{|c|c|c|c|c|c|c|c|c|}
\hline Run & $\varepsilon$ & $\mathrm{aL}$ & $\frac{{ }^{\mathrm{d}} \mathrm{p}}{v}$ & $\frac{{ }^{v d}{ }_{p}}{\varepsilon v}$ & $\frac{\mathrm{v}}{\mathrm{av}}$ & $\frac{v}{D_{0}}$ & $\frac{\mathrm{v}}{\mathrm{a} \mathrm{D}_{\mathrm{o}}}$ & $\frac{\varepsilon \mathrm{k}_{\mathrm{m}}}{\mathrm{a} D_{\mathrm{o}}}$ \\
\hline 10 & 0.366 & 30.1 & 0.213 & 0.582 & 0.0560 & 1900 & 107 & 1.69 \\
\hline 11 & 0.391 & 29.0 & 0.409 & 1.05 & 0.112 & 1900 & 213 & 2.42 \\
\hline 12 & 0.382 & 30.4 & 0.211 & 0.551 & 0.0568 & 1910 & 108 & 1.71 \\
\hline 15 & 0.372 & 100 & 0.213 & 0.572 & 0.0565 & 1910 & 108 & 1.54 \\
\hline 18 & 0.385 & 30.4 & 0.413 & 1.07 & 0.112 & 1887 & 211 & 2.50 \\
\hline 19 & 0.374 & 30.3 & 0.413 & 1.10 & 0.110 & 1887 & 207 & 2.18 \\
\hline 20 & 0.385 & 100 & 0.413 & 1.07 & 0.112 & 1887 & 211 & 2.11 \\
\hline 21 & 0.406 & 30.3 & 0.0880 & 0.217 & 0.0247 & 1887 & 46.5 & 1.34 \\
\hline 22 & 0.390 & 100 & 0.0878 & 0.225 & 0.0240 & 1903 & 45.9 & 1.04 \\
\hline 24 & 0.390 & 29.1 & 0.125 & 0.320 & 0.0341 & 1903 & 65.0 & 1.48 \\
\hline 25 & 0.393 & 29.7 & 0.0477 & 0.121 & 0.0131 & 1910 & 25.1 & 0.828 \\
\hline 27 & 0.385 & 100 & 0.0450 & 0.117 & 0.0122 & 1894 & 23.2 & 0.477 \\
\hline 28 & 0.385 & 100 & 0.0664 & 0.173 & 0.0180 & 1894 & 34.0 & 0.642 \\
\hline 30 & 0.394 & 29.7 & 0.0436 & 0.111 & 0.0120 & 1894 & 22.8 & 0.787 \\
\hline 31 & 0.392 & 30.4 & 0.165 & 0.422 & 0.0453 & 1921 & 87.1 & 1.80 \\
\hline 32 & 0.396 & 30.5 & 0.0249 & 0.0628 & $6.83 \times 10^{-3}$ & 1906 & 13.1 & 0.615 \\
\hline 33 & 0.396 & 30.5 & $9.82 \times 10^{-3}$ & 0.0248 & $2.71 \times 10^{-3}$ & 1906 & 5.20 & 0.330 \\
\hline 34 & 0.396 & 30.5 & 0.137 & 0.345 & 0.0377 & 1906 & 71.8 & 2.07 \\
\hline 35 & 0.392 & 101 & 0.0290 & 0.0741 & $7.96 \times 10^{-3}$ & 1906 & 15.2 & 0.450 \\
\hline 36 & 0.392 & 101 & 0.0522 & 0.133 & 0.0143 & 1906 & 27.2 & 0.683 \\
\hline 37 & 0.392 & 101 & 0.169 & 0.431 & 0.0463 & 1906 & 88.2 & 1.50 \\
\hline 40 & 0.400 & 30.4 & 0.0695 & 0.174 & 0.0193 & 1910 & 36.8 & 1.08 \\
\hline 41 & 0.393 & 99.9 & 0.124 & 0.315 & 0.0340 & 1910 & 64.9 & 1.41 \\
\hline 42 & 0.393 & 99.9 & 0.0503 & 0.128 & 0.0138 & 1910 & 26.5 & 0.897 \\
\hline 43 & 0.393 & 99.9 & 0.104 & 0.265 & 0.0286 & 1910 & 54.6 & 1.26 \\
\hline 44 & 0.393 & 99.9 & 0.185 & 0.471 & 0.0508 & 1910 & 97.1 & 1.78 \\
\hline 45 & 0.393 & 99.9 & 0.230 & 0.585 & 0.0631 & 1910 & 121 & 2.10 \\
\hline 47 & 0.393 & 99.9 & 0.341 & 0.725 & 0.0937 & 1910 & 179 & 2.55 \\
\hline 48 & 0.393 & 99.9 & 0.390 & 0.868 & 0.107 & 1910 & 204 & 2.75 \\
\hline 51 & 0.373 & 30.3 & 0.489 & 1.31 & 0.130 & 1915 & 249 & 2.86 \\
\hline
\end{tabular}




\section{Table 7.2 (continued)}

Calculated Results

Run $\varepsilon \quad$ aL $\quad \frac{v d}{\nu} \quad \frac{v d p}{\varepsilon v} \quad \frac{v}{a v} \quad \frac{v}{D_{0}} \quad \frac{v}{a D_{0}} \quad \frac{\varepsilon k m}{a D_{0}}$

\begin{tabular}{lllllllll}
\hline 52 & 0.373 & 30.3 & 0.564 & 1.51 & 0.150 & 1915 & 288 & 3.07 \\
53 & 0.373 & 30.3 & 0.673 & 1.81 & 0.179 & 1915 & 343 & 3.22 \\
55 & 0.383 & 100 & 0.288 & 0.753 & 0.0779 & 1926 & 150 & 1.99 \\
56 & 0.383 & 100 & 0.515 & 1.34 & 0.139 & 1926 & 267 & 2.57 \\
57 & 0.383 & 100 & 0.418 & 1.09 & 0.113 & 1926 & 218 & 2.41 \\
58 & 0.383 & 100 & 0.581 & 1.52 & 0.157 & 1926 & 302 & 2.87 \\
59 & 0.383 & 100 & 0.773 & 1.91 & 0.198 & 1926 & 382 & 3.33
\end{tabular}

$\begin{array}{lllllllll}61 & 0.400 & 29.9 & 0.0580 & 0.145 & 0.0161 & 1919 & 30.9 & 1.14\end{array}$

$\begin{array}{lllllllll}62 & 0.400 & 29.9 & 0.0162 & 0.0405 & 4.50 \times 10^{-3} & 1919 & 8.63 & 0.523\end{array}$

$\begin{array}{lllllllll}63 & 0.400 & 29.9 & 0.0788 & 0.197 & 0.0219 & 1919 & 41.9 & 1.56\end{array}$

$\begin{array}{llllllllll}64 & 0.400 & 29.9 & 0.0343 & 0.0859 & 9.54 \times 10^{-3} & 1919 & 18.3 & 0.935\end{array}$

$\begin{array}{lllllllll}65 & 0.400 & 29.9 & 0.141 & 0.354 & 0.0393 & 1919 & 75.3 & 2.10\end{array}$

$\begin{array}{lllllllll}66 & 0.400 & 29.9 & 0.209 & 0.523 & 0.0581 & 1919 & 111 & 2.53\end{array}$

$\begin{array}{lllllllll}67 & 0.387 & 100 & 0.0345 & 0.0891 & 9.37 \times 10^{-3} & 1919 & 18.0 & 0.484\end{array}$

$\begin{array}{lllllllll}68 & 0.387 & 100 & 0.0222 & 0.0573 & 6.03 \times 10^{-3} & 1919 & 11.6 & 0.332\end{array}$

$\begin{array}{lllllllll}69 & 0.387 & 100 & 0.0684 & 0.177 & 0.0186 & 1919 & 35.8 & 0.770\end{array}$

$\begin{array}{lllllllll}70 & 0.387 & 100 & 0.0953 & 0.246 & 0.0259 & 1919 & 49.7 & 1.08\end{array}$

$\begin{array}{lllllllll}71 & 0.387 & 100 & 0.141 & 0.365 & 0.0384 & 1919 & 73.7 & 1.34\end{array}$

$\begin{array}{lllllllll}72 & 0.387 & 100 & 0.0817 & 0.211 & 0.0222 & 1919 & 42.5 & 0.983\end{array}$

$\begin{array}{lllllllll}74 & 0.388 & 30.0 & 0.0210 & 0.0542 & 5.73 \times 10^{-3} & 8880 & 50.9 & 1.55\end{array}$

$\begin{array}{lllllllll}75 & 0.388 & 30.0 & 8.92 \times 10^{-3} & 0.0230 & 2.43 \times 10^{-3} & 8880 & 21.5 & 0.949\end{array}$

$\begin{array}{lllllllll}76 & 0.388 & 30.0 & 0.0299 & 0.0769 & 8.13 \times 10^{-3} & 8800 & 72.1 & 1.85\end{array}$

$\begin{array}{lllllllll}77 & 0.388 & 30.0 & 0.0397 & 0.102 & 0.0108 & 8880 & 96.1 & 2.02\end{array}$

$\begin{array}{lllllllll}78 & 0.388 & 30.0 & 0.0485 & 0.125 & 0.0132 & 8880 & 117 & 2.25\end{array}$

$\begin{array}{lllllllll}79 & 0.388 & 30.0 & 0.0610 & 0.157 & 0.0166 & 8880 & 148 & 2.47\end{array}$

$\begin{array}{lllllllll}80 & 0.388 & 30.0 & 0.0782 & 0.202 & 0.0213 & 8880 & 189 & 2.88\end{array}$

$\begin{array}{lllllllll}81 & 0.388 & 30.0 & 0.102 & 0.264 & 0.0279 & 8880 & 248 & 3.21\end{array}$

$\begin{array}{lllllllll}82 & 0.388 & 30.0 & 0.133 & 0.344 & 0.0363 & 8880 & 322 & 3.57\end{array}$

$\begin{array}{lllllllll}83 & 0.388 & 30.0 & 0.174 & 0.449 & 0.0474 & 8880 & 421 & 4.08\end{array}$ 


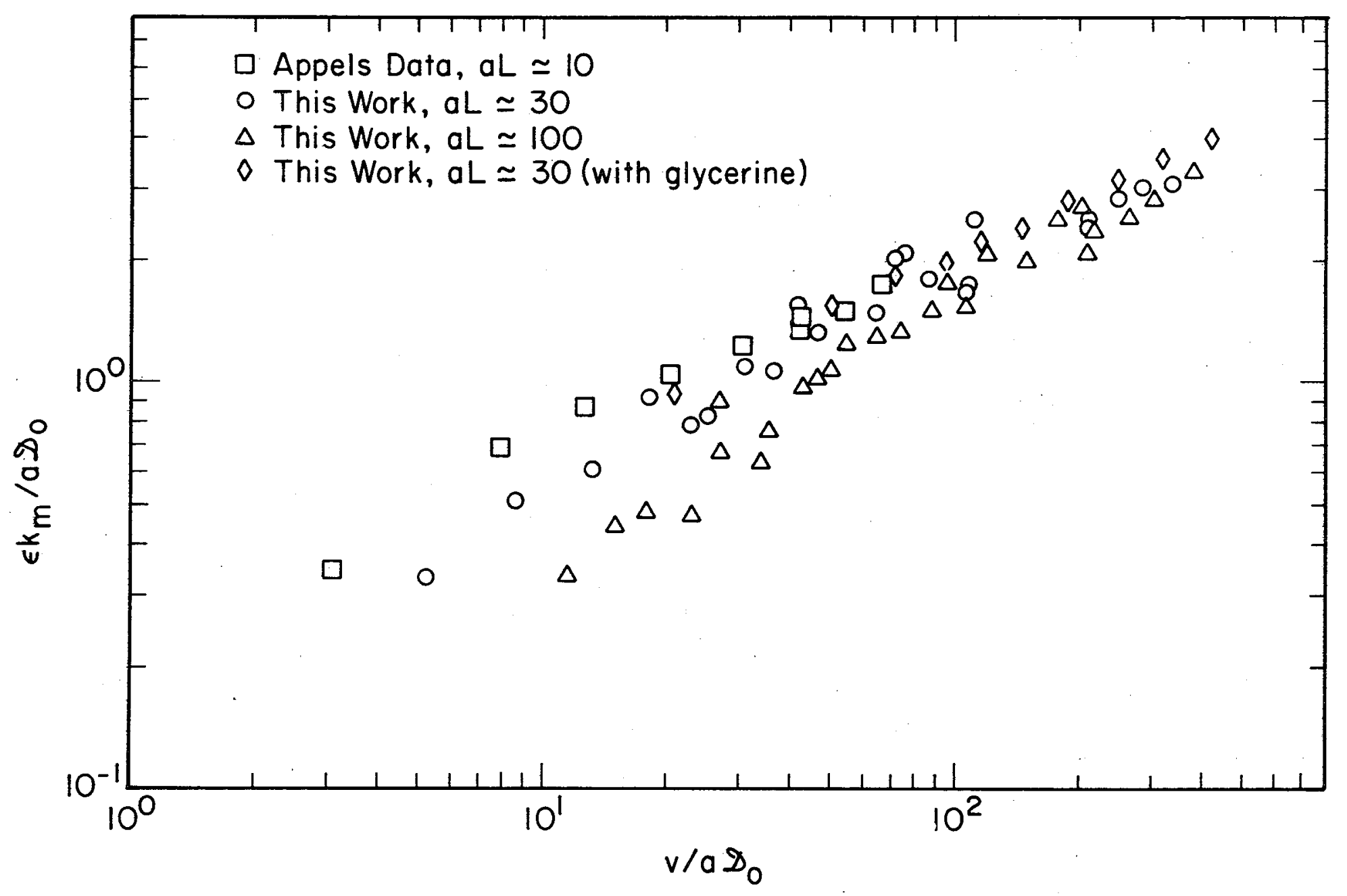

XBL 7811-12877

Figure 7.7 Mass-transfer limited Sherwood numbers for packed beds collected in this work. Appel's data are also included. 


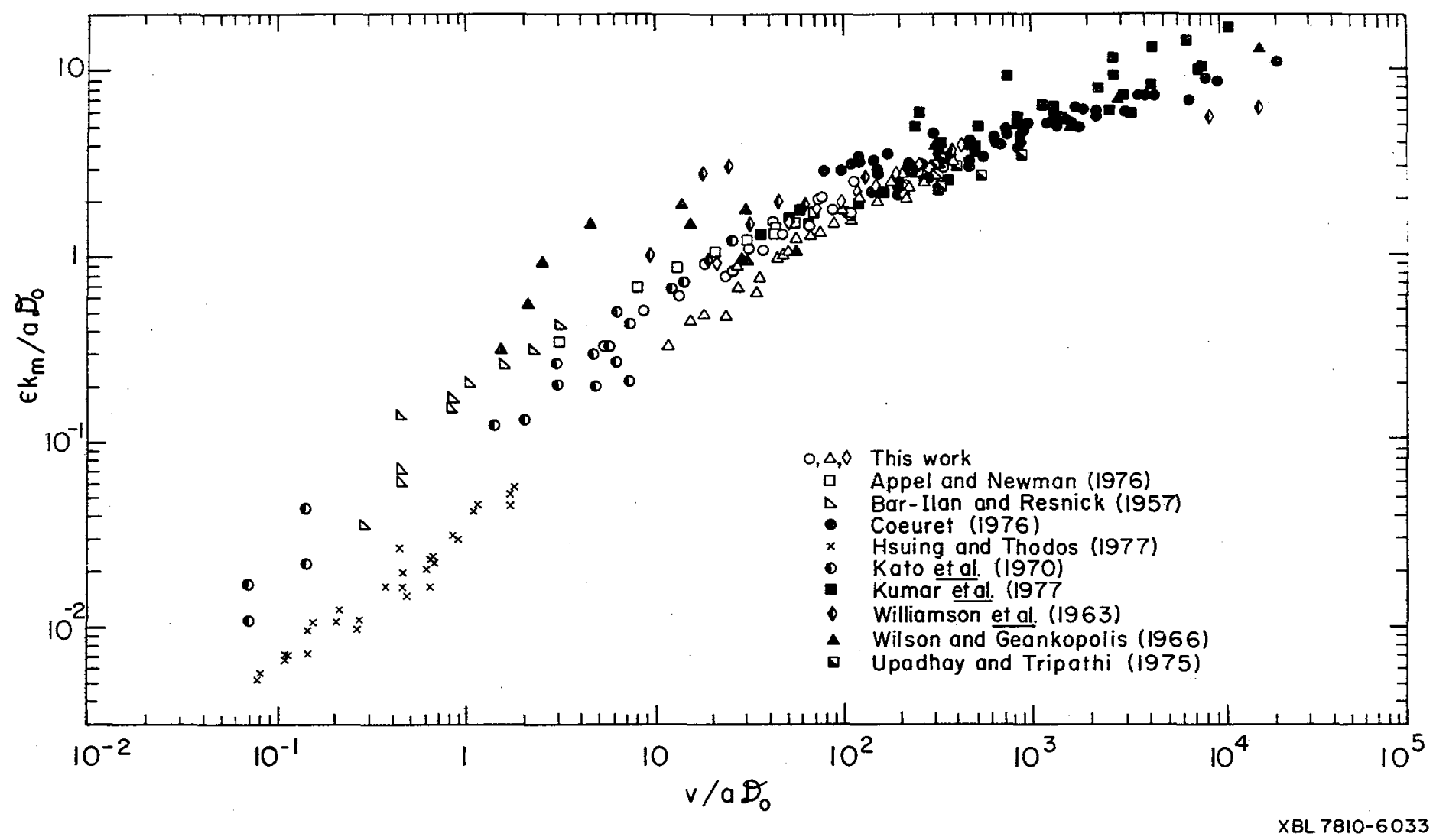

Figure 7.8 Compilation of mass-transfer limited Sherwood numbers in packed beds. The Reynolds number $(\mathrm{v} / \mathrm{a} \nu$ ) is less than one for a11 points. 
Chapter 8

Interpretation of Results

Summary

In this chapter the experimental data for mass-transport limited Sherwood numbers presented in Chapter 7 will be compared with the PCT model calculations. Different empirical combinations of the asymptotic mass-transfer coefficients are attempted in order to fit the data. The best set of unknown empirical constants are determined in a least squares sense. Each of these data fitting attempts is presented, none of which was successful. The inability of the model to fit the data enables one to learn about the deficiencies of the model. The bed was envisioned as an array of sinusoidal PCT of a single size. If the existence of multiple width channel paths is recognized, the data can be adequately fit by modelling the bed as an array of dual sized straight tubes. The complexities of the PCT model are not found useful in interpreting the data.

Combination of Asymptotes

In the earlier chapters calculations were presented for the PCT modeled, packed bed mass-transfer coefficient under different limiting conditions.

It would be highly convenient to combine these asymptotes smoothly in some manner to cover the non-asymptotic regions. Churchill and Usagi (104), expanding upon an idea suggested by Acrivos (105), have pointed out a manner to combine asymptotic formulae. Their results will be utilized here. 
Define the following quantities.

$\mathrm{Sh}_{\mathrm{B}}^{(1)}=$ Low Péclet number, deep bed asymptotic Sherwood number

$\mathrm{Sh}_{\mathrm{B}}^{(2)}=$ High Péc1et number, deep bed asymptotic Sherwood number

$\mathrm{Sh}_{\mathrm{B}}^{(3)}=\underset{\text { number }}{\text { High Péclet number, entrance region asymptotic Sherwood }}$

$\mathrm{Sh}_{\mathrm{B}}^{(4)}=$ High Péclet number, mixing region Sherwood number .

Each asymptote has been calculated in chapters 5, 3, 6 and 6 respectively.

The manner in which these asymptotes are combined is dependent

upon the physical picture one envisions for the processes taking

place within the interstices of the packing.

As a first attempt, the model of an array of PCT logically leads

one to write

$$
\frac{1}{S h_{B}}=\left\{\left(\frac{1}{S h_{B}^{(1)}}\right)^{n}+\left(\frac{1}{S h_{B}^{(2)}+S_{B}^{(3)}}\right)^{n}\right\}^{1 / n} .
$$

This is not a unique representation, but it is the simplest. The exponent $n$ must be determined by a data fitting procedure.

The usefulness of combining the asymptotic formula as suggested by equation 1 can be found by testing it with Sфrensen and Stewart's (12) calculations. These authors have solved the creeping flow hydrodynamics and convective diffusion equation in an array of uniformly sized, simple cubic packed spheres. They presented in tabular form numerical calculations for $k_{m}$ as a function of bed depth and Péclet number. They also presented formula for the $S_{B}^{(i)}$, $i=1,2,3$. Figure 8.1 is a plot of the numerically calculated $k_{m}$ 
Figure 8.1 Comparison of Sфrensen and Stewarts' numerically calculated Sherwood numbers with that given by combination of the asymptotes according to equation 1 with $n=1$.

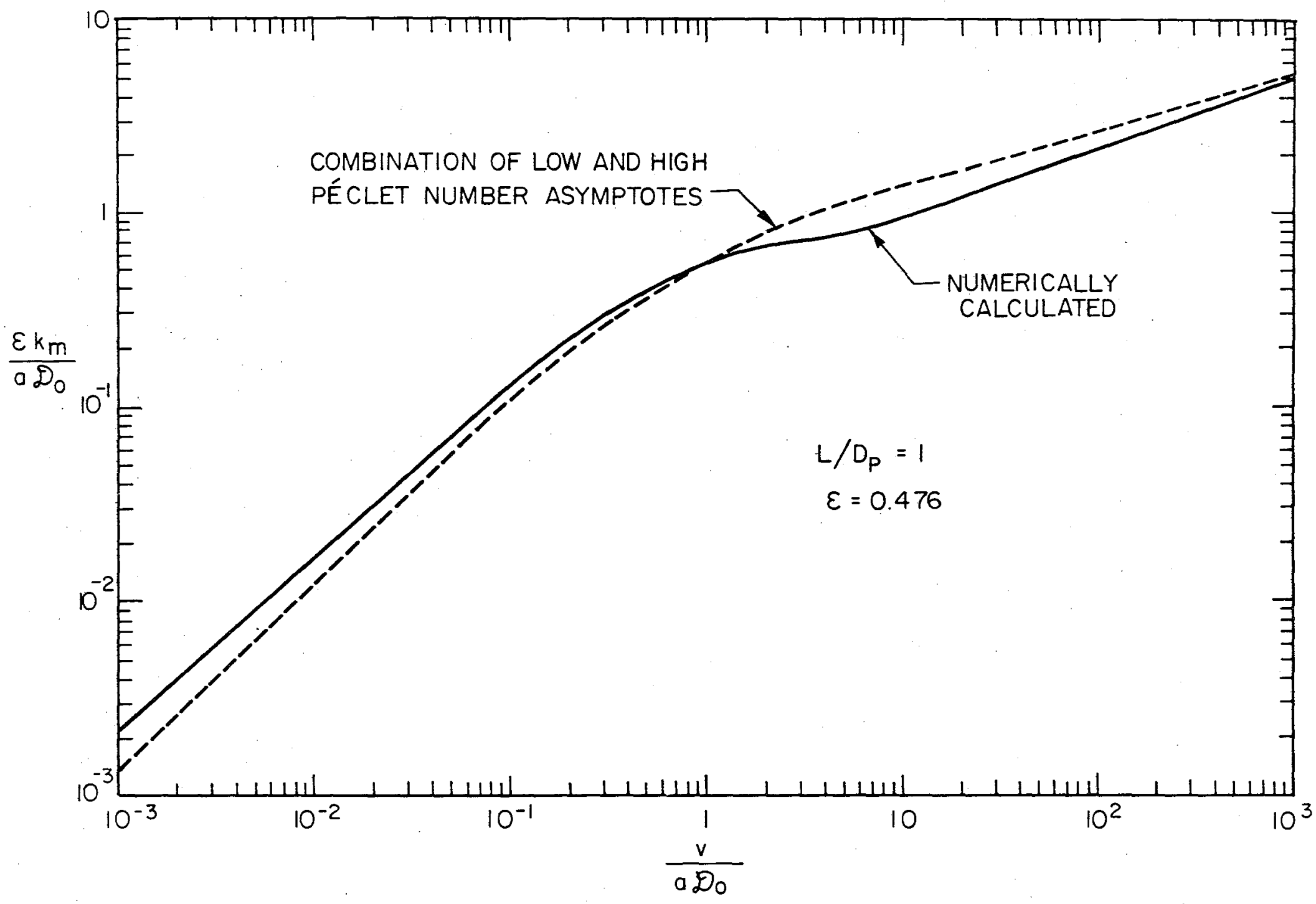


compared with that given by combination of the asymptotes according to equation 1 with $n=1$. The numerical coefficients are for a bed one particle layer deep. At low Péclet numbers, this will give a comparison for the worse discrepancy between the actual value and the deep bed asymptote which is used in equation 1 . Even in this worse case the deviation is acceptable. This is a restatement of the fact that the leading term $\alpha_{1}$, of equation 14, Chapter 4, need on1y be known for most practical sized beds.

The value of $n$ was determined by fitting the 68 data points shown in figure 7.7 to equation 1 by using a nonlinear least squares library routine (Univ. of Calif., LSQMIN), The fitted value depended upon the tube parameters used. Figures 8.2 and 8.3 are plots of equation 1 with $\mathrm{n}=0.42$ and $\mathrm{n}=0.50$; Figure 8.2 is plotted for a sinusoidal PCT with $\mathrm{r}_{\mathrm{A}}=\frac{1}{2}, \mathrm{~A} / \mathrm{r}_{\mathrm{A}}=\frac{1}{3}$, whereas Figure 8.3 is plotted for a straight tube. Preliminary conclusions can be drawn from these results.

The data clearly indicate that in the lower Péclet numbers $(<10)$ the Sherwood number depends upon the packing depth. However, as the Péclet number increases this length dependence disappears. For Péclet numbers greater than 100 , there is no distinction between the Sherwood numbers in a bed of $\mathrm{aL}=10$ vs $\mathrm{aL}=100$. At this Péclet number, the Reynolds number was approximately $\frac{100}{2000}=0.05$.

Equation 1 could never reproduce this trend. It shows the strongest length dependence as the Péclet number increases due to the $\mathrm{Sh}_{\mathrm{B}}^{(3)}$ term. The combination of asymptotes must be reformulated. 
Figure 8.2 Combination of sinusoidal PCT asymptotic Sherwood numbers according to equation 1 with $\mathrm{r}_{\mathrm{A}}=\frac{1}{2}, \mathrm{~A} / \mathrm{r}_{\mathrm{A}}=\frac{1}{3}, \varepsilon=0.4$ and $\mathrm{n}=0.42$.

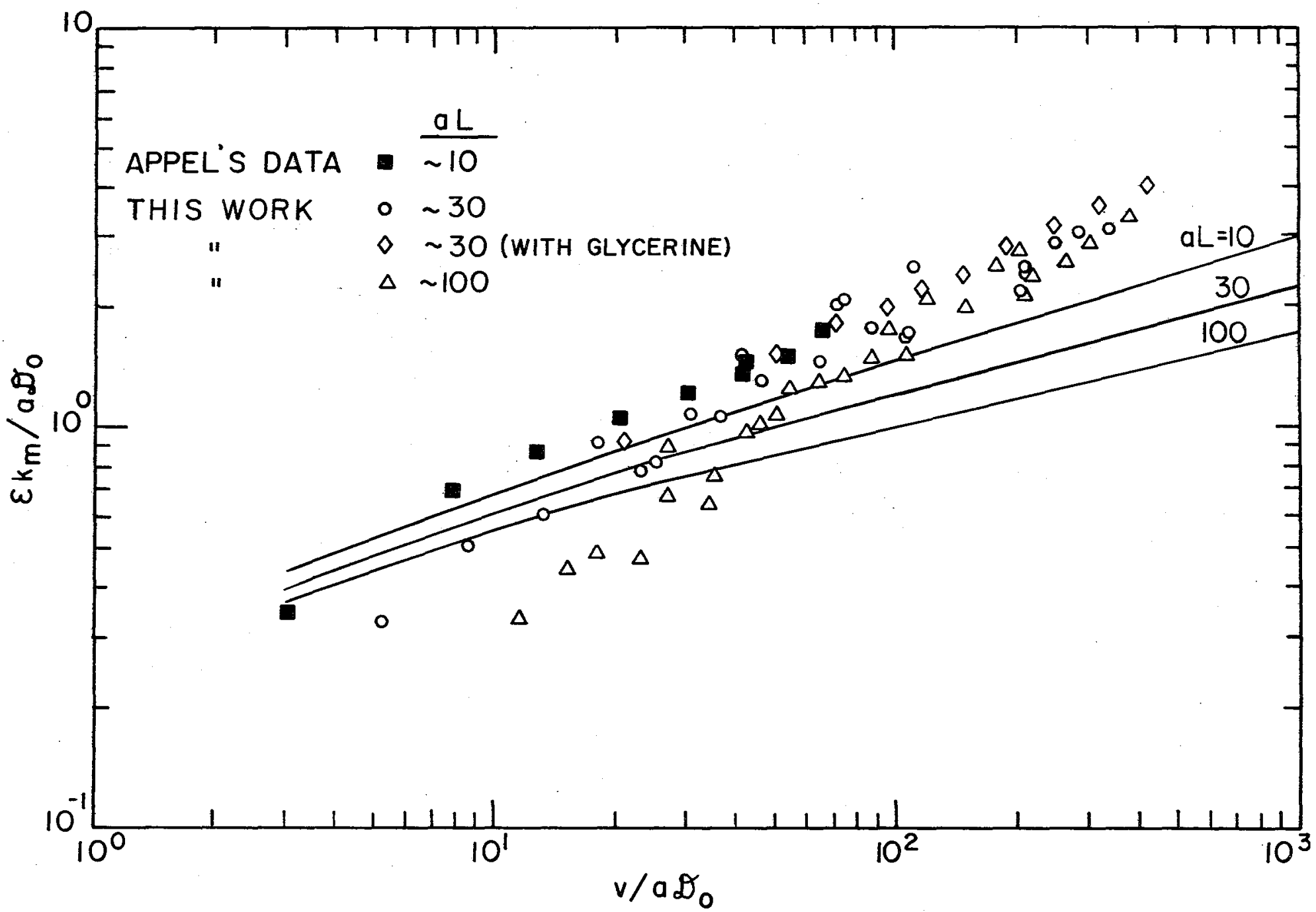


Figure 8.3 Combination of straight tube asymptotic Sherwood numbers according to equation 1 with $\varepsilon=0.4$ and $\mathrm{n}=0.50$.

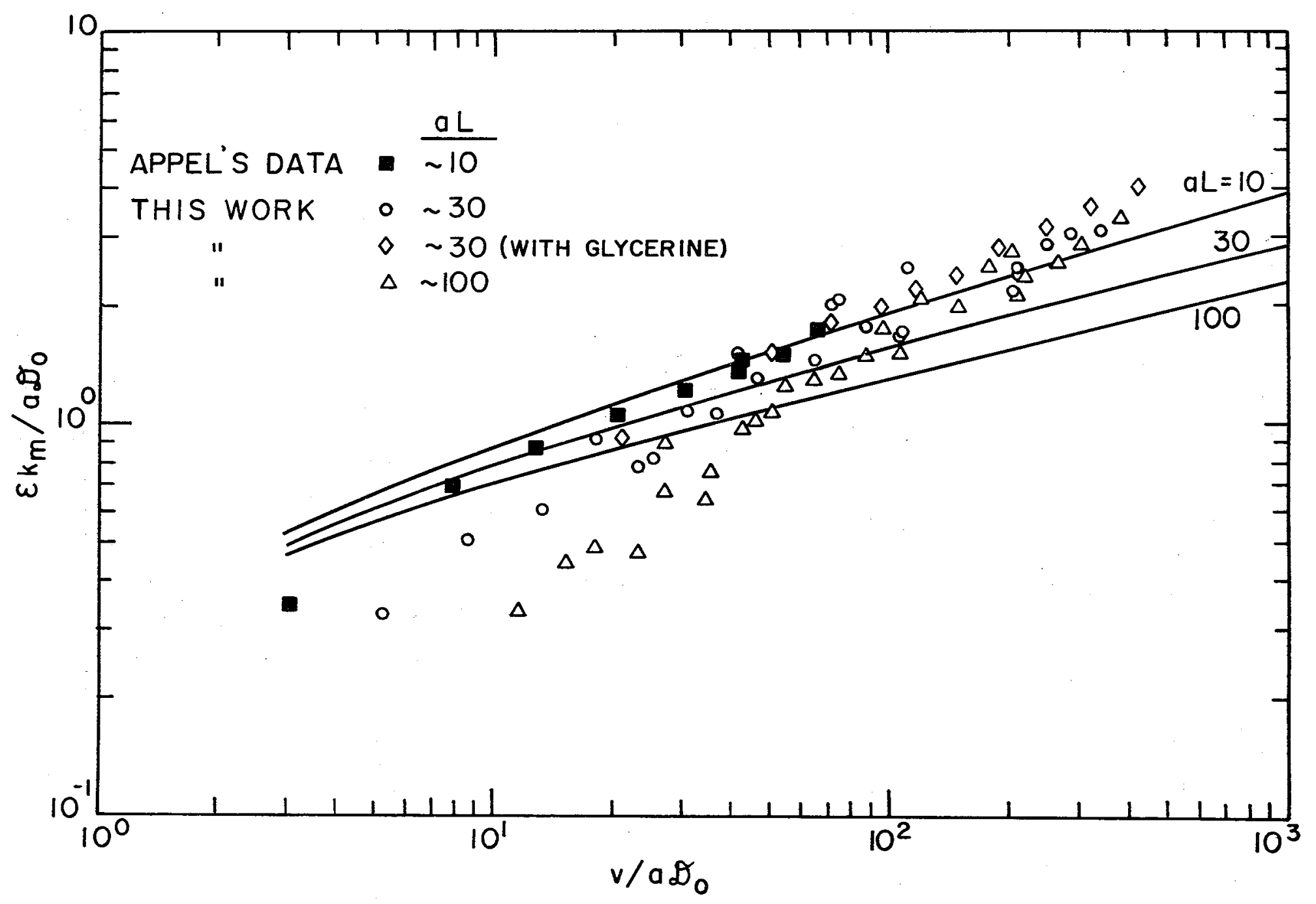

XBL $7811-6139$ 
In the high Péclet number region, the model assumed that the boundary layer formed along the particle surface retained its identity throughout the depth of the bed. It was anticipated that this would be true only in the creeping flow regime because any inertial caused mixing effects at higher Reynolds numbers would destroy the boundary layers. The data indicate, however, that the boundary layers do lose their identity. One might speculate as to the cause of this phenomenon at such low Reynolds numbers. Perhaps the lateral mixing of streams due to the random placement of the particles (which is not taken into account in the model) contributes to the destruction of the boundary layers.

No matter what the mixing mechanism, an empirical Reynolds number dependence may be incorporated into an analog of equation 1 . In this manner, the asymptotic Sherwood numbers are now combined as

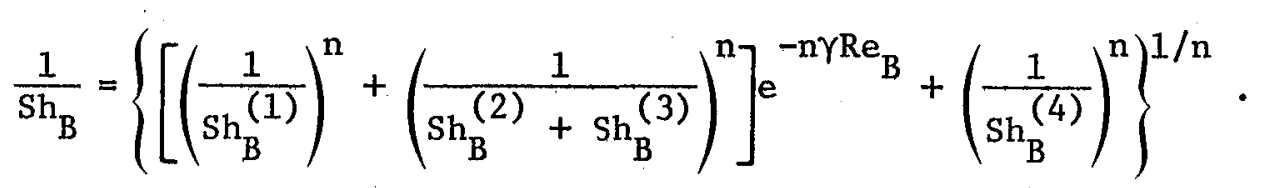

The exponential term involving the Reynolds number will cause the contribution of the length dependent term to become negligible compared to the non-length dependent term as the Reynolds number increases. There are now two parameters to fit to the data, $n$ and $\gamma$. The value of these parameters is again dependent upon the geometric parameters of the tube. 
Figure 8.4 and 8.5 are plots of equation 2 for a sinusoidal PCT of $\mathrm{r}_{\mathrm{A}}=\frac{1}{2}, \mathrm{~A} / \mathrm{r}_{\mathrm{A}}=\frac{1}{3}$ and the straight tube, respectively. The parameter values are listed in the figure caption.

The high Péclet number data are fitted excellently by the straight tube model but less so by the sinusoldal PCT. However, neither model can satisfactorily fit the lower Péclet number data. Both models overestimate the Sherwood number and, as with equation 1 , a strong enough length dependence is not predicted. These lower Péclet number data point to a weakness in the model which will be discussed shortly.

The sinusoidal PCT cannot reproduce the higher Péclet number data obtained in Chapter 7 as well as the straight tube model. For all values of the PCT geometrical parameters reported in the earlier chapters, the PCT calculations consistently underestimate the masstransfer coefficient. The level of success obtained in fitting the high Péclet number mass-transfer coefficients is further emphasized when the data of other investigators are considered.

Figure 8.6 is a plot of mass-transport limited Sherwood numbers compiled from 9 publications along with the data collected in the present study. All of the data are for Reynolds numbers. ( $v / a v)$ less than one. Drawn on this figure are the curves shown in Figures 8.4 and 8.5 for aL $=10$. It should be emphasized that these curves were fit to the results obtained in this and Appel's work. The straight tube calculations fit this collected data set at higher Péclet 
Figure 8.4 Combination of sinusoidal PCT asymptotic Sherwood numbers according to equation 2 with $\mathrm{r}_{\mathrm{A}}=1 / 2, \mathrm{~A} / \mathrm{r}_{\mathrm{A}}=1 / 3, \varepsilon=0.4, \mathrm{Sc}=2000, \gamma=1380$ and $\mathrm{n}=0.615$.

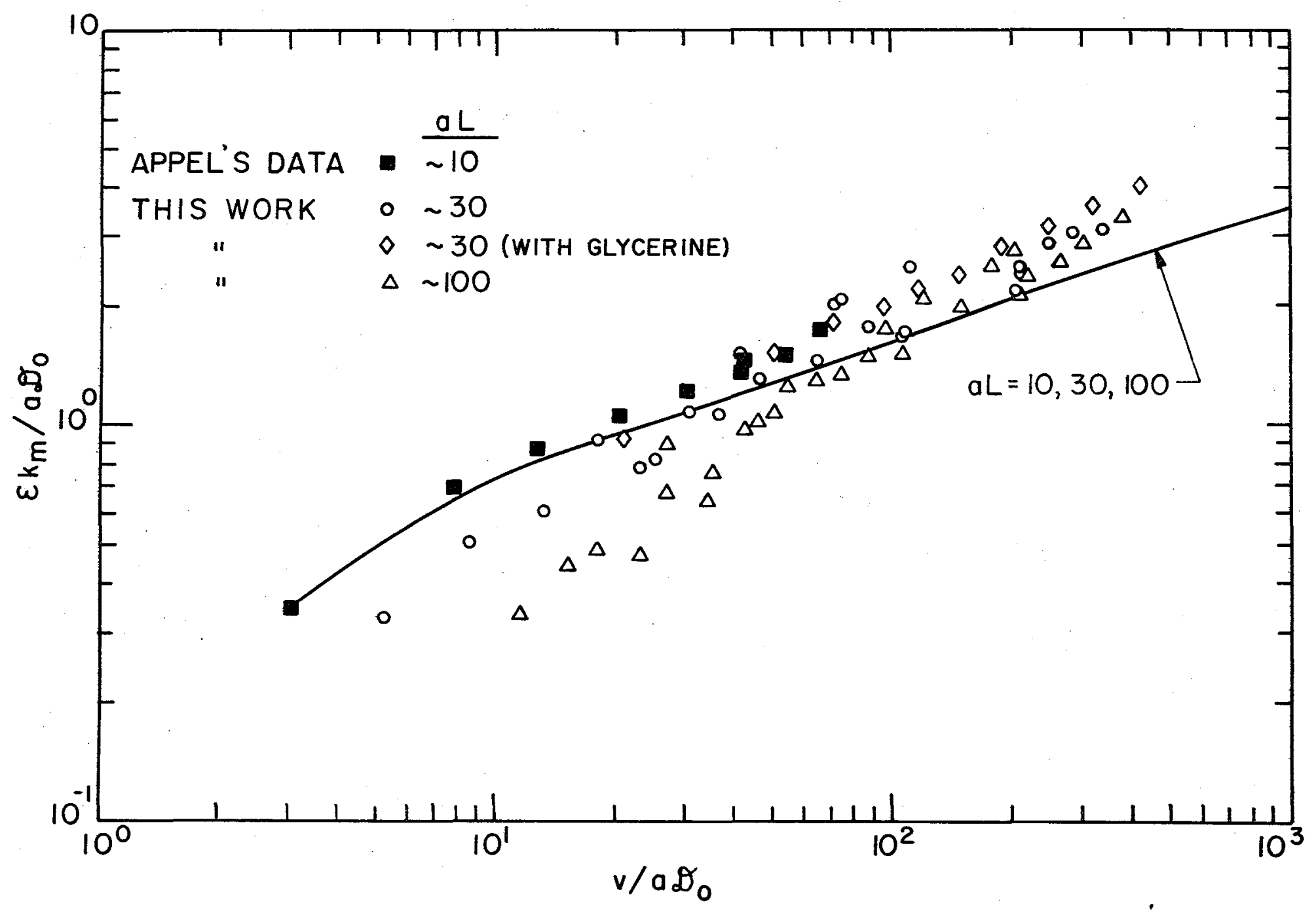


Figure 8.5 Combination of straight tube asymptotic Sherwood numbers according to equation 2 with $\varepsilon=0.4, \mathrm{Sc}=2000, \gamma=14.7$, and $\mathrm{n}=1.92$.

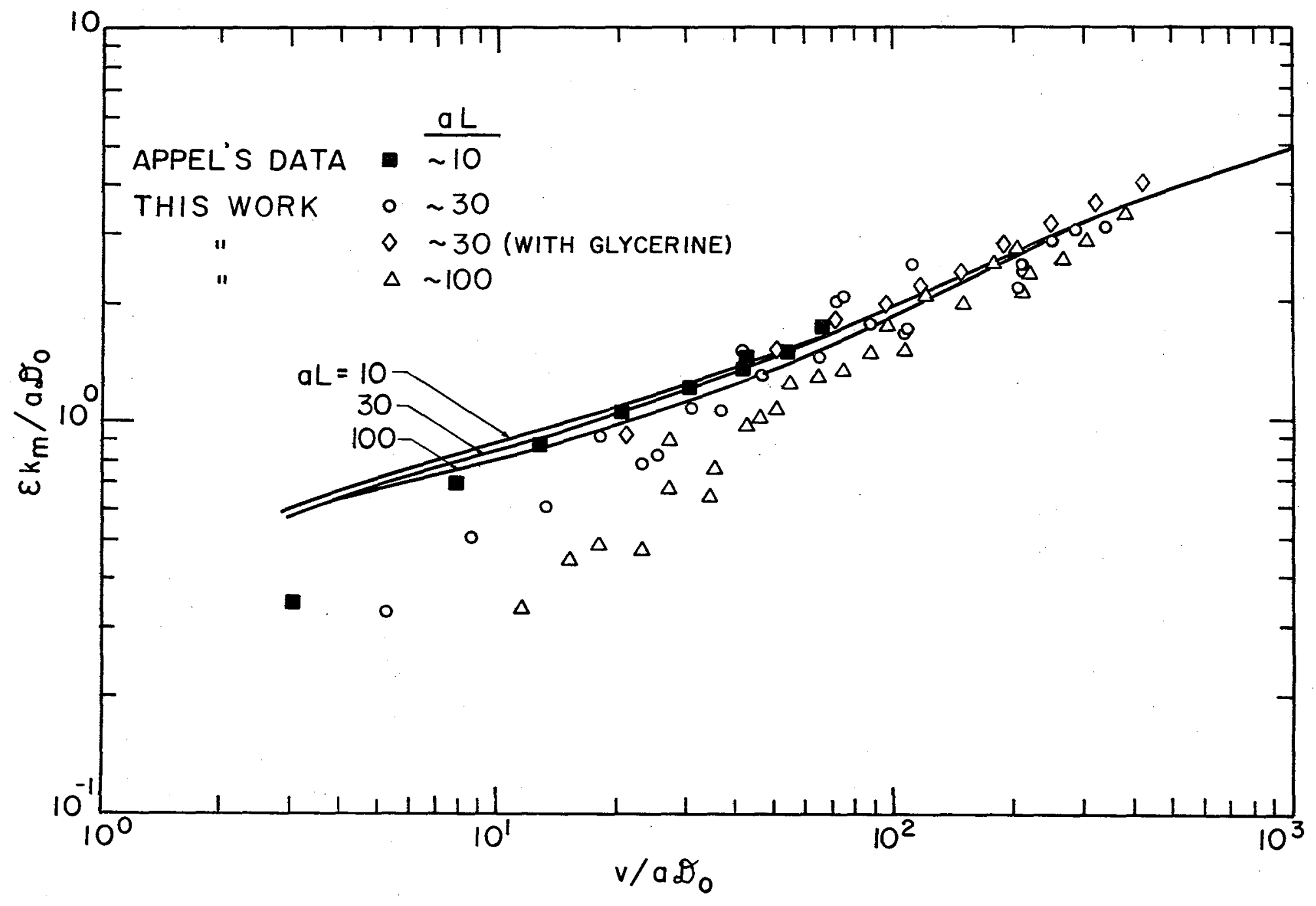




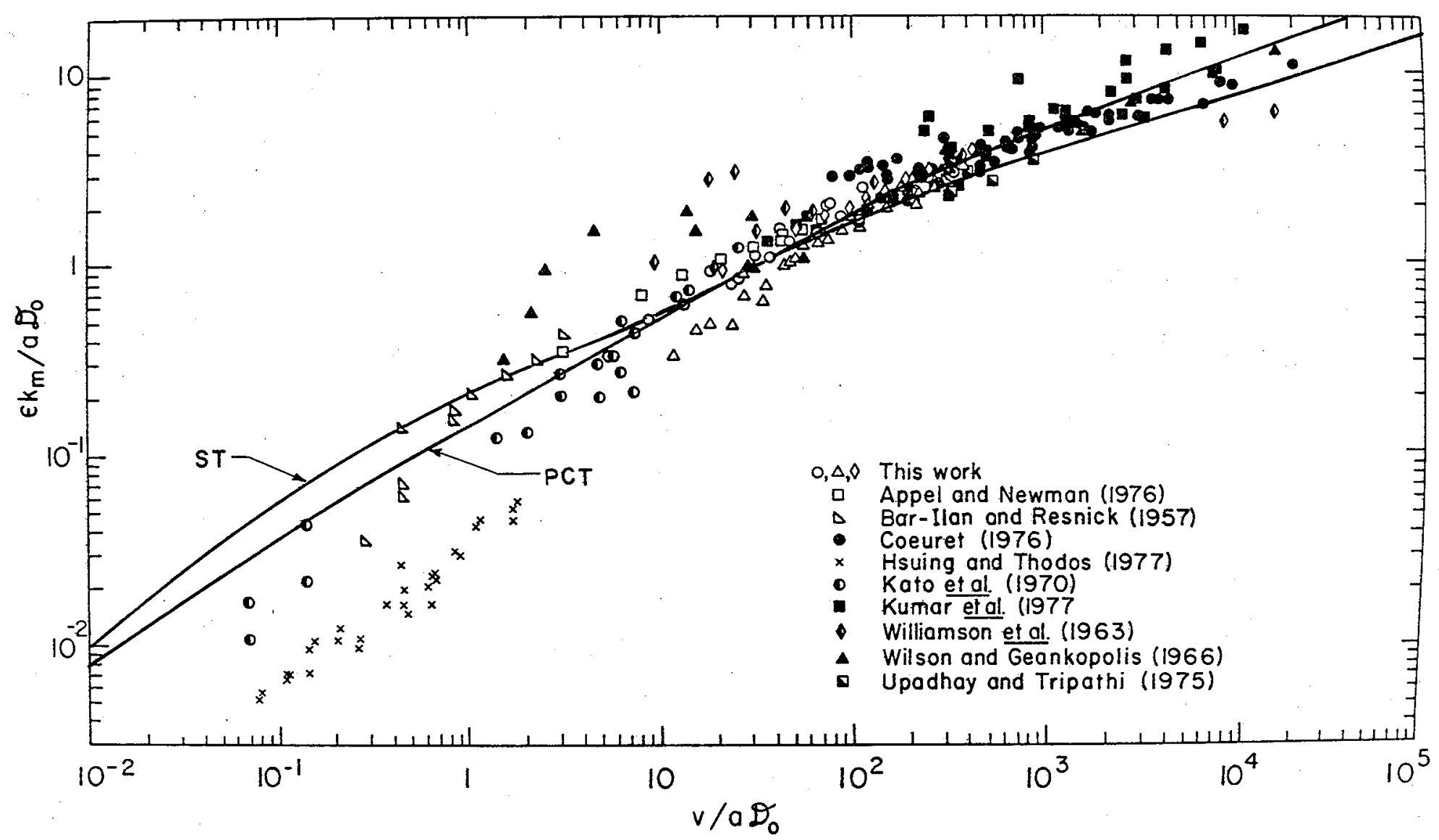

XBL $7811-13112$

Figure 8.6 The sinusoidal PCT and straight tube model Sherwood numbers for a packed bed according to equation 2. The parameter values are identical to those listed in the previous two figure captions. 
numbers much more satisfactorily than the PCT calculations. On this basis, one can conclude that the PCT model is not successful in fitting packed bed mass transfer data. The higher level of complexity required in calculating the PCT velocity profiles and asymptotic Sherwood numbers is not necessary. The PCT model was thought a priori to have been a better model for the bed because it could, in a sense, reproduce the constrictions and expansions that the actual fluid path must follow in a bed. This assumption has been proved wrong by this work.

In the remainder of this work, the straight tube model calculations are exploited, and the sinusoidal PCT results are abandoned as a model for packed beds.

The Effect of Flow Maldistribution in a Packed Bed on the Mass Transfer Coefficient

The low Péclet number mass-transfer coefficients obtained in this work show a stronger length dependence than is predicted by any model calculations. The full solution to the convective diffusion equation will give a length dependent coefficient for all values of the Péclet number, but this dependence is weakest in the lower Pêclet number regions. Table 8.1 supports this statement. This table shows the numerically calculated $k_{m}$ coefficients of $S \phi r e n s e n$ and Stewart for a simple cubic packed bed of uniform size spheres. The fifth column gives the ratio of the $k_{m}$ for a bed with aL $=9.9$ to that for a deep bed $(\mathrm{aL}=\infty)$. For a Péclet number of 9.6 , this ratio is 1.29 , whereas the data collected in this work give a value for this ratio of 2.8 when the aL ratio is $10: 100$. 
Table 8.1: Sфrensen and Stewart's calculated results for the masstransfer coefficient in a simple cubic packed bed of uniform sized spheres.

\begin{tabular}{|c|c|c|c|c|c|}
\hline & (1) & (2) & (3) & (4) & (5) \\
\hline 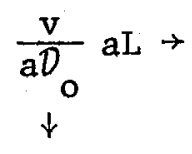 & 4.9 & 9.9 & 15 & $\infty$ & $(2) /(4)$ \\
\hline 9.55 & 0.916 & 0.769 & 0.714 & 0.595 & 1.29 \\
\hline 31.8 & 1.37 & 1.09 & 0.954 & 0.598 & 1.82 \\
\hline 95.5 & 2.11 & 1.59 & 1.38 & 0.603 & 2.64 \\
\hline 318 & 3.18 & 2.55 & 2.18 & 0.614 & 4.15 \\
\hline
\end{tabular}


The larger than anticipated length effect can be explained by a nonuniform flow distribution in a packed bed. The effect of channeling on processes taking place in a bed has long been recognized in the literature. The fluid may find preferential paths of least resistance through the bed. These lower resistance paths may be near the wall where the local porosity is higher than the bulk average, but these paths are not necessarily confined to the wall. Dullien (14) has pointed out that in randomly packed beds there is a finite probability for flow connections of a larger than average size to form a network and transverse the entire length of the bed.

Schlünder (106) has discussed the effect of flow maldistribution in an array of tubes. His array consisted of one large diameter tube embedded in a matrix of smaller sized tubes. Martin (107) has expanded upon this idea and applied it to a packed bed. In this work he considered the bed to consist of two regions, an annular outer region where the porosity is high and a central core region with the bulk porosity. Both workers have demonstrated that the overa11 mass-transfer coefficient one would calculate by appropriately summing the contributions of each flow segment is lower than that of a composite system where the nonuniformities are neglected by an averaging process. However, both workers have used the incorrect limiting form of the mass-transfer coefficient in their segmented flow channels. Schlünder recommended for each tube size the empirical combination of the Graetz and Lévêque solution, which is much like the combination suggested earlier in this chapter. This combination cannot reproduce the correct 1inear dependence 
of $k_{\mathrm{m}}$ on the Pêclet number in the low Pêclet number limit. Martin has made a similar conceptual error by applying the Ranz equation in each segment, which predicts that the Sherwood number (defined on the particle diameter basis) reduces to 2 as the Péclet number approaches zero.

In the following analysis this idea of flow maldistribution is expanded upon, and in the process the correct limiting form for the Sherwood number is used.

The bed is now considered to be an array of two different size radii straight tubes. In this manner, the channeling flow is not conceptually limited to the confining wall region. Each of these tubes has its associated radius $r_{1}$ and $r_{2}$, and its associated pore space $\varepsilon_{1}$ and $\varepsilon_{2}$ such that the total bed porosity is the sum of $\varepsilon_{1}$ and $\varepsilon_{2}$. Two dimensionless geometry parameters are generated by this model, the ratio of tube radii $r_{2} / x_{1}=\delta$, and the porosity ratio $\varepsilon_{2} / \varepsilon_{1}$.

Since the pressure gradient is assumed to be identical in all tubes, the ratio of the flowrates can be calculated by using the Hagen-Poiseuille solution

$$
\mathrm{q}_{2} / \mathrm{q}_{1}=\delta^{4}
$$

The lower case $q_{i}$ is used to designate the flowrate in a single tube of radius $r_{i}$. The upper case $Q_{i}$ will be used to designate the flowrate in the entire collection of tubes of radius $r_{i}$. 
The flowrate through the bed and the concentration at the exit of the bed may be written as

$$
\begin{aligned}
& Q=Q_{1}+Q_{2} \\
& Q_{L}=Q_{1} c_{L_{1}}+Q_{2} c_{L_{2}} .
\end{aligned}
$$

Define $\gamma$ as the ratio of $Q_{2}$ to $Q_{1}$; equation 5 is then written as

$$
\frac{c_{L}}{c_{F}}=\frac{1}{1+\gamma} \frac{{ }_{L_{1}}}{c_{F}}+\frac{\gamma}{1+\gamma} \frac{{ }_{L_{2}}}{c_{F}} .
$$

The overall Sherwood number for the bed is defined as usual

$$
\frac{{ }^{C} L}{c_{F}}=\exp \left[-\frac{S h_{B}}{P e_{B}} \frac{a L}{\varepsilon}\right] .
$$

Equation 6 can now be rearranged to calculate the overall bed Sherwood number in terms of the Sherwood number in each individual tube matrix.

$$
\begin{aligned}
\frac{\varepsilon k_{m}}{a D_{0}}= & \frac{\varepsilon}{\varepsilon_{1}} \frac{a_{1} L}{\mathrm{aL}^{L}} \frac{\mathrm{Pe}_{\mathrm{B}}}{\mathrm{Pe}_{1}} \mathrm{Sh}_{1}-\frac{\varepsilon}{\mathrm{aL}} \mathrm{Pe}_{\mathrm{B}} \ln \left\{\frac{1}{1+\gamma}+\frac{\gamma}{\gamma+1}\right. \\
& \left.\exp \left[\frac{\mathrm{a}_{1} \mathrm{~L}}{\varepsilon} \frac{\mathrm{Sh}}{\mathrm{Pe}_{1}}\left(1-\frac{\varepsilon_{1}}{\varepsilon_{2}} \frac{\mathrm{a}_{2} \mathrm{~L}}{\mathrm{a}_{1} \mathrm{~L}} \frac{\mathrm{Pe}_{1}}{\mathrm{Pe}_{2}} \frac{\mathrm{Sh}_{2}}{\mathrm{Sh}_{1}}\right)\right]\right\} .
\end{aligned}
$$

The Sherwood numbers $\mathrm{Sh}_{i}$ have been defined as

$$
\mathrm{Sh}_{i}=\frac{\varepsilon_{i} \mathrm{k}_{\mathrm{m}_{i}}}{a_{i} D_{0}}
$$


and the Péclet numbers $\mathrm{Pe}_{i}$ are defined as

$$
P e_{i}=\frac{v_{i}}{a_{1} D_{0}}
$$

with $v_{i}$ being the superficial velocity in the bed of tube size $r_{i}$ only. With $v_{i}$ so defined, the superficial velocity for the entire bed follows as the sum of $v_{1}$ and $v_{2}$.

Equation 8 can be placed Into a more useful form by eliminating the tube variables on the right side in terms of the macroscopic parameters for the entire bed. It is straightforward to derive the following relationships

$$
\begin{aligned}
& \mathrm{Pe}_{1}=\frac{1+\varepsilon_{2} / \varepsilon_{1} \delta}{1+\varepsilon_{2} \delta^{2} / \varepsilon_{1}} \mathrm{Pe}_{\mathrm{B}} \\
& \mathrm{Pe}_{2}=\frac{1+\varepsilon_{1} \delta / \varepsilon_{2}}{1+\varepsilon_{1} / \varepsilon_{2} \delta^{2}} \mathrm{Pe}_{B} \\
& \mathrm{a}_{1} \mathrm{~L}=\frac{\mathrm{aL}}{1+\varepsilon_{2} / \varepsilon_{1} \delta} \\
& \mathrm{a}_{2} \mathrm{~L}=\frac{\mathrm{aL}}{1+\varepsilon_{1} \delta / \varepsilon_{2}} .
\end{aligned}
$$

These relationships can be used in equation 8 to write 


$$
\begin{gathered}
\mathrm{Sh}_{\mathrm{B}}=\mathrm{hSh}_{1}-\frac{\varepsilon}{\mathrm{aL}} \mathrm{Pe}_{\mathrm{B}} \ln \left\{\frac{1}{1+\varepsilon_{2} \delta^{2} / \varepsilon_{1}}+\frac{\varepsilon_{2} \delta^{2} / \varepsilon_{1}}{1+\varepsilon_{2} \delta^{2} / \varepsilon_{1}}\right. \\
\left.\quad \exp \left[\mathrm{h} \frac{\mathrm{aL}}{\varepsilon} \frac{\mathrm{Sh}_{1}}{\mathrm{Pe}_{\mathrm{B}}}\left(1-\frac{\mathrm{Sh}_{2}}{\delta^{4} \mathrm{Sh}_{1}}\right)\right]\right\}
\end{gathered}
$$

where $h$ is defined as

$$
h=h\left(\delta, \varepsilon_{2} / \varepsilon_{1}\right)=\frac{\left(1+\varepsilon_{2} / \varepsilon_{1}\right)\left(1+\varepsilon_{2} \delta^{2} / \varepsilon_{1}\right)}{\left(1+\varepsilon_{2} / \varepsilon_{1} \delta\right)^{2}}
$$

By modeling the bed as an array of dual sized tubes, equation 15 can be used to calculate the overall conversion in the bed taking into account the flow distribution and the availability of reactive surface area in the network. The mass-transfer coefficients for each size tube matrix must be known in order to make use of this result. Our attention is now turned to this matter.

The data gathered in Chapter 7 indicate that there is no significant length dependence for $\mathrm{k}_{\mathrm{m}}$ in the higher Péclet number region. Earlier in this chapter, it was found necessary to include an exponential damping term involving the Reynolds number to reproduce this behavior when length dependent coefficients were empirically combined. These facts suggest that the Sherwood numbers for each tube size matrix be empirically combined as

$$
\frac{1}{S h_{i}}=\left\{\left[\frac{1}{\operatorname{Sh}_{i}^{(1)}}\right]^{n}+\left[\frac{1}{\operatorname{Sh}_{i}^{(4)}}\right]^{n}\right\}^{1 / n} .
$$


These Sherwood numbers for the straight tube model are

$$
\begin{aligned}
\operatorname{Sh}_{i}^{(1)} & =1.20 \mathrm{Pe}_{i} \\
\operatorname{Sh}_{i}^{(4)} & =0.896\left\{\frac{\varepsilon}{[6(1-\varepsilon)]^{2 / 3}} \frac{1+\varepsilon_{j} / \varepsilon_{i} \delta}{1+\varepsilon_{j} / \varepsilon_{i}}\right\}^{1 / 3} \mathrm{Pe}_{i}^{1 / 3} .
\end{aligned}
$$

There are now three parameters to fit to the data, $\delta, \varepsilon_{2} / \varepsilon_{1}$, and $\mathbf{n}$. Figure 8.7 is a plot of equation 15 compared to the data of Chapter 7. The parameter values were determined as before in a least squares sense and are listed in the figure caption. Figure 8.8 illustrates this equation with the fitted parameter set in comparison to the mass-transfer data of other workers.

This channeling model fits the data collected in this work for all Péclet numbers. The root-mean-square deviation is 10.8 percent. It also gives an excellent fit to the higher Péclet number data of the other workers. The low Péclet number data fit is not as good but is satisfactory.

The parameter set which fits the data collected in this work should not be expected to be the best set for other workers' beds. It is representative of the range, however, in which the values are expected to 1ie, and as seen in Figure 8.8 does give a satisfactory correlation.

Great care was taken in packing the bed used in this study to generate a reproducible packing and to minimize large void spaces. This is reflected in the porosity value for the larger tube size. 


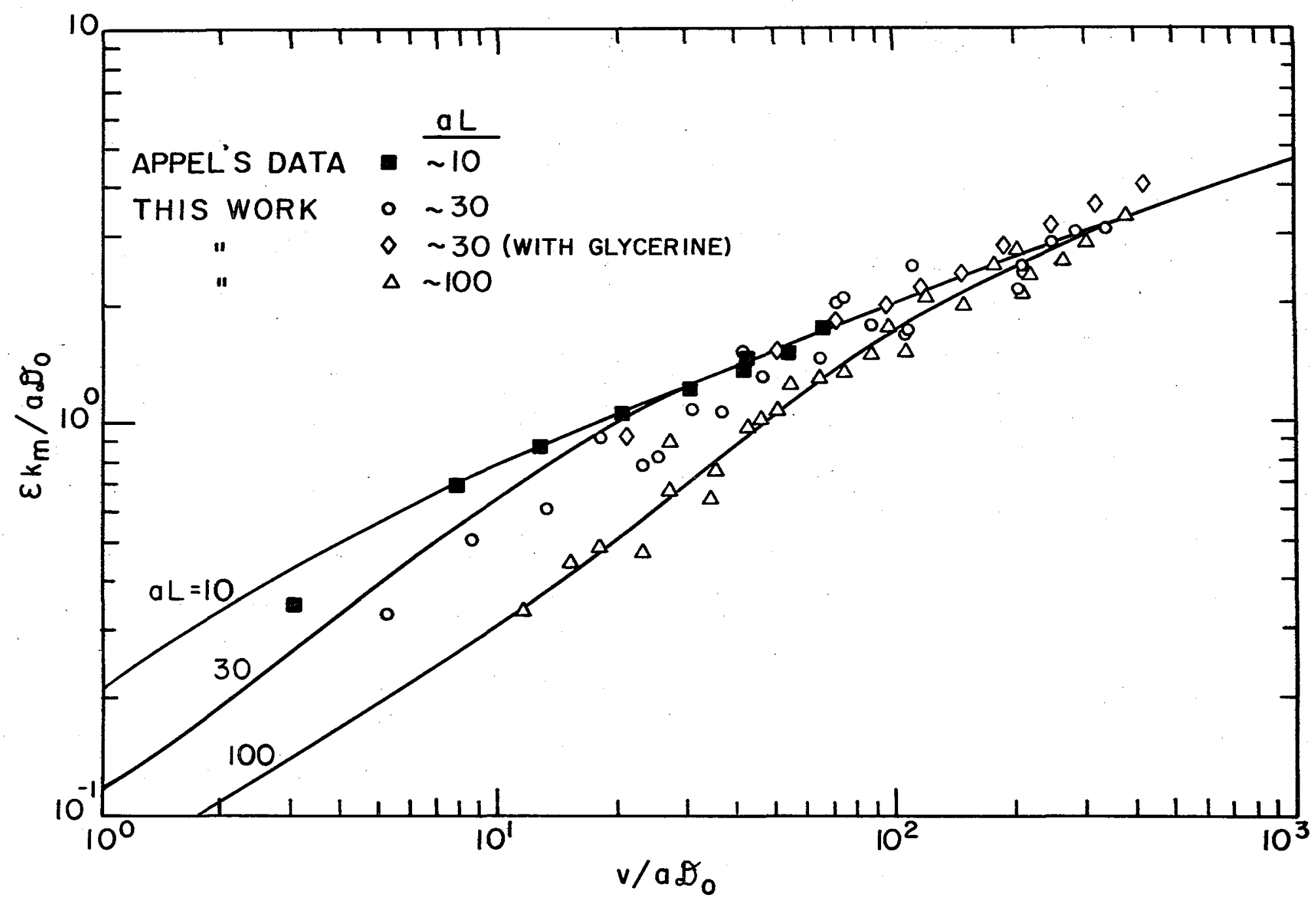

$X B L 78||-6 \mid 42$

Figure 8.7 Combination of straight tube asymptotic Sherwood numbers incorporating a channeling effect according to equation 15 with $\varepsilon=0.4, \mathrm{n}=0.642, \varepsilon_{2}=0.00584$ and $\delta=1.56$. 


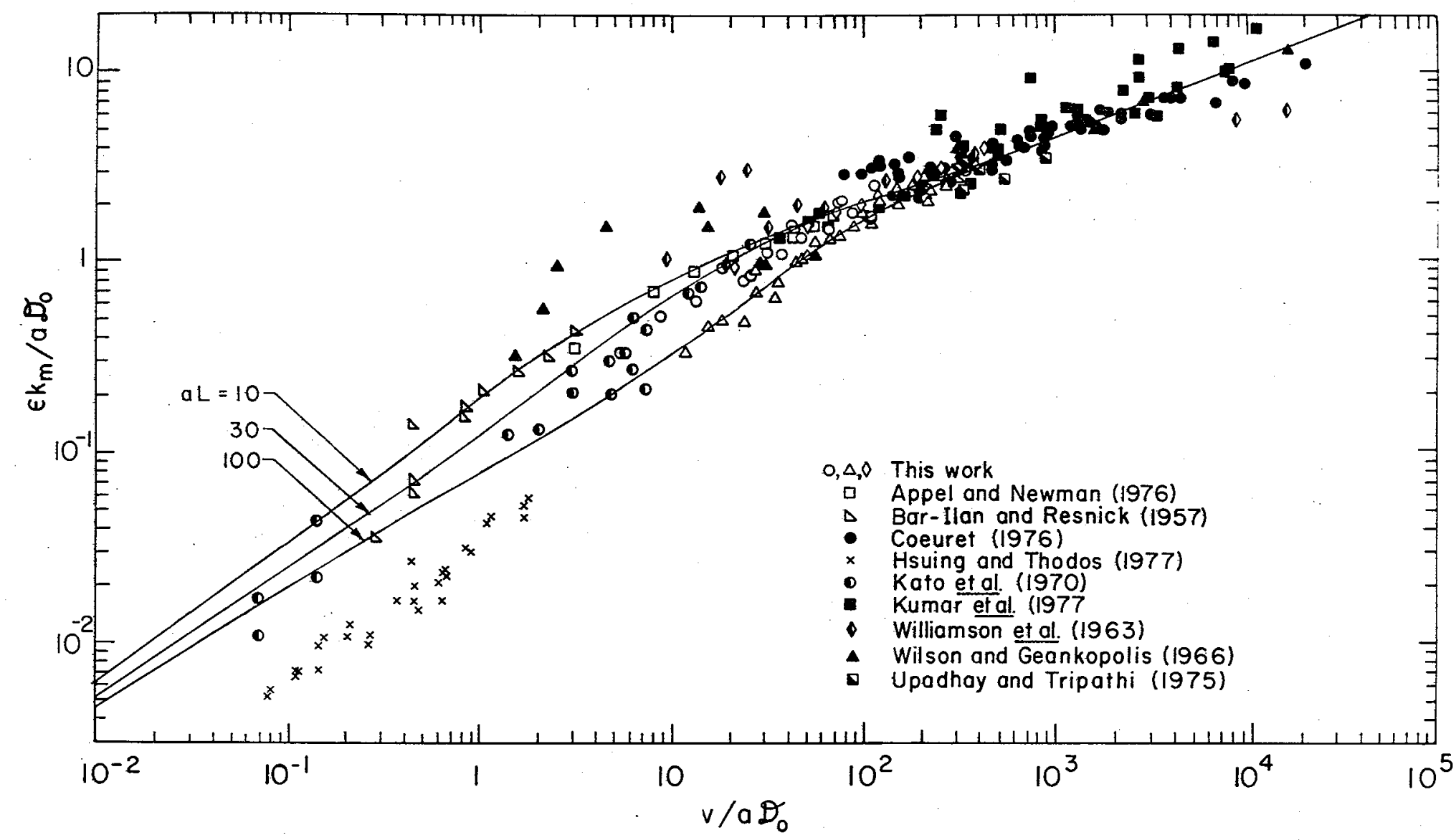

XBL 7811-13111

Figure 8.8 Comparison of straight tube channeling model with literature data. The parameter set is identical to that of figure 8.7. 
The larger void space occupies approximately $1.5 \%$ of the total void volume of the pack of this study. A non-tamped, randomly dumped bed would be expected to give a larger porosity value for the bigger tubes. This would also be true for beds of nonuntform size particles. Both of the above-mentioned beds would exhibit larger channeling flows and hence lower apparent mass-transfer coefficients. As a general rule, for a given Péclet number, the larger the fraction of fluid which channels through the bed, the smaller the apparent mass-transfer coefficient.

In terms of the two tube size model, the effect of channeling becomes insignificant at large Péclet numbers. Most of the reactant passes through the bed unreacted in this situation, therefore the width of the flow channel has very 1ittle effect. At low Péclet numbers, however, the channeling effect will always be apparent since the conversion at low Péclet numbers is controlled dominantly by the larger channels.

Equations 15, 17, 18, and 19 are a significant result of this thesis. The parameter values 1 isted in figure 8.7 may be used in this equation to correlate the transport controlled mass-transfer coefficient in packed beds. This correlation can then be used in the design of porous electrodes as outlined in Chapter 2. 


\section{NOMENCLATURE}

\section{Chapter 2}

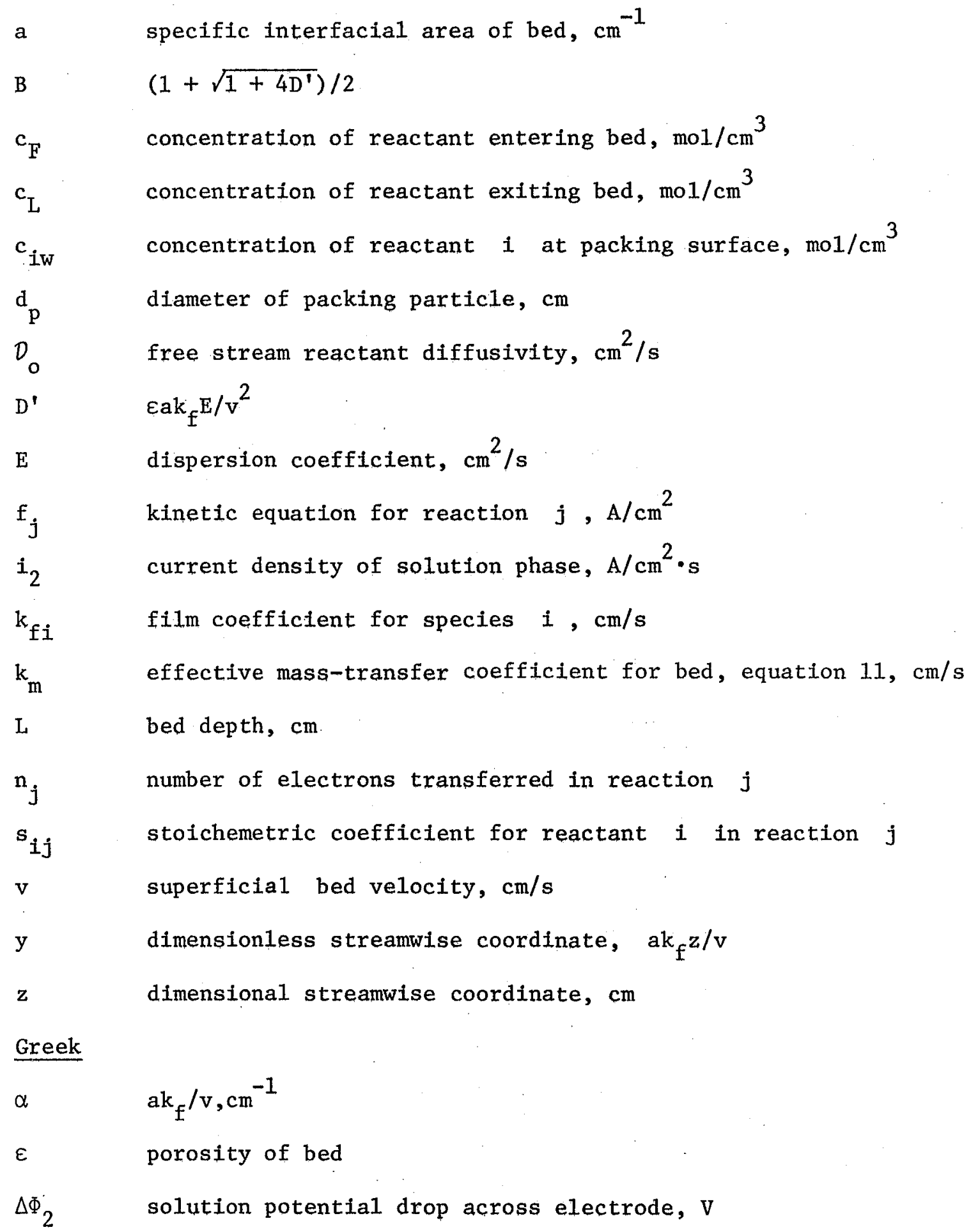




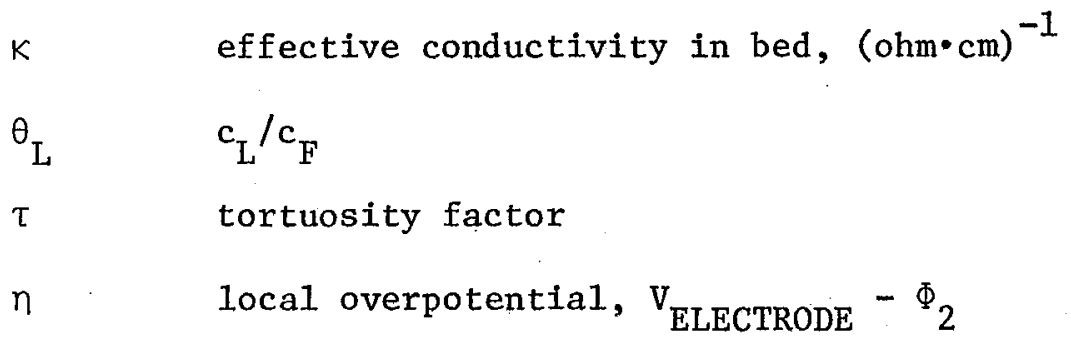

\section{Chapter 3}

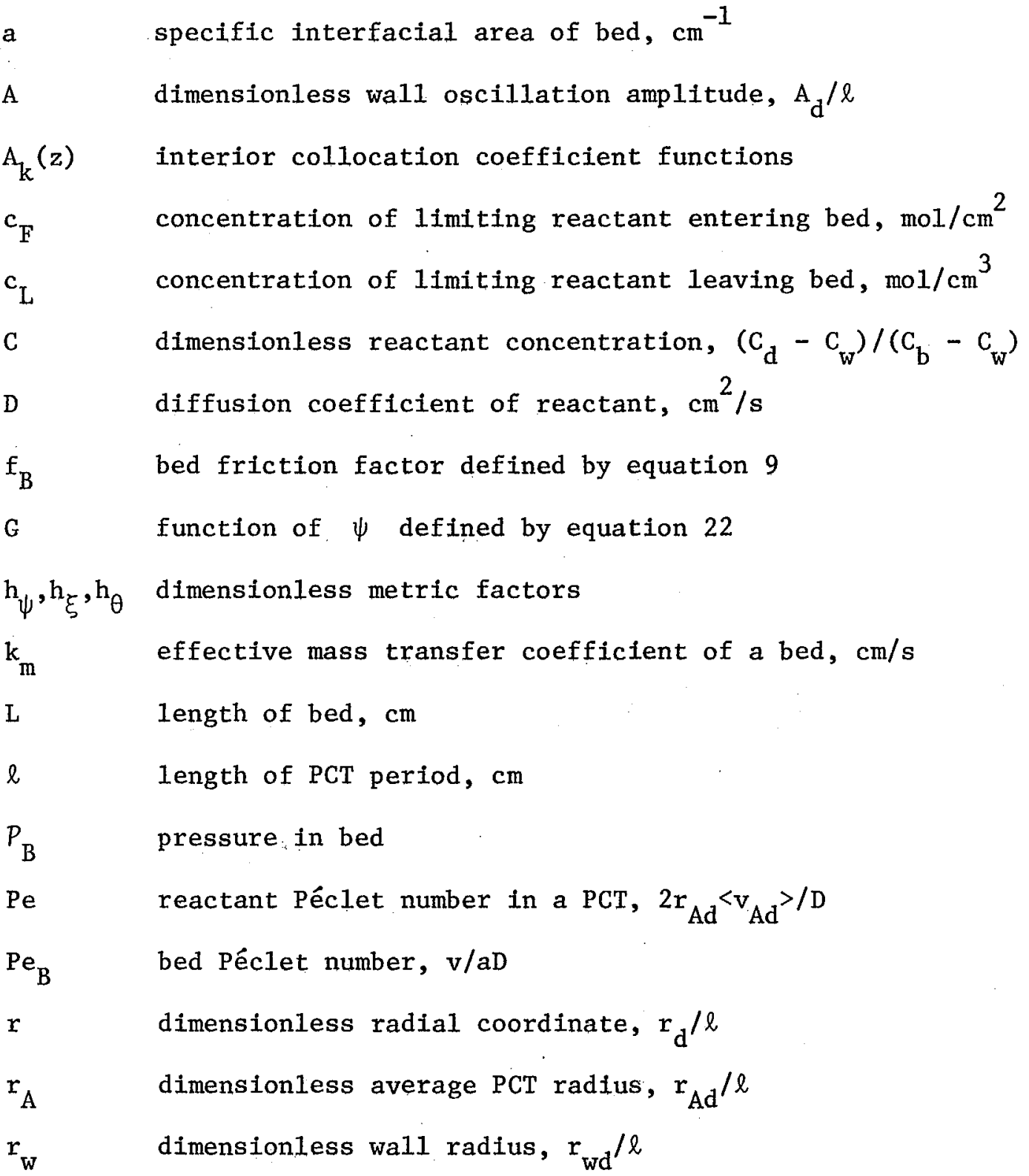




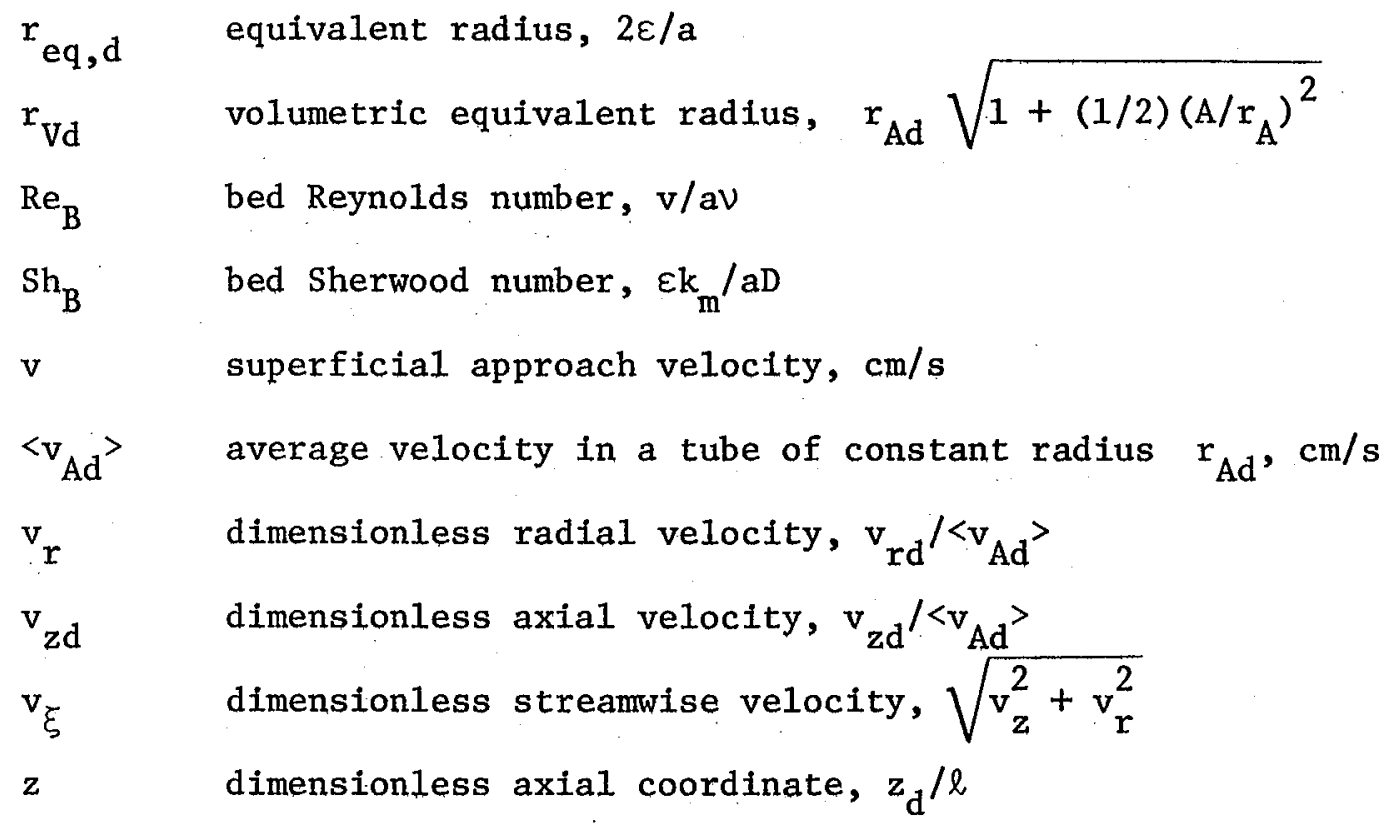

Greek

$\beta \quad$ constant defined by equation $10, \mathrm{~cm}^{-1}$

$\varepsilon \quad$ bed porosity

$v \quad$ kinematic viscosity, $\mathrm{cm}^{2} / \mathrm{s}$

$\xi \quad$ streamwise coordinate

$\rho \quad$ transformation coordinate of equation 28

$n \quad r / r_{w}(z)$

$\theta$ polar coordinate

$\lambda \quad$ eigenvalue of equation 21

$\psi \quad$ dimensionless normalized stream function, $-2 \psi_{\mathrm{d}} / \mathrm{r}_{\mathrm{Ad}}^{2}<\mathrm{v}_{\mathrm{Ad}}>$

$\left\{\phi_{k}\right\} \quad$ complete set of functions

Subscripts

b bulk

d dimensional quantity 


\section{Chapter 4}

a

specific interfacial area, $\mathrm{cm}^{-1}$

$c_{F} \quad$ far upstream reactant concentration, $\mathrm{mol} / \mathrm{cm}^{3}$

$c_{L} \quad$ far downstream reactant concentration, $\mathrm{mol} / \mathrm{cm}^{3}$

$D_{0} \quad$ molecular diffusion coefficient, $\mathrm{cm}^{2} / \mathrm{s}$

E dispersion coefficient, $\mathrm{cm}^{2} / \mathrm{s}$

$k_{f} \quad$ film mass-transfer coefficient, equation $3, \mathrm{~cm} / \mathrm{s}$

$k_{\mathrm{m}} \quad$ effective bed mass-transfer coefficient, equation $1, \mathrm{~cm} / \mathrm{s}$

L reaction section length, $\mathrm{cm}$

n normal coordinate, $\mathrm{cm}$

$\mathrm{Pe}_{\mathrm{B}} \quad$ bed Péclet number $\frac{\mathrm{v}}{\mathrm{a} \mathrm{D}_{\mathrm{O}}}$

$\mathrm{Sh}_{\mathrm{B}} \quad$ bed Sherwood number $\frac{\varepsilon \mathrm{k}_{\mathrm{m}}}{\mathrm{a} D_{\mathrm{O}}}$

v superficial velocity, $\mathrm{cm} / \mathrm{s}$

$x_{1}, x_{2}, x_{3}$ dimensionless bed coordinates, $a x_{d}$

z

streamwise dimensional coordinate

Greek

$\delta_{i j} \quad$ Kroenecker delta

$\varepsilon \quad$ porosity

$\tau \quad$ tortuosity

Subscripts

d

dimensional quantity

In

logarithmic mean 


\section{Chapter 5}

a

specific interfacial area, $\mathrm{cm}^{-1}$

$a_{k m}, b_{k m}$

Fourier expansion coefficients

$A_{k}(z)$

A

c

C

$D_{0}$

$\mathrm{J}$

$\mathrm{k}_{\mathrm{m}}$

$\ell$

L

$\mathrm{Pe}_{\mathrm{B}}$

$\mathrm{r}$

$\mathrm{r}_{\mathrm{A}}$

$r_{w}(z)$

$\mathrm{Sh}_{\mathrm{B}}$

$S t_{B}$

$\mathbf{v}$

Z

$\underline{\text { Greek }}$

$\alpha_{1}, \alpha_{2}$

$\gamma_{\mathrm{k}}$

$\varepsilon \quad$ bed porosity

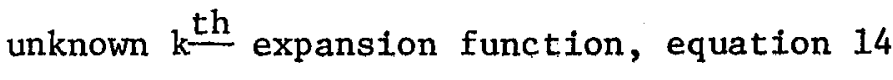

dimensionless wall oscillation amplitude, $A_{d} / \ell$

reactant concentration, $\mathrm{mol} / \mathrm{cm}^{3}$

transformation variable, equation 9

free stream diffusion coefficient, $\mathrm{cm}^{2} / \mathrm{s}$

Besse1 function of order zero

effective mass-transfer coefficient, $\mathrm{cm} / \mathrm{s}$

length of PCT period, $\mathrm{cm}$

length of reactive bed, $\mathrm{cm}$

bed Péclet number $\mathrm{v} / \mathrm{a} \mathrm{D}_{\mathrm{o}}$

dimensionless radial coordinate in a PCT, $\mathrm{r}_{\mathrm{d}} / \ell$

dimensionless average PCT radius, $\mathrm{r}_{\mathrm{Ad}} / \ell$

dimensionless wa11 radius, $\mathrm{r}_{w d} / \ell$

bed Sherwood number, $\frac{\varepsilon k_{m}}{a D_{0}}$

bed Stanton number $\mathrm{Sh}_{B} / \mathrm{Pe}_{B}, \frac{\varepsilon \mathrm{k}_{\mathrm{m}}}{\mathrm{v}}$

superficial bed velocity, $\mathrm{cm} / \mathrm{s}$

dimensionless axial coordinate in a PCT, $z_{d} / \ell$

$k \quad k \frac{\text { th }}{k}$ root of Bessel function $J_{0}$ 


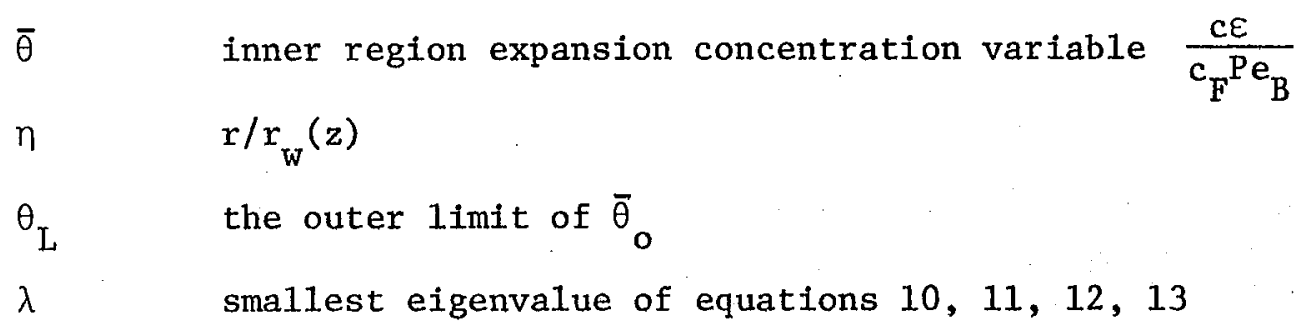




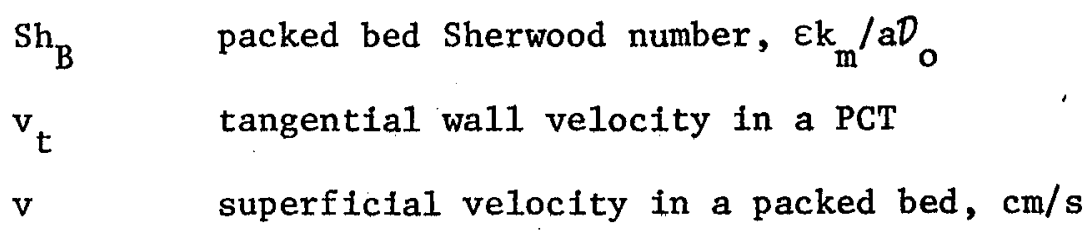

Subscript

d

indicates a dimensional quantity

\section{Greek}

$\begin{array}{ll}\varepsilon & \text { porosity } \\ \phi_{\mathrm{k}} & \text { radially dependent expansion function for stream function }\end{array}$

Chapter 7

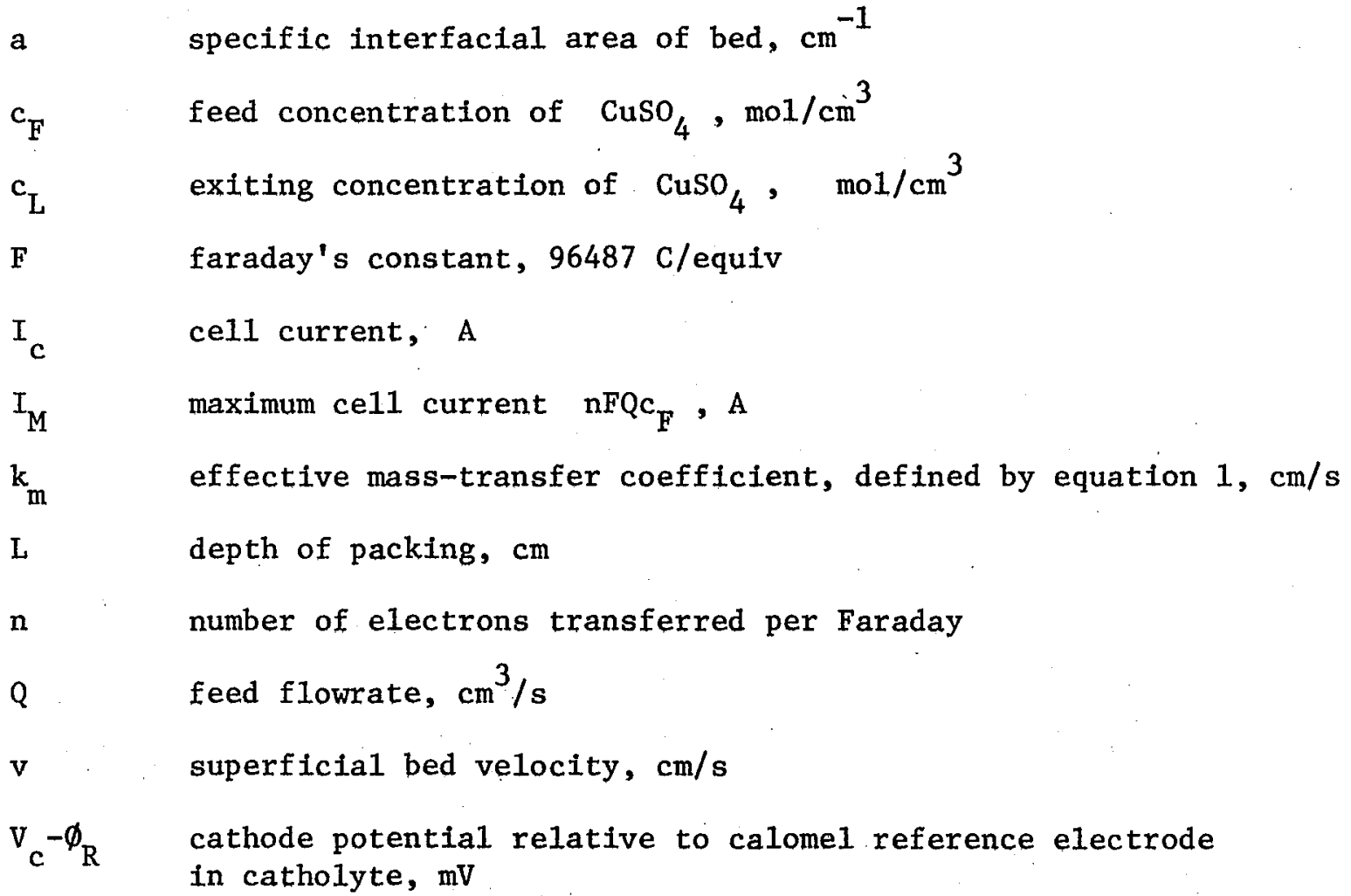


Greek

$\varepsilon_{i} \quad$ relative error in measurement of property $i$, defined by equation 3

$\varepsilon \quad$ bed porosity

$\theta_{\mathrm{L}} \quad \mathrm{c}_{\mathrm{L}} / \mathrm{c}_{\mathrm{F}}$

\section{Chapter 8}

$a_{i}$

a

$c_{F}$

$c_{L_{i}}$

$\mathrm{c}_{\mathrm{L}}$

Do

h

L

n

$\mathrm{Pe}_{\mathbf{i}}$

$\mathrm{Pe}_{\mathrm{B}}$

$q_{i}$

$Q_{i}$

$r_{i}$

$\mathrm{Sh}_{\mathrm{i}}$

$\mathrm{Sh}_{\mathrm{B}}$

$v_{i}$

$\mathrm{v}$ specific interfacial area of tube size $i$ matrix, $\mathrm{cm}^{-1}$ specific interfacial area of entire bed, $a_{1}+a_{2}, \mathrm{~cm}^{-1}$ reactant feed concentration, $\mathrm{mol} / \mathrm{cm}^{3}$ reactant concentration exiting from tube size $i$ matrix, $\mathrm{mol} / \mathrm{cm}^{3}$

reactant concentration exiting from bed, $\mathrm{mol} / \mathrm{cm}^{3}$

free stream reactant diffusivity, $\mathrm{cm}^{2} / \mathrm{s}$

defined by equation 16

bed length, cm

empirica1 constant

Péclet number for tube size $i$ matrix, $v_{i} / a_{i} D_{0}$

Péclet number for bed, $\mathrm{v} / \mathrm{a} \mathrm{D}_{\mathrm{o}}$

flowrate in tube size $i, \mathrm{~cm}^{3} / \mathrm{s}$

collective flowrate in tube size $i$ matrix, $\mathrm{cm}^{3} / \mathrm{s}$

radius of tube size $i, \mathrm{~cm}$

Sherwood number for tube size $i$ matirx, $\varepsilon_{i} k_{m_{i}} / a_{i} D_{0}$

Sherwood number for bed, $\varepsilon \mathrm{k}_{\mathrm{m}} / \mathrm{a} D_{\mathrm{o}}$

superficial velocity in tube size $i$ matrix, $\mathrm{cm} / \mathrm{s}$

superficial velocity in bed, $v_{1}+v_{2}, \mathrm{~cm} / \mathrm{s}$ 
Greek

$\begin{array}{ll}\gamma & Q_{2} / Q_{1} \\ \varepsilon_{i} & \text { porosity of tube size } 1 \text { matrix } \\ \varepsilon & \text { bed porosity, } \varepsilon_{1}+\varepsilon_{2} \\ \delta & \mathrm{r}_{2} / \mathrm{r}_{1}\end{array}$




\section{Appendix A}

$\mathrm{E}^{4}$ operator in $(n, z)$ coordinate system

$$
\begin{aligned}
& E^{4}=\frac{1}{r_{w}^{4}}\left[1+\eta^{2}\left(r_{w}^{\prime}\right)^{2}\right]^{2} \frac{\partial^{4}}{\partial \eta^{4}}-\frac{4 \eta}{r_{w}^{3}} r_{w}^{\prime}\left[1+\eta^{2}\left(r_{w}^{\prime}\right)^{2}\right] \frac{\partial^{4}}{\partial z \partial \eta^{3}} \\
& +\frac{1}{r_{w}^{2}}\left[2+6 \eta^{2}\left(r_{w}^{\prime}\right)^{2}\right] \frac{\partial^{4}}{\partial z^{2} \partial \eta^{2}}-\frac{4 \eta}{r_{w}} r_{w}^{\prime} \frac{\partial^{4}}{\partial z^{3} \partial \eta}+\frac{\partial^{4}}{\partial z^{4}} \\
& +\frac{1}{r_{w}^{4}}\left[-\frac{2}{\eta}+10 \eta\left(r_{w}^{\prime}\right)^{2}+12 \eta^{3}\left(r_{w}^{\prime}\right)^{4}-2 n r_{w} r_{w}^{\prime \prime}-6 \eta^{3} r_{w}\left(r_{w}^{\prime}\right)^{2} r_{w}^{\prime \prime}\right] \frac{\partial^{3}}{\partial \eta^{3}} \\
& +\frac{1}{r_{w}^{3}}\left[-4 r_{w}^{\prime}-24 \eta^{2}\left(r_{w}^{\prime}\right)^{3}+12 \eta^{2} r_{w} r_{w}^{\prime} r_{w}^{\prime \prime}\right] \frac{\partial^{3}}{\partial z \partial \eta^{2}} \\
& +\frac{1}{r_{w}^{2}}\left[12 \eta\left(r_{w}^{\prime}\right)^{2}-6 \eta r_{w} r_{w}^{\prime \prime}-\frac{2}{\eta}\right] \frac{\partial^{3}}{\partial z^{2} \partial \eta} \\
& +\frac{1}{r_{w}^{4}}\left[4\left(r_{w}^{\prime}\right)^{2}-2 r_{w} r_{w}^{\prime \prime}+\frac{3}{n^{2}}+36 n^{2}\left(r_{w}^{\prime}\right)^{4}+3 \eta^{2} r_{w}^{2}\left(r_{w}^{\prime \prime}\right)^{2}-36 \eta^{2} r_{w}\left(r_{w}^{\prime}\right)^{2} r_{w}^{\prime \prime}\right. \\
& \left.+4 \eta^{2} r_{w}^{2} r_{w}^{\prime} r_{w}^{\prime \prime}\right] \frac{\partial^{2}}{\partial \eta^{2}}+\frac{1}{r_{w}^{2}}\left[24 \eta r_{w}^{\prime} r_{w}^{\prime \prime}-\frac{24 \eta}{r_{w}}\left(r_{w}^{\prime}\right)^{3}\right. \\
& \left.+\frac{4}{\eta r_{w}} r_{w}^{\prime}-4 \eta r_{w} r_{w}^{\prime \prime \prime}\right] \frac{\partial^{2}}{\partial z \partial \eta}+\frac{1}{r_{w}^{4}}\left[-\frac{3}{\eta^{3}}-\frac{4}{\eta}\left(r_{w}^{\prime}\right)^{2}+24 \eta\left(r_{w}^{\prime}\right)^{4}\right. \\
& \left.-36 \eta r_{w}\left(r_{w}^{\prime}\right)^{2} r_{w}^{\prime \prime}+8 \eta r_{w}^{2} r_{w}^{\prime} r_{w}^{\prime \prime \prime}+6 \eta r_{w}^{2}\left(r_{w}^{\prime \prime}\right)^{2}+\frac{2}{\eta} r_{w} r_{w}^{\prime \prime}-\eta r_{w}^{3} r_{w}^{(i v)}\right] \frac{\partial}{\partial \eta}
\end{aligned}
$$




\section{Appendix B \\ Listing of Computer Programs}

This appendix contains the listing of the programs to perform the calculations presented in the main body of the thesis.

Each program is preceeded by an introduction which states the purpose of the routine and the necessary input parameters.

Many of these routines call upon identical subroutines. In order to save space, the subroutines are 1isted once where they first appear. 
Program INTER

This program solves for the unknown $A_{k}(z)$ functions of the stream function in a sinusoidal PCT as given in Chapter 3. The calculated $A_{k}(z)$ are printed as well as the velocity profiles at selected axial and radial positions. The program also calculates the Graetz-1ike eigenvalue in a sinusoidal PCT according to equations 3.29 and 3.30 .

The necessary input parameters are: NCS, the number of parameter sets to be processed; NCP, the number of $\eta$ coordinate collocation points; NJ, the number of axial mesh points used in the BAND subroutine; NSKIP, a parameter used to control the axial spacing of the printed velocity profiles; ZMIN and ZMAX, the origin and endpoint of the axial integration, either $(0,1 / 2)$ or $(0,1)$; RA and AMP, the average radius and amplitude of the sinusoidal PCT; NZ, the number of axial points used on the evaluation of $G(\psi)$ given by equation 3.22. 
PRUURAM INTER (JUNK, INPUT, CUTPUT)

EXTERNAL DELT

DIMENSION DELF(103),GG(201),V(201),VP(201),VTEN1201), FE(2)

DIMENSICN PIIOI,

$1 P 1(10), P 2(10), P 3(10), P 4(10), A 1(1,10), 42(1,10), 43(1,10), 44(1,1)$,

$2 C(20,103), G(20), A(20,20), B(20,20), 0(20,41), X(20,20), Y(20,20)$,

उAETA 10$)$

DOUB́L: PPECISION P,P1,P2,P3,P4, PT, PT I, FT2, PT 3, PT4,H,HL,H2,H3,H4,

1 HT, HT1, HT2,HT3,HT4, HOLD, A1, A2, A3, A4

DUUBLE PREC, ISICN FW, FWL, FW2, FW3, FWC, FWIC, FW2C, FW3C, TLA2, T1A3,

IT $2 A 3, T 3 A 3, T 1 A 4, T 2 A 4, T 3 A 4, T 4 A 4, T 1 A 5, T 2 A 5, T 3 A 5, T 4 A 5, A A 1, A A 2, A A 3, \triangle A 4$,

$2 A A 5$

COMMCN A, B, C, D, G, X,Y,N,NJ

C

COMMON /MAIN/ PSISTAR,NCP,J,P,P1,P2,P3,P4

C

SET UP ALL FUNCTICN STATEMENTS

$K W(L)=R A-A M P * \operatorname{COS}(2 \cdot * P I * Z)$

$K W I(\angle)=2 * \# I * A M P * \operatorname{SIN}(2 * \# I * Z)$

$R_{w 2}(Z)=4 * * A M P * \operatorname{CCS}(2 * * P I * Z) * P I * P I$

$K W 3(\angle)=-80 * A M P * \operatorname{SIN}(2 * * P I * Z) * P I * * 3$

$K w 4(Z)=-16$ 16*AMP*CกS $(2 * * P[Z) * P I * 4$

$F(E T A)=E T A * E T A *(1.0-2.0 * E T A * 2+E T A * * 4)$

F1(ETA) $=2$. ETA* $(1 .-40 *$ ETA*2* + 3.*TA*4)

F $2(E T A)=200-6 . * E T A * E T A *(40-50 *[T A * 2)$

$F 3(E T A)=-24$ *ETA* $(2.0-5 . * F T A * 2)$

$F 4(E T A)=24 . *(15 . * T A * 2-2.1$

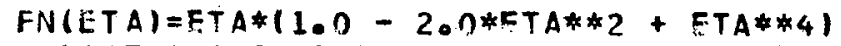

FNI $(E T A)=2.0 *(1.0-(4 \cdot-3 . *$ ETA*2)*FTA* 2$)$

$F W(Z)=P W 1(Z) / R W(Z)$

$F W I(Z)=R W 2(Z) / R W(Z)-F W(Z) * 2$

$F W 2(\angle)=R W 3(Z) / R W(Z)-3 * F W(Z) * R W 2(2) / R W(Z)+2 * * F(Z)$

$F W 3(2)=(R W 4(Z)-4 * F W(Z) * R W 3(Z)-3$ * *FW $1(2) * R W 2(2)+3$ * *RW2 $(Z) * F W(2) * 2$

$1+60 * R W(Z) * F W L(Z) * F W(Z) * 21 / R W(Z)$

HT (ETA,PT) $=F(E T A) * P T$

HTI(ETA,PT, PT 1$)=F 1(E T A) * P T+F(E T A) * P T 1$

HT2 $(E T A, P T, P T 1, P T 2)=F 2(E T A) * P T+20 * F 1(F T A) * P T 1+F(E T A) * P T 2$

HT 3 (ETA,PT,PT1,PT2,PT3)=F3(ETA)*PT+3.*F2(FTA)*PT1+3.*F1(ETA)*PT2+

1 F( ETAI *PT 3

HT4(ETA,PT,PT1,PT2,PT3,PT4)=F4(ETA)*PT +4.*F3(FTA)*PT1+60*F2(FT

1A)*PT $2+4$ * *F1 1 ETA)*PT3 + F(FTA)*PT 4

$P I=3 \cdot 141592654$

READ IN DATA

KEAD NUMBER OF COLLC SFT PEINTS TO BE PROCESSED

READ 5, NCS

5 FURMATII5)

Dr 999 III $=1$. NCS 


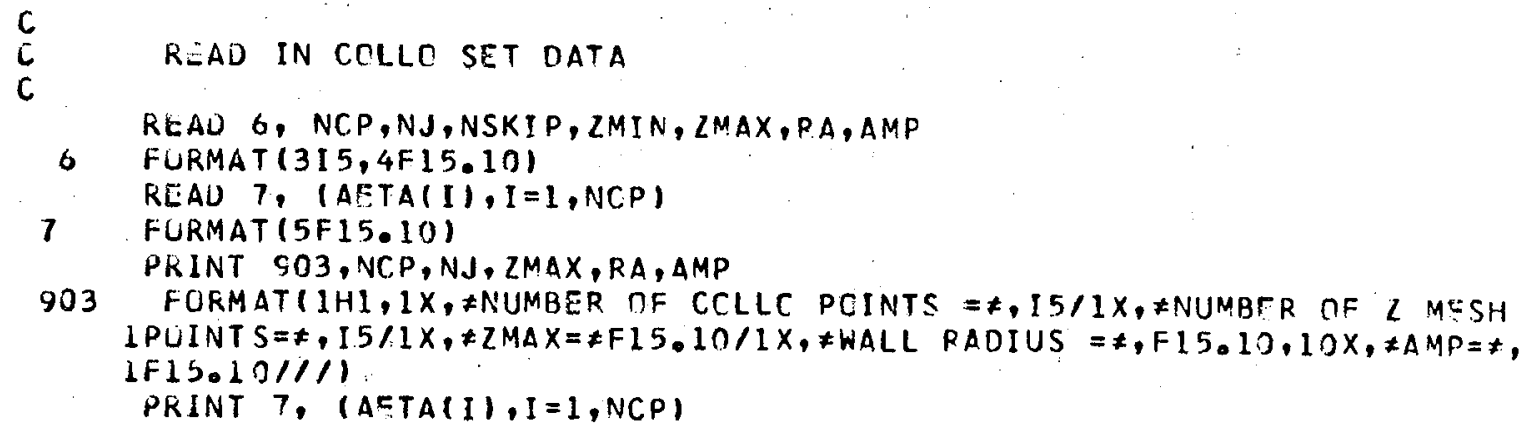

$\mathrm{C}$
$\mathrm{C}$
$\mathrm{C}$

START BAND CALCULATIONS

UEL $Z=(Z M A X-Z M I N) / F L O A T(N J-3)$ \& $N=2 * N C P$ \& $J=0$

DO $9 \quad I=1, N$

DO $9 K=1, N$

$Y(I, K)=0$.

9. $\quad X(I, K)=0$

$10 \quad J=J+1$ $\angle \approx O E L Z * F L D A T(J-2)+Z M I N$

$F_{W C}=F W(Z) \$ F W 1 C=F W L(2) \& F W 2 C=F W 2(Z) \& F W 3 C=F h 3(2)$

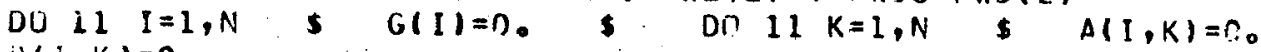

11

31 IF $(J-1) 12,12,14$

$120013 I=1, N C P$

$B(N C P+I, N C P+I)=1 \ldots \quad \$ \quad X(I, I)=-1$.

13

$X(N C P+I, N C P+I)=-1$.

CALL EAND(J) \$

IF $(J-A J) 16,18,18$

14 DU 17 IF $I=1, N C P$

$E T A=A F T A(I)$

FLAG $=1$. \$ CALL POLYITTA,NCP,FLAG, $, P 1, P 2, P 3, P 4)$

$T 2 A 2=-40 * E T A * F W C$

$T 1 A 3=60 * F T A T A * F H C * F W C$

$T 2 A 3=6 . * T A *(F W C * ?-F W 1 C)$

$T 3 A 3=2 . / R W(Z) \neq \# 2$

$T 1 A 4=-4 \cdot * E T A * F W C *(F T A * E T A * F W C * 2+1 \cdot / R W(Z) * 2)$

T $2 A 4=12, *$ F TAETA*FWC* (FW1C-FWC**2)

$T 3 A 4=4 . * C T A(3 . * F W C * F W 1 C-F W C * * 2-F W 2 C)$

$T 4 A 4=4$. $* W C / R W(Z) * * 2$

$T \angle A 5=($ FTA $A W C) * 4+2 . *(E T A * F W C / R W(Z)) * 2+10 / R W(Z) \neq * 4$

$T 2 A 5=60 *(F T A * 3) *(F W C * 2) *(F W C * * 2-F W 1 C)+(2 . * E A / R W(Z) * 2) *(4$ * *FW * $1 * 2-F W 1 C)-20 /(E T A * R W(2) * 4)$

T 3A5 = FTA*?TA*17.*FWC*4+4.*FWC*FW2C+3.*FW1C**2-18**FW1C*FWC* $1 * 2)+3 . /($ ITA*RW $(Z) * 2) * 2+2 . *(F W C * 2-F W(C) / R W(2) *$ ?

$T 4 A 5=E T A *(F W C * * 4+40 * F W C * F W 2 C+30 * F W 1 C * * 2-60 * F W 1 C * F W C * 2-F W 3$

$I C)-30 /(F T A *(F T A * R W(Z) * 2) * 2)-2 * *(F W C * 2-F W 1 C) /(F T A * R W(Z) * 2)$ $H i=-240 *\left(70 *(E T A * F W C) * 4-60 * F W 1 C *(F W C * E T A * 2) * 2+10_{0} *(F W C * 5 T A)\right.$ $1 R W(2)) * 2-2$ - *FWIC*(ETA/RH(Z))*2)

$H 2=(28 . *(F T A * F W C * 2) * 2+16$ * *FC*FW2C*ETA*2+2+12。*(FTA*FWIC)*2 $1-72$ - *FWIC*(FTA*FWC)*2*8**(FWC**2-FWIC)/RW(Z)**2)*(1.-30*ETA** 22)

$H 3=140 *(E T A * F W C * 2) * 2+160 * F h C * F W 2$ *ITA**2+12.*(ETA*FW1C)*2 $1-240 * F W I C *(E T A * F W C) * 2-4$. *FWC*FTA*2-8. 2 (FWC*2-FWIC)/RW(Z) $3 * 2) *(1,-E T A * 2)$.

$G(I)=H I+H 2+H 3$ \$ $G(I)=G(I) * D E(Z * 2$ 
$G(I)=-C(I)$

DO $17 . K=1, N C P$

$H=H T(E T A, P(K))$

$H I=H T 1(E T A, P(K), P 1(K))$

$H 2=H T 2(E T A, P(K), P 1(K), P 2(K))$

$H 3=H T 3(F T A, P(K), P 1(K), P 2(K), P 3(K))$

$H 4=H T 4(E T A, P(K), P 1(K), P 2(K), P 3(K), P 4(K))$

$A A 1=H$

$A A 2=T 1 A 2 * H 1$

$A A 3=T 3 A 3 *(H 2-H 1 / E T A)+T 2 A 3 * H 1+T 1 A 3 * H 2$

$A A 4=T 4 A 4 *(H 1 / E T A-H 2)+T 3 A 4 * H 1+T 2 A 4 * H 2+T 1 A 4 * H 3$

$A A 5=T 4 A 5 * \mathrm{H} 1+T 3 A 5 * H 2+T 2 A 5 * \mathrm{H} 3+T 1 A 5 * \mathrm{H}_{4}$

$A(I, K)=-D E L Z * A A 4 / 2$.

$A(1, N C P+K)=-D E L 2 * A A 2 / 2$

$I F(I, E G \cdot K) \quad A(N C P+I, K)=1$ 。

$B(I, K)=A A 5 * D E L Z * * 2$

$B(I, N C P+K)=A A 3 * D E L Z * 2-2 . * \triangle A L$

IF $\left(I . E Q_{0} K\right) \quad B(N C P+I, K)=-2$ * IF $\left(I_{0} F Q_{0} K\right) \quad B(N C P+I, N C P+K)=-D E L Z * 2$

$D(1, K)=D E L Z * A A 4 / 2$.

$D(1, N C P+K)=D E L Z * A A 2 / 2$ - $\triangle A 1$

17

$I F(I, F G \cdot K) \quad D(N C P+I, K)=1$.

CONTINUF

CALL EAND(J) \$ GO TO 10

DO $19 I=1, N C P \$ B(I, I)=-1 . \quad B(N C P+I, N C P+I)=-1, \quad Y(I, I)=1$. $Y(N C F+I, N C P+I)=1$.

CALL BANOIJ)

$N J=N J-1$

C

PRINT CUT A FUNCTIONS

DO $20 J=1, N J \& Z=Z M I N+D F L L * F L D A T(J-2)$

PRINT $21,2,(C) I, J), I=1, N C P)$

21 FURMAT $(1 X, F 8.5,5 X, 5(3 x, 515.8))$

CONT INUE

$c$

CALCULATE VELOCITY AND STREAM FUNCTICN DO $70 \mathrm{~J}=2$, NJ, NSKIP

$Z=Z M I N+D E L Z * F L O A T(J-2) \quad$ \$ RWALL=RW(Z)

PRINT 99, Z,RWALL

99 FORMAT $(/ /, 1 X, \neq Z=\neq, F 10.5,5 X, \neq R W A L L=\neq, F 10.8)$

PRINT 100

100 FURMAT(10X, $\neq R \neq, 15 X, \neq V Z *, 25 X, \neq V R \neq, 28 X, \neq V X I \neq, 30 X, \neq P S I+\neq, 1)$

$c$
$c$
$c$

DO $65 I=1,11$ \& ETA=。1*FLCAT(I-1)

STORE A(J) IN DP IN P4 ARRAY

DO $50 \quad K=1, N C P$

$50 \quad P 4(K)=C(K, J)$

$\mathrm{c}$

C SET UP ARRAYS

c

C STORE A-PRIME ARRAY IN P 3 AS OP DO $55 K=1$, NCP

$55 \quad P 3(K)=(C(K, J+1)-(K, J-1)) /(2, * D E L Z)$

$\mathrm{C}$

C SET UP H-PRIMEIETA ARRAY AND H/FTA ARRAY,STCRE IN A2,AL

C CALC ANO STORE H ARRAY 


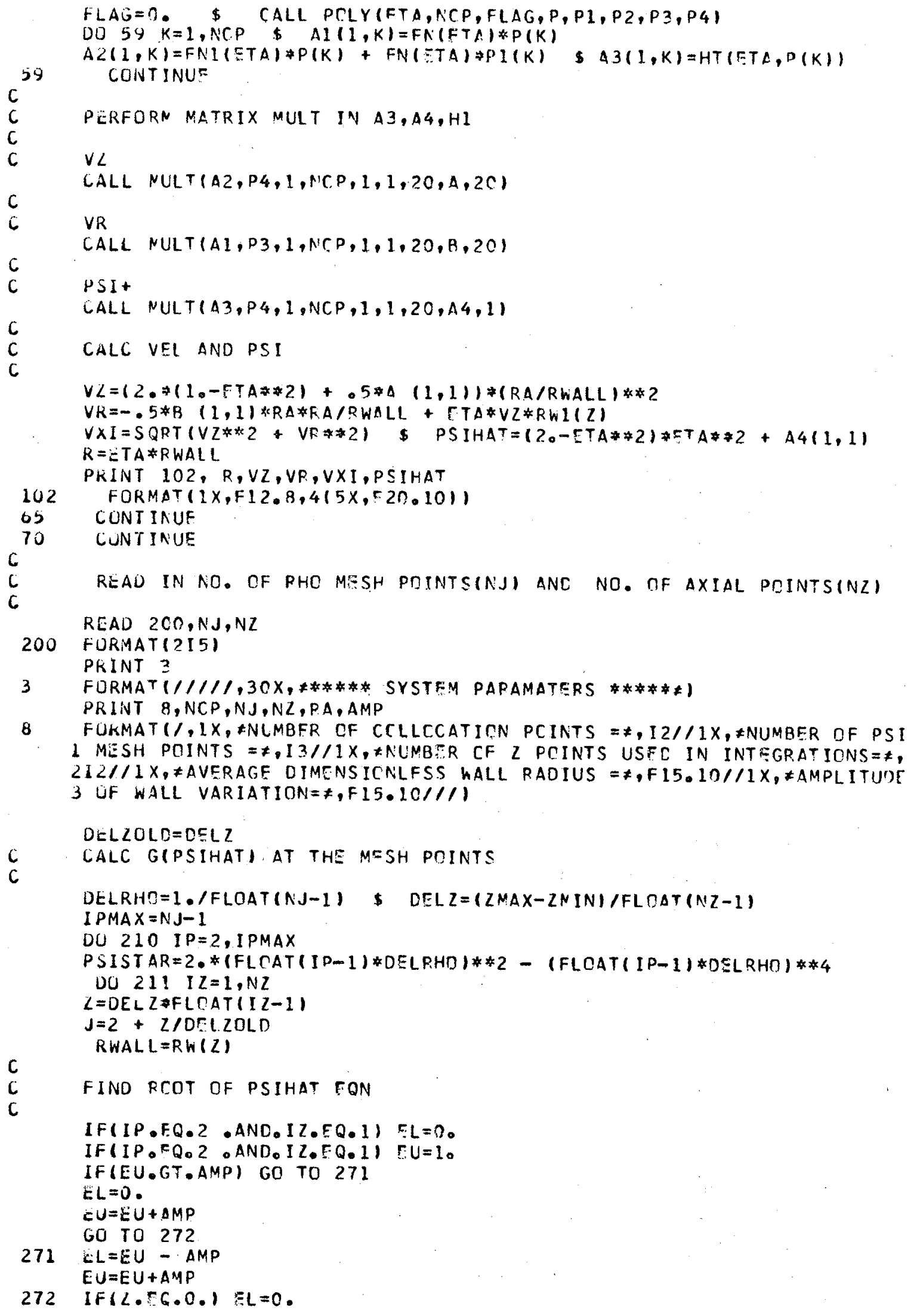




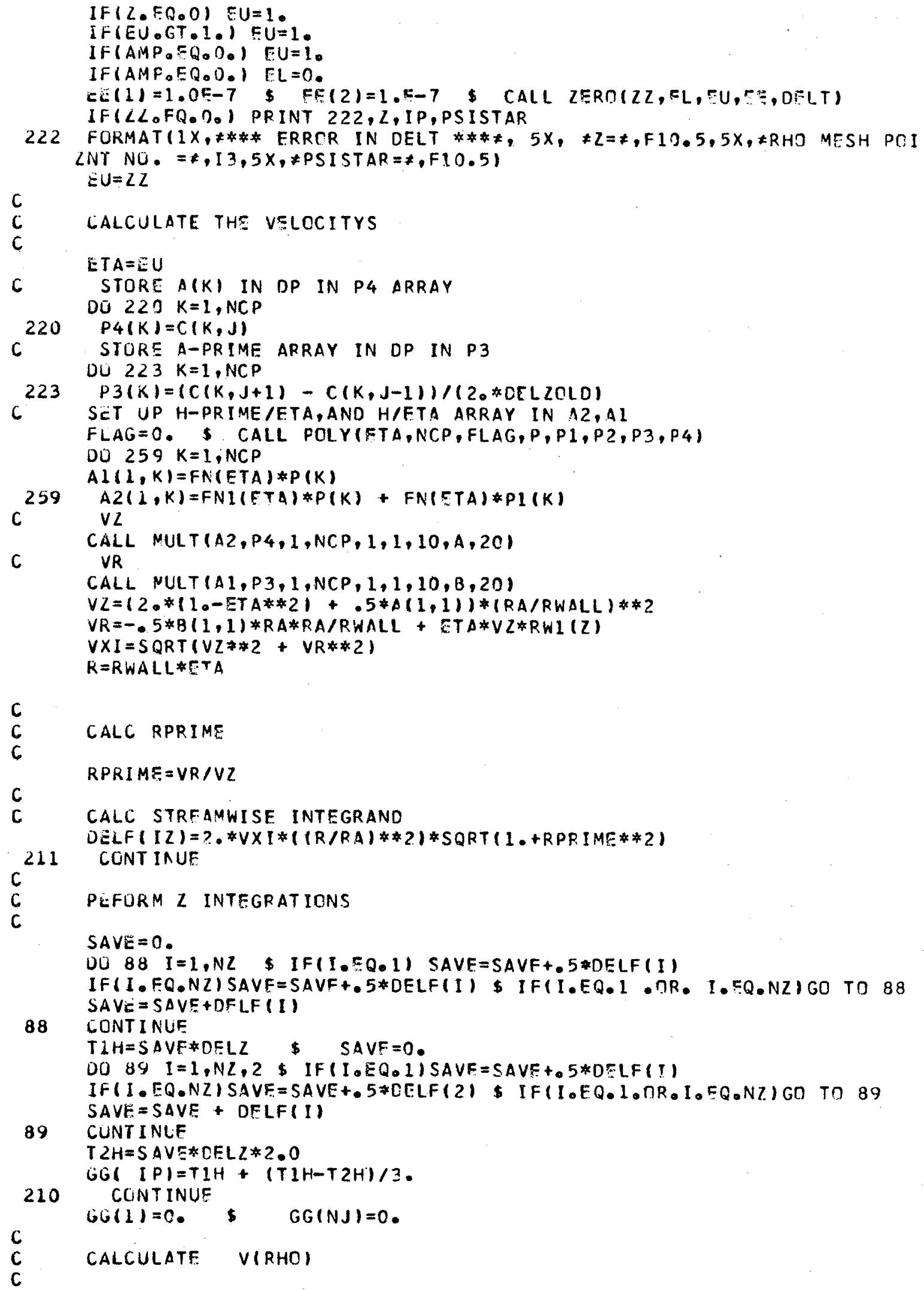




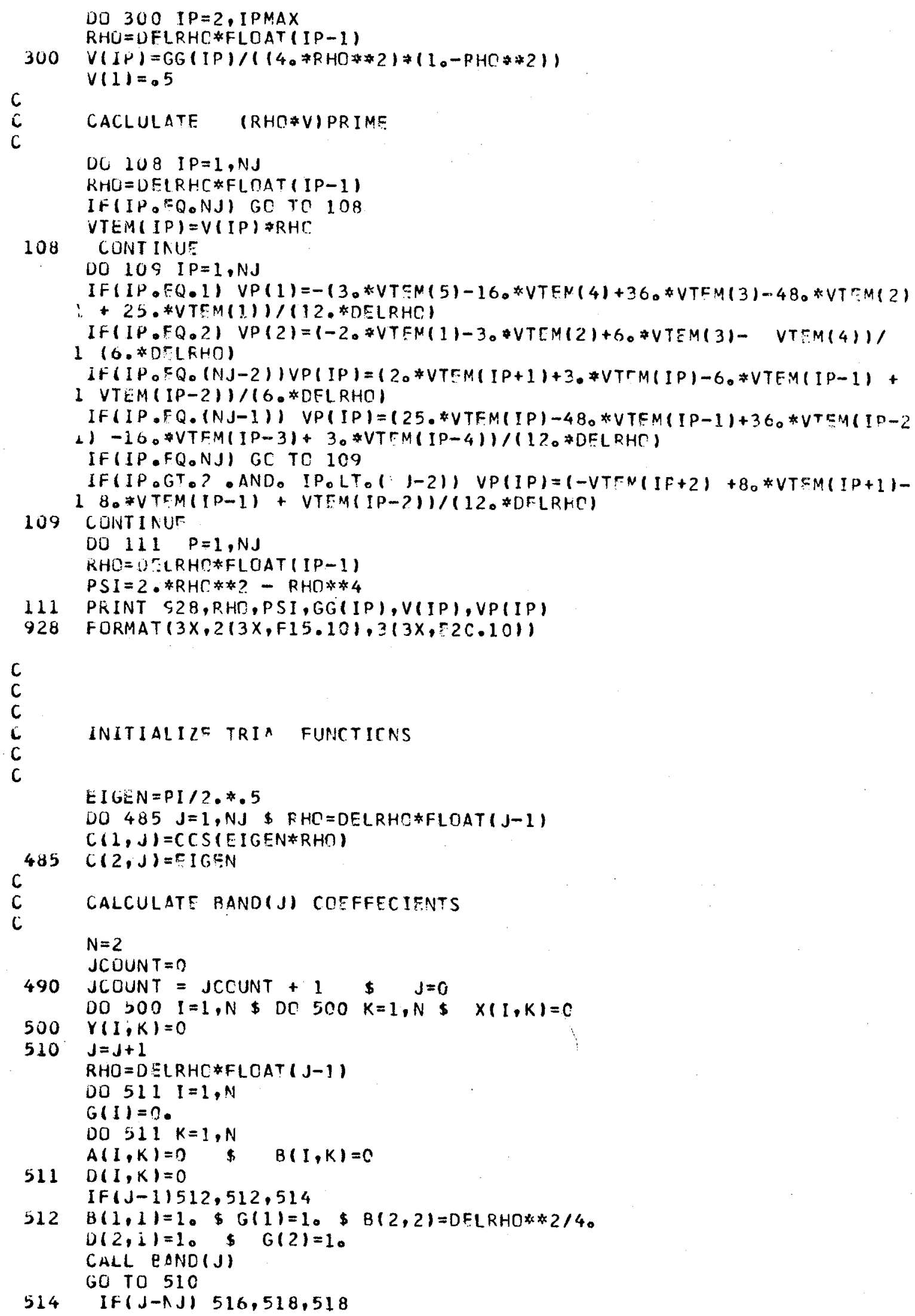


$516 A(1,1)=V(J)-V P(J) * D F L R H C /(2, * R+O)$

$B(1,1)=-20 * V(J)+40 * E I G E N *(10-R H C * 2) * D F L R H C * 2$

$B(1,2)=4$. $*(1, J) *(1,-R H C * * 2) * C E L R H O * * 2$

$D(1,1)=V(J)+V P(J) * D E L R H C /(2, * R H O)$

$G(1)=40 * E I G E N * C(1, J) *(1,-R H C * 2) * D F L R H C * 2$

$B(2,2)=-1$ \& $(2,2)=1$ \& CALL BAND(J) \$ GC TC 512

c

$B(1,1)=1$. \$ $A(2,2)=1, \$ E(2,2)=-1$. \$CALL BAND(J)

TEST FCR CCNVERGENCF

$E O=E I G E N \$ E I G E N=C(2,2)$

IF(ABS(EN-EIGEN) -LT, 10CF-10*ABS(EIGEN) GC TC 522

IF ( JCCUNT-10) $490,490,521$

\section{PRINT 608}

608 FORMATIFTHE RUN DID NOT CCNVERGE*)

522 PRINT 609, JCOUNT, FIGEN

609 FORMAT $\left(3 X_{1} \neq I\right.$ ITERATION COUNT $=\neq, I 2,10 X, \neq E I G$ SNVALUT $\left.=\neq, 220.10\right)$ $C P W=(C(1, N J-2)-40 * C(1, N J-1)+3 * * C(1, N J) / / 12 . * D E(R H O)$ PRINT $611, \mathrm{CPW}$

611 FORMAT $(/ /, 5 X, \neq C P R I M E$ AT THS WALL= $= \pm 16,8)$

999 CONTINUE:

CALL EXIT \$ TND

SUBROUTINE BANC(J)

DIMENSICN $A(20,20), B(20,20), C(20,103), C(20,41), G(20), \times(20,20)$,

$1 E(20,21,103), Y(20,20)$

COMMON $A, B, C, D, G, X, Y, N, N J$

101 FDRMAT (15HODETERM=0 AT $J=$, I4)

IF $(J-2) \quad 1,6,8$

$1 N P 1=N+1$

DO $2 \quad I=1, N$

$D(I, 2 * N+1)=G(I)$

$002 L=1, N$

$L P N=L+N$

$2 D(I, L F N)=X(I, L)$

CALL MATINV $(N, 2 * N+1$, DETERM)

IF (DETFRM) $4,3,4$

3 PRINT 101, J

$4005 K=1, N$

$E(K, N P 1,1)=D(K, 2 * N+1)$

DO $5 L=1, N$

$E(K, L, L)=-D(K, L)$

$L P N=L+N$

$5 X(K, L)=-D(K, L P N)$

RETURN

6 DU $7 \quad I=1, N$

DO $7 \mathrm{~K}=1, \mathrm{~N}$

DO $7 \mathrm{l}=1, \mathrm{~N}$

$7 D(I, K)=D(I, K)+A(I, L) * X(L, K)$

8 IF $(J-A J) 11,9,9$

9 DO $10 \quad I=1, N$

DO $10 \quad \mathrm{~L}=1, \mathrm{~N}$

$G(I)=G(I)-Y(I, L) * C(L, N P I, J-2)$

DO $10 \mathrm{~N}=1, \mathrm{~N}$

$10 A(I, L)=A(I, L)+Y(I, M) * E(M, L, J-2)$

11 DO $12 \quad I=1, N$

$D(I, N P I)=-G(I)$

DO $12 L=1, N$

$D(I, N P 1)=D(I, N P 1)+A(I, L) * F(L, N P 1, J-1)$

DU $12 K=1, N$

$12 B(I, K)=B(I, K)+A(I, L) * E(L, K, J-1)$

CALL MATINV (N,NPI, DFTERN)

IF (OETFRM) $14,13,14$

13 PRINT 101, J 


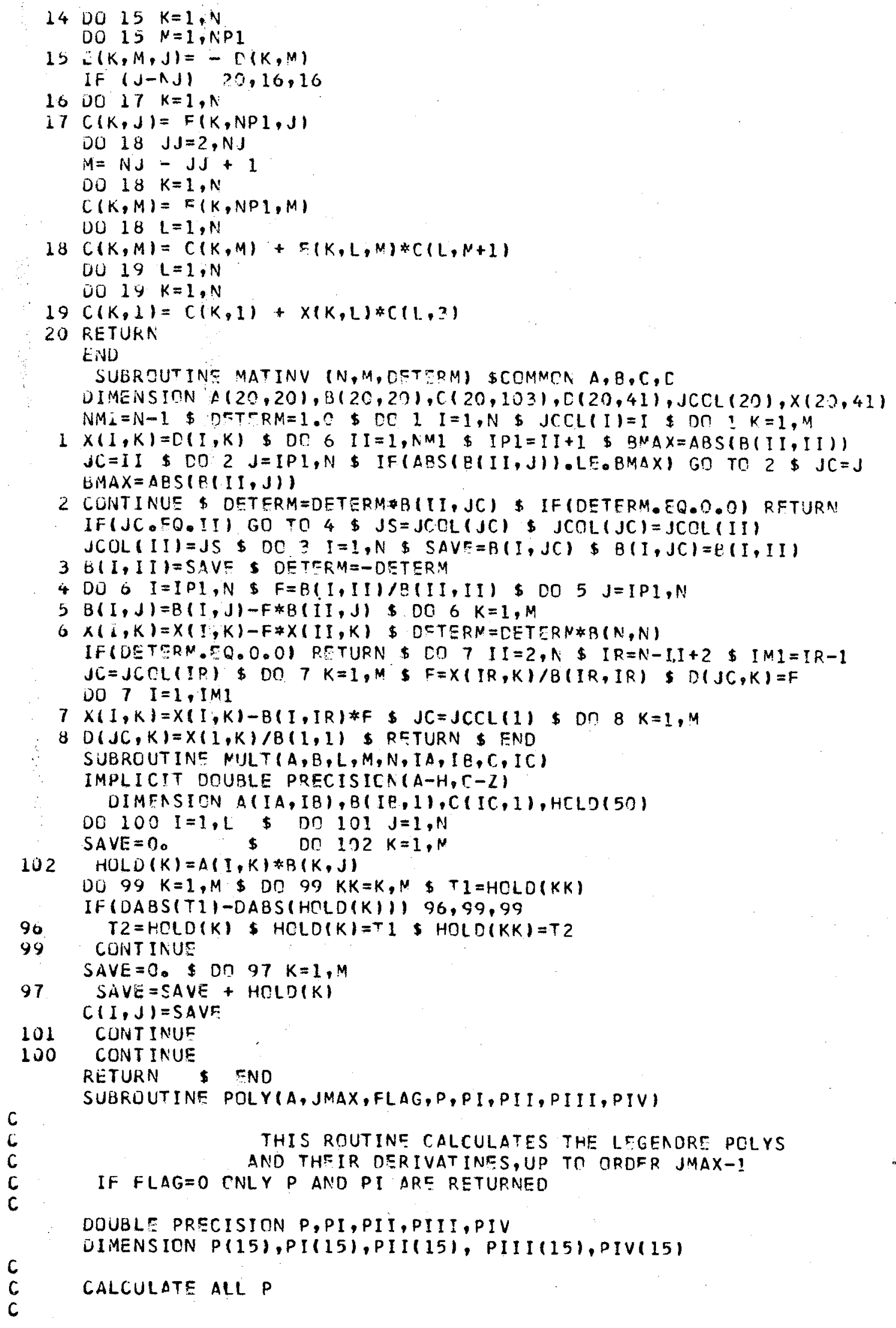




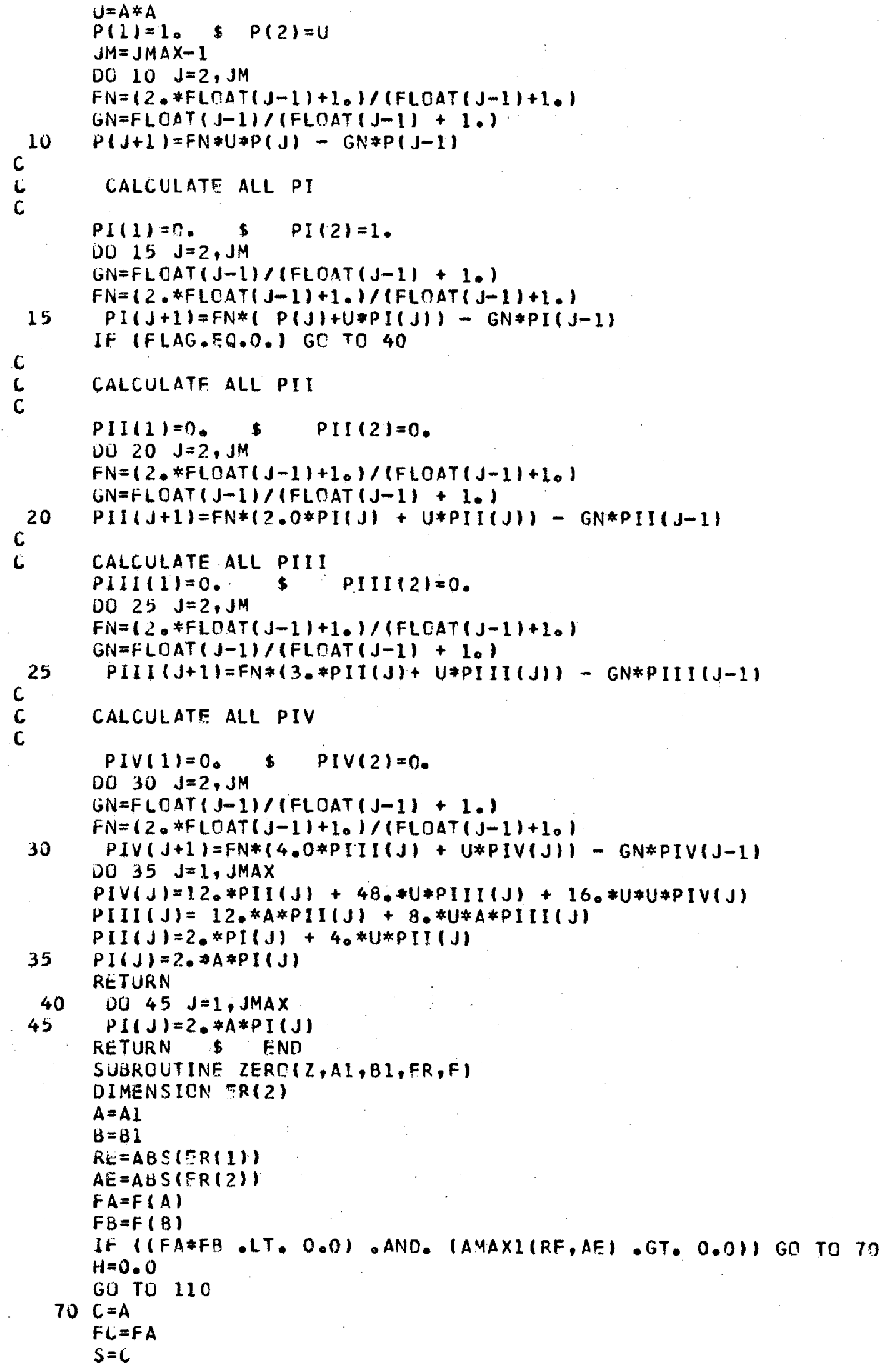




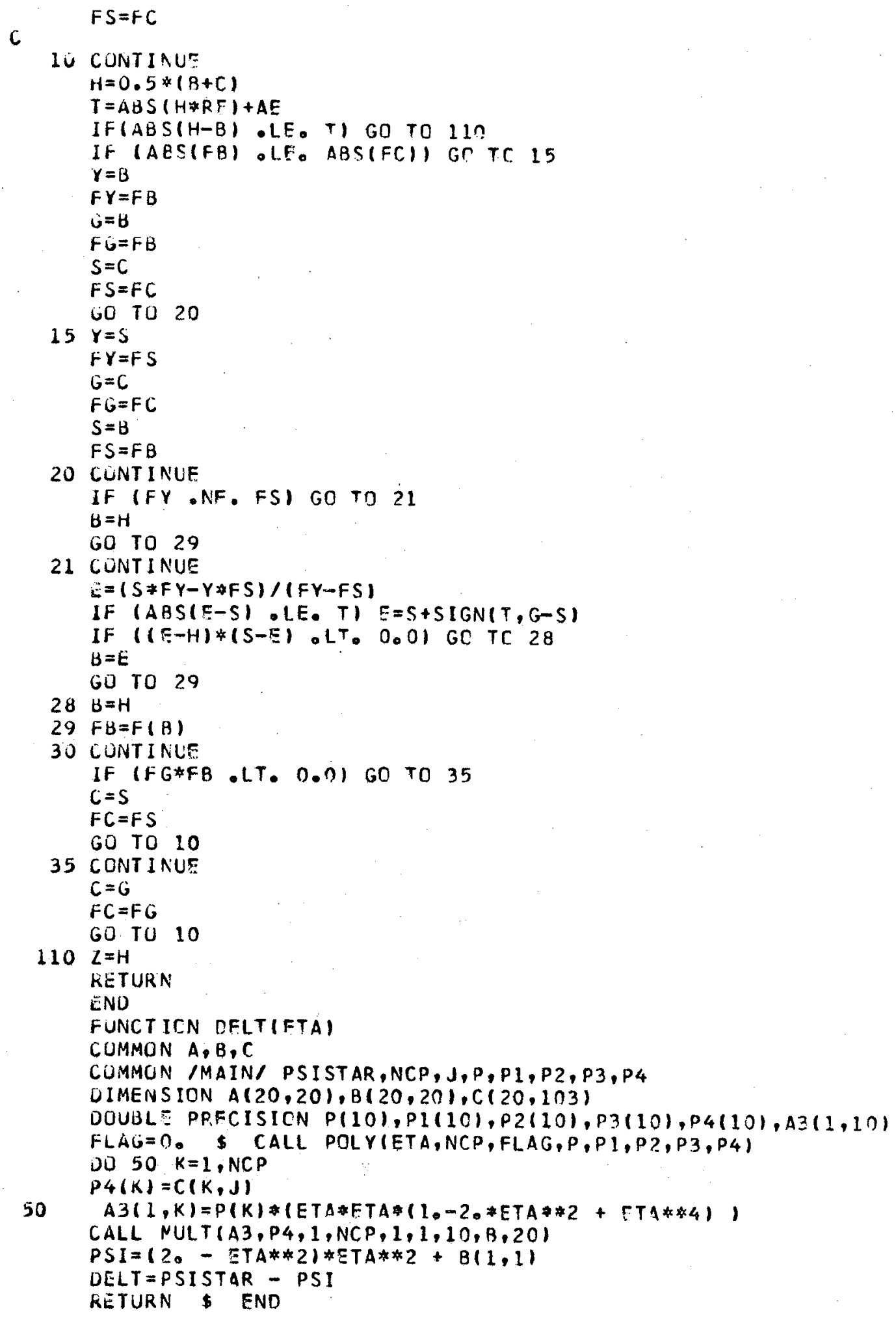


Program PDROP

This program evaluates the pressure drop in a sinusoidal PCT by integrating the z-component of the Navier-Stokes equation along the centerline. The results of this integration are used in equation 3.10 to evaluate the friction factor, Reynolds number product. The input parameters are identical to those of program INTER except the parameter NZ is not used. The subroutines BAND, MATINV and POLY are required. These are listed in INTER. 
PROGRAM PDROP (JUNK, INPL:T, CUTPUT)

DIMENSION DELF (103), GG(201),V(201),VP(2n1),VTEM(2ח1),ER(2)

DIMENSION $P(10)$,

$1 P 1(10), P 2(10), P 3(10), P 4(10), A 1(1,10), 02(1,10), 13(1,10), 44(1,1)$,

$2 C(20,103), G(20), A(20,20), B(20,20), D(20,41), X(2), 20), Y(20,20)$,

3ALTA $(10)$

OUUBLE PRFCISICN P,P1,P2,P3,P4,PT, PT 1,PT2,PT3, PT H,H,H1,H2,H3, H4,

$1 \mathrm{HT}, \mathrm{HT} 1, \mathrm{HT} 2, \mathrm{HT} 3, \mathrm{HT} 4, \mathrm{HNLD}, \mathrm{A} 1, \mathrm{~A}, \mathrm{~A} 3, \mathrm{A4}$

DOUBLE PKFCISICN FW, FWL, FH2,FW3, FWC, FW1C,FW2C,FW3C, T1 12, T1.A3,

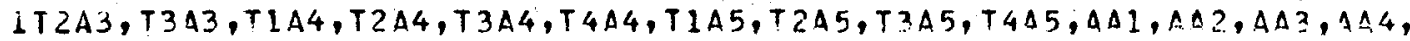
$2 A A 5$

$\mathrm{C}$
$\mathrm{C}$

COMMON A, B, C,D,G,X,Y,N,NJ

SET UP ALL FUNCTINN STATEMENTS

$R w(L)=R A-A M P * \operatorname{COS}(20 * P I * L)$

$R W I(Z)=2 * P I * A M P * S I N(2 * P I * Z)$

$R W 2(Z)=4$ **AMP*CCS $(2 * P I * Z) * P I * P I$

$R W 3(L)=-8_{0} * A M P * \operatorname{SIN}\left(2_{0} * P I * Z\right) * P I * 3$

$R W 4(\angle)=-16 * A M P * \operatorname{CCS}(2 * * P I * Z) * P I * 4$

$F(E T A)=F T A * E T A *(1, C-2 \cdot \cap * F T A * 2+F T A * 4)$

$F 1(E T A)=20 * T A *\left(10-40 * T A * 2+3_{0} * F T A * 4\right)$

$F 2(E T A)=2.0-6 . * E T A F T A *(40-5 * F T A * 2)$

$F 3(E T A)=-24 . * T A *(2.0-5 . * T A * 2)$

$F 4(E T A)=240 *(150 * T A * * 2-20)$

$F N(E T A)=E T A *(1.0-2.0 * F T A * 2+C T A * \$ 4)$

FNi $(E T A)=2.0 *(1.0-14 \cdot-3 \cdot * T A * 2) * T \cdot T * 2)$

$F W(Z)=R W 1(2) / P W(Z)$

FWI $(2)=R W 2(Z) / P W(Z)-F W(Z) * 2$

$F W 2(Z)=R W 3(Z) / R W(Z)-3 * F W(Z) * R W 2(Z) / R W(Z)+20 * F W(Z)$

$F W 3(Z)=(R W 4(Z)-4 * * W(Z) * R W 3(Z)-3$ * *FW $)(Z) * R W 2(Z)+30 * R W 2(Z) * F W(Z) * 2$

$1+6 . * R W(Z) * F W(Z) * F W(Z) * * 21 / R h(Z)$

$H T(E T A, P T)=F(F T A) * P T$

HTIISTA,PT,PT I I=FI(ETA)*PT \& F(ETA) *PT 1

HT2 (ETA,PT, PT $1, P T 2)=F 2(E T A) * P T+2 。 * F 1(E T A) \neq P T 1+F(E T A) \neq P T 2$

HT \& $(E T A, P T, P T 1, P T 2, P T 3)=F 3(C T A) * P T+3$ * * $2(E T A) * P T 1+3$ *F1(FTA)*PT2+

$1 \mathrm{~F}(\mathrm{ETA})$ *PT 3

HT4(ETA,PT,PT 1,PT2,PT3,PT4)=F4(ETA)*PT+4.*F3(ETA)\#PT1+6.*F?(ET (A)*PT $2+4 . * F 1($ ETA)*PT3 + F(ITA)*PT 4

C
C
C
$c$
$c$

$P I=3.141592654$

REAO IN CATA

READ NUMBER OF CTLLO SFT FOINTS TO BE FROCESSEO

REAO 5. NCS

FURMAT (I5)

or 999 III $=1$, NCS

C

C READ IN CCLLO SET DATA 
c

6

REAO 6, NCP,NJ, NSKIP, ZMIA, ZMAX, RA, AMP

FORMAT (3I5, 4FI5.10)

KEAD 7, (AETA 11$), I=1, N C P)$

FURMAT (5F15.10)

PRINT $903, N C P, N J, Z M A X, R A, A N P$

903 FURMATI///,1X, \#NUMERR CF COLLC PCINTS =*, I5/1X, NUMB:F OF $Z M T S H$

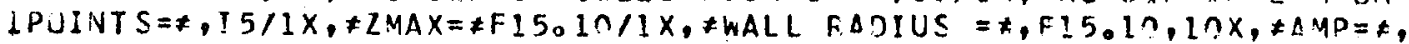
IFij.101/11

PRINT $7,(A R T A(I), I=1, N C, F)$

$\mathrm{C}$

$\mathrm{C}$

\section{START PAND CALCULATICNS}

DELL $=(2 M A X-Z M I N) / F L C A T(N J-3) \& N=2 * N C P \quad \$ \quad J=0$

DU $\rightarrow I=1, N$

DO $9 \quad K=1, N$

$Y(I, K)=n$.

$9 \quad x(I, K)=0$

$10 . J=J+1$

$Z=D E L Z * F L C A T(J-2)+Z M I A$

$F W C=F H(Z) \& F W 1 C=F W 1(L) \& F W 2 C=F W 2(L) \$ F W 3 C=F h 3(Z)$

OU $11 I=1, N \quad \$ \quad G(I)=0$ \& DC $11 K=I, N \quad \$ \quad A(I, K)=0$ 。

$B(1, K)=0$ 。

$110(1, K)=0$ 。

31 IF $(J-1) 12,12,14$

12 DO $13 I=1, N C P$

$B(N C P+I, N C P+I)=1$. \$ $\quad X(I, I)=-1$.

13

$X(N C P+I, N C P+I)=-1$ 。

CALL EANC(J) \$ GO TI 10

I $F(J-\wedge J) 16,18,18$

16 DU I7 $I=1, N C P$

$E T A=A T T A(I)$

$F L A G=1$. \$ CALL POLY(FTA,NCP,FLAG,P,PI, P2,P3,P4)

$T 1 A 2=-40 * F T A * F W C$

$T 1 A 3=6_{0} * F T A * F T A F H C * F W C$

$T 2 A 3=60 * 5 A *(F W C * 2-F W I C)$

$T 3 A 3=20 / R W(Z) * * 2$

T1A4=-4.*FTA*FWC*(FTA*FTA*FWC*2+1./RW(Z)*2)

$T \angle A 4=120 * F T A * F A * F W C *(F h 1 C-F W C * * 2)$

$T 3 A 4=40 * E T A * 130 * F W C * F W 1 C-F W C * 2-F W 2 C)$

$T 4 A 4=4 \cdot * F W C / R W(2) * 2$

$T 1 A 5=(F T A * F h C) * \# 4+2 . *(F T A * F W C / R W(Z)) * 2+1 . / R W(Z) * * 4$

$T 2 A 5=60 *(F T A * 3) *(F W C * 2) *(F W C * 2-F W 1 C) *(2 * * T A / P W(2) * 2) *(4$ * * FWC* $1 * 2-F W 1 C)-20 /(E T A * R W(2) * 4)$

T3A5 =ETA*ITA*17.*FWC**4+4.*FWC*F2C+3**FWIC**2-18*FW1C*FWC*

$1 * 2)+3.1(E T A * R W(2) * 2) * 2+2 * *(F W C * 2-F W I C) / R h(Z) * 2$

$T 4 A 5=5 T A *(F W C * 4+40 * F W C * F h 2 C+30 * F h 1 C * 2-60 * F W 1 C * F W C * 2-F W 3$

LC) - 3०/(FTA*(ETA*RW(Z)*2)*2)-2* (FWC*2-FWIC)/(*TA*RW(Z)*2)

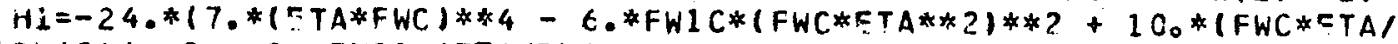

$1 R W(2)) * 2-2, * F W 1 C *(I T A / R W(Z)) * 2)$

$H 2=1280$ (FTA*FWC*21*2+16.*FW*FW2C*ITA**2+12.*1FTA*FW1C)*2 $1-720 * F W 1 C *(F T A * F W C) * 2+8 *$ (FWC*2-FWIC)/PW(2)*2)*(10-30*TTA** 221

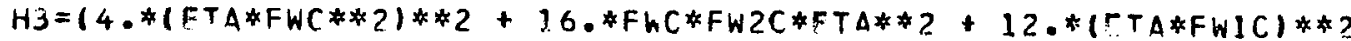
$1-240 * F W 1 C *(E T A * F W C) * 2-4$ * *W?C*FTA**2-8**(FWC*2-FW1C)/RW(Z) $3 * 21 * 11 \cdot-F T A * 21$

$\mathrm{G}(\mathrm{l})=\mathrm{H} 1+\mathrm{H}_{2}+\mathrm{H}_{3}$

$G(I)=-G(I)$

$\$ G(I)=G(I) * D E L I * * 2$

$0017 \mathrm{~K}=1, \mathrm{NCP}$ 


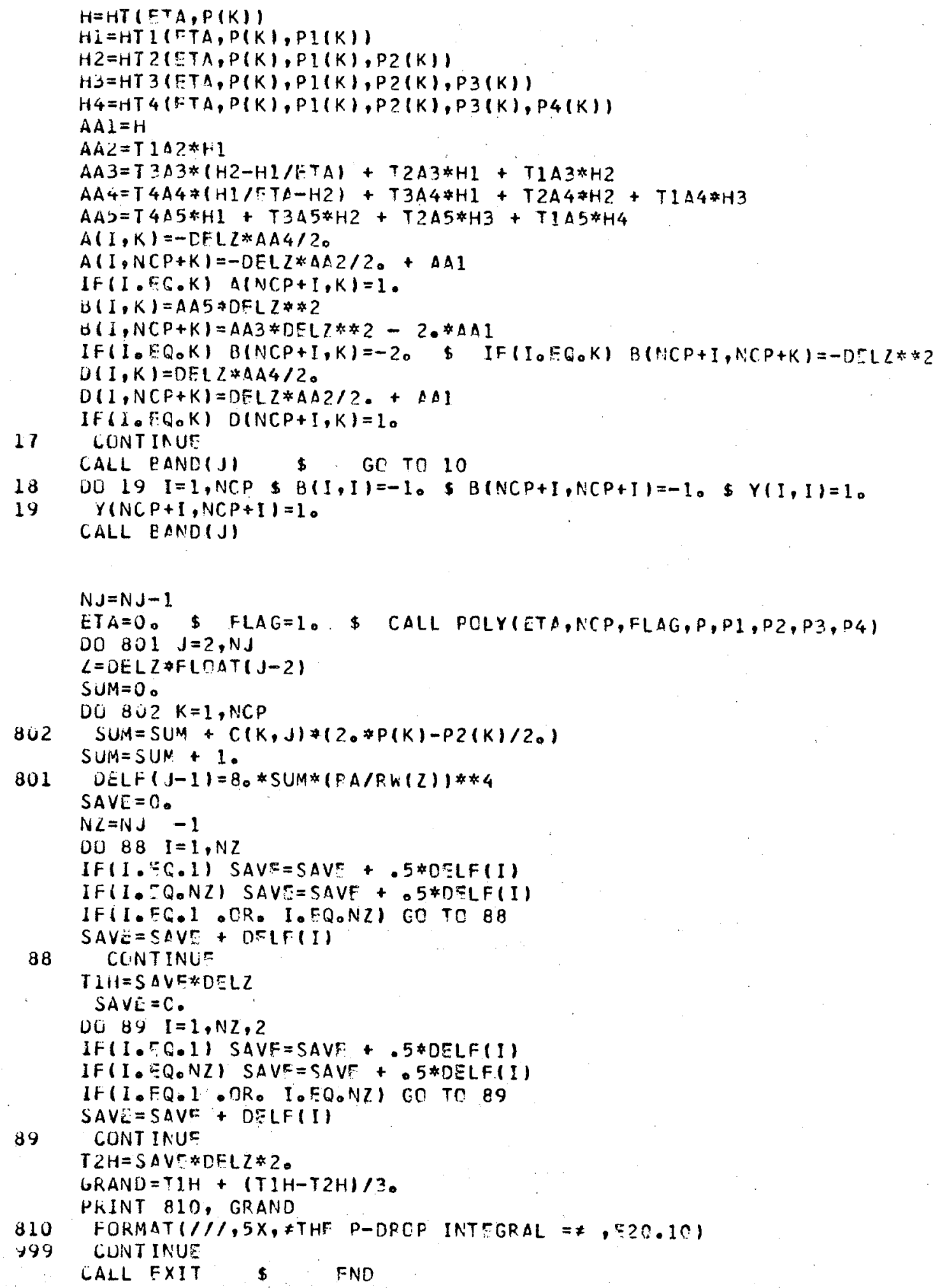


Program LOWPE

This routine calculates the first eigenvalue of Laplace's equation in a sinusoidal PCT as outlined in Chapter 5. Collocation is used in the $\eta$ coordinate and a finite-difference grid is established in the axial coordinate. The modified BAND routine is used. (See Appendix C.) The input parameters are: NPM, the number of parameter sets to be processed; NJ, the number of axial mesh points; RA and ARA, the average radius, and amplitude to average radius ratio; NCP, the number of $\eta$ coordinate collocation points. 
LEVEL $2, E$

JIMEASION! $A(31,31), B(31,31), D(31,63), F(1), 22,103), \times(31, ? !)$, $1 Y(31,31), G(31), B J C(15), B J C 1(15), B J C 2(15),(131,103)$, TA(15) LUMMUN / DUMMY / $F$ $C U$ IIMUN $A, B, C, D, G, X, Y, N, N J$

C SET UP STATEMENT FUNCTICAS

$R W(\angle)=R A *(1,-A R A * \operatorname{COS}(20 * P I * Z))$

$K W I(Z)=R A * 2 . * P I * A R A * S I N(2 * P I * Z)$

$R * 2(\angle)=R A * 4$ * $A R A * C C S(2 . * P I \neq Z) \neq P I * * 2$

$F(\angle)=120 * P I * A R A * S I N(20 * P I * 2) 1 /(10-A R A * C r S(20 * P I * Z))$

$F I(L)=-F(Z) * 2+140 * A R A * \operatorname{COS}(20 * P I * Z) * P I * 2) / 110-A R A * \operatorname{COS}(20 * P I * Z))$ RATIO $(Z)=1 . /(1 .-A R A * \operatorname{COS}(2, * P I * Z))$

$P I=3.141592654$

C READ IN CATA

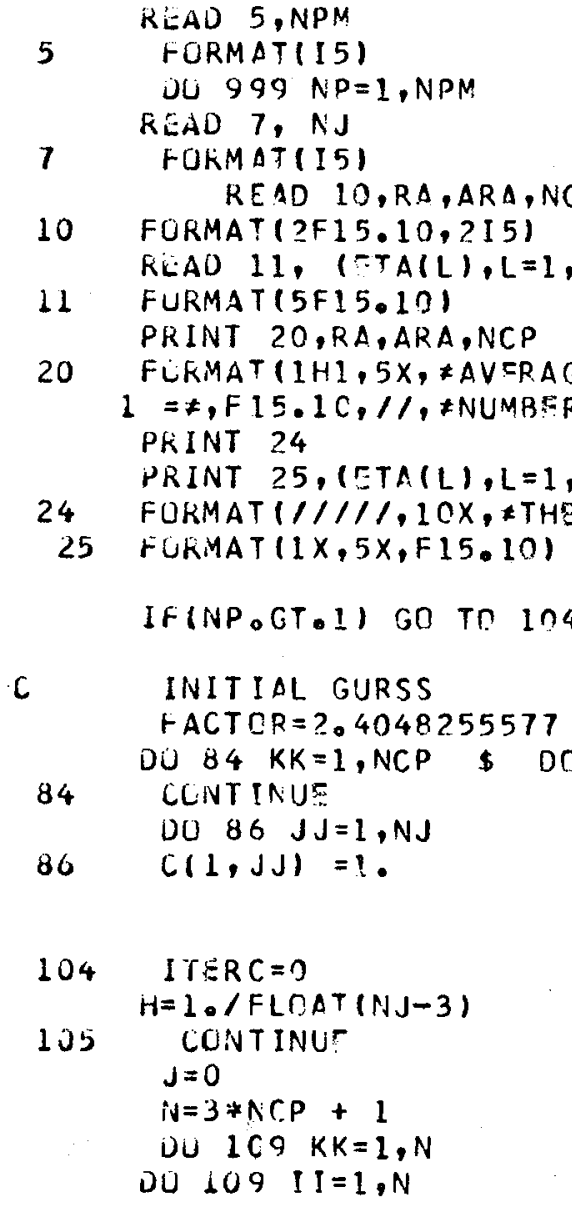




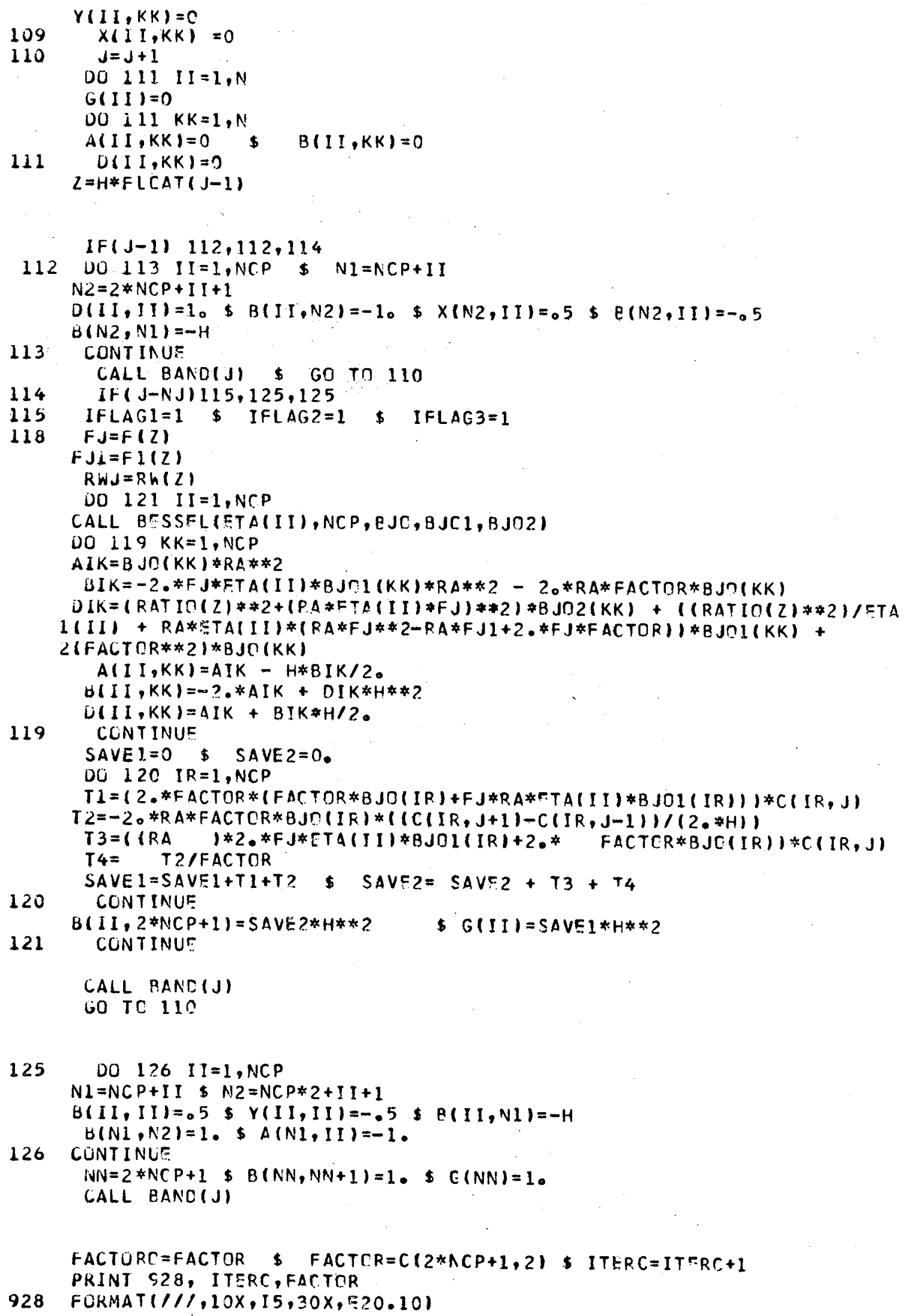


IF (ABSIFACTCR-FACTRRCIOLT。FACTCR

ITERMAX $=1$ ก

IF(ITFRC。CT。 ITERMAX) GE TC 225

iU TC 1.25

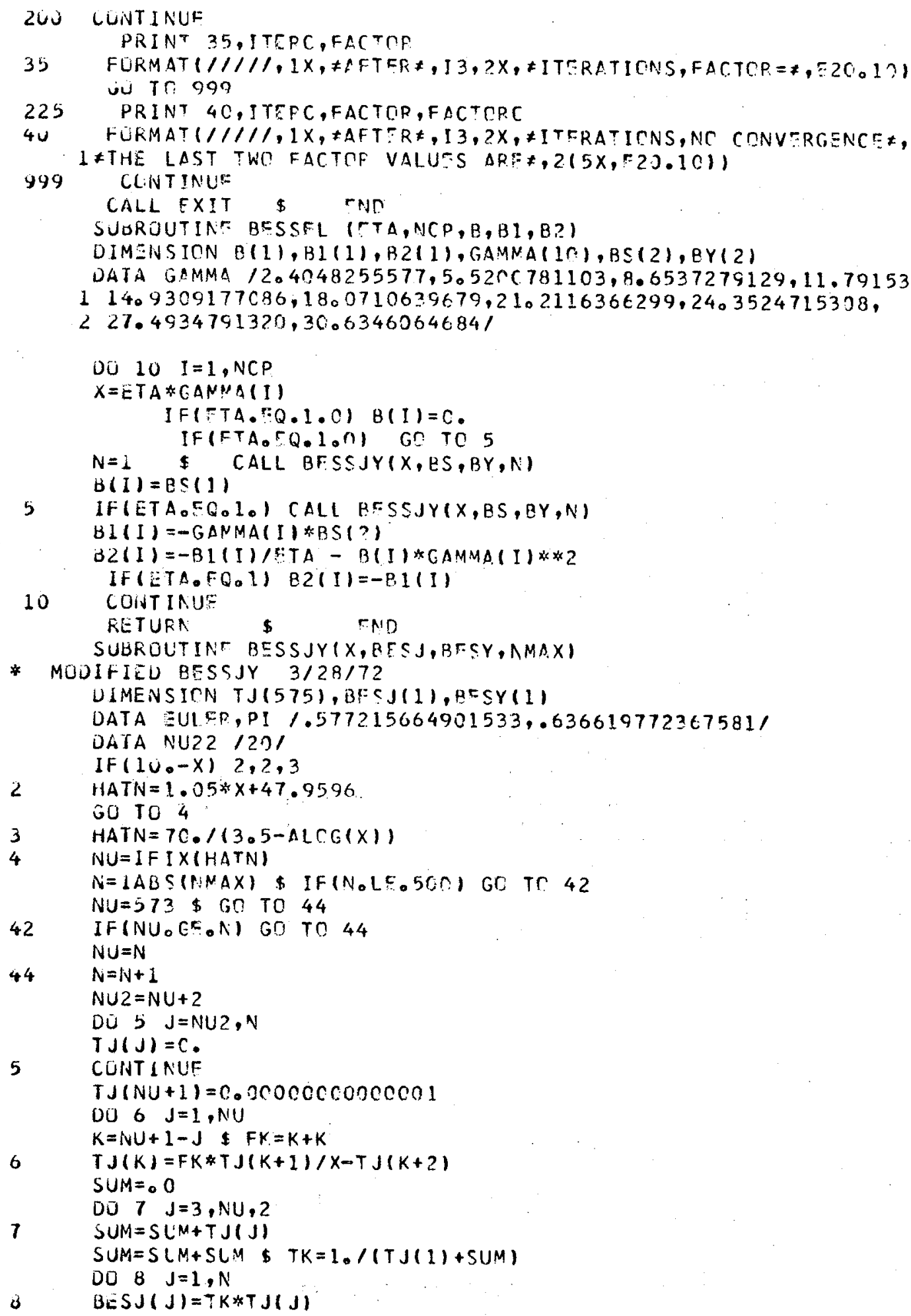




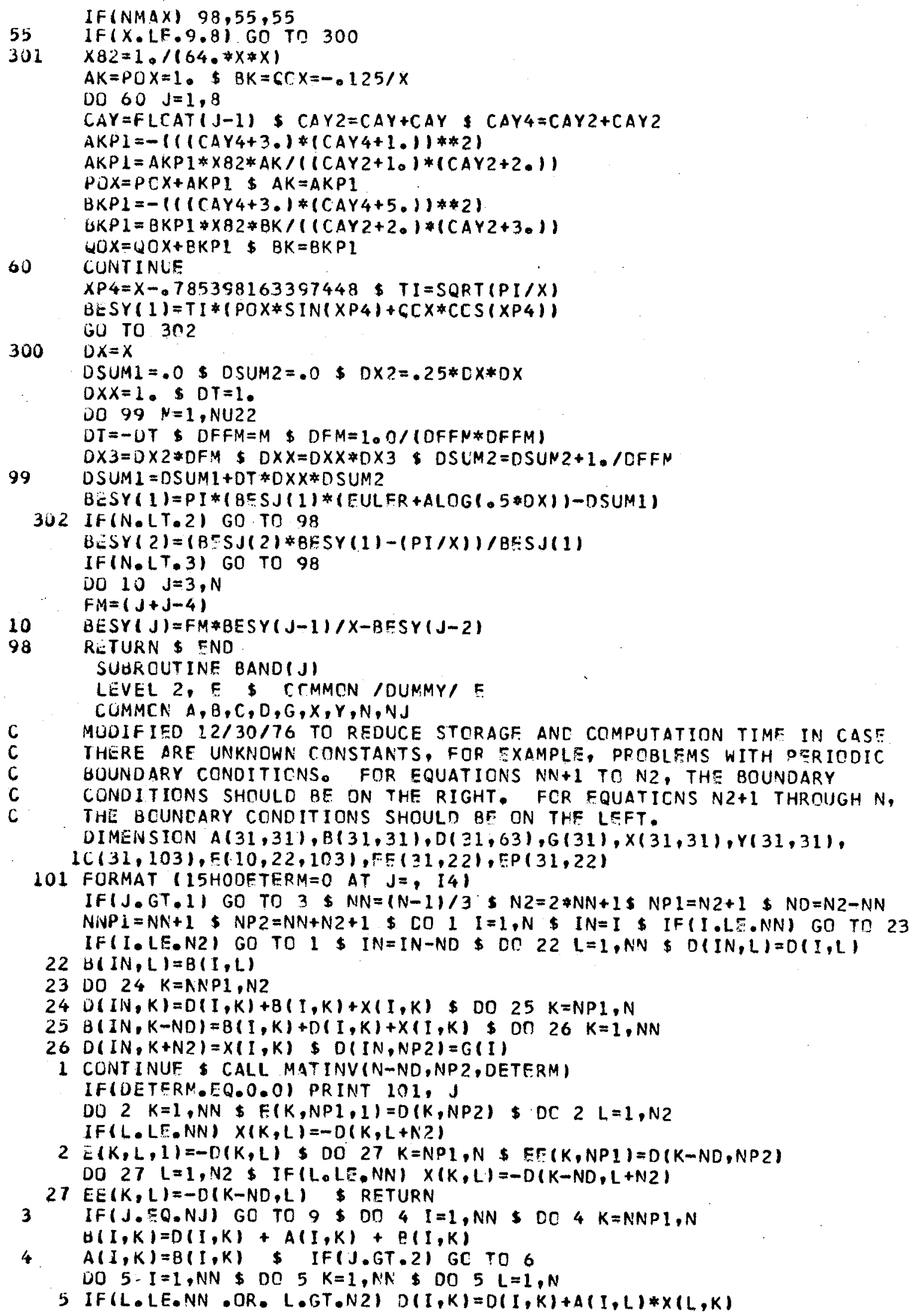


$60028 I=1, N N \$ D(I, N P !)=-G(I) \$ D C 7 L=1, N$

$I F(L, L E, N N) \quad D(I, N P I)=D(I, N P I)+A(I, L) \neq E(L, N P I, J-1)$

$I F(L \bullet G T \circ N 2) \quad D(I, N P 1)=D(I, N P 1)+A(I, L) * F(L, N P 1)$ \$ DO $7 \mathrm{~K}=1, N 2$

$I F\left(L \cdot L E_{0} N N\right) B(I, K)=B(I, K)+A(I, L) * F(L, K, J-I)$

$7 I F(L, G T \cdot N 2) \quad B(I, K)=B(I, K)+\Delta(I, L) * E(L, K)$ DO $28 K=N N P 1, N 2$

$17 E P(I, M)=E[(I, M) \$ D C \& K=1$, NN

$8 E(K, M, J)=-D(K, N) \$ D O 19 I=N P 1, N \$ D O 19 L=1, N P 1$

IF(L,GT,NN) GO TC $18 \$ F E(I, L)=0,0 \$ I F\left(J_{0} E_{0} Q_{0} 2\right) F E(I, L)=X(I, L)$

$180019 \mathrm{~K}=1$, NN

$19 E(I, L I=E F(I, L)+E P(I, K) * C(K, L, J) \&$ RETURN

$90012 \quad I=1, N 2 \$ D(I, I)=G(I) \& D C \quad 10 \quad L=1, N N$ $D(I, 1)=D(I, 1)-Y(I, L) * F(L, N P 1, J-2) \$ D O 10 K=1, N 2$

$10 A(I, K)=A(I, K)+Y(I, L) * E(L, K, J-2)$ \& DC $11 L=1$, NN

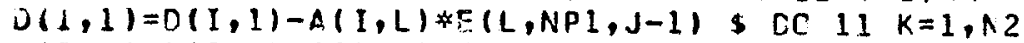

$11 B(I, K)=B(I, K)+A(I, L) * E(L, K, J-1) \$ D O 20 L=N P 1, N$ $A(I, L)=A(I, L)+Y(I, L)+B(I, L) \$ C(I, I)=D(I, I)-A(I, L) \neq E C(L, N P I)$ DO $20 \mathrm{~K}=1, \mathrm{~N} 2$

$20 B(I, K)=B(I, K)+A(I, L) * E E(I, K) \$ C O 12 K=1$, N2

$12 I F(K, G T, N N) B(I, K)=B(I, K)+Y(I, K)+A(I, K)$ CALL MATINVIN2,1, DFTERMI S IFIDETERM.EG.0.CI PRINT 101, J DO $13 K=1, N 2$

$13 C(K, J)=D(K, I) \$ D C 2 I I=N P I, N \& C(I, J)=E E(I, N P 1) \$ D O 2 I L=1, N 2$

$21 C(I, J)=C(I, J)+E E(I, L) * C(L, J) \$ D O 15 J J=2, N J \$ M=N J-J J+I$ $D O 14 K=1, N \$ I F(K \circ L E 0 N N) C(K, M)=E(K, N P 1, M)$

$14 I F(K, G T$.NN) $C(K, M)=C(K, M+1) \$ C C 15 K=1$, NN \$ DC $15 L=1, N 2$

$15 C(K, M)=C(K, M)+E(K, L, M) * C(L, M+1) \$ D O 16 K=1$, NN \$ DO $16 L=1$, NN

I6 $C(K, 1)=C(K, 1)+X(K, L) \neq C(L, 3)$ \$RETURN \$ END SUBRCUTINE MATINV (N,M,OETERM) \$COMMCN $A, B, C, O$ DIMENSION A $(31,31), B(31,31), C(31,103), C(31,63), J C C L(35), X(31,63)$ $N M I=N-1 \$$ OETERM $=1.0$ SOC $1 \mathrm{I}=1, \mathrm{~N} \$ \mathrm{JCCL}(\mathrm{I})=I \& D O 1 \mathrm{~K}=1, M$

$1 X(I, K)=D(I, K) \$ 006 I I=1, N M I \$ I P I=I I+1 \$ B M A X=A B S(B(I I, I I)$ $J C=I I \& D C 2 J=I P I, N$ \& IF(ABS(E(II,J)).LF,BMAX) GO TO $2 \$ J C=J$ $B M A X=A B S(B(I I, J))$

2 CONTINLE \$ DETERM=DETERM*B(II,JC) \$ IF (DETERM॰EQ.O.O) RETURN IF (JC.FQ.II) GO TO 4 \$S $=J C N L(J C) \$ J C \cap L(J C)=J C O L(I I)$ $J C C L(I I)=J S \$ D O \quad 3 \quad I=I, N \$ S A V E=B(I, J C) \$ B(I, J C)=B(I, I I)$

3 O $(I, I I)=S A V E \$$ OETERM $\$$ DETEFM

4 DU $6 I=I P I, N$ \$ $F=B(I, I I) / R(I I, I I) \$ C Q 5 \mathrm{~J}=I P I, N$

$5 B(I, J)=B(I, J)-F * B(I I, J) \$ D \cap 6 K=1, N$

$0 x(I, K)=X(I, K)-F * X(I I, K) \$ D E T E R M=D F T E R N * B(N, N)$ IFIUETRRM.RQ.0.0) RETURN \$ DC 7 II=2, $N \quad$ IR=N-II+? \& IMI=IR-1 $J C=J C O L(I R) \$ D O 7 K=I, M \$ F=X(I R, K) / B(I R, I R) \$ D(J C, K)=F$ DO $7 \quad I=1, I M I$

$7 X(I, K)=X(I, K)-B(I, I R) * F \$ J C=J C D I(1)$ \&D $g \quad K=1, M$

$8 D(J C, K)=X(1, K) / B(1,1) \$ R E T U R N \$ E N D$ 
Program LOWPEC

This program performs the identical calculations as LOWPE.

A double collocation procedure as given by equations 5.14 and 5.18

is used to approximate the solution. The input parameters are identical

to those of LOWPE. In addition, the number of Fourier terms, NFT, must be specified. The subroutines MATINV, BESSELL and BESSJY are required. These are listed in LOWPE. 


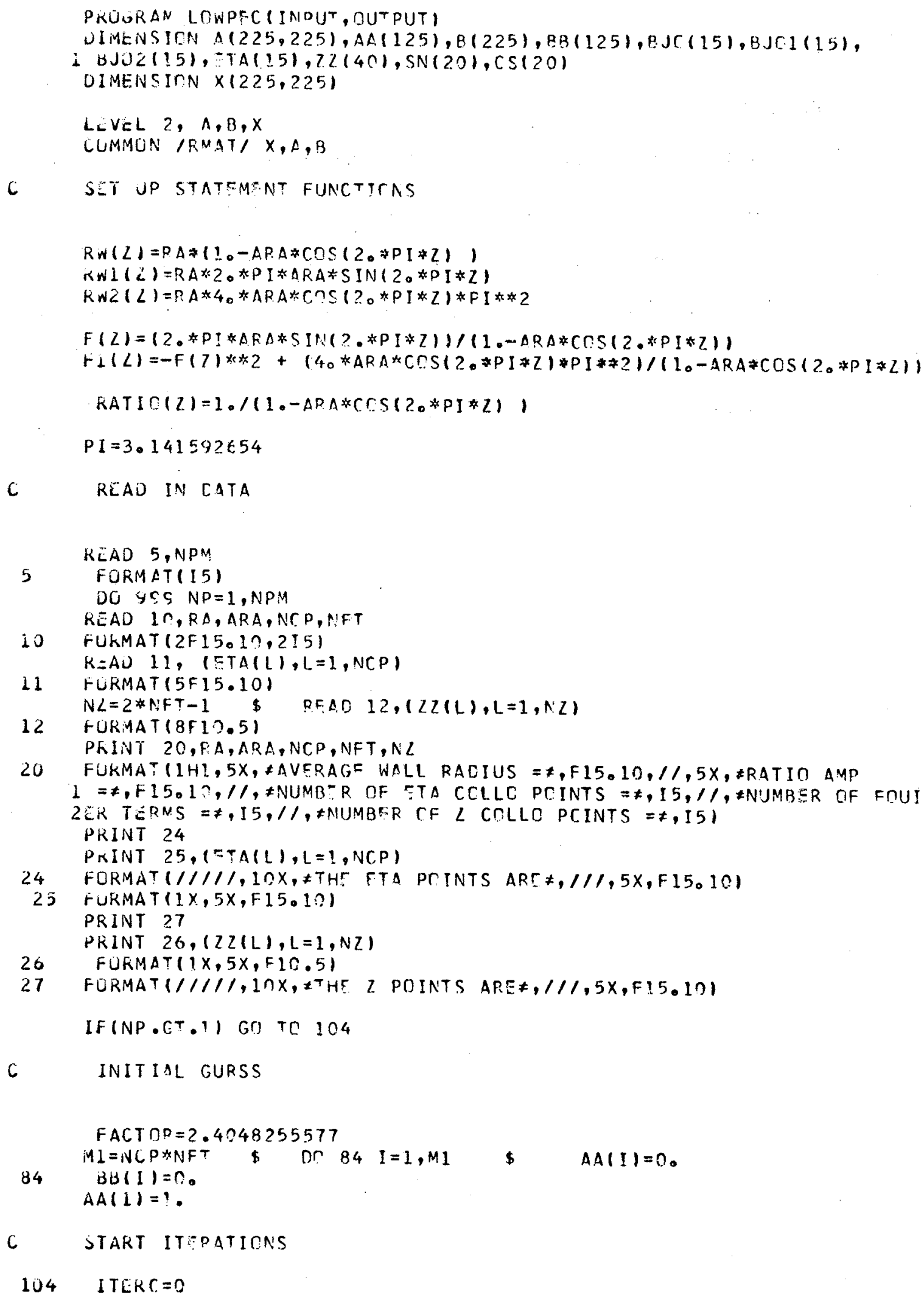


85 CONTINUE

$83 \quad A(1, J)=0$ 。

$M M=i N C P *(2 * N F T-1)+1$ \& DO $83 \quad I=1, M M$ \& DO $83 \mathrm{~J}=1, M M$

C ICETA IS THE FTA COLLP PEINT COUNTER

DO LUC ICFT $A=1$, NCP

CALL BESSFL (ETA(ICETA), NCP, BJO,BJOL, EJC2)

C II IS I Z CCLLC PIINT CCLNTER

$N S=2 * A F T-1$

DO 100 II $I=1$, NS

$\angle=\angle L(I I)$

CALL SUBSIN(Z,NFT, SN, CSI

C I IS THE LQUATICN NUMBFR CCUNTER

$1=(I C F T A-1) *(2 * N F T-1)+I I$

C ISA IS THE BELSEL FUNCTICN CCUNTER

$T L=(P A T I \cap(Z) * 2+(P A * F T A(I C P T A) * F(2)) * 2) * \operatorname{RT} 2(1 S A)+$ UO 90 I $S A=1, N C P$

1 (IRATIO(Z)*2)/TTAIICFTA) +RA*?TAIICFTA)*(RA*F(Z)*2-RA*FI(Z)

$2+20 * F(Z) * F A C T O P$ ) $* B J$ III ISA)

C IR IS THE FCUIFR COEFF CCUNTER.

LU $90 \quad$ IR $=1, N F T$

C

IRR IS THF J PCSITION CCUNTFR OF THE A MATRIX

$I R R=(I S A-1) * N F T+I R$

$T 2=T 1+(F A C T O R * 2-(2 * P I * R A * F L C A T(I R-1)) * 2) * B J C(I S A)$

$T 3=4$. $* P I * R A * F L C A T(I P-1) *(R A * F(Z) * E T A(I C E T A) * B J O 1(I S A)+F A C T D R * B J O(I$ (SA)

$A(I, I R R)=T 2 * C S(I R)+T 3 * S N(I R)$

IF (IR.EQ.I) GO TO 90

$A(I, N C F * N F T+I R F-I S A)=-T 3 * C S(I R)+T 2 * S A(I R)$

90 CINTINU:

$S A V E B L=0$ 。

SAVERTS=0.

C

IU IS THE BESSEL RCCT CCUNTEP.

DC 95 IU $=1, N C P$

T4=2. \#F(I) ETA(ICETAI*BJOI(IU)*RA +2。* FACTOR*BJO(IU)

$T b=2$. $* J C(I U) * F A C T C P * 2+2$. *F $\triangle F F(Z) * E T A(I C T T A) * F A C^{T} C R * B J O I(I U)$

C IV IS THE FCUITR TEFM CCUNTTR

UU 95 IV $=1, N F T$

$T O=4 . * F I * F L C A T(I V-1) * B J C(I U) * R A$

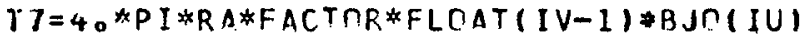

SAVEBL $=S A V E B L+(T 4 * C S(I V)+T 6 * S N(I V)) * A A((I U-1) * N F T+I V)+$

$1(T 4 * S N(I V)-T 6 * C S(I V)) * E E((I U-1) * N F T+I V)$ 
SAVERHS $=S A V E R H S+(T 5 * C S(I V)+T 7 * S N(I V)) * A A((I L-1) * N F T+I V)+$ $1(T j * S A(I V)-T 7 * C S(I V)) * B((I U-I) * N F T+I V)$

$A(I, N C F *(2 * N F T-1)+1)=S A V E B L$

B $(I)=S A V E P . H S$

\section{CUNTIAUE}

DU $101 K K=1$, NFT

$A(N C P *(2 * N F T-1)+1, K K)=1$ 。

101 CONTINLF.

$B(N C P *(2 * N F T-1)+1)=1$.

$N A=N C F *(2 * N F T-1)+1 \$$

IF (DETFPM॰FQ.0.) PF INT 30

30 FORMAT $(/ /$, IDFTERM FQUALS ZFRC*)

IF (UETERM.EQ.O.) CALL EXIT

CALL MATINVINA, ?, CFTFRM)

DU 150 ISA $S$ I,NCP $\$$ DC 150 IR $=1$, NFT

$I R R=(I S A-1) * N F T+I R$

$A A(I R R)=P(I R R)$

$I F\left(I R_{0} F Q_{0} I\right) \quad B B(I P R)=0$ 。

$I F(I R \circ C T \circ 1) B B(I R R)=B(I K R+\Lambda C P * A F T-I S A)$

150 CONTINLE

ITERC $=$ ITFPC + 1

FACTCRO =FACTOR \$ FACTCP $=8(N A)$

PKINT 928, ITERC,FACTCR

$9 \angle 8$ FURMAT $1 / 1 /, 10 \mathrm{X}, 15,30 \mathrm{X}, \mathrm{E20.101}$

PRLNT 927, (AAII), BB(I), I =1, MI)

927 FORMAT $(10 X, 2 \mathrm{E} 20.10)$

IF (ABS(FACTOR-FACTDRD) OLT.FACTER*1.F-R) GC TO 200

ITERMAX $=10$

IFIITERC.GT.ITFRMAX) GO TC 22.5

IF(ITERC.GT.10) GO TO 225

GO TO E5

200 CUNTINLE

PRINT 35, ITFRC, FACTOR

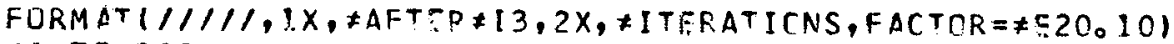
GU TC 999

225 PRINT 40, ITERC, FACTIR, FACTCPD

40

999 FURMAT $/ / / / /, 1 X, \neq A F T E R \neq, I 3,2 X, \neq I T E R A T I O N S, N C$ CONVERGENCF, $1 /, 1 X$, IFTHE LAST TWI FACTOR VALUES AREF, 2(5X,F20.10)) CUNTINUE

CALL $~ E X I T$

END

SUUROUTINE SUBSIN $(Z, N F T, S N, C S)$

DIMENSION SN(I), CSII)

$P 1=3.141592654$

DO $10 \quad I=1$, NFT

$S N(I)=S I N(2 . * P I * F \operatorname{LOAT}(I-1) * L)$

$\operatorname{CS}(1)=\operatorname{COS}(? . * P I * F \operatorname{PLAT}(I-1) * 2)$

IF(ABS(SN(I),$L T \cdot 1 . C T-8) \quad S N(I)=0$ 。

IF (ABS(CSII)) $0 L T \circ 100:-8) \quad C S(I)=0$ 。

10

CUNTINUE

RETURN

$\$$

END 
Program INTERI

This program calculates the integral I (equation 6.10) used in the Lévêque-like mass-transfer coefficient calculations. The input is identical to that of INTER. The subroutines BAND, MATINV and POLY are required. These are 1isted in INTER. 


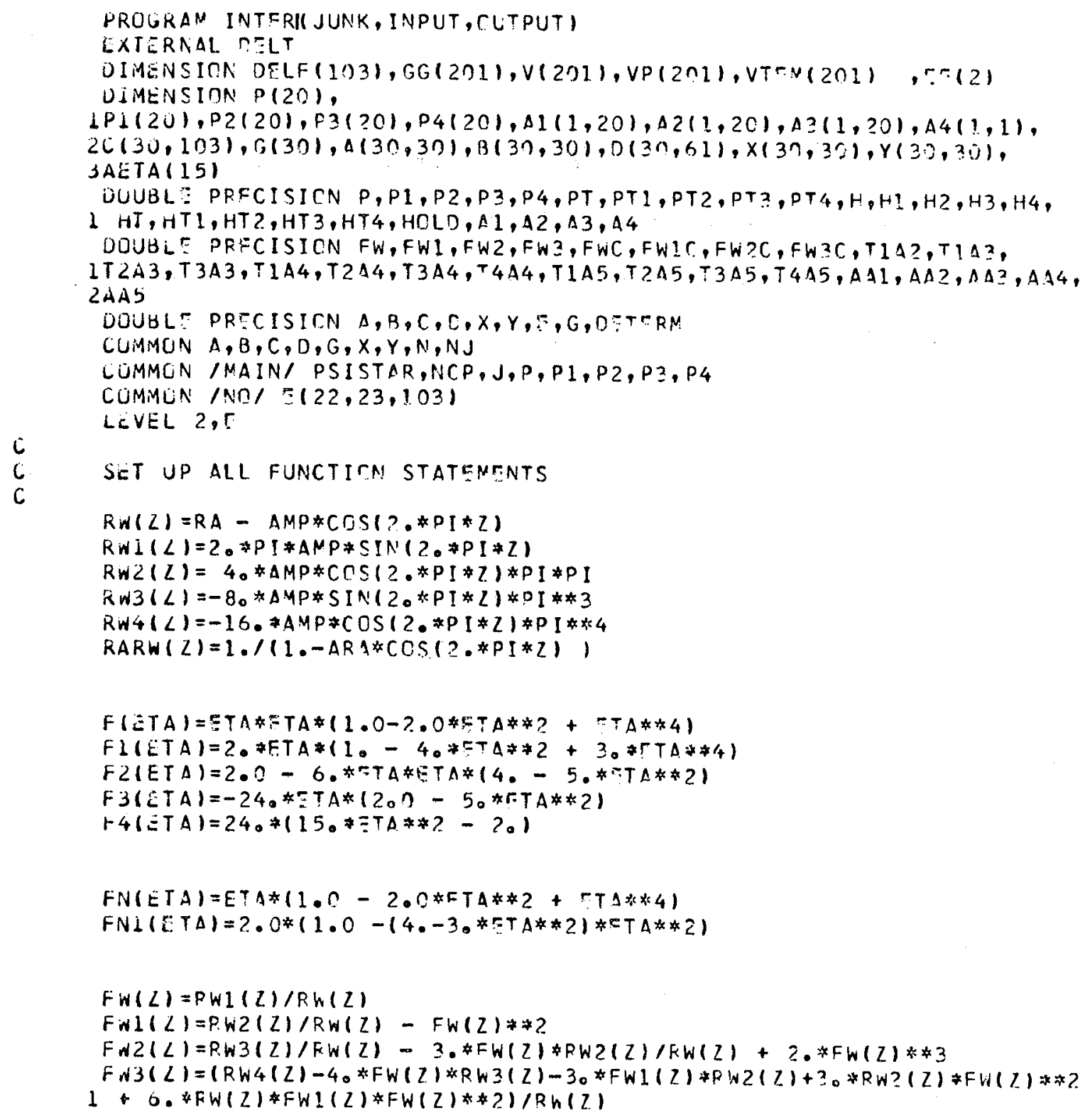

$H T(G T A, P T)=F(T+A) * P T$

HTI(ETA,PT,PTI ) =FI(RTA) $\# P T+F(F T A) * P T 1$

HT2 $(\therefore T A, P T, P T 1, P T 2)=F 2(F T A) * P T+20 * F 1(T T A) * P T 1+F(-T A) * P T 2$

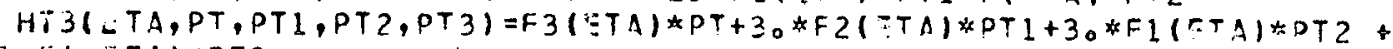
$1 F(\Xi T \Delta) \neq P T 3$

$H T+(E T A, P T, P T 1, P T 2, P T 3, P T 4)=F 4(C T A) * P T+4 . * F(F T A) * P T ?+6 . * F(F T$ (A)*PT $2+40 * F 1(E T A) * P T 3+F(C T A) * P T 4$

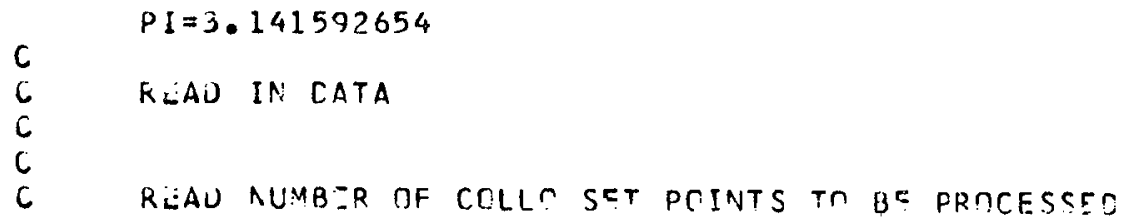


C

5. REAU 5, NCS

C... 900 III $=1 ., N C S$

C

C REAU IN CCLLO SET DATA

C

RIAU 6, NCP,NJ, NSKIP,ZMIA, ZNAX,RA, AMP

6 FUKMAT (3I5,4F15.1CI

RLAD $7,(A: T A(I), I=1, N C P)$

7 FURMAT (5FI5.10)

PRINT SO3,NCP,NJ,ZMAX,RA,ANP

903 FUKMAT(1HI, IX, $1 P U I N T S=*, I 5 / 1 X, \neq Z M A X=* F 15, J O / 1 X, \neq W A L L$ PADIUS $= \pm, F 15.1 \cap, 1 \cap X, * A M P=*$, $1 F 13.10 / 1 / 1$

PKINT 7, (A:TAII),I=I,NCP)

$A M A=A N P / P A$

C

\section{START EAND CALCULATIONS}

$D E L Z=(Z M A X-2 M I N) / F I C \Delta T(N J-3) \quad \& \quad N=2 * \Lambda C P \quad \$ \quad J=0$

DU $9 \quad I=1 ; N$

Di $9 K=1, N$

$Y(I, K)=0$.

$9 \quad x(1, K)=0$

$10 \quad J=J+1$

$\angle=U E L L * F I O A T(J-2)+Z M I N$

$F W C=F W(Z) \& F W 1 C=F W 1(Z) \$ F W 2 C=F W 2(Z) \$ F W 3 C=F n 3(Z)$

DO $11 I=1, N \quad G(I)=0 . \quad \$ \quad$ DE $11 K=1, A \quad \$ \quad(I, K)=0$. $B(I, K)=0$ 。

$11 \quad \mathrm{O}(I, K)=\mathrm{C}$ 。

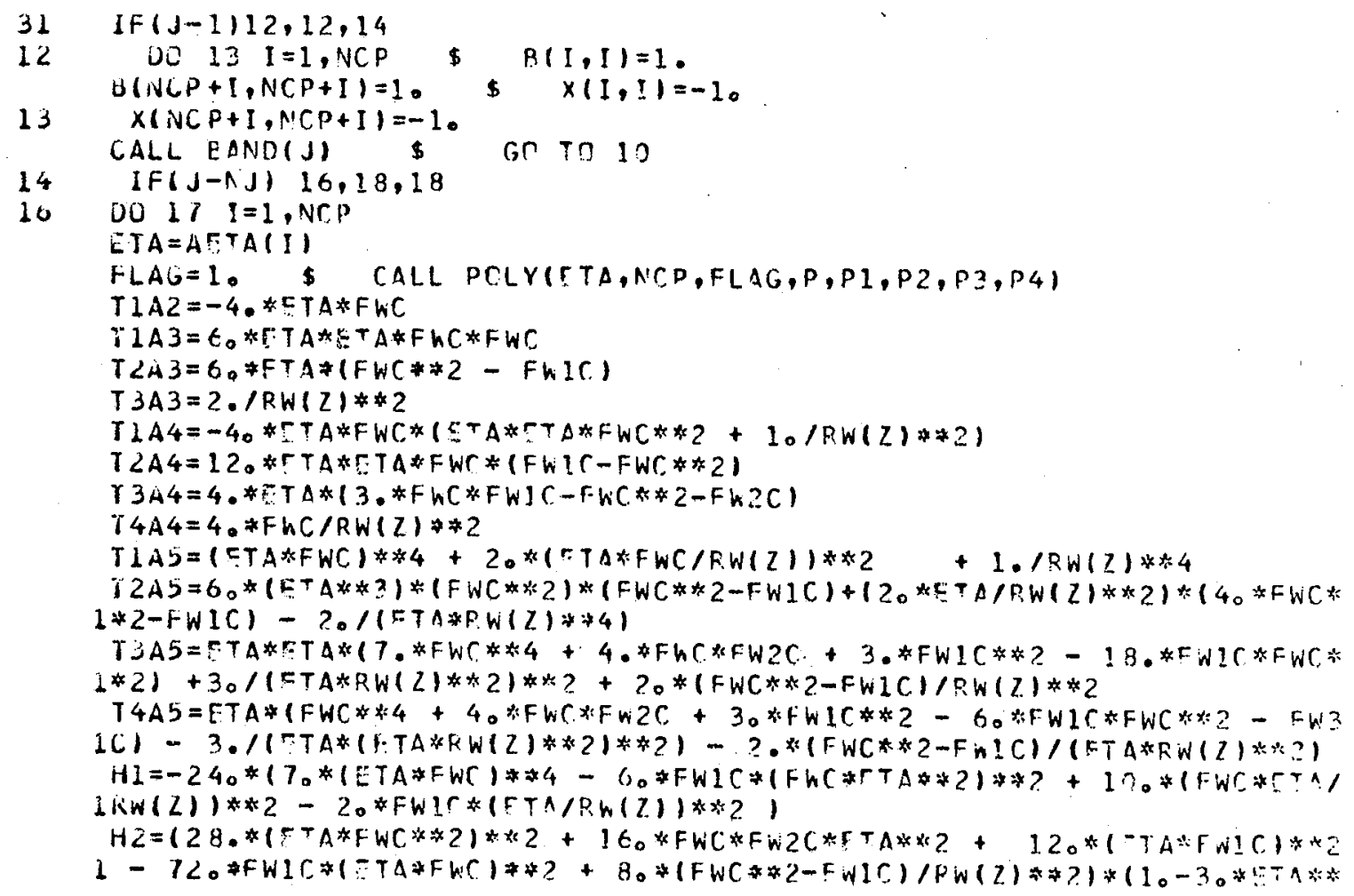




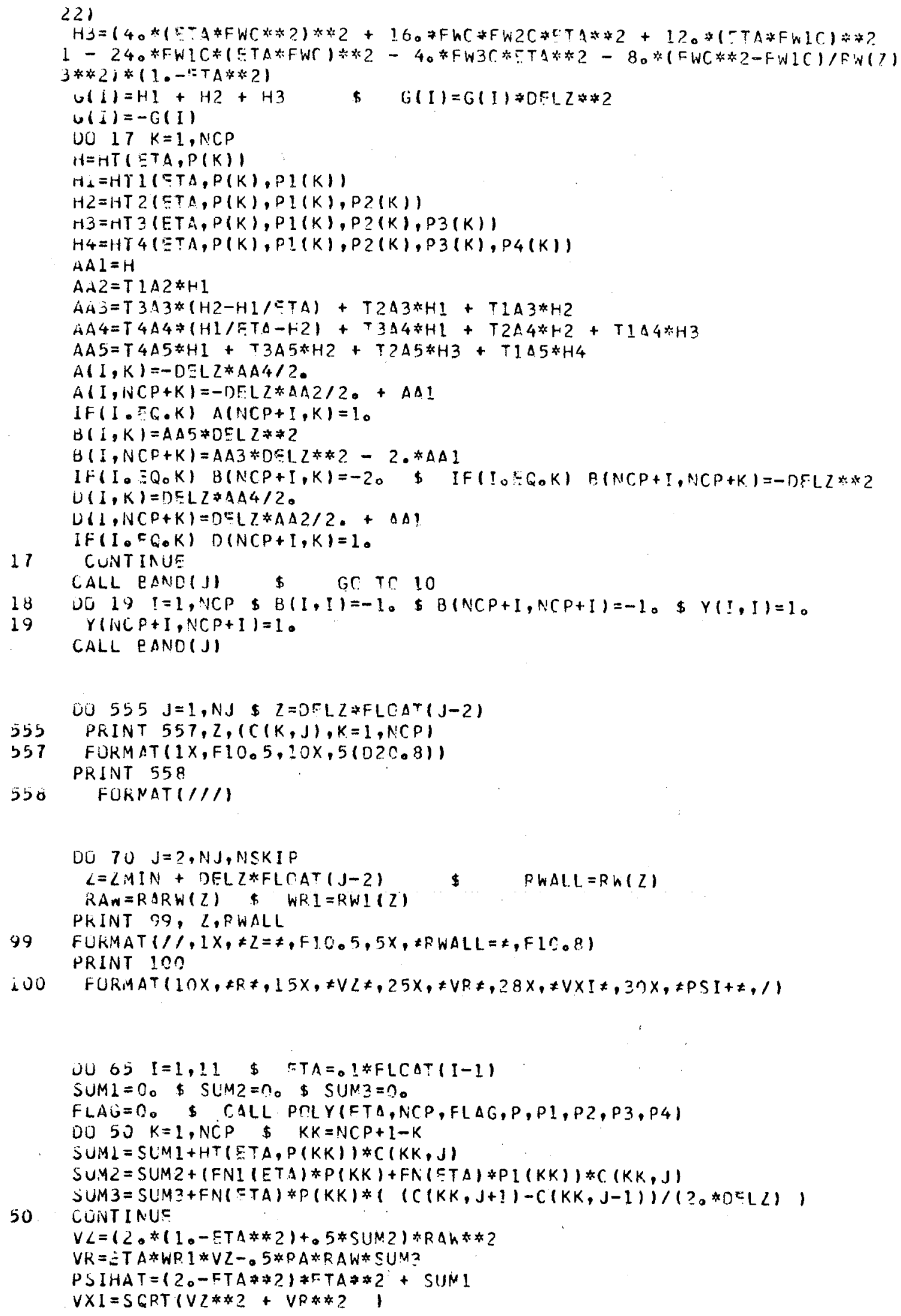




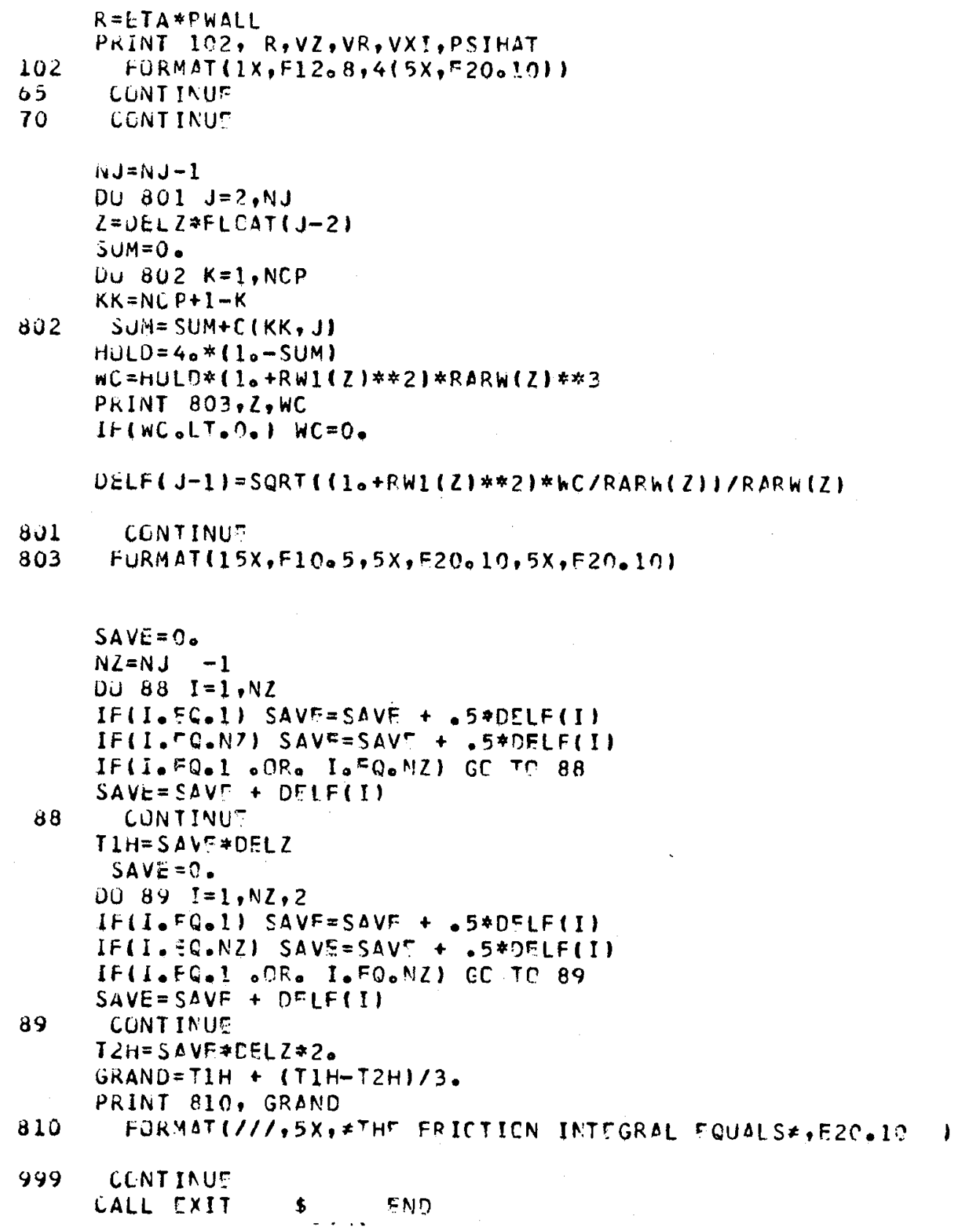


Program MAIN

This program calculates the quantity

$$
\left(2 \varepsilon / \operatorname{ar}_{A d}\right)
$$

assuming the sinusoidal PCT model. The input parameters are: $\mathrm{N}$, the number of parameter sets to be processed; RA and AMP, the average radius and wal1 amp1itude. 


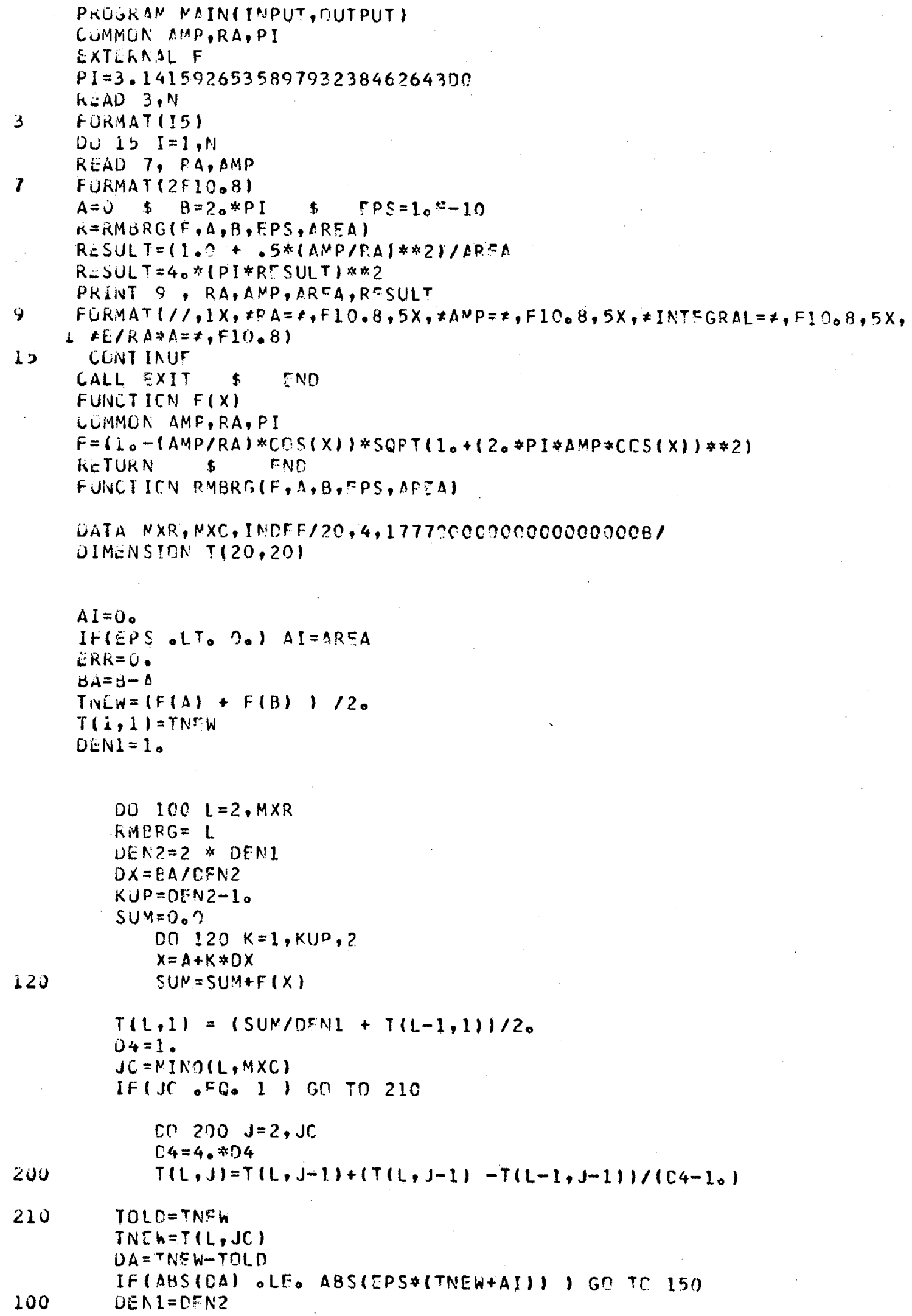




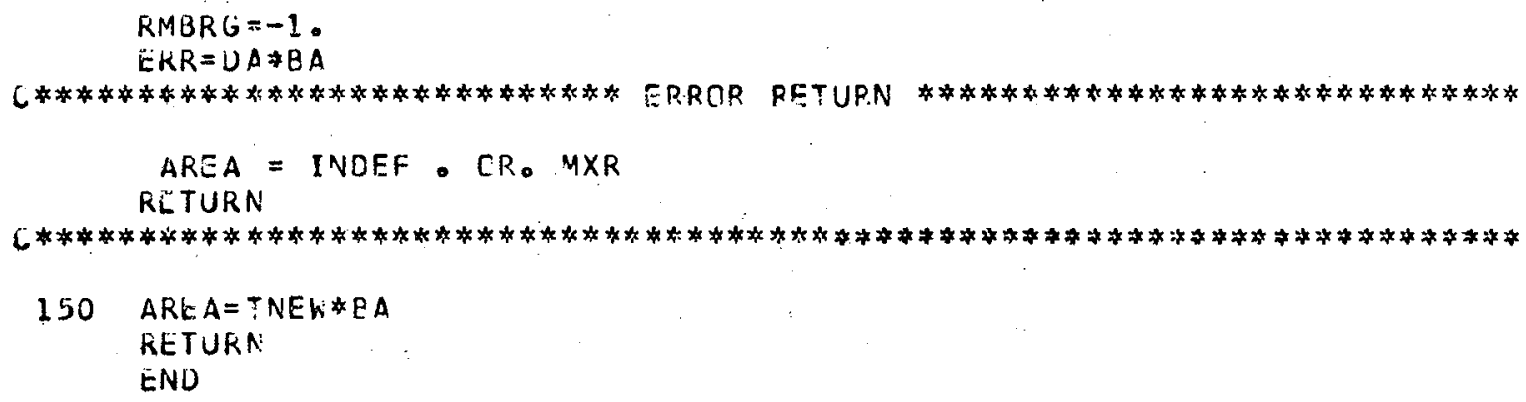


Program PHETHET

This program calculates the dimensionless ohmic potential

drop in a porous electrode according to the Newman and Tiedemann analysis. The Péclet number and conversion are the specified parameters. The Péclet numbers are specified in a DATA statement and the $\theta_{L}$ values axe read. The function FSHB is the Sherwood number $\left(\mathrm{Sh}_{B}\right)$ correlation and the function EDO is the dimensionless dispersion coefficient $\left(E / D_{o}\right)$ correlation. The film Sherwood number $\left(\varepsilon k_{f} / a D_{o}\right)$ is also calculated. 


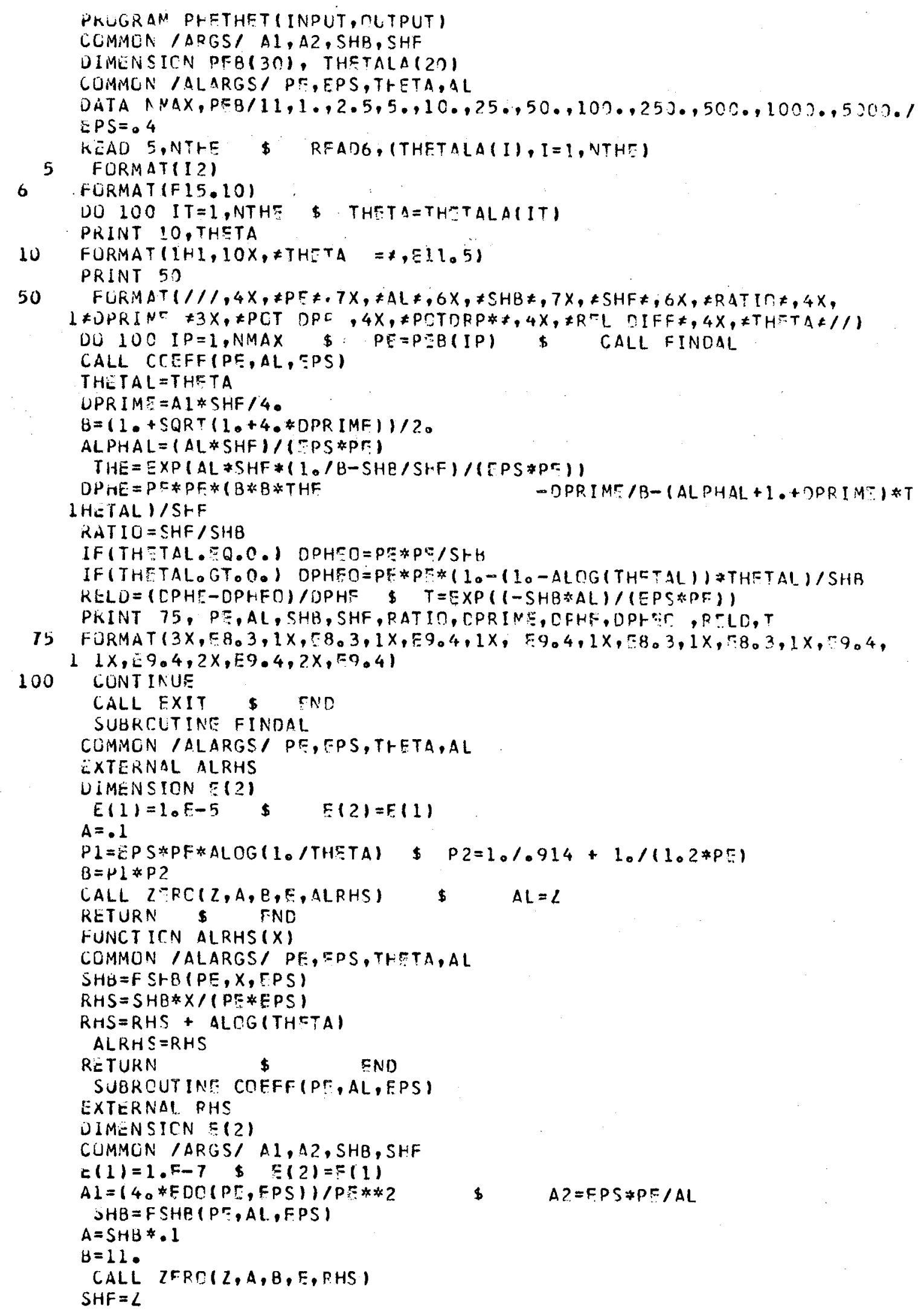




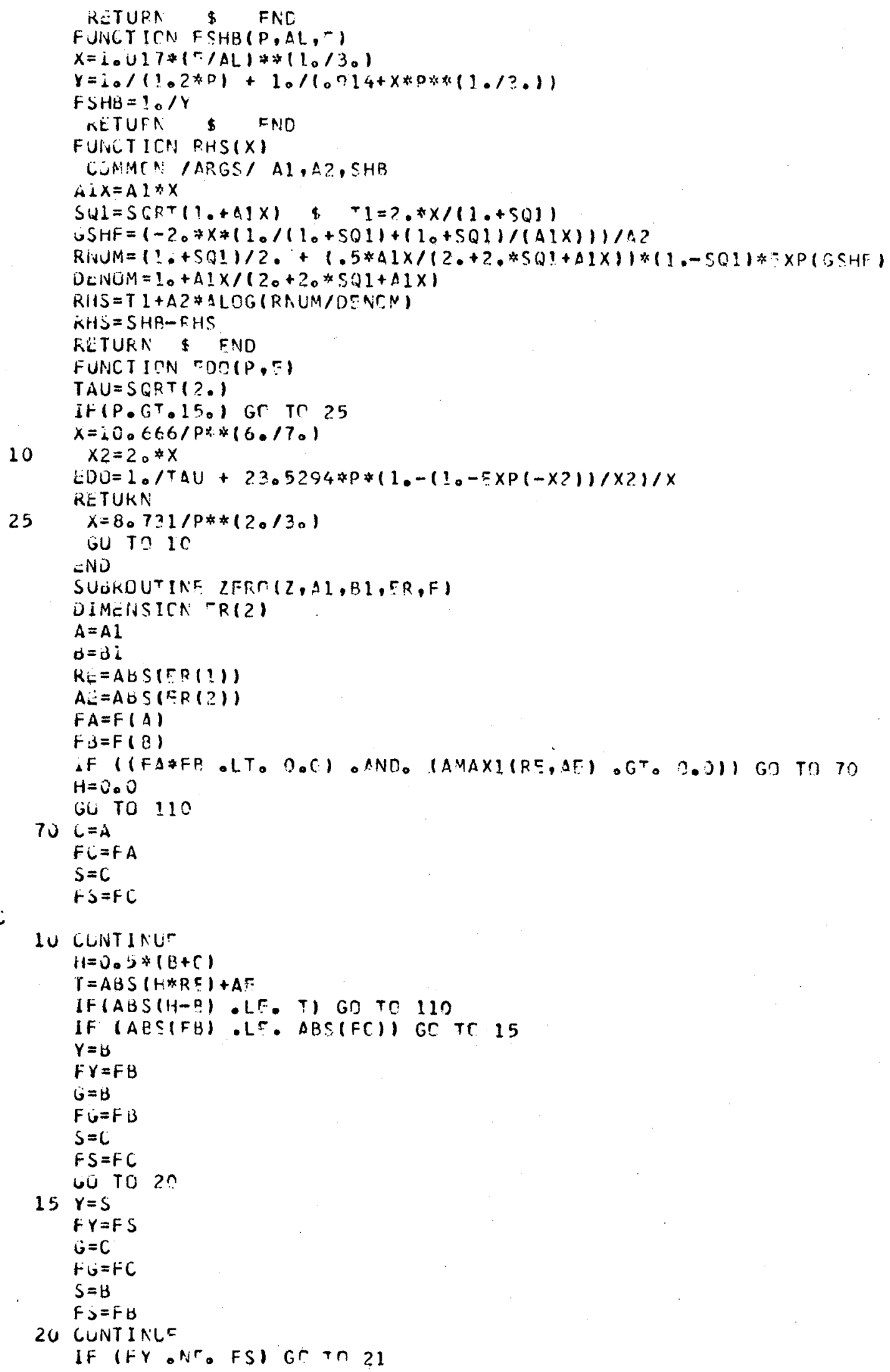




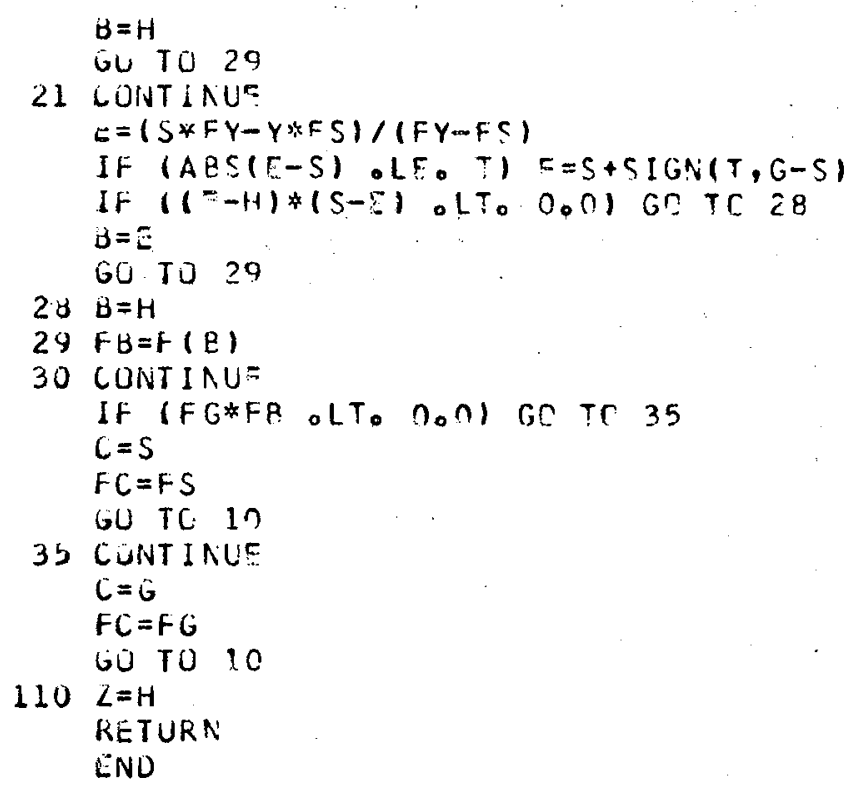


Program PHE

This routine is similar to PHETHET. The conversion and potential drop are calculated as functions of specified aL and $\mathrm{Pe}_{B}$. The film Sherwood number $\left(\varepsilon k_{f} / a D_{0}\right)$ is also calculated. 
1

PRUGRAM PFE(INPUT, DUTPUT)

ULMENS ION PEB $(30), A A L(10)$

CUHMON! /ARGS/ A1, 22 , SHB, SHF

UATA $P$ CB/.001,00025,0005,001,0C25,005,01,025,05,10,2.5,50,10,1

$1<b_{0}, 50,100,250,500,1000,5000,1$

NMAX $=2 C$

$E P S=.4$

5 FOKMATI12)

6 FURMATIFIO.5)

$R \triangle A D$ 5,NAL \$ RCAD 6, (AAL(I),I I, MAL)

DO 100 IA $=1, N A L \$ A L=A A C(I A) \$ P R I N T 10, A L$

10

FORM $\triangle T(1 H 1,10 X, * * * * \quad A L=\neq, F 1), 5, \neq * * * \neq 1 /)$

PRINT 50

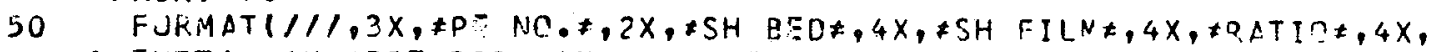

$I \neq T H E T A \neq, 4 X, \neq P Q T$ DRP $\neq, 4 X, \neq D P P, I M E *, 4 X, \neq P O T D R P * \neq 11$

DO $1001 P=1, N M A X$

$\mathrm{PL}=\mathrm{PEE}$ (IP)

LALL CCEFF(PF, AL, EPS)

THETAL $=$ PP(1-AL*SHB) / $($ PPS *PP $)$

$D P R I M E=A 1 * S H F / 40$

$B=(1 \bullet+S Q R T(1 \bullet+4 \bullet * D P R I M P I) / 2$.

$A L P H A L=(A L * S H F) /(P P S * P=)$

$T H E=F \times P(A L * S H F *(10 / B-S H E / S H F) /(E P S * P C))$

$D P H E=P E * P F *(B * B * T H F \quad-D P R I M T / P-(A L P H A L+1+D P R I M T) * T$

1 HETAL ) /SHF

KATIO $=S H F / S H B$

IF(TH TAL.EQ.0.) DPHER=PE*PE/SHB

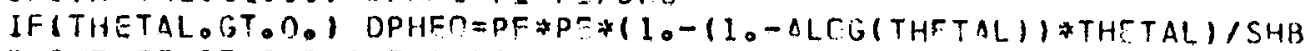

PRINT 75, PE, SHB, SHF, RATIC, THET AL, DPHF, CPRIME, DFHFO

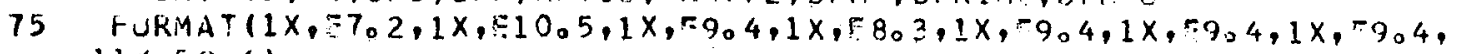

$11 \times, E 9.41$

100 CONTINUE

CALL EXIT \$ TND

SUIRCLTINS COEFFIPE,AL, SPS)

EXTERNAL RHS

UIMENSION $E(2)$

COMMON /ARGS/ A1, $\triangle 2, S H B, S H F$

$E(1)=1, E-7 \quad \& \quad P(2)=E(1)$

$A 1=14 . * C D O(P F, E P S) / / P F * * 2$

$S H B=F S H B(P C, A L, E P S)$

$\$ \quad A 2=E P S * P S / A L$

$A=S+1 B * 1$

$B=11$.

CALL ZERO (Z,A,B, E,PHS)

$S H F=L$

RETURA \$ END

FUNCTION FSHB(P, AL,F)

$X=1.017 *(\Gamma / A L) * *(1 . / 30)$

$Y=1.1(1.2 * 0)+1.1(.914+X * P *(1.13 .1)$

$F S H B=10 / Y$

RETURA \$ END

FUNCTICN RHS $(X)$

LUMMCN /ARSS/ AI,AZ, SHB

$A$ I $X=A I * X$

$S Q I=S Q R T\left(1_{0}+A 1 X\right) \& T 1=2_{0} * x /\left(1_{0}+S Q 1\right)$

$G S H F=(-20 * X *(10 /(10+S Q 1)+(10+501) /(A) x) 1) / A 2$

KIVUM $=(1 .+S(1)) / 2++1.5 * A 1 X /(2,+2 . * S Q 1+A 1 X)) *(1 .-S Q 1) * X P(G S H F)$

$\checkmark E N O M=1 \bullet+A 1 X /(2, \bullet+20 * S Q 1+\Delta \mid X)$

$R H S=T 1+A 2 * A L O G(R N U M / D E N O M)$

RHS $=S H E-R H S$

RETURN \& END 


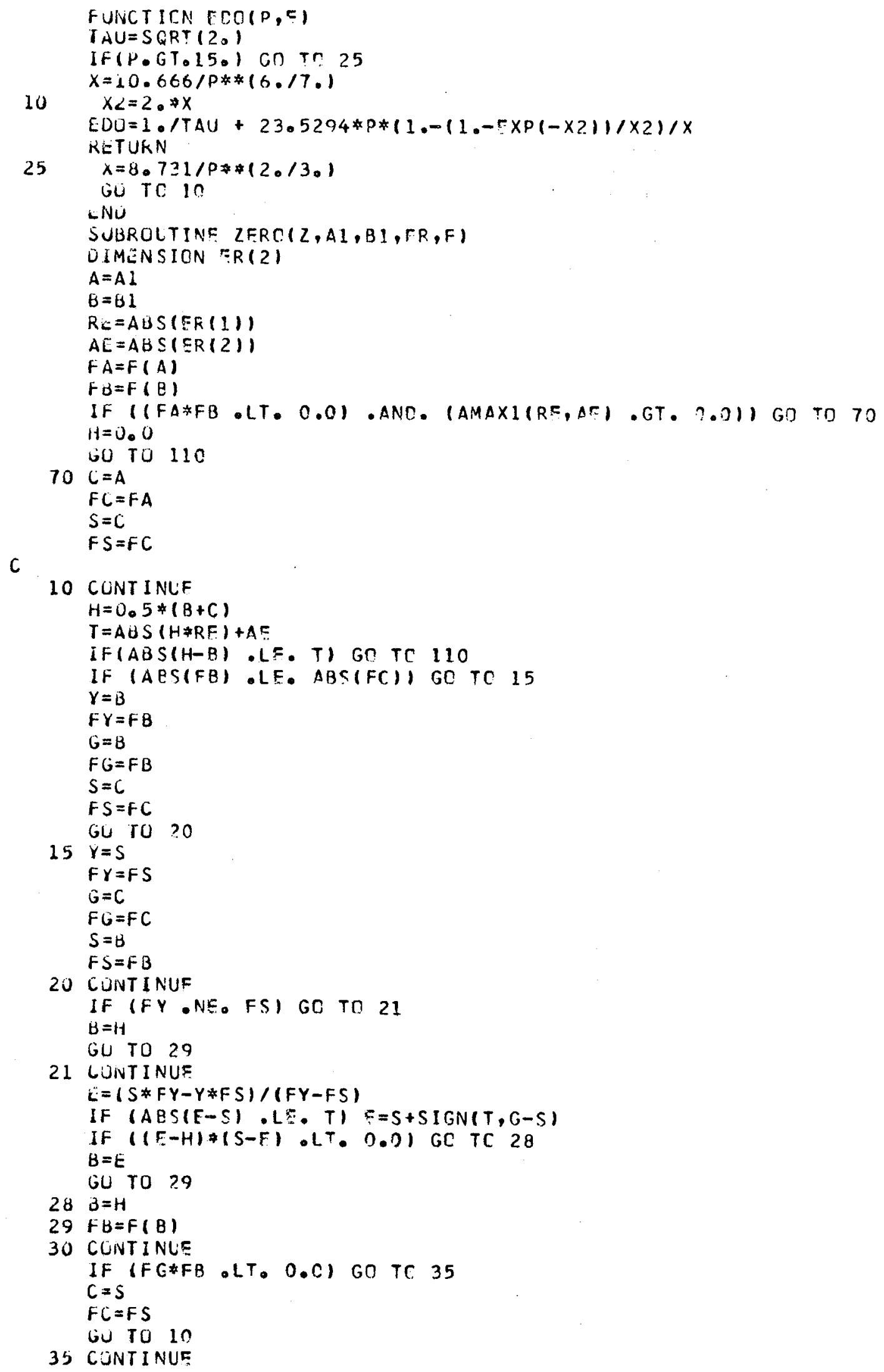




$$
\begin{aligned}
& C=i \\
& F C=F G \\
& \text { GL TL } 10 \\
& 110 \mathrm{~L}=\mathrm{H} \\
& \text { KETURN } \\
& \text { ENU }
\end{aligned}
$$




\section{Appendix $\mathrm{C}^{*}$ \\ Solution of Coupled, Ordinary Differential Equations with Associated Constant Unknowns}

The BAND subroutine is designed to effect efficient solution of coupled difference equations (Newman 108,63). At an interior point $j$, these equations take the form

$$
\sum_{k=1}^{N}\left[A_{i, k}(j) C_{k}(j-1)+B_{i, k}(j) C_{k}(j)+D_{i, k}(j) C_{k}(j+1)\right]=G_{i}(j)
$$

Frequent1y, there will be unknowns which are independent of position. An example is the eigenvalue in an eigenvalue problem (see Newman 64, pp. 204-208). An example encountered in this thesis involves collocation solutions of problems with periodic boundary conditions. Here there may be one or two constant unknowns for each spatially variable unknown. Such situations can be handled with the standard BAND subroutine by appending to NN spatially dependent unknowns $C_{k}(j)$ a set of unknown constants $C_{k}$ decomposed in turn into two subsets. For the first subset, for $1=\mathrm{NN}+1, \ldots, \mathrm{N} 2$, one writes for equation 1

$$
C_{i}(j)=C_{i}(j+1), \quad j \neq N J,
$$

and for the second subset, for $i=N 2+1, \ldots, N$, one writes

$$
c_{i}(j)=c_{i}(j-1), j \neq 1 \text {. }
$$

\footnotetext{
* Written by J. Newman.
} 
In both cases, no value is explicitly specified for the constant unknown. Instead, the form of equation 2 , excluding the point at the right for $j=N J$, permits the specification of an additional boundary condition or auxiliary condition on the spatially dependent unknowns. Similarly, the form of equation 3 permits an extra boundary condition at the left, at $j:=1$.

Use of the standard BAND subroutine and equations 2 and 3 may be attractive when the unknown constants are few in number, but significant improvements in computational efficiency result when the special subroutine in this appendix is used for problems with more unknown constants. This improvement in efficiency is achieved by avoiding calculations of a trivial nature and avoiding the storage of numbers of a trivial nature. For example, the standard solution form assumed for equation 1 is

$$
c_{i}(j)=\xi_{i}(j)+\sum_{l=1}^{N} E_{i, \ell}(j) C_{\ell}(j+1) .
$$

Substitution into equation 2 would yield, after trivial calculations, (for $i=\mathrm{NN}+1, \ldots, \mathrm{N} 2)$

$$
\xi_{i}(j)=0 \quad \text { and } \quad E_{1, \ell}(j)=\delta_{1, l}
$$

where $\delta_{1, \ell}$ is the Kronecker delta equal to 1 if $1=\ell$ and equal to zero otherwise. 


\section{Interior points}

For an interior point $j$, the assumed solution form for a spatially dependent variable is

$$
C_{1}(j)=\xi_{i}(j)+\sum_{\ell=1}^{N N} E_{i, \ell}(j) C_{\ell}(j+1)+\sum_{\ell=N N+1}^{N 2} E_{i, \ell}{ }^{(j) C_{\ell},(C-6)}
$$

a simplification of equation 4. A consideration of the disposition of the boundary conditions suggests that the unknown constants $C_{i}$ for $i=N 2+1, \ldots, N$ need not appear in equation 6 and can themselves be expressed at any point $j$ in the assumed solution form

$$
c_{i}=\xi_{i}(j)+\sum_{\ell=1}^{N N} E_{i, \ell}(j) C_{\ell}(j+1)+\sum_{\ell=N N+1}^{N 2} E_{i, \ell}(j) C_{\ell} \cdot(C-7)
$$

(New arrays can be defined for $E E$ because it is not necessary to store the values for more than one value of $j$. )

Consider first an interior point $j$ for $j>2$, and assume that values of the $\xi, E$, and $E E$ arrays have been obtained for $j-1$. Substitution of equations 6 and 7 into equation 1 (for $i=1,2, \ldots, N N$ ) yields equations to solve for $\xi_{i}(j)$ and $E_{i, \ell}(j)$ :

$$
\sum_{k=1}^{N N} b_{i, k}(j) \xi_{k}(j)=g_{i}(j)
$$

and

$$
\sum_{k=1}^{N N} b_{i, k}(j) E_{k, \ell}(j)=-d_{i, \ell}{ }^{(j)} \text { for } \ell=1,2, \ldots, N 2 \text {, }
$$


where the square $\mathrm{NN}$ by $\mathrm{NN}$ matrix of coefficients is given by

$$
\begin{gathered}
b_{i, k}(j)=B_{i, k}(j)+\sum_{\ell=1}^{N N} A_{i, \ell}(j) E_{\ell, k}(j-1) \\
+\sum_{\ell=N 2+1}^{N}\left[A_{i, \ell}(j)+B_{i, \ell}(j)+D_{i, \ell}(j)\right] E_{\ell, k}(j-1) .
\end{gathered}
$$

The right side of equation 8 is

$$
\begin{gathered}
g_{i}(j)=G_{i}(j)-\sum_{\ell=1}^{N N} A_{i, \ell}(j) \xi_{\ell}(j-1) \\
-\sum_{\ell=N 2+1}^{N}\left[A_{i, \ell}(j)+B_{i, \ell}(j)+D_{i, \ell}(j)\right] \xi_{\ell}(j-1) .
\end{gathered}
$$

The right side of equation 9 is simply given by

$$
d_{i, \ell}(j)=D_{i, \ell}(j) \text { for } \ell=1,2, \ldots, \text { NN }
$$

but takes the form

$$
\begin{aligned}
d_{i, \ell}(j) & =A_{i, \ell}(j)+B_{i, \ell}(j)+D_{i, \ell}(j)+\sum_{k=1}^{N N} A_{i, k}(j) E_{k, \ell}(j-1) \\
& +\sum_{k=N 2+1}^{N}\left[A_{i, k}(j)+B_{i, k}(j)+D_{i, k}(j)\right] E_{k, \ell}(j-1)
\end{aligned}
$$

for $\ell=N N+1, \ldots, N 2$. Bear in mind that $i$ takes on values from 1 through NN in equations 8 through 13.

To obtain values of $\xi_{i}(j)$ and $E_{i, \ell}(j)$, substitute equations 6 and 7 into equation 3 (for $i=N 2+1, \ldots, N$ ), yielding 


$$
\begin{array}{r}
\xi_{i}(j)=\xi_{i}(j-1)+\sum_{k=1}^{N N} E_{i, k}(j-1) \xi_{k}(j), \quad(C-14) \\
E_{i, \ell}(j)=\sum_{k=1}^{N N} E_{i, k}(j-1) E_{k, \ell}(j) \text { for } \ell=1,2, \ldots, N N,(C-15)
\end{array}
$$

and

$$
E_{i, \ell}(j)=E E_{i, \ell}(j-1)+\sum_{k=1}^{N N} E_{i, k}(j-1) E_{k, \ell}(j)
$$

for $\ell=\mathrm{NN}+1, \ldots, \mathrm{N} 2$.

$\underline{\text { Right }}$ boundary

For $j=N J$, the equations are simpler because terms in $D_{i, k}(j)$ are absent. However, the problem statement is generalized by including terms at $j-2$, so that complex boundary conditions (involving derivatives) can be treated. At $j=\mathrm{NJ}$, equation 1 is replaced by equations of the form

$$
\sum_{k=1}^{N}\left[Y_{i, k} C_{k}(j-2)+A_{i, k}(j) C_{k}(j-1)+B_{i, k}(j) C_{k}(j)\right]=G_{i}(j) \cdot(C-17)
$$

This equation applies for $i=1,2, \ldots, N 2$, while equation 3 still applies for $1=\mathrm{N} 2+1, \ldots, \mathrm{N}$.

First use equation 6 to eliminate $c_{k}(j-2)$. This leads us to define auxiliary quantities as follows:

$$
A_{i, k}^{\prime}=A_{i, k}(j)+\sum_{\ell=1}^{N N} Y_{i, \ell^{E}, k}(j-2)
$$


for $k=1,2, \ldots, N 2$, and

$$
G_{i}^{\prime}=G_{i}(j)-\sum_{\ell=1}^{N N} Y_{i, \ell} \xi_{\ell}(j-2)
$$

Next use equation 6 to eliminate $c_{k}(j-1)$ and equation 7 to eliminate $C_{k}$ for $k=N 2+1, \ldots, N$. The equations to solve then become

$$
\sum_{k=1}^{N 2} b_{i, k}(j) c_{k}(j)=g_{i}
$$

where the square $\mathrm{N} 2$ by $\mathrm{N} 2$ matrix of coefficients has the form

$$
\begin{gathered}
b_{i, k}(j)=B_{i, k}(j)+\sum_{\ell=1}^{N N} A_{i, \ell}^{\prime} E_{\ell, k}(j-1) \\
+\sum_{\ell=N 2+1}^{N}\left[Y_{i, \ell}+A_{i, \ell}(j)+B_{i, \ell}(j)\right] E E_{\ell, k}(j-1)
\end{gathered}
$$

for $k=1,2, \ldots, N N$. The same form applies for $k=N N+1, \ldots, N 2$, but $Y_{i, k}$ and $A_{i, k}^{\prime}$ are also added into $b_{i, k}(j)$. The right side of equation 20 has the form

$$
g_{i}=G_{i}^{\prime}-\sum_{\ell=1}^{N N} A_{i, \ell}^{\prime} \xi_{\ell}(j-1)-\sum_{\ell=N 2+1}^{N}\left[Y_{i, \ell}+A_{i, \ell}(j)+B_{i, \ell}(j)\right] \xi_{\ell}(j-1)
$$

The inversion of equation 20 then gives values of $C_{k}(j)$ at $j=N J$ as we11 as the constants $C_{k}$ for $k=N N+1, \ldots, N 2$. The constants $C_{k}$ for $k=N 2+1, \ldots, N$ can now be obtained by applying equation 7 with $j=N J-1$. Back substitution with equation 6 for decreasing values of $j$ then completes the solution for $c_{k}(j)$. 


\section{Left boundary}

The governing equations at $j=1$ take the form

$$
\sum_{k=1}^{N}\left[B_{i, k}(j) C_{k}(j)+D_{i, k}(j) C_{k}(j+1)+x_{i, k} C_{k}(j+2)\right]=G_{i}(j),(C-23)
$$

except that the simple form 2 still applies for $i=N N+1, \ldots, N 2$. The $D_{i, k}(j)$ term is missing from equation 23, but the $x_{i, k}$ term is added in order to permit the use of an image point and derivative boundary conditions. The solution form 6 is extended at $j=1$ to read

$$
c_{i}(j)=\xi_{i}(j)+\sum_{\ell=1}^{N 2} E_{i, \ell}(j) c_{\ell}(j+1)+\sum_{\ell=1}^{N N} x_{i, \ell} c_{\ell}(j+2) .
$$

Equation 7 must be extended to a form similar to equation 24 when applied to the constants $C_{i}$ for $i=N 2+1, \ldots, N$. Substitution of equation 24 and the extended form of equation 7 into equation 23 leads to matrix equations which should be solved for $\xi_{k}(1), E_{k, \ell}(1)$ (for $\left.\ell=1, \ldots, N 2\right)$, and $x_{k, l}$ (for $\ell=1, \ldots, N N$ ), all for $k=1, \ldots, N N$ and for $k=N 2+1$, $\ldots, N$. In the computer program, the matrix can be collapsed somewhat since $k$ values from $N N+1$ through $N 2$ are absent and equation 23 is not applied for $i=N N+1, \ldots, N 2$.

The point at $j=2$ cannot be treated completely like an interior point because equation 24 should be used instead of equation 6 to eliminate $C_{k}(j-1)$ from equation 1 .

\section{Program notes}

The subroutine BAND $(J)$ 1isted in this appendix can be used in the programs of chapter 5 just like the regular BAND subroutine listed 
in appendix $B$. This means in particular that $N N$ and $N 2$ are calculated within the subroutine in a special manner, whereas in general they would be used as parameters for the subroutine.

Some slight additional economy could be achieved by reducing the size of the $\mathrm{EE}$ array, since the first index does not range over values from 1 through $\mathrm{N} 2$. The programming would then be somewhat more complex. Another economy measure would require that only the $B$ array, not $A, D, X$, or $Y$ be used in equations 1,17 , and 23 when referring to one of the constant unknowns in the calling program. This could then be assumed in the subroutine. 


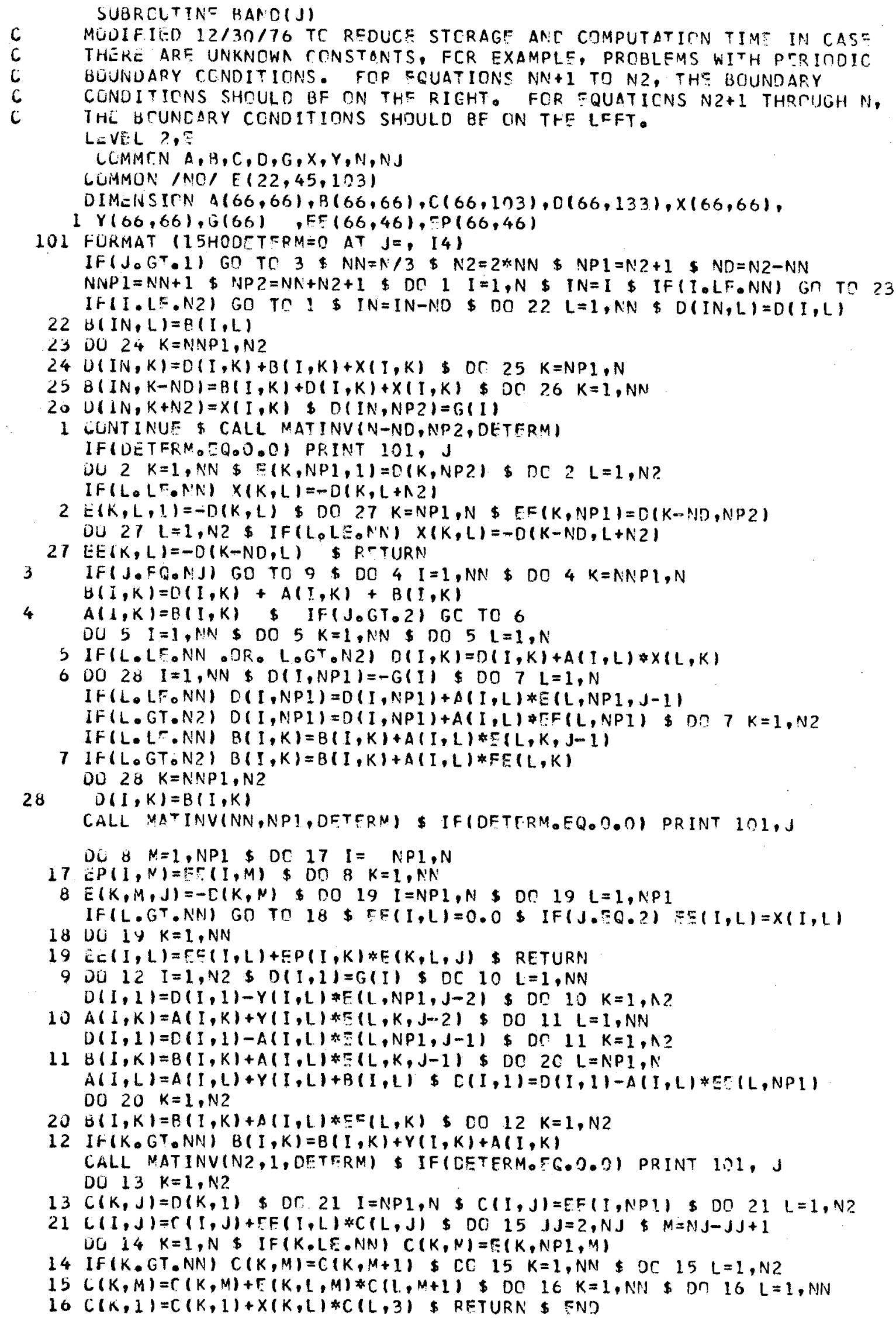




\section{Appendix D}

Data Compilation

The following table lists the measured values for all the pertinent variables of the mass-transfer experiments. All experiments were carried out at $25^{\circ} \mathrm{C}$. The notation is the same as that used in the main body of the thesis. Some preliminary data reduction has been performed and is reported here. The diameter of the bed was 3.00" and the packing was $1 / 8^{\prime \prime}$ spherical bearings for all experiments. Runs 1 through 25 inclusive were made in the power supply contro1 mode while the remainder were done with potentiostatic contro1. 


\begin{tabular}{|c|c|c|c|c|c|c|c|c|c|}
\hline Run & $\varepsilon$ & $\mathrm{aL}$ & $\begin{array}{c}\mathrm{q} \\
(\mathrm{m} \ell / \min )\end{array}$ & $\begin{array}{c}{\left[\mathrm{Cu}^{++}\right]_{F}} \\
(\underline{M})\end{array}$ & $\begin{array}{c}{\left[\mathrm{H}_{2} \mathrm{SO}_{4}\right]_{\mathrm{F}}} \\
\underline{\mathrm{M}}\end{array}$ & $\begin{array}{c}\text { Sample } \\
\# \#\end{array}$ & $\begin{array}{l}\mathrm{V}_{\mathrm{c}}-\phi_{\mathrm{R}} \\
(\mathrm{mV})\end{array}$ & $\begin{array}{c}I_{c} \\
(m A)\end{array}$ & $c_{L} / c_{F}$ \\
\hline 10 & 0.366 & $\begin{array}{r}30.1 \\
\pm .7\end{array}$ & $\begin{array}{c}18.4 \pm \\
0.1\end{array}$ & $1.10 \times 10^{-3}$ & 0.9704 & $\begin{array}{l}1 \\
2 \\
3 \\
4 \\
5 \\
6 \\
7 \\
8\end{array}$ & $\begin{array}{l}-45 \\
-78 \\
-100 \\
-138 \\
-187 \\
-234 \\
-294 \\
-307\end{array}$ & $\begin{array}{l}28.0 \\
40.2 \\
42.4 \\
44.0 \\
45.2 \\
45.9 \\
47.6 \\
51.6\end{array}$ & $\begin{array}{l}0.600 \\
0.407 \\
0.374 \\
0.337 \\
0.315 \\
0.307 \\
0.278 \\
0.292\end{array}$ \\
\hline 11 & 0.391 & $\begin{array}{c}29.0 \pm \\
1.0\end{array}$ & $\begin{array}{c}35.5 \pm \\
0.1\end{array}$ & $\begin{array}{l}1.09 \pm .03 \\
\times 10^{-3}\end{array}$ & 0.9704 & $\begin{array}{l}1 \\
2 \\
3 \\
4 \\
5 \\
6 \\
7 \\
8 \\
9 \\
10\end{array}$ & $\begin{array}{l}-43 \\
-65 \\
-90 \\
-106 \\
-130 \\
-165 \\
-197 \\
-230 \\
-300 \\
-330\end{array}$ & $\begin{array}{l}27.1 \\
45.8 \\
54.6 \\
58.1 \\
61.3 \\
65.0 \\
67.3 \\
68.7 \\
71.6 \\
74.5\end{array}$ & $\begin{array}{l}0.815 \\
0.654 \\
0.575 \\
0.552 \\
0.519 \\
0.484 \\
0.469 \\
0.459 \\
0.443 \\
0.443\end{array}$ \\
\hline 12 & 0.382 & $\begin{array}{c}30.4 \pm \\
1.1\end{array}$ & $18.2 \pm 0.1$ & $\begin{array}{l}5.06 \pm .02 \\
\times 10^{-3}\end{array}$ & 0.9880 & $\begin{array}{l}1 \\
2 \\
3 \\
4 \\
5 \\
6 \\
7 \\
8\end{array}$ & $\begin{array}{l}-39 \\
-95 \\
-143 \\
-180 \\
-240 \\
-293 \\
-310 \\
-340\end{array}$ & $\begin{array}{l}160.0 \\
196.7 \\
208.3 \\
211.4 \\
213.7 \\
215.9 \\
218.2 \\
221.8\end{array}$ & $\begin{array}{l}0.480 \\
0.346 \\
0.306 \\
0.296 \\
0.292 \\
0.291 \\
0.287 \\
0.295\end{array}$ \\
\hline 15 & 0.372 & $100 \pm .2$ & $18.4 \pm 0.1$ & $\begin{array}{l}5.11 \times 10^{-3} \\
\pm .02\end{array}$ & 0.9880 & $\begin{array}{l}1 \\
2 \\
3 \\
4 \\
5 \\
6 \\
7 \\
8 \\
9 \\
10\end{array}$ & $\begin{array}{l}-57 \\
-79 \\
-93 \\
-102 \\
-119 \\
-136 \\
-156 \\
-228 \\
-272 \\
-290\end{array}$ & $\begin{array}{l}260.9 \\
276.4 \\
280.0 \\
281.9 \\
284.7 \\
286.9 \\
288.6 \\
290.0 \\
291.0 \\
293.0\end{array}$ & $\begin{array}{l}.0628 \\
.0511 \\
.0616 \\
.0532 \\
.0354 \\
.0354 \\
.0297 \\
.0227 \\
.0211 \\
.0204\end{array}$ \\
\hline
\end{tabular}




\begin{tabular}{|c|c|c|c|c|c|c|c|c|c|}
\hline Run & $\varepsilon$ & $\mathrm{aL}$ & $\begin{array}{c}\mathrm{q} \\
(\mathrm{m} \ell / \mathrm{min})\end{array}$ & $\begin{array}{c}{\left[\mathrm{Cu}^{++}\right]_{F}} \\
(\underline{M})\end{array}$ & $\begin{array}{c}{\left[\mathrm{H}_{2} \mathrm{SO}_{4}\right]_{\mathrm{F}}} \\
\underline{\mathrm{M}}\end{array}$ & $\begin{array}{c}\text { Sample } \\
\text { 非 }\end{array}$ & $\underset{(\mathrm{mV})}{\mathrm{V}_{\mathrm{c}}-\phi_{\mathrm{R}}}$ & $\begin{array}{c}I_{c} \\
(\mathrm{~mA})\end{array}$ & $c_{L} / c_{F}$ \\
\hline 18 & 0.385 & $\begin{array}{c}30.4 \pm \\
0.7\end{array}$ & $\begin{array}{c}35.6 \pm \\
0.2\end{array}$ & $4.89 \times 10^{-4}$ & 0.9408 & $\begin{array}{l}1 \\
2 \\
3 \\
4 \\
5 \\
6 \\
7 \\
8\end{array}$ & $\begin{array}{l}-53 \\
-74 \\
-100 \\
-121 \\
-144 \\
-173 \\
-286 \\
-298\end{array}$ & $\begin{array}{l}18.9 \\
26.7 \\
29.8 \\
31.3 \\
32.9 \\
33.8 \\
29.2 \\
35.0\end{array}$ & $\begin{array}{l}0.708 \\
0.577 \\
0.503 \\
0.470 \\
0.448 \\
0.421 \\
0.405 \\
0.411\end{array}$ \\
\hline 19 & 0.374 & $\begin{array}{c}30.3 \pm \\
0.7\end{array}$ & $\begin{array}{c}35.6 \pm \\
0.0\end{array}$ & $4.91 \times 10^{-4}$ & 0.9408 & $\begin{array}{l}1 \\
2 \\
3 \\
4 \\
5 \\
6 \\
7 \\
8\end{array}$ & $\begin{array}{l}-77 \\
-150 \\
-175 \\
-200 \\
-220 \\
-233 \\
-267 \\
-277\end{array}$ & $\begin{array}{l}207.5 \\
271.8 \\
291.1 \\
300.9 \\
310.4 \\
313.7 \\
319.9 \\
321.9\end{array}$ & $\begin{array}{l}0.613 \\
0.501 \\
0.464 \\
0.448 \\
0.432 \\
0.420 \\
0.418 \\
0.418\end{array}$ \\
\hline 20 & 0.385 & $100 \pm 2$ & $\begin{array}{c}35.6 \pm \\
0.2\end{array}$ & $5.04 \times 10^{-3}$ & 0.9408 & $\begin{array}{l}1 \\
2 \\
3 \\
4\end{array}$ & $\begin{array}{l}-48 \\
-119 \\
-175 \\
-194\end{array}$ & $\begin{array}{l}432.8 \\
526.2 \\
544.5 \\
551.0\end{array}$ & $\begin{array}{l}0.236 \\
0.0913 \\
0.0663 \\
0.0628\end{array}$ \\
\hline 21 & 0.406 & $\begin{array}{c}30.3 \pm \\
0.9\end{array}$ & $\begin{array}{l}7.60 \pm \\
0.08\end{array}$ & $5.01 \times 10^{-3}$ & 0.9408 & $\begin{array}{l}1 \\
2 \\
3 \\
4 \\
5 \\
6\end{array}$ & $\begin{array}{l}-66 \\
-131 \\
-186 \\
-165 \\
-278 \\
-287\end{array}$ & $\begin{array}{l}100.5 \\
106.0 \\
108.1 \\
111.8 \\
108.5 \\
112.1\end{array}$ & $\begin{array}{l}0.227 \\
0.157 \\
0.134 \\
0.134 \\
0.118 \\
0.125\end{array}$ \\
\hline 22 & 0.390 & $100 \pm 0.3$ & $\begin{array}{l}7.60 \pm \\
0.05\end{array}$ & 0.0102 & 0.9895 & $\begin{array}{l}1 \\
2 \\
3 \\
4 \\
5 \\
6\end{array}$ & $\begin{array}{l}-40 \\
-111 \\
-222 \\
-284 \\
-150 \\
-284\end{array}$ & $\begin{array}{l}237.2 \\
258.2 \\
257.0 \\
254.2 \\
258.4 \\
252.9\end{array}$ & $\begin{array}{l}0.0913 \\
0.0101 \\
4.80 \times 10^{-3} \\
2.70 \times 10^{-3} \\
4.50 \times 10^{-3} \\
3.05 \times 10^{-3}\end{array}$ \\
\hline
\end{tabular}




\begin{tabular}{|c|c|c|c|c|c|c|c|c|c|}
\hline Run & $\varepsilon$ & aL & $\frac{\mathrm{q}}{(\mathrm{m} \ell / \min )}$ & $\stackrel{[\mathrm{Cu}}{\left.{ }^{++}\right]_{F}}$ & $\begin{array}{c}{\left[\mathrm{H}_{2} \mathrm{SO}_{4}\right]_{\mathrm{F}}} \\
\underline{\mathrm{M}}\end{array}$ & $\begin{array}{c}\text { Sample } \\
\#\end{array}$ & $\begin{array}{c}\mathrm{V}_{\mathrm{c}}-\phi_{\mathrm{R}} \\
(\mathrm{mV})\end{array}$ & $\begin{array}{c}I_{c} \\
(m A)\end{array}$ & $c_{L} / c_{F}$ \\
\hline 24 & 0.390 & $\begin{array}{c}29.1 \pm \\
0.7\end{array}$ & $\begin{array}{c}10.8 \pm \\
0.0\end{array}$ & 0.0102 & 0.9895 & $\begin{array}{l}1 \\
2 \\
3 \\
4 \\
5 \\
6 \\
7 \\
8\end{array}$ & $\begin{array}{l}-75 \\
-133 \\
-166 \\
-185 \\
-205 \\
-272 \\
-250 \\
-297\end{array}$ & $\begin{array}{l}243.9 \\
264.3 \\
272.4 \\
278.7 \\
283.3 \\
283.9 \\
283.6 \\
288.8\end{array}$ & $\begin{array}{l}0.279 \\
0.231 \\
0.201 \\
0.185 \\
0.169 \\
0.169 \\
0.170 \\
0.171\end{array}$ \\
\hline 25 & 0.393 & $\begin{array}{c}29.7 \pm \\
0.6\end{array}$ & $\begin{array}{l}4.13 \pm \\
0.02\end{array}$ & $9.84 \times 10^{-3}$ & 0.9895 & $\begin{array}{l}1 \\
2 \\
3 \\
4 \\
5 \\
6 \\
7\end{array}$ & $\begin{array}{l}-102 \\
-141 \\
-173 \\
-252 \\
-184 \\
-267 \\
-281\end{array}$ & $\begin{array}{l}111.6 \\
115.2 \\
118.4 \\
116.6 \\
120.7 \\
116.0 \\
119\end{array}$ & $\begin{array}{l}0.207 \\
0.116 \\
0.0922 \\
0.0694 \\
0.141 \\
0.0679 \\
0.0783\end{array}$ \\
\hline 27 & 0.385 & $100 \pm 0.7$ & $\begin{array}{l}3.90 \pm \\
0.02\end{array}$ & 0.0380 & 0.9797 & $\begin{array}{l}1 \\
2 \\
3 \\
4 \\
5 \\
6\end{array}$ & $\begin{array}{l}-51 \\
-100 \\
-140 \\
-176 \\
-210 \\
-246\end{array}$ & $\begin{array}{l}384 \\
453 \\
466 \\
474 \\
477 \\
481\end{array}$ & $\begin{array}{l}0.210 \\
0.0599 \\
0.0298 \\
0.0140 \\
7.49 \times 10^{-3} \\
4.78 \times 10^{-3}\end{array}$ \\
\hline 28 & 0.385 & $100 \pm 0.7$ & $\begin{array}{l}5.73 \pm \\
0.04\end{array}$ & 0.0380 & 0.9797 & $\begin{array}{l}1 \\
2 \\
3 \\
4 \\
5 \\
6 \\
7\end{array}$ & $\begin{array}{l}-49 \\
-100 \\
-145 \\
-175 \\
-205 \\
-245 \\
-275\end{array}$ & $\begin{array}{l}519 \\
650 \\
695 \\
697 \\
701 \\
700 \\
700\end{array}$ & $\begin{array}{l}0.284 \\
0.0850 \\
0.0209 \\
0.0124 \\
8.50 \times 10^{-3} \\
7.45 \times 10^{-3} \\
9.10 \times 10^{-3}\end{array}$ \\
\hline 30 & 0.394 & $\begin{array}{c}29.7 \pm \\
0.7\end{array}$ & $\begin{array}{l}3.78 \pm \\
0.03\end{array}$ & 0.0380 & 0.9797 & $\begin{array}{l}1 \\
2 \\
3 \\
4 \\
5 \\
6\end{array}$ & $\begin{array}{l}-25 \\
-100 \\
-150 \\
-180 \\
-210 \\
-250\end{array}$ & $\begin{array}{l}314 \\
400 \\
418 \\
422 \\
422 \\
423\end{array}$ & $\begin{array}{l}0.316 \\
0.127 \\
0.0916 \\
0.0784 \\
0.0739 \\
0.0655\end{array}$ \\
\hline
\end{tabular}




\begin{tabular}{|c|c|c|c|c|c|c|c|c|c|}
\hline Run & $\varepsilon$ & $\mathrm{aL}$ & $\begin{array}{c}\mathrm{q} \\
(\mathrm{m} \ell / \mathrm{min})\end{array}$ & $\begin{array}{c}{\left[\mathrm{Cu}^{+}\right]_{F}} \\
(\underline{M})\end{array}$ & $\begin{array}{c}{\left[\mathrm{H}_{2} \mathrm{SO}_{4}\right] \mathrm{F}} \\
\underline{\mathrm{M}}\end{array}$ & $\begin{array}{c}\text { Sample } \\
\# \#\end{array}$ & $\begin{array}{c}\mathrm{V}_{c}-\phi_{\mathrm{R}} \\
(\mathrm{mV})\end{array}$ & $\underset{(\mathrm{mA})}{I_{c}}$ & $c_{L} / c_{F}$ \\
\hline 31 & 0.392 & $\begin{array}{c}30.4 \pm \\
0.7\end{array}$ & $\begin{array}{r}14.3 \pm \\
0.05\end{array}$ & 0.0144 & 1.016 & $\begin{array}{l}1 \\
2 \\
3 \\
4 \\
5\end{array}$ & $\begin{array}{l}-100 \\
-116 \\
-145 \\
-176 \\
-196\end{array}$ & $\begin{array}{l}471.3 \\
499.4 \\
519.6 \\
528.8 \\
530.7\end{array}$ & $\begin{array}{l}0.230 \\
0.230 \\
0.213 \\
0.198 \\
0.201\end{array}$ \\
\hline 32 & 0.396 & $\begin{array}{c}30.5 \pm \\
0.9\end{array}$ & $\begin{array}{l}2.15 \pm \\
0.05\end{array}$ & 0.0102 & 0.9796 & $\begin{array}{l}1 \\
2 \\
3 \\
4 \\
5 \\
6\end{array}$ & $\begin{array}{l}-70 \\
-110 \\
-145 \\
-175 \\
-205 \\
-245\end{array}$ & $\begin{array}{l}67.2 \\
68.0 \\
69.4 \\
69.5 \\
70.3 \\
71.5\end{array}$ & $\begin{array}{l}0.0675 \\
0.0494 \\
0.0433 \\
0.0375 \\
0.0308 \\
0.0269\end{array}$ \\
\hline 33 & 0.396 & $\begin{array}{c}30.5 \pm \\
0.9\end{array}$ & $\begin{array}{l}.867 \pm \\
0.018\end{array}$ & 0.0102 & 0.9796 & $\begin{array}{l}1 \\
2 \\
3 \\
4 \\
5 \\
6\end{array}$ & $\begin{array}{l}-30 \\
-75 \\
-115 \\
-155 \\
-190 \\
-235\end{array}$ & $\begin{array}{l}27.2 \\
29.0 \\
28.4 \\
28.9 \\
29.1 \\
26.8\end{array}$ & $\begin{array}{l}7.26 \times 10^{-4} \\
3.17 \times 10^{-4} \\
1.45 \times 10^{-4} \\
1.46 \times 10^{-4} \\
1.04 \times 10^{-4} \\
6.61 \times 10^{-5}\end{array}$ \\
\hline 34 & 0.396 & $\begin{array}{c}30.5 \pm \\
0.9\end{array}$ & $11.8 \pm 0.0$ & 0.0102 & 0.9796 & $\begin{array}{l}1 \\
2 \\
3 \\
4 \\
5 \\
6 \\
7\end{array}$ & $\begin{array}{l}-72 \\
-115 \\
-150 \\
-180 \\
-205 \\
-235 \\
-255\end{array}$ & $\begin{array}{l}328.5 \\
340.0 \\
343.4 \\
345.5 \\
345.8 \\
346.4 \\
347.5\end{array}$ & $\begin{array}{l}0.169 \\
0.138 \\
0.0755 \\
0.118 \\
0.116 \\
0.114 \\
0.112\end{array}$ \\
\hline 35 & 0.392 & $101 \pm 1$ & $\begin{array}{l}2.51 \pm \\
0.02\end{array}$ & 0.0102 & 0.9796 & $\begin{array}{l}1 \\
2 \\
3 \\
4 \\
5 \\
6\end{array}$ & $\begin{array}{l}-75 \\
-120 \\
-155 \\
-180 \\
-205 \\
-235\end{array}$ & $\begin{array}{l}76.5 \\
80.3 \\
82.4 \\
80.5 \\
78.9 \\
75.2\end{array}$ & $\begin{array}{l}0.0928 \\
0.0209 \\
0.0248 \\
1.13 \times 10^{-3} \\
1.82 \times 10^{-3} \\
5.00 \times 10^{-4}\end{array}$ \\
\hline
\end{tabular}




\begin{tabular}{|c|c|c|c|c|c|c|c|c|c|}
\hline Run & $\varepsilon$ & $\mathrm{aL}$ & $\begin{array}{c}\mathrm{q} \\
(\mathrm{m} \ell / \min )\end{array}$ & $\begin{array}{c}{\left[\mathrm{Cu}^{++}\right]_{F}} \\
(\underline{M})\end{array}$ & $\begin{array}{c}{\left[\mathrm{H}_{2} \mathrm{SO}_{4}\right]_{\mathrm{F}}} \\
\underline{\mathrm{M}}\end{array}$ & $\begin{array}{c}\text { Sample } \\
\text { \#⿰ }\end{array}$ & $\begin{array}{c}\mathrm{V}_{\mathrm{c}}-\phi_{\mathrm{R}} \\
(\mathrm{mV})\end{array}$ & $\begin{array}{c}I_{c} \\
(\mathrm{~mA})\end{array}$ & $c_{L} / c_{F}$ \\
\hline 36 & 0.392 & $101 \pm 1$ & $\begin{array}{l}4.50 \pm \\
0.03\end{array}$ & 0.0102 & 0.9796 & $\begin{array}{l}1 \\
2 \\
3 \\
4 \\
5 \\
6\end{array}$ & $\begin{array}{l}-100 \\
-135 \\
-160 \\
-185 \\
-205 \\
-235\end{array}$ & $\begin{array}{l}149.8 \\
149 \\
148.6 \\
146.0 \\
145.6 \\
144\end{array}$ & $\begin{array}{l}4.32 \times 10^{-3} \\
2.71 \times 10^{-3} \\
2.30 \times 10^{-3} \\
1.54 \times 10^{-3} \\
1.82 \times 10^{-3} \\
1.58 \times 10^{-3}\end{array}$ \\
\hline 37 & 0.392 & $101 \pm 1$ & $\begin{array}{c}14.7 \pm \\
0.1\end{array}$ & 0.0102 & 0.9796 & $\begin{array}{l}1 \\
2 \\
3 \\
4 \\
5 \\
6\end{array}$ & $\begin{array}{l}-110 \\
-150 \\
-170 \\
-145 \\
-220 \\
-245\end{array}$ & $\begin{array}{l}469 \\
471 \\
470 \\
470 \\
471 \\
471\end{array}$ & $\begin{array}{l}2.40 \times 10^{-4} \\
1.76 \times 10^{-4} \\
1.59 \times 10^{-4} \\
1.27 \times 10^{-4} \\
1.09 \times 10^{-4} \\
9.86 \times 10^{-5}\end{array}$ \\
\hline 40 & 0.400 & $\begin{array}{c}30.4 \pm \\
0.6\end{array}$ & $\begin{array}{l}6.00 \pm \\
0.02\end{array}$ & $\begin{array}{c}5.14 \pm 0.4 \\
\times 10^{-3}\end{array}$ & 0.9842 & $\begin{array}{l}1 \\
2 \\
3 \\
4 \\
5\end{array}$ & $\begin{array}{l}-160 \\
-185 \\
-210 \\
-235 \\
-255\end{array}$ & $\begin{array}{l}86.8 \\
88.4 \\
88.9 \\
89.4 \\
89.0\end{array}$ & $\begin{array}{l}0.147 \\
0.112 \\
0.115 \\
0.115 \\
0.113\end{array}$ \\
\hline 41 & 0.393 & $\begin{array}{c}99.9 \pm \\
0.5\end{array}$ & $\begin{array}{r}10.7 \pm \\
0.05\end{array}$ & $\begin{array}{l}5.14 \pm .04 \\
\times 10^{-3}\end{array}$ & 0.9842 & $\begin{array}{l}1 \\
2 \\
3 \\
4 \\
5 \\
6\end{array}$ & $\begin{array}{l}-155 \\
-180 \\
-195 \\
-215 \\
-230 \\
-250\end{array}$ & $\begin{array}{l}169.4 \\
173.5 \\
175.6 \\
175.6 \\
175.8 \\
176.5\end{array}$ & $\begin{array}{l}0.0440 \\
0.0344 \\
0.0137 \\
8.77 \times 10^{-3} \\
5.25 \times 10^{-3} \\
3.91 \times 10^{-3}\end{array}$ \\
\hline 42 & 0.393 & $\begin{array}{c}99.9 \pm \\
0.5\end{array}$ & $\begin{array}{l}4.36 \pm \\
0.03\end{array}$ & $\begin{array}{c}5.14 \pm 0.4 \\
\times 10^{-3}\end{array}$ & 0.9842 & $\begin{array}{l}1 \\
2 \\
3 \\
4 \\
5\end{array}$ & $\begin{array}{l}-125 \\
-180 \\
-205 \\
-230 \\
-250\end{array}$ & $\begin{array}{l}72.3 \\
73.3 \\
72.8 \\
72.2 \\
72.3\end{array}$ & $\begin{array}{l}9.72 \times 10^{-4} \\
5.64 \times 10^{-4} \\
2.92 \times 10^{-4} \\
2.33 \times 10^{-4} \\
1.83 \times 10^{-4}\end{array}$ \\
\hline 43 & 0.393 & $\begin{array}{c}99.9 \pm \\
0.5\end{array}$ & $\begin{array}{l}9.00 \pm \\
0.04\end{array}$ & $\begin{array}{c}5.14 \pm 04 \\
\times 10^{-3}\end{array}$ & 0.9842 & $\begin{array}{l}1 \\
2 \\
3 \\
4 \\
5\end{array}$ & $\begin{array}{l}-125 \\
-180 \\
-205 \\
-280 \\
-255\end{array}$ & $\begin{array}{l}148.8 \\
148.8 \\
148.5 \\
148.5 \\
148.4\end{array}$ & $\begin{array}{l}4.05 \times 10^{-3} \\
3.02 \times 10^{-3} \\
2.92 \times 10^{-3} \\
2.51 \times 10^{-3} \\
2.59 \times 10^{-3}\end{array}$ \\
\hline
\end{tabular}




\begin{tabular}{|c|c|c|c|c|c|c|c|c|c|}
\hline Run & $\varepsilon$ & aL & $\begin{array}{c}\mathrm{q} \\
(\mathrm{ml} / \mathrm{min})\end{array}$ & $\begin{array}{c}{\left[\mathrm{Cu}^{++}\right]_{\mathrm{F}}} \\
(\underline{\mathrm{M}})\end{array}$ & $\begin{array}{c}{\left[\mathrm{H}_{2} \mathrm{SO}_{4}\right]_{\mathrm{F}}} \\
\underline{\mathrm{M}}\end{array}$ & $\begin{array}{c}\text { Sample } \\
\#\end{array}$ & $\begin{array}{l}V_{c}-\phi_{R} \\
(m V)\end{array}$ & $\begin{array}{c}I_{C} \\
(m A)\end{array}$ & $c_{L} / c_{F}$ \\
\hline 44 & 0.393 & $\begin{array}{c}99.9 \pm \\
0.5\end{array}$ & $\begin{array}{r}16.0 \pm \\
0.05\end{array}$ & $\begin{array}{l}5.14 \pm .04 \\
\times 10^{-3}\end{array}$ & 0.9842 & $\begin{array}{l}1 \\
2 \\
3 \\
4 \\
5\end{array}$ & $\begin{array}{l}-125 \\
-170 \\
-200 \\
-225 \\
-250\end{array}$ & $\begin{array}{l}260.0 \\
261.6 \\
262.2 \\
262.1 \\
262.3\end{array}$ & $\begin{array}{l}0.0107 \\
0.0125 \\
0.0108 \\
9.59 \times 10^{-3} \\
0.0101\end{array}$ \\
\hline 45 & 0.393 & $\begin{array}{c}99.9 \pm \\
0.5\end{array}$ & $\begin{array}{c}20.0 \pm \\
0.1\end{array}$ & $\begin{array}{l}5.14 \pm .04 \\
\times 10^{-3}\end{array}$ & 0.9842 & $\begin{array}{l}1 \\
2 \\
3 \\
4 \\
5 \\
6\end{array}$ & $\begin{array}{l}-100 \\
-165 \\
-190 \\
-210 \\
-230 \\
-250\end{array}$ & $\begin{array}{l}324.3 \\
326.4 \\
327.0 \\
327.7 \\
327.0 \\
327.1\end{array}$ & $\begin{array}{l}0.0302 \\
0.0204 \\
0.0162 \\
0.0150 \\
0.0161 \\
0.0134\end{array}$ \\
\hline 47 & 0.393 & $\begin{array}{c}99.9 \pm \\
0.5\end{array}$ & $\begin{array}{c}29.7 \pm \\
0.2\end{array}$ & $\begin{array}{c}5.14 \pm .04 \\
\times 10^{-3}\end{array}$ & 0.9842 & $\begin{array}{l}1 \\
2 \\
3 \\
4 \\
5 \\
6\end{array}$ & $\begin{array}{l}-75 \\
-150 \\
-185 \\
-205 \\
-225 \\
-250\end{array}$ & $\begin{array}{l}445.0 \\
447.2 \\
476.3 \\
479.5 \\
480.7 \\
480.9\end{array}$ & $\begin{array}{l}0.102 \\
0.0492 \\
0.0387 \\
0.0368 \\
0.0323 \\
0.0309\end{array}$ \\
\hline 48 & 0.393 & $\begin{array}{c}99.9 \pm \\
0.5\end{array}$ & $\begin{array}{c}33.9 \pm \\
0.4\end{array}$ & $\begin{array}{c}5.14 \pm .04 \\
\times 10^{-3}\end{array}$ & 0.9842 & $\begin{array}{l}1 \\
2 \\
3 \\
4 \\
5 \\
6 \\
7\end{array}$ & $\begin{array}{l}-100 \\
-165 \\
-190 \\
-210 \\
-230 \\
-250 \\
-270\end{array}$ & $\begin{array}{l}511.5 \\
531.1 \\
535.5 \\
538.7 \\
541.0 \\
543.3 \\
546.0\end{array}$ & $\begin{array}{l}0.0856 \\
0.0539 \\
0.0463 \\
0.0424 \\
0.0411 \\
0.0379 \\
0.0366\end{array}$ \\
\hline 51 & 0.373 & $\begin{array}{c}30.3 \pm \\
0.8\end{array}$ & $\begin{array}{l}42.5 \pm \\
0.4\end{array}$ & $\begin{array}{l}1.02 \pm .005 \\
\times 10^{-3}\end{array}$ & 0.9916 & $\begin{array}{l}1 \\
2 \\
3 \\
4 \\
5 \\
6\end{array}$ & $\begin{array}{l}-90 \\
-180 \\
-200 \\
-220 \\
-240 \\
-260\end{array}$ & $\begin{array}{l}77.6 \\
84.3 \\
86.1 \\
87.0 \\
87.5 \\
88.1\end{array}$ & $\begin{array}{l}0.464 \\
0.429 \\
0.443 \\
0.432 \\
0.415 \\
0.414\end{array}$ \\
\hline
\end{tabular}




\begin{tabular}{|c|c|c|c|c|c|c|c|c|c|}
\hline Run & $\varepsilon$ & aL & $\begin{array}{c}\mathrm{q} \\
(\mathrm{m} \ell / \mathrm{min})\end{array}$ & $\begin{array}{c}{\left[\mathrm{Cu}^{++}\right]_{F}} \\
(\underline{M})\end{array}$ & $\begin{array}{c}{\left[\mathrm{H}_{2} \mathrm{SO}_{4}\right]_{\mathrm{F}}} \\
\underline{\mathrm{M}}\end{array}$ & $\begin{array}{c}\text { Sample } \\
\#\end{array}$ & $\begin{array}{c}\mathrm{v}_{\mathrm{c}}-\phi_{\mathrm{R}} \\
(\mathrm{mV})\end{array}$ & $\begin{array}{c}I_{c} \\
(m A)\end{array}$ & $c_{L} / c_{F}$ \\
\hline 52 & 0.373 & $\begin{array}{c}30.3 \pm \\
0.8\end{array}$ & $\begin{array}{l}49.2 \pm \\
0.3\end{array}$ & $\begin{array}{l}1.02 \pm 005 \\
\times 10^{-3}\end{array}$ & 0.9916 & $\begin{array}{l}1 \\
2 \\
3 \\
4 \\
5 \\
6\end{array}$ & $\begin{array}{l}-95 \\
-170 \\
-200 \\
-220 \\
-240 \\
-260\end{array}$ & $\begin{array}{l}86.0 \\
92.0 \\
93.6 \\
94.7 \\
95.4 \\
96.0\end{array}$ & $\begin{array}{l}0.513 \\
0.468 \\
0.454 \\
0.455 \\
0.447 \\
0.436\end{array}$ \\
\hline 53 & 0.373 & $\begin{array}{c}30.3 \pm \\
0.8\end{array}$ & $\begin{array}{c}58.6 \pm \\
0.5\end{array}$ & $\begin{array}{l}1.02 \pm .005 \\
\times 10^{-3}\end{array}$ & 0.9916 & $\begin{array}{l}1 \\
2 \\
3 \\
4 \\
5 \\
6 \\
7\end{array}$ & $\begin{array}{l}-100 \\
-185 \\
-205 \\
-225 \\
-250 \\
-275 \\
-300\end{array}$ & $\begin{array}{r}97.0 \\
104.2 \\
105.2 \\
106.2 \\
107.2 \\
108.3 \\
109.4\end{array}$ & $\begin{array}{l}0.552 \\
0.481 \\
0.477 \\
0.473 \\
0.466 \\
0.466 \\
0.467\end{array}$ \\
\hline 55 & 0.383 & $100 \pm 1$ & $\begin{array}{c}25.1 \pm \\
0.2\end{array}$ & $\begin{array}{l}1.83 \pm .02 \\
\times 10^{-3}\end{array}$ & 1.019 & $\begin{array}{l}1 \\
2 \\
3 \\
4 \\
5 \\
6\end{array}$ & $\begin{array}{l}-80 \\
-190 \\
-220 \\
-250 \\
-275 \\
-300\end{array}$ & $\begin{array}{l}157.4 \\
163.4 \\
162.6 \\
161.7 \\
160.5 \\
164.4\end{array}$ & $\begin{array}{l}0.0913 \\
0.0362 \\
0.0334 \\
0.0320 \\
0.0328 \\
0.0327\end{array}$ \\
\hline 56 & 0.383 & $100 \pm 1$ & $\begin{array}{c}44.7 \pm \\
0.3\end{array}$ & $\begin{array}{c}1.83 \pm .02 \\
\times 10^{-3}\end{array}$ & 1.019 & $\begin{array}{l}1 \\
2 \\
3 \\
4 \\
5 \\
6\end{array}$ & $\begin{array}{l}-80 \\
-190 \\
-220 \\
-250 \\
-275 \\
-295\end{array}$ & $\begin{array}{l}247.5 \\
271.3 \\
273.2 \\
274.6 \\
276.7 \\
279.3\end{array}$ & $\begin{array}{l}0.172 \\
0.0934 \\
0.0880 \\
0.0863 \\
0.0825 \\
0.0820\end{array}$ \\
\hline 57 & 0.383 & $100 \pm 1$ & $\begin{array}{l}36.5 \pm \\
0.2\end{array}$ & $\begin{array}{c}1.83 \pm .02 \\
\times 10^{-3}\end{array}$ & 1.019 & $\begin{array}{l}1 \\
2 \\
3 \\
4 \\
5 \\
6\end{array}$ & $\begin{array}{l}-80 \\
-195 \\
-225 \\
-250 \\
-275 \\
-300\end{array}$ & $\begin{array}{l}220.5 \\
231.7 \\
231.4 \\
231.4 \\
232.0 \\
235.1\end{array}$ & $\begin{array}{l}0.124 \\
0.0659 \\
0.0634 \\
0.0612 \\
0.0596 \\
0.0590\end{array}$ \\
\hline
\end{tabular}




\begin{tabular}{|c|c|c|c|c|c|c|c|c|c|}
\hline Run & $\varepsilon$ & $a L$ & $\begin{array}{c}q \\
(m \ell / \min )\end{array}$ & $\begin{array}{c}{\left[\mathrm{Cu}^{++}\right]_{\mathrm{F}}} \\
(\underline{\mathrm{M}})\end{array}$ & $\begin{array}{c}{\left[\mathrm{H}_{2} \mathrm{SO}_{4}\right] \mathrm{F}} \\
\underline{\mathrm{M}}\end{array}$ & $\begin{array}{c}\text { Sample } \\
\quad \#\end{array}$ & $\begin{array}{c}\mathrm{V}_{\mathrm{c}}-\phi_{\mathrm{R}} \\
(\mathrm{mV})\end{array}$ & $\begin{array}{c}I_{c} \\
(m A)\end{array}$ & $c_{L} / c_{F}$ \\
\hline 58 & 0.383 & $100 \pm 1$ & $\begin{array}{c}50.6 \pm \\
0.3\end{array}$ & $\begin{array}{c}1.83 \pm .02 \\
\times 10^{-3}\end{array}$ & 1.019 & $\begin{array}{l}1 \\
2 \\
3 \\
4 \\
5 \\
6\end{array}$ & $\begin{array}{l}-80 \\
-190 \\
-220 \\
-245 \\
-270 \\
-290\end{array}$ & $\begin{array}{l}284.8 \\
305.5 \\
307.8 \\
309.8 \\
312.5 \\
315.6\end{array}$ & $\begin{array}{l}0.161 \\
0.0967 \\
0.0913 \\
0.0863 \\
0.0874 \\
0.0847\end{array}$ \\
\hline 59 & 0.383 & $100 \pm 1$ & $\begin{array}{c}63.9 \pm \\
0.4\end{array}$ & $\begin{array}{c}1.83 \pm .02 \\
\times 10^{-3}\end{array}$ & 1.019 & $\begin{array}{l}1 \\
2 \\
3 \\
4 \\
5 \\
6\end{array}$ & $\begin{array}{l}-90 \\
-190 \\
-220 \\
-245 \\
-270 \\
-300\end{array}$ & $\begin{array}{l}340.9 \\
372.7 \\
377.4 \\
381.6 \\
386.0 \\
393.0\end{array}$ & $\begin{array}{l}0.192 \\
0.127 \\
0.120 \\
0.115 \\
0.110 \\
0.108\end{array}$ \\
\hline 61 & 0.400 & $\begin{array}{c}29.9 \pm \\
0.9\end{array}$ & $\begin{array}{l}5.04 \pm \\
0.02\end{array}$ & $\begin{array}{l}7.91 \pm .05 \\
\times 10^{-3}\end{array}$ & $\begin{array}{c}1.000 \\
.\end{array}$ & $\begin{array}{l}1 \\
2 \\
3 \\
4 \\
5 \\
6\end{array}$ & $\begin{array}{l}-90 \\
-180 \\
-215 \\
-245 \\
-270 \\
-230\end{array}$ & $\begin{array}{l}110.2 \\
117.4 \\
118.7 \\
118.6 \\
120.2 \\
119.2\end{array}$ & $\begin{array}{l}0.185 \\
0.117 \\
0.0805 \\
0.0697 \\
0.0576 \\
0.0598\end{array}$ \\
\hline 62 & 0.400 & $\begin{array}{c}29.9 \pm \\
0.9\end{array}$ & $\begin{array}{l}1.41 \pm \\
0.02\end{array}$ & $\begin{array}{l}7.91 \pm .05 \\
\times 10^{-3}\end{array}$ & 1.000 & $\begin{array}{l}1 \\
2 \\
3 \\
4 \\
5 \\
6\end{array}$ & $\begin{array}{l}-90 \\
-195 \\
-225 \\
-245 \\
-265 \\
-290\end{array}$ & $\begin{array}{l}35.5 \\
36.0 \\
35.4 \\
34.8 \\
35.0 \\
36.6\end{array}$ & $\begin{array}{l}0.0253 \\
0.0140 \\
0.0134 \\
0.00813 \\
0.00786 \\
0.0108\end{array}$ \\
\hline 63 & 0.400 & $\begin{array}{c}29.9 \pm \\
0.9\end{array}$ & $\begin{array}{l}6.85 \pm \\
0.04\end{array}$ & $\begin{array}{l}7.91 \pm .05 \\
\times 10^{-3}\end{array}$ & 1.000 & $\begin{array}{l}1 \\
2 \\
3 \\
4 \\
5 \\
6\end{array}$ & $\begin{array}{l}-90 \\
-190 \\
-215 \\
-245 \\
-265 \\
-295\end{array}$ & $\begin{array}{l}154.1 \\
160.7 \\
162.5 \\
163.0 \\
162.5 \\
163.1\end{array}$ & $\begin{array}{l}0.123 \\
0.0845 \\
0.0638 \\
0.0625 \\
0.0589 \\
0.0551\end{array}$ \\
\hline
\end{tabular}




\begin{tabular}{|c|c|c|c|c|c|c|c|c|c|}
\hline Run & $\varepsilon$ & aL & $\begin{array}{c}\mathrm{q} \\
(\mathrm{ml} / \mathrm{min})\end{array}$ & $\begin{array}{c}{\left[\mathrm{Cu}^{++}\right]_{F}} \\
(\underline{M})\end{array}$ & $\begin{array}{c}{\left[\mathrm{H}_{2} \mathrm{SO}_{4}\right]_{\mathrm{F}}} \\
\underline{\mathrm{M}}\end{array}$ & $\begin{array}{c}\text { Sample } \\
\# \equiv\end{array}$ & $\begin{array}{c}\mathrm{V}_{\mathrm{c}}{ }^{-\phi} \mathrm{R} \\
(\mathrm{mV})\end{array}$ & $\underset{(\mathrm{mA})}{\mathrm{I}_{c}}$ & $c_{L} / c_{F}$ \\
\hline 64 & 0.400 & $\begin{array}{c}29.9 \pm \\
0.9\end{array}$ & $\begin{array}{l}2.99 \pm \\
0.03\end{array}$ & $\begin{array}{l}7.91 \pm .05 \\
\times 10^{-3}\end{array}$ & 1.000 & $\begin{array}{l}1 \\
2 \\
3 \\
4 \\
5 \\
6\end{array}$ & $\begin{array}{l}-90 \\
-200 \\
-225 \\
-245 \\
-270 \\
-300\end{array}$ & $\begin{array}{l}73.8 \\
74.5 \\
74.7 \\
74.2 \\
74.0 \\
76.2\end{array}$ & $\begin{array}{l}0.0359 \\
0.0193 \\
0.0192 \\
0.0214 \\
0.0181 \\
0.0158\end{array}$ \\
\hline 65 & 0.400 & $\begin{array}{c}29.9 \pm \\
0.9\end{array}$ & $\begin{array}{c}12.3 \pm \\
0.1\end{array}$ & $\begin{array}{c}7.91 \pm .05 \\
\times 10^{-3}\end{array}$ & 1.000 & $\begin{array}{l}1 \\
2 \\
3 \\
4 \\
5 \\
6\end{array}$ & $\begin{array}{l}-90 \\
-190 \\
-220 \\
-245 \\
-270 \\
-295\end{array}$ & $\begin{array}{l}249.6 \\
268.5 \\
269.9 \\
270.8 \\
271.5 \\
272.8\end{array}$ & $\begin{array}{l}0.159 \\
0.134 \\
0.126 \\
0.120 \\
0.118 \\
0.114\end{array}$ \\
\hline 66 & 0.400 & $\begin{array}{c}29.9 \pm \\
0.9\end{array}$ & $\begin{array}{c}18.2 \pm \\
0.1\end{array}$ & $\begin{array}{l}7.91 \pm .05 \\
\times 10^{-3}\end{array}$ & 1.000 & $\begin{array}{l}1 \\
2 \\
3 \\
4 \\
5 \\
6\end{array}$ & $\begin{array}{l}-90 \\
-190 \\
-220 \\
-245 \\
-275 \\
-310\end{array}$ & $\begin{array}{l}347.0 \\
372.3 \\
375.5 \\
376.6 \\
378.3 \\
381.8\end{array}$ & $\begin{array}{l}0.230 \\
0.212 \\
0.188 \\
0.186 \\
0.183 \\
0.178\end{array}$ \\
\hline 67 & 0.387 & $100 \pm 1$ & $\begin{array}{l}3.00 \pm \\
0.02\end{array}$ & $\begin{array}{l}7.91 \pm .05 \\
\times 10^{-3}\end{array}$ & 1.000 & $\begin{array}{l}1 \\
2 \\
3 \\
4 \\
5 \\
6\end{array}$ & $\begin{array}{l}-90 \\
-190 \\
-225 \\
-250 \\
-275 \\
-305\end{array}$ & $\begin{array}{l}75.0 \\
76.3 \\
76.4 \\
77.4 \\
76.7 \\
76.6\end{array}$ & $\begin{array}{l}0.0244 \\
2.64 \times 10^{-3} \\
1.83 \times 10^{-3} \\
1.02 \times 10^{-3} \\
1.13 \times 10^{-3} \\
7.59 \times 10^{-4}\end{array}$ \\
\hline 68 & 0.387 & $100 \pm 1$ & $\begin{array}{l}1.93 \pm \\
0.06\end{array}$ & $\begin{array}{l}7.91 \pm .05 \\
\times 10^{-3}\end{array}$ & 1.000 & $\begin{array}{l}1 \\
2 \\
3 \\
4 \\
5\end{array}$ & $\begin{array}{l}-90 \\
-210 \\
-240 \\
-270 \\
-300\end{array}$ & $\begin{array}{l}47.8 \\
48.4 \\
52.8 \\
49.5 \\
51.3\end{array}$ & $\begin{array}{l}1.99 \times 10^{-3} \\
1.90 \times 10^{-4} \\
3.67 \times 10^{-4} \\
6.19 \times 10^{-4} \\
2.40 \times 10^{-4}\end{array}$ \\
\hline
\end{tabular}




\begin{tabular}{|c|c|c|c|c|c|c|c|c|c|}
\hline Run & $\varepsilon$ & aL & $\begin{array}{c}q \\
(\mathrm{~m} \ell / \mathrm{min})\end{array}$ & $\begin{array}{c}{\left[\mathrm{Cu}^{++}\right]_{F}} \\
\stackrel{(M)}{ }\end{array}$ & $\begin{array}{c}{\left[\mathrm{H}_{2} \mathrm{SO}_{4}\right] \mathrm{F}} \\
\underline{\mathrm{M}}\end{array}$ & $\begin{array}{c}\text { Sample } \\
\# \equiv\end{array}$ & $\begin{array}{c}\mathrm{V}_{\mathrm{c}}{ }^{-\phi_{\mathrm{R}}} \\
(\mathrm{mV})\end{array}$ & $\underset{(\mathrm{mA})}{I_{c}}$ & $c_{L} / c_{F}$ \\
\hline 69 & 0.387 & $100 \pm 1$ & $\begin{array}{l}5.97 \pm \\
0.07\end{array}$ & $\begin{array}{l}7.91 \pm .05 \\
\times 10^{-3}\end{array}$ & 1.000 & $\begin{array}{l}1 \\
2 \\
3 \\
4 \\
5 \\
6\end{array}$ & $\begin{array}{l}-90 \\
-190 \\
-225 \\
-250 \\
-275 \\
-300\end{array}$ & $\begin{array}{l}150.8 \\
151.8 \\
151.3 \\
149.1 \\
146.3 \\
151.2\end{array}$ & $\begin{array}{l}9.72 \times 10^{-3} \\
4.86 \times 10^{-3} \\
3.15 \times 10^{-3} \\
3.22 \times 10^{-3} \\
3.19 \times 10^{-3} \\
3.86 \times 10^{-3}\end{array}$ \\
\hline 70 & 0.387 & $100 \pm 1$ & $\begin{array}{l}8.29 \pm \\
0.04\end{array}$ & $\begin{array}{l}7.91 \pm .05 \\
\times 10^{-3}\end{array}$ & 1.000 & $\begin{array}{l}1 \\
2 \\
3 \\
4 \\
5 \\
6\end{array}$ & $\begin{array}{l}-90 \\
-190 \\
-225 \\
-250 \\
-275 \\
-310\end{array}$ & $\begin{array}{l}207.8 \\
209.1 \\
208.2 \\
208.8 \\
208.6 \\
210.2\end{array}$ & $\begin{array}{l}0.0144 \\
6.14 \times 10^{-3} \\
2.57 \times 10^{-3} \\
4.97 \times 10^{-3} \\
5.70 \times 10^{-3} \\
4.51 \times 10^{-3}\end{array}$ \\
\hline 71 & 0.387 & $100 \pm 1$ & $\begin{array}{c}12.3 \pm \\
0.1\end{array}$ & $\begin{array}{l}7.91 \pm .05 \\
\times 10^{-3}\end{array}$ & 1.000 & $\begin{array}{l}1 \\
2 \\
3 \\
4 \\
5 \\
6\end{array}$ & $\begin{array}{l}-90 \\
-190 \\
-225 \\
-250 \\
-275 \\
-305\end{array}$ & $\begin{array}{l}297.6 \\
303.9 \\
305.5 \\
305.8 \\
305.4 \\
305.2\end{array}$ & $\begin{array}{l}0.0224 \\
7.96 \times 10^{-3} \\
9.63 \times 10^{-3} \\
9.27 \times 10^{-3} \\
9.09 \times 10^{-3} \\
8.93 \times 10^{-3}\end{array}$ \\
\hline 72 & 0.387 & $100 \pm 1$ & $\begin{array}{l}7.09 \pm \\
0.05\end{array}$ & $\begin{array}{l}7.88 \pm .06 \\
\times 10^{-3}\end{array}$ & 1.000 & $\begin{array}{l}1 \\
2 \\
3 \\
4 \\
5 \\
6\end{array}$ & $\begin{array}{l}-90 \\
-190 \\
-225 \\
-250 \\
-275 \\
-310\end{array}$ & $\begin{array}{l}179.0 \\
181.0 \\
181.5 \\
182.4 \\
183.5 \\
182.0\end{array}$ & $\begin{array}{l}9.59 \times 10^{-3} \\
3.41 \times 10^{-3} \\
2.63 \times 10^{-3} \\
2.04 \times 10^{-3} \\
2.79 \times 10^{-3} \\
2.82 \times 10^{-3}\end{array}$ \\
\hline
\end{tabular}

Runs 74 Through 83 Contain 2.99 M Glycerol

\begin{tabular}{|c|c|c|c|c|c|c|c|c|}
\hline 0.388 & $\begin{array}{c}30.0 \pm \\
0.7\end{array}$ & $\begin{array}{l}4.06 \pm \\
0.04\end{array}$ & $\begin{array}{l}4.99 \pm .01 \\
\times 10^{-3}\end{array}$ & 1.434 & $\begin{array}{l}1 \\
2 \\
3 \\
4 \\
5 \\
6\end{array}$ & $\begin{array}{l}-90 \\
-190 \\
-225 \\
-250 \\
-275 \\
-300\end{array}$ & $\begin{array}{l}57.3 \\
58.0 \\
58.4 \\
59.0 \\
59.4 \\
59.8\end{array}$ & $\begin{array}{l}0.148 \\
0.126 \\
0.112 \\
0.105 \\
0.101 \\
0.0978\end{array}$ \\
\hline
\end{tabular}




\begin{tabular}{|c|c|c|c|c|c|c|c|c|c|}
\hline Run & $\varepsilon$ & aL & $\begin{array}{c}\mathrm{q} \\
(\mathrm{m} \ell / \mathrm{min})\end{array}$ & $\begin{array}{c}{\left[\mathrm{Cu}^{++}\right]_{F}} \\
(\underline{M})\end{array}$ & $\begin{array}{c}{\left[\mathrm{H}_{2} \mathrm{SO}_{4}\right]_{\mathrm{F}}} \\
\underline{\mathrm{M}}\end{array}$ & 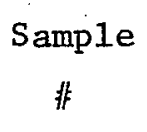 & $\begin{array}{c}\mathrm{V}_{\mathrm{c}}-\phi_{\mathrm{R}} \\
(\mathrm{mV})\end{array}$ & $\underset{(\mathrm{mA})}{\mathrm{I}_{\mathrm{c}}}$ & $c_{L} / c_{F}$ \\
\hline 75 & 0.388 & $\begin{array}{c}30.0 \pm \\
0.7\end{array}$ & $\begin{array}{l}1.72 \pm \\
0.03\end{array}$ & $\begin{array}{l}4.99 \pm .01 \\
\times 10^{-3}\end{array}$ & 1.434 & $\begin{array}{l}1 \\
2 \\
3 \\
4 \\
5 \\
6\end{array}$ & $\begin{array}{l}-90 \\
-190 \\
-225 \\
-250 \\
-275 \\
-300\end{array}$ & $\begin{array}{l}26.7 \\
27.3 \\
27.7 \\
28.0 \\
27.8 \\
28.6\end{array}$ & $\begin{array}{l}0.0537 \\
0.0387 \\
0.0337 \\
0.0335 \\
0.0327 \\
0.0327\end{array}$ \\
\hline 76 & 0.388 & $\begin{array}{c}30.0 \pm \\
0.7\end{array}$ & $\begin{array}{l}5.76 \pm \\
0.03\end{array}$ & $\begin{array}{l}4.99 \pm .01 \\
\times 10^{-3}\end{array}$ & 1.434 & $\begin{array}{l}1 \\
2 \\
3 \\
4 \\
5 \\
6\end{array}$ & $\begin{array}{l}-90 \\
-190 \\
-225 \\
-250 \\
-275 \\
-300\end{array}$ & $\begin{array}{l}76.6 \\
80.0 \\
79.5 \\
80.2 \\
80.4 \\
80.6\end{array}$ & $\begin{array}{l}0.175 \\
0.151 \\
0.155 \\
0.146 \\
0.147 \\
0.144\end{array}$ \\
\hline 77 & 0.388 & $\begin{array}{c}30.0 \pm \\
0.7\end{array}$ & $\begin{array}{l}7.62 \pm \\
0.03\end{array}$ & $\begin{array}{l}4.99 \pm .01 \\
\times 10^{-3}\end{array}$ & 1.434 & $\begin{array}{l}1 \\
2 \\
3 \\
4 \\
5 \\
6\end{array}$ & $\begin{array}{l}-90 \\
-190 \\
-225 \\
-250 \\
-275 \\
-300\end{array}$ & $\begin{array}{r}90.9 \\
97.9 \\
98.2 \\
99.0 \\
99.5 \\
100.0\end{array}$ & $\begin{array}{l}0.240 \\
0.204 \\
0.204 \\
0.191 \\
0.190 \\
0.196\end{array}$ \\
\hline 78 & 0.388 & $\begin{array}{c}30.0 \pm \\
0.7\end{array}$ & $\begin{array}{l}9.43 \pm \\
0.03\end{array}$ & $\begin{array}{l}4.99 \pm .01 \\
\times 10^{-3}\end{array}$ & 1.434 & $\begin{array}{l}1 \\
2 \\
3 \\
4 \\
5 \\
6\end{array}$ & $\begin{array}{l}-90 \\
-190 \\
-225 \\
-255 \\
-285 \\
-310\end{array}$ & $\begin{array}{l}107.0 \\
114.5 \\
115.5 \\
116.1 \\
116.6 \\
117.6\end{array}$ & $\begin{array}{l}0.269 \\
0.240 \\
0.240 \\
0.226 \\
0.226 \\
0.222\end{array}$ \\
\hline 79 & 0.388 & $\begin{array}{c}30.0 \pm \\
0.7\end{array}$ & $\begin{array}{r}11.8 \pm \\
0.04\end{array}$ & $\begin{array}{l}4.99 \pm .01 \\
\times 10^{-3}\end{array}$ & 1.434 & $\begin{array}{l}1 \\
2 \\
3 \\
4 \\
5 \\
6\end{array}$ & $\begin{array}{l}-90 \\
-190 \\
-225 \\
-250 \\
-275 \\
-305\end{array}$ & $\begin{array}{l}125.1 \\
133.7 \\
135.0 \\
135.6 \\
136.1 \\
137.2\end{array}$ & $\begin{array}{l}0.301 \\
0.291 \\
0.277 \\
0.275 \\
0.269 \\
0.267\end{array}$ \\
\hline
\end{tabular}




\begin{tabular}{|c|c|c|c|c|c|c|c|c|c|}
\hline Run & $\varepsilon$ & $\mathrm{aL}$ & $\frac{\mathrm{q}}{(\mathrm{ml} / \mathrm{min})}$ & $\underset{(\underline{M})}{\left[\mathrm{Cu}^{++}\right]_{\mathrm{F}}}$ & $\begin{array}{c}{\left[\mathrm{H}_{2} \mathrm{SO}_{4}\right]_{\mathrm{F}}} \\
\underline{\mathrm{M}}\end{array}$ & $\begin{array}{c}\text { Sample } \\
\text { \# }\end{array}$ & $\begin{array}{c}V_{c}-\phi_{R} \\
(\mathrm{mV})\end{array}$ & $\begin{array}{c}I_{c} \\
(m A)\end{array}$ & $c_{L} / c_{F}$ \\
\hline 80 & 0.388 & $\begin{array}{c}30.0 \pm \\
0.7\end{array}$ & $\begin{array}{c}15.1 \pm \\
0.1\end{array}$ & $\begin{array}{l}4.99 \pm .01 \\
\times 10^{-3}\end{array}$ & 1.434 & $\begin{array}{l}1 \\
2 \\
3 \\
4 \\
5 \\
6\end{array}$ & $\begin{array}{l}-90 \\
-190 \\
-225 \\
-250 \\
-275 \\
-305\end{array}$ & $\begin{array}{l}152.5 \\
164.3 \\
165.5 \\
166.1 \\
166.6 \\
167.6\end{array}$ & $\begin{array}{l}0.369 \\
0.321 \\
0.309 \\
0.307 \\
0.301 \\
0.299\end{array}$ \\
\hline 81 & 0.388 & $\begin{array}{c}30.0 \pm \\
0.7\end{array}$ & $\begin{array}{c}19.8 \pm \\
0.1\end{array}$ & $\begin{array}{c}4.99 \pm .01 \\
\times 10^{-3}\end{array}$ & 1.434 & $\begin{array}{l}1 \\
2 \\
3 \\
4 \\
5 \\
6\end{array}$ & $\begin{array}{l}-90 \\
-190 \\
-225 \\
-250 \\
-275 \\
-300\end{array}$ & $\begin{array}{l}173.7 \\
195.2 \\
197.0 \\
198.7 \\
200.5 \\
201.8\end{array}$ & $\begin{array}{l}0.433 \\
0.391 \\
0.383 \\
0.369 \\
0.365 \\
0.363\end{array}$ \\
\hline 82 & 0.388 & $\begin{array}{c}30.0 \pm \\
0.7\end{array}$ & $\begin{array}{c}25.7 \pm \\
0.2\end{array}$ & $\begin{array}{l}4.99 \pm .01 \\
\times 10^{-3}\end{array}$ & 1.434 & $\begin{array}{l}1 \\
2 \\
3 \\
4 \\
5 \\
6\end{array}$ & $\begin{array}{l}-90 \\
-190 \\
-225 \\
-250 \\
-275 \\
-305\end{array}$ & $\begin{array}{l}202.2 \\
228.9 \\
232.6 \\
234.2 \\
236.0 \\
238.2\end{array}$ & $\begin{array}{l}0.479 \\
0.441 \\
0.425 \\
0.419 \\
0.419 \\
0.423\end{array}$ \\
\hline 83 & 0.388 & $\begin{array}{c}30.0 \pm \\
0.7\end{array}$ & $\begin{array}{c}33.6 \pm \\
0.1\end{array}$ & $\begin{array}{l}4.99 \pm .01 \\
\times 10^{-3}\end{array}$ & 1.434 & $\begin{array}{l}1 \\
2 \\
3 \\
4 \\
5 \\
6 \\
7\end{array}$ & $\begin{array}{l}-100 \\
-200 \\
-240 \\
-275 \\
-300 \\
-335 \\
-365\end{array}$ & $\begin{array}{l}237.5 \\
269.3 \\
274.1 \\
276.6 \\
278.1 \\
281.3 \\
286.3\end{array}$ & $\begin{array}{l}0.551 \\
0.503 \\
0.485 \\
0.473 \\
0.473 \\
0.461 \\
0.471\end{array}$ \\
\hline
\end{tabular}




\section{REFERENCES}

1. Brenner, H., "Rheology of Two-Phase Systems," Advances in F1uid Mechanics, 2, 137 (1970).

2. Nayak, A. L., and C. L. Tien, "A Statistical Thermodynamic Theory for Coordination-Number Distribution and Effective Therma1 Conductivity of Random Packed Beds," Int. J. Heat Mass Transfer, 21, 669 (1978).

3. Haughey, D. P., and G. S. Beveridge, "Structural Properties of Packed Beds - A Review," Can. J. Chem. Eng., 47, 130 (1969).

4. Haughey, D. P., and G. S. Beveridge, "Local Voidage Variation In a Randomly Packed Bed of Equal-Sized Spheres," Chem. Eng. Sci., 21, 905 (1966).

5. Dunning, J. S., Analysis of Porous Electrodes with Sparingly Soluble Reactants, dissertation, University of California, Los Angeles (1971).

6. Slattery, J. C., "Flow of Viscoelastic Flufds Through Porous Media," AIChE J., 13, 1066 (1967).

7. Slattery, J. C., "Single-Phase Flow Through Porous Media," AIChE J., 15, 866 (1969).

8. Whitaker, S., "Advances in Theory of Fluid Motion in Porous Media," Ind. Eng. Chem., 61, no. 12, 14 (1969).

9. Gray, W. G., "A Derivation of the Equations for Multi-Phase Transport," Chem. Eng. Sci., 30, 229 (1975). 
10. Sørensen, J. P., and W. E. Stewart, "Computation of Forced Convection in Slow Flow Through Ducts and Packed Beds - I Extensions of the Graetz Problem," Chem. Eng. Sci., 29, 811 (1974).

11. Sфrensen, J. P., and W. E. Stewart, "Computation of Forced Convection in Slow Flow through Ducts and Packed Beds - II Velocity Profile in a Simple Cubic Array of Spheres," Chem. Eng. Sci., 29, 819 (1974).

12. S $\phi$ rensen, J. P.; and W. E. Stewart, "Computation of Slow Flow through Ducts and Packed Beds - III Heat and Mass Transfer in a Simple Cubic Array of Spheres," Chem. Eng. Sci., 29, 827 (1974).

13. Neale, G. H., and W. K. Nader, "Prediction of Transport Processes within Porous Media: Diffusive Flow Processes within a Homogeneous Swarm of Spherical Particles," AIChE J., 19, 112 (1973).

14. Dullien, F. A. L., "Single Phase Flow Through Porous Media and Pore Structure," Chem. Eng. J., 10, 1 (1975).

15. Pfeffer, R., and $J_{\circ}$ Happe1, "An Analytic Study of Heat and Mass Transfer in Multiparticle Systems at Low Reynolds Numbers," AIChE J., 10, 605 (1964).

16. Appe1, P. W., Electrochemica1 Systems: Impedance of a Rotating

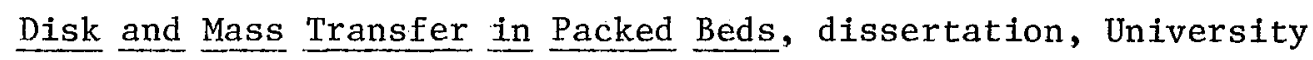
of California, Berkeley (1976).

17. Nelson, P. A., and T. R. Galloway, "Particle-to-Fluid Heat and Mass Transfer in Dense Systems of Fine Particles," Chem. Eng. Sci., 30, 1 (1975). 
18. Pfeffer, R., "Heat and Mass Transport in Multi-Particle Systems," Ind. Eng. Chem. Fund., 3, 383 (1964).

19. E1-Kaissy, M. M., and G. M. Homsy, "A Theoretica1 Study of Pressure Drop and Transportation in Packed Beds at Intermediate Reyno1ds Numbers," Ind. Eng. Chem. Fund., 12, 82 (1973).

20. Brinkman, H. C., "A Calculation of the Viscous Force Exerted by a Flowing Fluid in a Dense Swarm of Particles," Appl. Sci. Res., A1, 27 (1947).

21. Tardos, G. I., Gutfinger, C., and N. Abuaf, "High Péclet Number Mass Transfer to a Sphere in a Fixed and Fluidized Bed," AIChE J., 22, 1147 (1976).

22. Neale, G. H., and W. K. Nader, "Prediction of Transport Processes within Porous Media: Creeping Flow Relative to a Fixed Swarm of Spherical Particles," AIChE J., 20, 530 (1974).

23. Sheidegger, A. E., The Physics of Flow Through Porous Media, Macmillan Co., N.Y., 1957.

24. Bear, J., Dynamics of Fluids in Porous Media, E1sevier, New York, 1972.

25. Kataoka, T., Yoshida, H., and K. Veyama, "Mass Transfer in Laminar Region between Liquid and Packing Material Surface in the Packed Bed," J. Chem. Eng. Japan, 5, 132 (1972).

26. Greenkorn, R. A., and D. P. Kessler, "Dispersion in Heterogeneous Nonuniform Anisotropic Porous Media," Ind. Eng. Chem., 61, no. 9, 14 (1969). 
27. Dullien, F. A. L., "New Network Permeability Mode1 of Porous Media," AIChE J., 21, 299 (1975).

28. Petersen, E. E., "Diffusion in a Pore of Varying Cross Section," AIChE J., 4 , 343 (1958).

29. Payatakes, A. C., C. Tien, and R. M. Turian, "A New Model for Granular Porous Media: Part 1. Model Formulation," AIChE J., 19, $58(1973)$.

30. - "Part 2. Numerical Solution of Steady State Incompressible Newtonian Flow Through Periodically Constricted Tubes," AIChE J. 19,67 (1973).

31. Payatakes, A. C., and M. A. Neira, "Model of the Constricted "Type for Isotropic Granular Porous Media," AIChE J., 23, 922 (1977).

32. Happe1, J., "Viscous Flow in Multiparticle Systems: Slow Motion of Fluids Relative to Beds of Spherical Particles," AIChE J., 4, 197 (1958).

33. Newman, J., and W. Tiedemann, "Porous-Electrode Theory with Battery Applications," AIChE J., 21, 25 (1975).

34. Newman, J., and W. Tiedemann, "Flow-Through Porous Electrodes," in Adv. Electrochem. and Electrochem. Eng., Vo1. 11, John Wiley and Sons, Inc., New York, 1978.

35. Trainham, J., and J. Newman, "A Flow-Through Porous Electrode Mode1: Application to Metal-Ion Removal from Dilute Waste Streams," J. Electrochem. Soc., 124, 1528 (1977). 
36. Alkire, R., and R. Gould; "Analysis of Multiple Reaction Sequences in Flow-Through Porous Electrodes," J. Electrochem. Soc., $123,1842(1976)$.

37. Alkire, R., and B. Gracon, "Flow-Through Porous Electrodes," J. Electrochem. Soc., 122, 1594 (1975).

38. Wehner, J. F., and R. H. Wilhelm, "Boundary Conditions of Flow Reactor," Chem. Eng. Sci., 6, 89, (1956).

39. Trainham, J. A., and J. Newman, "The Effect of Electrode Placement and Finite Matrix Conductivity on the Performance of Flow-Through Electrodes," J. Electrochem. Soc., 125, 58 (1978).

40. Bennion, D., and J. Newman, "Electrochemical Removal of Copper Ions from Very Dilute Solutions," J. App1. Electrochem., 2, 113, (1972).

41. Sankarasubramanian, R., and W. N. Gi11, "Unsteady Convective Diffusion with Interphase Mass Transfer," Proc. ‥ Soc. Lond. A, 333,115 (1973).

42. Taylor, G., "Dispersion of Soluble Matter in Solvent Flowing S1owly Through a Tube," Proc. Roy. Soc. Lond. A, 219, 186 (1953).

43. Sherwood, T. K., Pigford, R. L., and C. R. Wilke, Mass Transfer, McGraw-Hi11 Book Company, Inc., New York, 1975.

44. Gunn , D. J., "Theory of Axial and Radial Dispersion in Packed Beds," Trans. Inst. Chem. Engrs., 47, T351, (1969).

45. Miyauchi, T., and T. Kirkuchi, "Axial Dispersion in Packed Beds," Chem. Eng. Sci., 30, 343 (1975).

46. Appe1, P. W., and J. Newman, "Application of the Limiting-Current Method to Mass Transfer in Packed Beds at Very Low Reynolds Numbers," AIChE J., 22, 979 (1976). 
47. Bar-Ilan, M., and W. Resnick, "Gas Phase Mass Transfer in Fixed Beds at Low Reynolds Numbers," Ind. Eng. Chem., 49, 313 (1957).

48. Hsuing, T. H., and G. Thodos, "Mass Transfer Factors from Actual Driving Forces for the Flow of Gases Through Packed Beds," Int. J. Heat Mass Trans., 20, 331 (1977).

49. Kato, K., Kubota, H., and C. Y. Wen, "Mass Transfer in Fixed and Fluidized Beds," Chem. Eng. Prog. Symp. Ser., 66, 87 (1970).

50. Kumar, S., Upadhyay, S. N., and V. K. Mathur, "Low Reynolds Number Mass Transfer in Packed Beds of Cylindrical Particles," Ind: Eng. Chem. Process Des. Dev., 16, 1 (1977).

51. Williamson, J., Bazaire, K. E., and C. J. Geankopolis, "Liquid Phase Mass Transfer at Low Reynolds Numbers," Ind. Eng. Chem. Fund. , 2, 126 (1963).

52. Wilson, E. J., and C. J. Geankopolis, "Liquid Mass Transfer at Very Low Reynolds Numbers in Packed Beds," Ind. Eng. Chem. Fund., 5, $9(1966)$.

53. Upadhyay, S. N., and G. Tripathi, "Liquid Phase Mass Transfer in Fixed and Fluidized Beds of Large Particles," I. Chem. Eng. Data, $\underline{20}, 20(1975)$.

54. Michaels, A. S., "Diffusion in a Pore of Irregular Cross Section A Simplified Treatment," AIChE J., 5, 270 (1959).

55. Houpeurt, A., "Sur 1'Écoulement des Gaz dans les Milieux Poreux," Rev. Inst. Fr. Petrole Ann. Combust. Liquides, 14, 1467 (1959). 
56. Batra, V. K., Fulford, G. D., and F. A. L. Dullien, "Laminar Flow Through Periodically Convergent-Divergent Tubes and Channels," Canadian J. Chem. Eng., 48, 622 (1970).

57. Dullien, F. L., and M. I. S. Azzam, "Flow Rate-Pressure Gradient Measurements in Periodica11y Non Uniform Capillary Tubes," AIChE J., 19, 222 (1973).

58. Sheffield, R. E., and A. B. Metzner, "Flow of Non Linear Fluids Through Porous Media," AIChE J., 22, 736 (1976).

59. Bird, R. B., Stewart, W. E., and E. N. Lightfoot, Transport Phenomena, John Wiley and Sons, Inc., New York, 1970.

60. Finlayson, B. A., The Method of Weighted Residuals and Variational Principles, Academic Press, New York, 1972.

61. Villadsen, J. V., Selected Approximation Methods for Chemical Engineering Problems, Danmarke Tekniske Hфjskole, Copenhagen, 1970.

62. Villadsen, J. V., and W. E. Stewart, "Solution of Boundary Value Problems by Orthogonal Collocation," Chem. Eng. Sci., 22, 1483 (1967).

63. Newman, J. S., Electrochemica1 Systems, Prentice-Ha11, Inc., Englewood Cliffs, N.J., Appendix C, 1973.

64. Newman, J., "The Fundamental Principles of Current Distribution and Mass Transport in Electrochemical Ce1ls," in Electroanalytical Chemistry, Marce1 Decker, Inc., N.Y., 6, 187 (1973).

65. Resnick, W., and R. R. White, "Mass Transfer in Systems of Gas and Fluidized Solids," Chem. Eng. Prog., 45, 377 (1949).

66. Eichorn, J., and R. R. White, "Partice1-to-Fluid Heat Transfer in Fixed and Fluidized Beds," Chem. Eng. Prog. Symp. Ser., 48, No. 4, 11 (1952). 
67. Dryden, C. E., Stang, D. A., and A. E. Withrow, "Mass Transfer in Packed. Beds at Low Reynolds Number," Chem. Eng. Prog., 49, 191 (1953).

68. Littman, H., Barile, R. G., and A. H. Pulsifer, "Gas-Particle Heat Transfer Coefficients in Packed Beds at Low Reynolds Numbers," Ind. Eng. Chem. Fund., 7, 554 (1968).

69. Petrovic, L. J., and G. T. Thodos, "Mass Transfer in the Flow of Gases Through Packed Beds," Ind. Eng. Chem. Fund., 7, 274 (1968).

70. Gliddon, B. J., and R. R. Cramefield, "Gas Particle Heat Transfer Coefficients in Packed Beds at Reynolds Numbers Between 2 and 100," Brit. Chem. Eng., 15, 489 (1970).

71. Karabelas, A. J., Wagner, T. H., and T. H. Hanratty, "Use of Asymptotic Relations to Correlate Mass Transfer Data in Packed Beds," Chem. Eng. Sci., 26, 1581 (1971).

72. Gunn, D. J., and J. F. C. De Souza, "Heat Transfer and Axial Dispersion in Packed Beds," Chem. Eng. Sci., 29, 1363 (1974).

73. Miyauchi, T., Matsumoto, K., and T. Yoshida, "Liquid Film Coefficient of Mass Transfer in Low Péclet Number Region for Sphere Packed Beds," I. Chem. Eng. Japan, $\underline{8}, 228$ (1975).

74. Miyauchi, T., Kataoka, H., and T. Kikuchi, "Gas Film Coefficient of Mass Transfer in Low Péclet Number Region for Sphere Packed Beds," Chem. Eng. Sci.,. 31, 9 (1976).

75. Miyauchi, T., Kikuchi, T., and K. H. Hsu, "Limiting Sherwood Number of Sphere Packed Beds by Electrical Method," Chem. Eng. Sci., 31, 493 (1976). 
76. Acrivos, A., and T. D. Taylor, "Heat and Mass Transfer from Single Spheres in Stokes Flow," Phys. Flulds, 5, 387 (1962).

77. Lea1, L. G., "On the Effective Conductivity of a Dilute Suspension of Spherical Drops in the Limit of Low Particle Péclet Number," Chem. Eng. Commun., 1, 21 (1973).

78. Levich, V. G., Physicochemical Hydrodynamics, Prentice-Ha11, New York, 1962.

79. Michelsen, M. L., and J. Villadsen; "The Graetz Problem with Axial Heat Conduction," Int. J. Heat Mass Transfer, 17, 1391 (1974) .

80. Miyauchi, T., Kikuchi, T., and H. Kataoka," Gas-Film Mass Transfer Coefficient in Packed Beds at Low Reynolds Numbers," Int. Chem. Eng., 12, 373 (1972).

81. Cornish, A. R., "Note on Minimum Possible Rate of Heat Transfer From a Sphere when Other Spheres are Adjacent to It," Trans. Instn. Chem. Eng., 43, T332 (1965).

82. Kunii, D., and M. Suzuki, "Particle-to-Fluid Heat and Mass Transfer in Packed Beds of Fine Particles," Int. I. Heat Mass Transfer, 10, 845 (1967).

83. Miyauchi, T., "Film Coefficients of Mass Transfer of Dilute Sphere-Packed Beds in Low Flow Rate Regime," J. Chem. Eng: Japan, 4, 238 (1971).

84. Lighthil1, M. J., "Contributions to the Theory of Heat-Transfer Through a Laminar Boundary Layer," Proc. Roya1 Soc. London A, 202, $359(1950$. 
85. Newman, J. E1ectrochemica1 Systems, Prentice-Ha11, Inc., Englewood C1iffs, N. J., p. 320 ff (1973).

86. Davis, H. M. and M. E. O'Neil, "The Development of Viscous Wakes in a Stokes Flow when a Particle is Near a Large Obstacle," Chem. Eng. Sci., 32, 899 (1977).

87. Moffatt, H. K., "Viscous and Resistive Eddies Near a Sharp Corner," J. Fluid Mech., 18, 1 (1964).

38. Ganatos, P., Pfeffer, R., and S. Weinbaum, "A Numerica1-Solution Technique for Three-Dimensional Stokes Flows, with Application to the Motion of Strongly Interacting Spheres in a Plane," J. Fluid Mech., 84, 79 (1978).

89. Chow, J., and K. Soda, "Heat and Mass Transfer in Laminar Flow in Conduits with Constriction," Trans. ASME J. Heat Transfer, August, 352 (1973).

90. Alkire, R., and P. K. Ng, "Studies of Flow-By Porous Electrodes Having Perpendicular Directions of Current and Electrolyte Flow," J. E1ectrochem. Soc., 124, 1220 (1977).

91. Gould R., private communication, July, 1978.

92. Coeuret, F., "L'Electrode Poreuse Percolante (EPP) - I. Transfert De Matiere En Lit Fixe," Electrochimica Acta, 21, 185 (1976).

93. Selman, J. R., Measurement and Interpretation of Limiting Currents, dissertation, University of California, Berkeley (1971).

94. Jo11s, K. R., and T. J. Hanratty, "Use of Electrochemical Techniques to Study Mass Trancfer Rates and Local Skin Friction to a Sphere in a Dumped Bed," AIChE J., 15, 199 (1969). 
95. Mandelbaum, J. A., and U. Böhm, "Mass Transfer in Packed Beds at Low Reynolds Numbers," Chem. Eng. Sc1., 28, 569 (1973).

96. Yip, H. H., Mass Transfer Coefficient in Packed Beds at Low Reynolds Numbers, M. S. Thesis, University of California, Berkeley (1973).

97. Hsueh, L., Diffusion and Migration in Electrochemical Systems, dissertation, University of California, Berkeley (1968).

98. Arvia, A. J., Bazán, J. C., and J. S. W. Carrozza, "Electrochemical Study of the Diffusion of Cupric Ion in Aqueous and Aqueous-G1ycero1 Solutions Containing Sulfuric Acid," Electrochimica Acta, 11, 881 (1966).

99. Lowenheim, F. A., (ed.), Modern Electroplating, 3rd edition, John Wiley and Sons, Inc., New York, 1974.

100. Guidebook and Directory of Metal Finishing, Metals and Plastics Publications, Inc., Hackensack, N. J., 1978.

101. Harrar, J. E., and C. L. Pomernacki, "Linear and Nonlinear Systems Characteristics of Controlled-Potential Electrolysis Cells," Analy. Chem., 45, 57 (1973).

102. Schroeder, R. R., and I Shain, Chem. Instrum., 1, 233 (1969).

103. Smith, G. F., and F. R. Duke, "Cerate Oxidemetry Determination of Glycerol," Ind. Eng. Chem. Ana1. Ed., 13, 558 (1941).

104. Churchi11, S. W., and R. Usagi, "A General Expression for the Correlation of Rates of Transfer and Other Phenomena," AIChE J., 8, $1121(1972)$. 
105. Acrivos, A., "On the Solution of the Convection Equation in Laminar Boundary Layer Flows," Chem. Eng. Sci., 17, 457 (1962).

106. Schlünder, E. U., "On the Mechanism of Mass Transfer in Heterogeneous Systems - In Particular in Fixed Beds, Fluidized Beds and In Bubble Trays," Chem. Eng. Sci., 32, 845 (1977).

107. Martin, H., "Low Péclet Number Particle-to-Fluid Heat and Mass Transfer in Packed Beds," Chem. Eng. Sci., 33, 913 (1978).

108. Newman, J., "Numerical Solution of Coupled, Ordinary Differential Equations," Ind. Eng. Chem. Fund., 7, 514 (1968). 
This report was done with support from the Department of Energy. Any conclusions or opinions expressed in this report represent solely those of the author(s) and not necessarily those of The Regents of the University of California, the Lawrence Berkeley Laboratory or the Department of Energy. 
TECHNICAL INFORMATION DEPARTMENT LAWRENCE BERKELEY LABORATORY

UNIVERSITY OF CALIFORNIA

BERKELEY, CALIFORNIA 94720 